\title{
3D Printed Bone-like Biopolymer Composites Inspired by Nacre
}

\author{
by \\ Mima Kurian
}

\author{
A thesis \\ submitted to Victoria University of Wellington \\ in fulfilment of the requirements for the degree of \\ Doctor of Philosophy in Chemistry
}

Victoria University of Wellington

2018 



\section{Declaration}

This thesis is a compilation of the work carried out at different research centres across New Zealand and Australia during 2014 - 2018. All the research, except mechanical testing and biological studies, has been conducted at Victoria University of Wellington, the rest were carried out externally in collaboration with various research labs. Uniaxial compression testing on 3D printed thermoplastic-based samples was carried out at Quest Integrity ltd, Wellington, by Mr Julian Broms. Uniaxial compression testing of all other samples was performed at the University of Otago, Christchurch, under the guidance of Dr Tim Woodfield and Dr Gabriella Brown; dynamic mechanical analysis was carried out at the University of Queensland, Brisbane, under the guidance of Dr Pratheep Kumar Annamalai and Dr Athanasia Septevani; nanoindentation testing was performed at the University of Auckland under the guidance of Dr Michelle Dickinson and Mr Thomas Loho. I was trained on all the instruments used, designed and performed the experiments by myself. Micro-CT analysis was performed by Mr Dane Gerneke (data collection), micro-CT technician at the Auckland bioengineering institute, University of Auckland. All biological experiments were designed, performed and analysed by Dr David Musson and Dr Tao Wang at the University of Auckland.

Mima Kurian 


\section{Abstract}

Bone tissue engineering and synthetic biomineralization are two widely researched areas, the principles of which have been combined from time to time in efforts to develop replacement materials for natural bone grafts. Nacre has been studied as a prospective bone graft material owing to its mechanical strength being comparable to that of natural bone. The extraordinary mechanical strength of nacre is attributed to its nanostructure. The McGrath research group developed a synthetic biomineralization method, herein called the McGrath method, that can be used to effectively replicate the elements of nacre's nanostructure in 2D biopolymer systems in laboratory conditions. Here, the applicability of the McGrath method in translating the calcium carbonate-based mineralization achieved in 2D films onto 3D printed chitosan hydrogel-based scaffolds is investigated. Thereby, enabling the fabrication of 3D chitosan-calcium carbonate composites with properties sought in the context of prospective load-bearing bone grafts.

In this work, considering the importance of interconnected porosity in an in vivo environment, nozzle extrusion-based 3D printing was employed to develop 3D structures with interconnected macropores, essentially imitating the porous structure of bone. The applicability of chitosan hydrogels as the printing ink in a custom-designed 3D printer was evaluated and quantified through rheological studies. The printing parameters and an appropriate experimental protocol were devised to fabricate stable 3D chitosan hydrogelbased scaffolds featuring physically crosslinked-layered structure with interconnected macropores. The effect of various drying techniques on retaining this porous structure in dried scaffolds and their swelling behaviour when soaked in a physiologically relevant solvent were explored using various techniques including cryo-scanning electron microscopy.

The strategies required to mineralize the as-fabricated 3D chitosan hydrogel-based scaffolds via the McGrath method, such that the mineralization achieved within the $3 \mathrm{D}$ scaffolds is similar to that obtained within 2D films, were elucidated. This included the use of polyacrylic acid (PAA), a crystal growth modifier. PAA has previously been shown to be important in achieving a pancake-like calcium carbonate formation, comprised of laterally growing nanoparticle aggregates which form in association with the organic matrix, in 2D films; such structures are observed in the early stages of nacre formation. By modulating the period of exposure of the $3 \mathrm{D}$ scaffolds to the mineralization solutions and the 
concentrations of these solutions, it was found that 3D composites with up to $40 \%$ calcium carbonate content and varying crystal morphology could be fabricated using this mineralization method. Importantly, it was observed that the calcium carbonate crystallites were intricately associated with the organic hydrogel matrix. This is another essential element observed in biomineral systems. Various techniques such as scanning electron microscopy (SEM), thermogravimetric analysis (TGA), energy dispersive spectroscopy (EDS) and X-ray diffraction (XRD) were employed for structural and compositional characterisation of the final composites.

The prospect of fabricating 3D chitosan-calcium carbonate composites via a single-step method using chitosan hydrogel preloaded with calcium carbonate crystallites as the printing ink in our custom-designed 3D printer was also investigated. This method was studied as a faster and less labour-intensive alternative to developing composites via the two-step method devised in this research whereby 3D hydrogel-based scaffolds are printed first and then mineralized via the McGrath method. The advantages and disadvantages of the two fabrication techniques are compared. The two-step fabrication method was found to be superior in terms of the properties explored and desired in the composite.

The behaviour of the chitosan hydrogel-based scaffolds and composites fabricated using the McGrath method, under different stress and strain regimes were also investigated. Mechanical tests performed on air-dried chitosan hydrogel-based scaffolds and composites showed that the compressive modulus, strength and indentation hardness values obtained were within the same order of magnitude as that of trabecular bone. Data from uniaxial compression tests showed that the yield, ultimate strength and compressive modulus of the 3D scaffolds vary with the total mineral content, morphology and size of the resultant crystallites in the composite. Composites with very low mineral content $\left(\sim 7 \% \mathrm{CaCO}_{3}\right.$ content) showed the best mechanical properties under uniaxial compressive stress (approximately $0.37 \mathrm{GPa}$ compressive modulus, $26 \mathrm{MPa}$ yield strength, and $31 \mathrm{MPa}$ ultimate strength). Nanoindentation tests showed that the nanoscale hardness and indentation modulus increased upon mineralization of the scaffolds but did not vary significantly as a function of the extent of mineralization. Dynamic mechanical analysis showed that the scaffolds (both mineralized and non-mineralized) can effectively dissipate stress without complete fracture when subjected to dynamic compressions within physiologically relevant loading frequencies $(1-15 \mathrm{~Hz})$ irrespective of the mineral content. The individual responses vary with loading frequency. 
Having ascertained the structural and mechanical attributes of the fabricated materials, their capacity to enable osteoblast cell attachment and proliferation was explored. Alamar Blue assay and confocal microscopy performed at various time points for samples exposed to in vitro cultured osteoblasts showed that chitosan hydrogel-based scaffolds and composites are biologically non-toxic and facilitate cell adhesion and proliferation. Furthermore, when osteoblasts were incubated with composites with low $\mathrm{CaCO}_{3}$ content, the number of cells increased significantly within 14 days.

The results of this research confirm that 3D printed chitosan hydrogel-based composites fabricated using the McGrath mineralization method featuring various structural and compositional imitations of bone and nacre shows considerable potential as future bone grafts materials. 


\section{Acknowledgement}

I came to New Zealand in the summer of 2014, thousands of miles away from my home, to pursue my passion for nanotechnology. The past three years have taught me many things, I travelled a lot and met a number of people, many of whom are an integral part of my life now. These wonderful experiences have played a huge role in the making of who I am today. I sincerely thank my supervisor Professor Kathryn McGrath (Kate) for the opportunity that paved way for this new chapter in my life and the MacDiarmid institute for providing the scholarship that funded my studies and living costs in New Zealand.

Kate, working with you has been truly enlightening. As your student, I have enjoyed a lot of freedom during my studies which, I believe has helped me grow as an independent researcher. I admire your patience, especially since you have had to read my thesis multiple times, and your excellent time management skills. I take this opportunity to express my heartfelt appreciation for your time, effort, knowledge and guidance throughout my studies at Victoria University. To my secondary supervisor Mr Ross Stevens, I have completely enjoyed your ideas and thoughts on how science and technology can be manipulated for the better or worse of the world. Thanks for supporting me throughout my study and for reviewing my thesis.

I take this opportunity to thank all the institutions, researchers and the technicians who have hosted me on multiple occasions and have been instrumental in the successful completion of my research. I would like to thank Mr Liam Gilbertson for custom-designing our 3D printer; Mr Julian Broms, mechanical technician, Quest Integrity ltd, for his valuable time in performing compression testing using Instron testing machines; Dr. Mark P. Staiger and Ms (probably Dr by now) Hina Ismail for hosting me at University of Canterbury and training me to use the dynamic mechanical analysis instrument; Prof Andrew Whittaker, Dr Pratheep Kumar Annamalai and Dr Athanasia Septevani for hosting me at the Australian institute of bioengineering and nanotechnology ( $\mathrm{AIBN})$ and training me to use the DMTA instrument; Dr Tim Woodfield and Dr Gabriella Brown for hosting me at the University of Otago and for providing expert advice on uniaxial compression testing and for training me to use the instrument, also huge thanks to Tim for reviewing Chapter 5 of this thesis; $\mathrm{Mr}$ Dane Gerneke, micro-CT technician at the Auckland bioengineering institute for performing Micro CT analysis; Dr David Musson, Dr Tao Wang and Prof Jillian Cornish for the carrying out the biological tests and providing expert advice for data analysis; Dr 
Michelle Dickinson and Mr Thomas Loho for hosting and training me to use the nanoindentation testing machine at Auckland University; Mr David Flynn, electron microscopy technician at Victoria University (VUW), for the training and support provided in using SEM, XRD and Cryo SEM; and Dr Mathew Crains, VUW, for training me to use the thermogravimetric analysis instrument; Dr Tim Brox, VUW for training me to use the rheology machine; and the school of ecology, VUW, for letting me use the laminar flow cabinet.

I truly cherish the beautiful friendship and support provided by the previous members of the McGrath research group. I thank Dr Saeedeh Afsar for the introductory lessons on biomineralization when I started my $\mathrm{PhD}$ research with the McGrath research group. Saeedeh Afsar, Davoud Zare and Lia Van Den Kerkhof, you guys have been (and will be) wonderful friends and I have totally enjoyed our chats, outings and dinners over the years. Special thanks to Lia for proofreading my thesis. Apart from them, I am also grateful for the wonderful company of my friends Sheen, Ellen Parth, Sreelakshmi, Aldritt, Shalini and Vaibhav, thank you all for making the last three years a memorable one. Special thanks to Parth for helping me in using the XRD instrument.

Above all, big thanks to my family for your love and support. To my parents, the values I uphold in my life, I have learned them from you and I am truly grateful to you for supporting me in living my dreams. To my dear sisters, thanks for all the stories that you have been telling me over the phone in the last 3 years, you made me feel like having been home all this time. To my dearly beloved husband Anil, thanks for being there for me at the times of sadness and happiness over the past two years. Half way though my $\mathrm{PhD}$ when life was monotonous, and I was missing a true companion, you came into my life like a gift from heaven. I am truly grateful for your companionship.

"I cried unto God with my voice- to God with my voice, and he gave ear unto me". 


\section{Table of Contents}

Declaration................................................................................

Abstract...................................................................................ii

Acknowledgement...................................................................vii

Table of Contents...........................................................................ix

List of Figures.................................................................... xiii

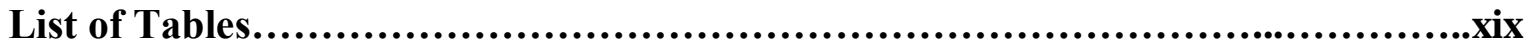

1 Introduction..........................................................................1

1.1 Motivation and inspiration....................................................1

1.2 Background overview........................................................

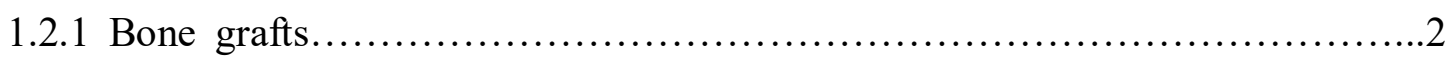

1.2.2a Bone: structure and composition....................................4

1.2.2b Biomineralization in bone...........................................6

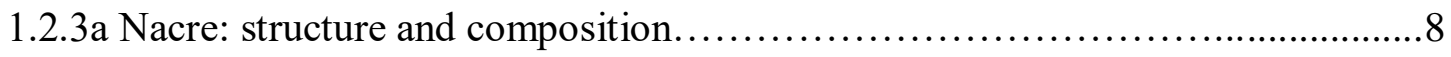

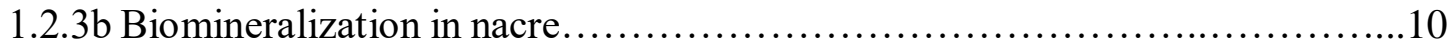

1.2.4 Biogenic calcium carbonate.........................................11

1.2.5 Chitosan hydrogels towards orthopaedic applications......................13

1.2.6 Matrix induced mineral growth: A strategy to improve mechanical properties...15

1.2.7 Porosity and its implications on the mechanical strength of engineered scaffolds............................................................... 16

1.2.8 Fabrication of porous 3D chitosan hydrogel-based scaffolds...................17

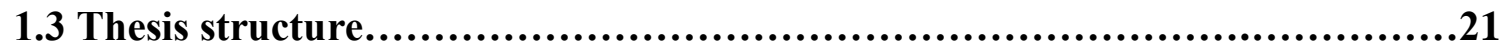

1.4 Bibliography.................................................................23

2 3D printed chitosan hydrogel-based scaffolds.................................31

2.1 Introduction.................................................................31

2.2 Materials and methods....................................................35

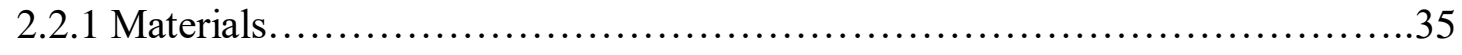

2.2.2 Rheological characterization....................................... 35 


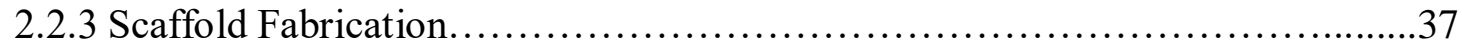

2.2.4 Scaffold Drying................................................. 42

2.2.5 Swelling behaviour of chitosan hydrogel-based scaffolds.....................42

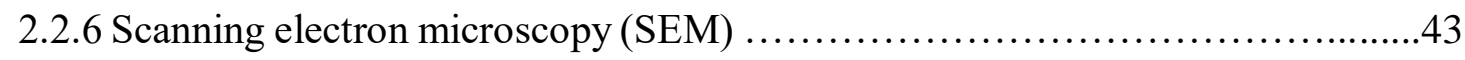

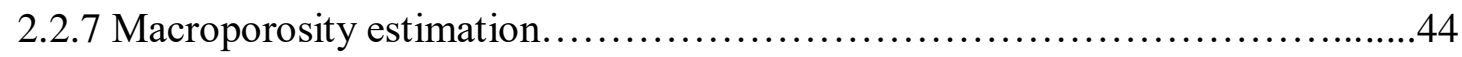

2.2.8 Micro-computed tomography (Micro-CT) analysis.........................44

2.3 Results and discussion...................................................46

2.3.1 Transition from 2D chitosan hydrogel-based films to 3D scaffolds............46

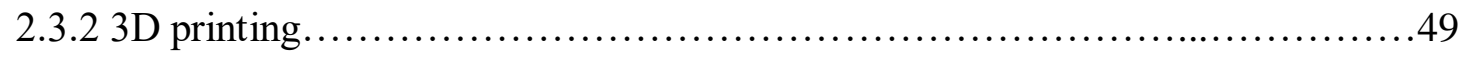

2.3.3 Effect of drying conditions on scaffold characteristics........................51

2.3.4 Swelling behavior of dried hydrogel-based scaffolds.......................54

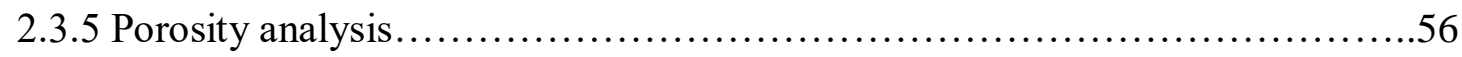

2.4 Conclusion................................................................60

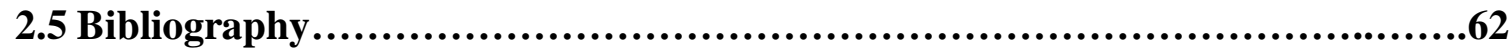

3 Mineralization of chitosan hydrogel-based scaffolds...........................67

3.1 Introduction.....................................................................67

3.2 Materials and methods.....................................................70

3.2.1 Materials........................................................ 70

3.2.2 Mineralization........................................................ 70

3.2.3 Scanning electron microscopy and energy dispersive spectroscopy..............71

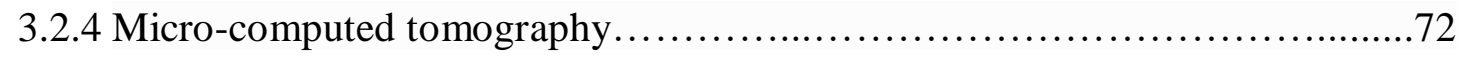

3.2.5 X-Ray diffraction spectroscopy ....................................... 72

3.2.6 Thermogravimetric analysis....................................... 72

3.3 Results and discussion...................................................73

3.3.1 Translating the mineralization achieved in 2D film to 3D scaffolds.............73

3.3.2 Mineralization of 3D chitosan hydrogel-based scaffolds.......................76

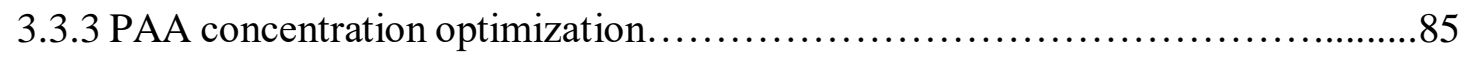

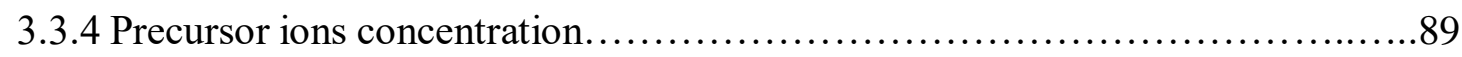

3.3.5 Period of exposure to mineralization media................................ 90

3.3.5a $0.5 \mathrm{M}$ precursor ion solution................................... 91

3.3.5b $0.25 \mathrm{M}$ precursor ion solution................................. 93

3.3.5c $0.1 \mathrm{M}$ precursor ion solution......................................94

3.3.6 Extent of mineralization............................................. 96 
3.3.7 Effect of printed macropores on the extent of mineralization. 101

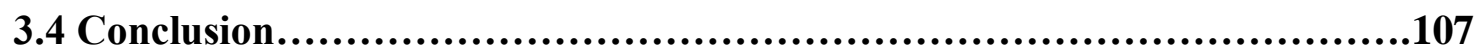

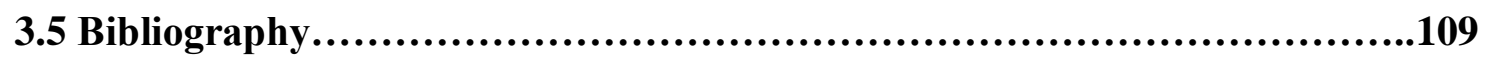

4 3D printed chitosan-calcium carbonate gel-based composite scaffolds ............115

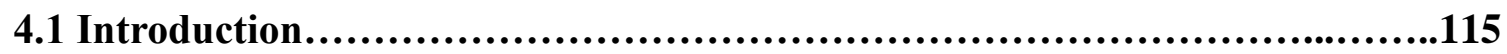

4.2 Materials and methods...................................................118

4.2.1 Chitosan-calcium carbonate hydrogel preparation.........................118

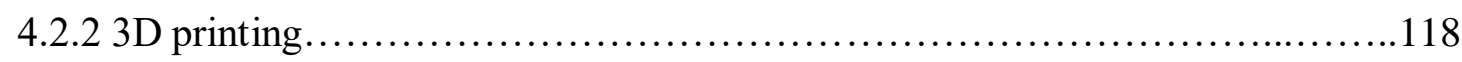

4.3 Results and discussion........................................................120

4.3.1 Rheology of $\mathrm{Ch}-\mathrm{CaCO}_{3}$ gels composite hydrogels....................... 120

4.3.2 3D printing using $\mathrm{Ch}-\mathrm{CaCO}_{3}$ blended hydrogel.........................124

4.3.3 Morphology of calcium carbonate crystallites in $\mathrm{Ch}-\mathrm{CaCO}_{3}$ composites........124

4.3.3a Blended hydrogel ink preparation...............................127

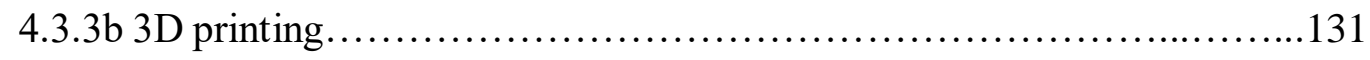

4.3.4 Effect of introduction of crystal growth modifier (PAA) ..................... 134

4.3.5 Mineral leaching in 3D printed $\mathrm{Ch}-\mathrm{CaCO}_{3}$ composites....................137

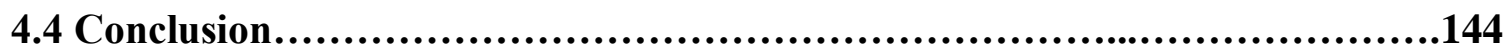

4.5 Bibliography..............................................................148

5 Mechanical properties of chitosan hydrogel-based scaffolds......................151

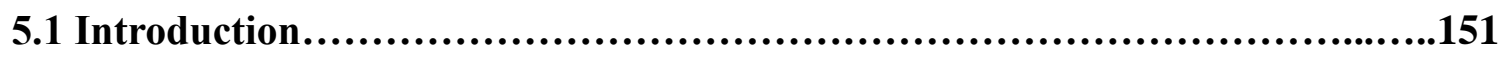

5.2 Materials and methods...................................................157

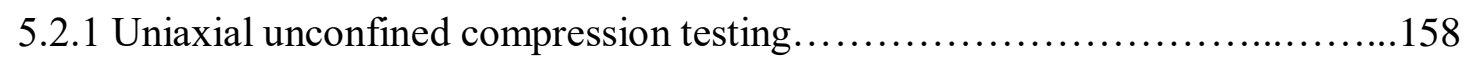

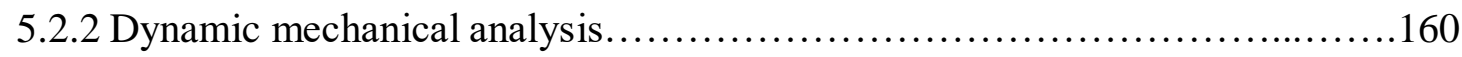

5.2.3 Hardness testing: nanoindentation.................................... 161

5.2.4 Statistical analysis................................................. 163

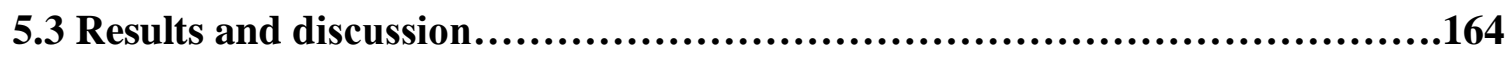

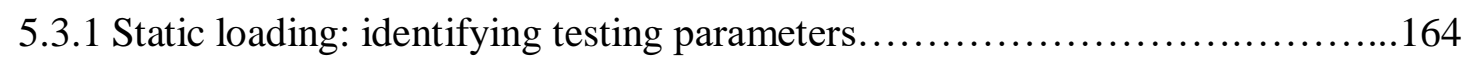

5.3 .1 a Effect of porosity.......................................... 167

5.3.1b Effect of introducing minerals in chitosan hydrogel-based scaffold.....169

5.3.1c Effect of the morphology of $\mathrm{CaCO}_{3}$ crystallites within chitosan- $\mathrm{CaCO}_{3}$ composites................................................. 174 
5.3.2 Dynamic loading.

5.3.3 Indentation hardness...................................................

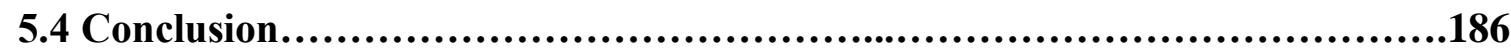

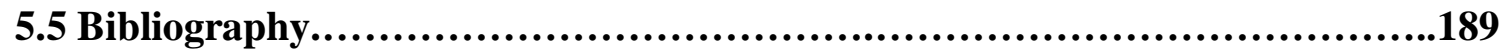

6 Biological performance of 3D printed chitosan hydrogel-based composites.......195

6.1 Introduction..................................................................195

6.2 Materials and methods.........................................................198

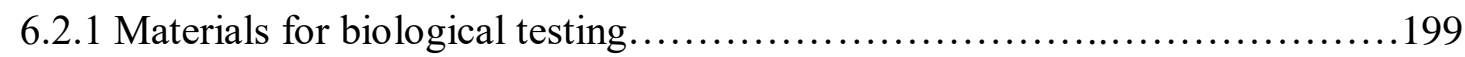

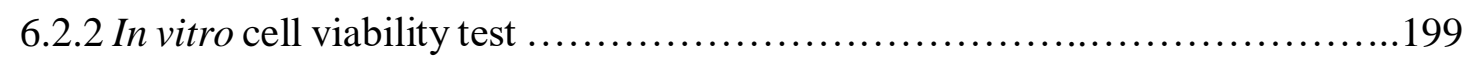

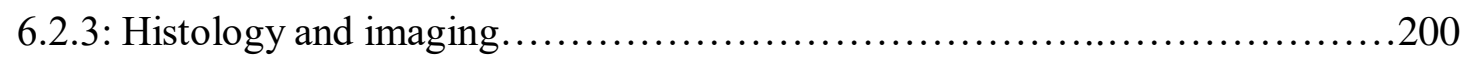

6.2.4: Statistical analysis..................................................201

6.3 Results and discussions......................................................202

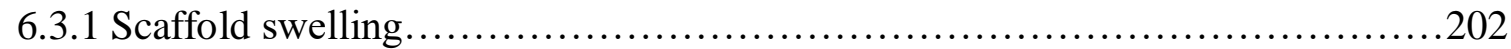

6.3.2 Cell viability, proliferation and adhesion ...................................204

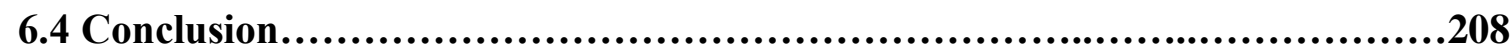

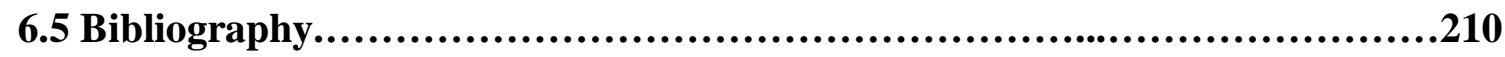

7 Conclusion \& Future Research....................................................213

7.1 Bibliography..............................................................220

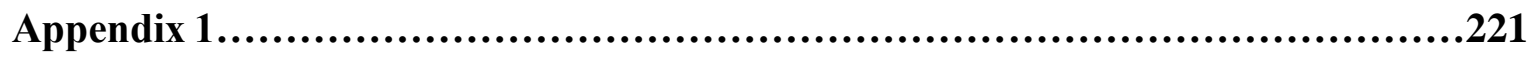




\section{List of Figures}

1.1 Hierarchal organization in bone.............................................

1.2 A representative image of the organization of collagen fibrils and HAP crystallites within bone at nanoscale

1.3 Hierarchal organization within the nacreous layer in red abalone (gastropods).........9

1.4 A schematic representation of the formation of nacreous layer in gastropods in a "Christmas tree" pattern via the formation of mineral bridges. Arrows A and B shows the organic interlayer imaged by SEM and the lateral boundary of tiles.

1.5 The crystallographic unit cell of calcite and SEM images of biogenic calcium carbonate of different morphologie.

2.1 The custom-designed 3D printer used to fabricate all 3D chitosan hydrogel basedscaffolds in this research.

2.2 (a) The specifications of the CAD model (magnified image) input for the 3D printer and (b) a schematic representation of the experimental procedure.

2.3 Screen shots of print parameters as configured in the slic3er software defining the speed of printing, layer height and infill pattern and parameters

2.4 Log-log plots of the variation of viscosity of chitosan hydrogels used for fabricating $2 \mathrm{D}(2 \% \mathrm{w} / \mathrm{v}$ chitosan in $1 \% \mathrm{v} / \mathrm{v}$ acetic acid solution) and 3D (5\% w/v chitosan in $2 \%$ v/v acetic acid solution) chitosan hydrogel-based scaffolds, with increasing shear rate.

2.5 Log-log plots of (a) strain sweep, $0.01 \%-500 \%$ at $1 \mathrm{~Hz}$ and; (b) frequency sweep $0.01 \mathrm{~Hz}$ to $100 \mathrm{~Hz}$ at $2 \%$ strain for freshly prepared and 6 days old $5 \%$ w/v chitosan hydrogel prepared in the presence of $2 \% \mathrm{v} / \mathrm{v}$ acetic acid.

2.6 (a) Optical images of chitosan hydrogel-based scaffolds in the wet state, left-hand side, and on the right is the corresponding cryo SEM image and (b) optical images of chitosan hydrogel-based scaffolds after air-drying, left-hand side, and on the right is the corresponding cryo-SEM image 
2.7 On the left are optical images of 3D chitosan hydrogel-based scaffolds in the dried state dried using one of the two drying techniques and the corresponding SEM images are shown on the right. (a) Critical point drying and (b) freeze drying.

2.8 Cryo-SEM images of air-dried chitosan hydrogel-based scaffolds (a) before and (b) after soaking in $1 \mathrm{X}$ PBS for 1 week.

2.9 A flowchart of the steps performed to calculate the total porosity of the 3D hydrogel scaffolds using the Skyscan CT analysis software.... .57

2.10 3D visualization of the open pores and closed pores inside the ROI selected within the reconstructed micro-CT image of a 3D chitosan hydrogel-based scaffold with $2 \mathrm{~mm}$ $\times 2 \mathrm{~mm}$ macropores. .58

3.1 SEM image of calcium carbonate crystallites developed on 2D chitosan hydrogelbased template via the McGrath mineralization method in the presence of polyacrylic acid on the left and that of nacre in its early stages of development. .68

3.2 Top row: optical images of 3D printed chitosan hydrogel-based scaffolds at various stages before and after mineralization according to the processing method used for $2 \mathrm{D}$ chitosan hydrogel-based templates. Bottom row: EDS images of the cross sections (in the $\mathrm{Z}$ direction marked by white arrow) of the final composites prepared via the processing method used for (a) 2D films and (b) the modified protocol developed in this work.

3.3 Optical images of chitosan hydrogel-based scaffolds mineralized via the McGrath method before (top left) and after (top right) air-drying. The SEM images of the airdried mineralized scaffolds showing the mineral growth observed (a) on the macropore walls; and (b) the external cylindrical region of the scaffold which was in contact with the media during the mineralization process......................77

3.4 SEM images of a 3D chitosan hydrogel-based scaffold mineralized via the McGrath method in the absence of crystal growth modifiers. .78

3.5 SEM images of 3D chitosan hydrogel-based scaffolds mineralized via the McGrath method in the presence of PAA

3.6 Schematic representation of the mineralization achieved on a 2D chitosan hydrogelbased template in the presence of PAA 
3.7 3D chitosan hydrogel-based scaffold mineralized via the McGrath method in the presence of PAA showing (a) an SEM image of a printed layer of the scaffold exposed directly to the mineralization media and (b) its corresponding EDS map.

3.8 SEM images of the top surface and lateral view of pancake-like calcium carbonate crystallites formed in the 3D chitosan hydrogel matrix when mineralized using the McGrath method in the presence of PAA.

3.9 A 3D visualization of the reconstructed micro-CT scans of chitosan hydrogel-based scaffold mineralized via the McGrath method in the presence PAA

3.10 SEM images of morphology of $\mathrm{CaCO}_{3}$ crystallites found in-between the printed layers (on the left-hand side) and on the regions exposed to the mineralization media (on the right-hand side) of 3D printed scaffolds mineralized via the McGrath method using $0.5 \mathrm{M}$ mineral precursor ion solutions $(1 \mathrm{~h}$ alternate soaking +1 week Kitano treatment) in the presence of (a) $0.5 \%$ (b) $1 \%$ (c) $1.5 \%$ (d) $2 \%$ (e) $2.5 \%$ (f) $3 \%$ (g)

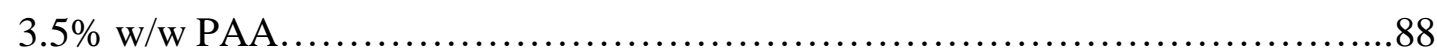

3.11 SEM images of $\mathrm{CaCO}_{3}$ crystallites formed in between the layers of the 3D scaffolds mineralized via the McGrath mineralization method ( $24 \mathrm{~h}$ alternative soaking +7 days Kitano) in the presence of $2.5 \%$ PAA using mineral precursor ion solutions of concentrations (a) $0.5 \mathrm{M}$; (b) $0.25 \mathrm{M}$; and (c) $0.1 \mathrm{M}$. Insets are higher magnification images of the crystallites showing the aggregation of nano-sized crystallites. .90

3.12 SEM images of calcium carbonate crystallites formed in between the layers of the 3D chitosan hydrogel-based scaffold as a result of alternately soaking in $0.5 \mathrm{M}$ mineral precursor ion (top row) solution for (a) $1 \mathrm{~h}$, (b) $2 \mathrm{~h}$ and (c) $24 \mathrm{~h}$; $0.25 \mathrm{M}$ mineral precursor ion solution (middle row) for (d) $1 \mathrm{~h}$ (inset is the higher magnification image of the same), (e) $2 \mathrm{~h}$ and (f) $24 \mathrm{~h}$; and $0.1 \mathrm{M}$ mineral precursor ion solution (bottom row) for (g) $1 \mathrm{~h}$, (h) $2 \mathrm{~h}$ and (i) $24 \mathrm{~h}$.

3.13 SEM images of the morphology of $\mathrm{CaCO}_{3}$ crystallites formed in between the layers of the 3D chitosan hydrogel-based scaffolds mineralized via the McGrath method by exposure to (a) $0.25 \mathrm{M}$ precursor ion solution alternately for $1 \mathrm{~h}$ each +3 days Kitano treatment; (b) $0.25 \mathrm{M}$ precursor ion solution alternately for $1 \mathrm{~h}$ each +4 days Kitano treatment; (c) only Kitano solution for 7 days; (d) $0.1 \mathrm{M}$ precursor ion solution alternately for $2 \mathrm{~h}$ each +3 days Kitano treatment; (e) $0.1 \mathrm{M}$ precursor ion solution 
alternately for $2 \mathrm{~h}$ each +4 days Kitano treatment (inset is a higher magnification image of the same) and (f) $0.1 \mathrm{M}$ precursor ion solution alternately for $2 \mathrm{~h}$ each +7 days Kitano treatment.

3.14 XRD plots for 3D chitosan hydrogel-based scaffolds mineralized via the McGrath method or only soaking in Kitano solution in the presence of PAA (2.5\% w/w w.r.t to dry wt. of chitosan)

3.15 A representative TGA plots for McGrath mineralized and non-mineralized chitosan hydrogel-based scaffolds

3.16 EDS image of chitosan hydrogel-based composites fabricated via $(\mathrm{a}, \mathrm{b}) 3 \mathrm{D}$ printing and $(\mathrm{c}, \mathrm{d})$ bulk gelation and mineralized via the McGrath mineralization method in the presence of $2.5 \%$ PAA.

3.17 XRD plots for 3D chitosan hydrogel-based scaffolds with or without macropores mineralized via the McGrath method (alternate soaking for $24 \mathrm{~h}$ in $0.5 \mathrm{M}$ precursor ion solutions + Kitano $(1$ week $))$ in the presence or absence of $2.5 \%$ w/w PAA with respect to the dry weight of chitosan.... 104

4.1 Log-log plot of the variation of viscosity as a function of the shear rate for a series of $5 \% \mathrm{w} / \mathrm{v}$ chitosan hydrogels prepared in the presence of $2 \% \mathrm{v} / \mathrm{v}$ acetic acid solution, with increasing percentage of added $\mathrm{CaCO}_{3}$

4.2 Log-log plot of the variation of viscosity as a function of the shear rate for a series of modified $\mathrm{Ch}-\mathrm{CaCO}_{3}$ blended hydrogels with varying percentage of added $\mathrm{CaCO}_{3}$ and acetic acid.

4.3 Log-log plot of the variation in the storage $\left(\mathrm{G}^{\prime}\right)$ and loss moduli $\left(\mathrm{G}^{\prime \prime}\right)$ as function of frequency $(\mathrm{Hz})$ for a series of the modified $\mathrm{Ch}-\mathrm{CaCO}_{3}$ hydrogels with varying percentage of added $\mathrm{CaCO}_{3}$ and acetic acid.

4.4 SEM images of crystallites obtained under different reaction conditions: (a-e) 10\%$50 \%$ calcium carbonate reacted with varying concentrations of acetic acid (using the same concentration for all reagents as those used to prepare the $\mathrm{Ch}-\mathrm{CaCO}_{3}$ gel-based printing inks with 5\% w/v chitosan but, in the absence of chitosan); and (f-j) Ch- 
$\mathrm{CaCO}_{3}$ hydrogels (used as the printing ink) with varying concentration of added $\mathrm{CaCO}_{3}(10 \%-50 \%)$. Also shown is $(\mathrm{k})$ the morphology of $\mathrm{CaCO}_{3}$ crystallites as supplied by the manufacturer.

4.5 Representative XRD data for (a) calcium carbonate as obtained from the manufacturer (b) the reaction product of $30 \%$ w/w $\mathrm{CaCO}_{3}$ (w.r.t $5 \%$ w/v chitosan) $+5 \%$ acetic acid (AA) i.e. the fake printing ink without chitosan; and (c) $\mathrm{Ch}-\mathrm{CaCO}_{3}$ hydrogel-based printing ink with $50 \%$ w/w $\mathrm{CaCO}_{3}$ (wr.t $5 \%$ w/w chitosan). Also shown are reference peaks for (d) calcium carbonate (rhombohedral calcite); and (e, f) calcium acetate hydrate of different unit cell structures (X'Pert Highscore 2.2c).... 130

4.6 SEM images of crystallites obtained under different reaction conditions: (a-e) the final $3 \mathrm{D}$ printed composites fabricated using $\mathrm{Ch}-\mathrm{CaCO}_{3}$ blended inks with varying concentration of $\mathrm{CaCO}_{3}$ added (10-50\%) after gelation in $1 \mathrm{M} \mathrm{NaOH}, \mathrm{pH}$ neutralization and air-drying; and (f-j) $10-50 \%$ calcium carbonate (w.r.t $5 \%$ w/v chitosan) reacted with varying concentrations of acetic acid and $1 \mathrm{M}$ ethanolic $\mathrm{NaOH}$

4.7 Representative XRD data (a) of the reaction products of $50 \%$ w/w $\mathrm{CaCO}_{3}$ (w.r.t $5 \%$ w/v chitosan) $+10 \% \mathrm{v} / \mathrm{v}$ acetic acid $+1 \mathrm{M} \mathrm{NaOH}$ (ethanolic) i.e. the fake composite without chitosan; and (b) the final 3D printed composite fabricated using $\mathrm{Ch}-\mathrm{CaCO}_{3}$ blended hydrogel loaded with $50 \% \mathrm{w} / \mathrm{w} \mathrm{CaCO}_{3}$. Also shown are reference peaks for (c) calcium carbonate (calcite); and (d, e) calcium acetate hydrate with different unit cell structures (X'Pert Highscore 2.2c).

4.8 A comparison of the $\mathrm{CaCO}_{3}$ morphologies formed in the $3 \mathrm{D}$ printed chitosan- $\mathrm{CaCO}_{3}$ composites fabricated via 3D printing using (a) $\mathrm{Ch}-\mathrm{CaCO}_{3}$ blended inks loaded with $30 \% \mathrm{w} / \mathrm{w} \mathrm{CaCO}_{3}$ and $2.5 \% \mathrm{w} / \mathrm{w}$ PAA and (b-c) chitosan hydrogel as the printing ink followed by mineralization of the as fabricated chitosan hydrogel-based scaffold via the McGrath mineralization method in the presence of $2.5 \%$ w/w PAA (w.r.t the dry weight of chitosan used). (d, e) are the corresponding optical images of the final composites based on (a) and (b) respectively

4.9 Thermal decomposition behaviour of blended inks with varying concentration of calcium carbonate added and the corresponding 3D printed composites. 141 
5.1 A representative stress-strain plot for chitosan- $\mathrm{CaCO}_{3}$ scaffolds under static compressive loading using a $5 \mathrm{kN}$ load cell after applying the preload correction in order to consider the strain induced due to the applied $2 \mathrm{~N}$ preload.

5.2 A typical load-displacement curve for a chitosan- $\mathrm{CaCO}_{3}$ composite scaffold for indentations performed in constant displacement mode.

5.3 Representative stress-strain plots for chitosan hydrogel-based scaffolds under compression. 166

5.4 Variation in the compressive modulus, yield and ultimate strength of air-dried chitosan hydrogel-based scaffolds fabricated with different macropore dimensions. 168

5.5 Variation in the compressive modulus, yield strength and ultimate strength of chitosan$\mathrm{CaCO}_{3}$ composites with different percentage calcium carbonate content fabricated via the McGrath method in comparison to non-mineralized chitosan hydrogel-based scaffolds.... 171

5.6 Variation in the dynamic storage, loss and complex moduli of chitosan hydrogel-based scaffolds with increasing $\mathrm{CaCO}_{3}$ content introduced via the McGrath mineralization method.

5.7 Variation in the compressive modulus and the yield strength of samples subjected to DMA-frequency scans in comparison to those of un-stressed samples.

5.8 Variation in the average combined hardness and indentation elastic modulus of a nonmineralized scaffold and composites with varying percentage mineral content......184

6.1 Representative optical images of the swelling observed in air-dried 3D chitosan hydrogel-based scaffolds and composites after incubation in the culture media....203

6.2 Variation in osteoblast cell viability (percentage) with the period of incubation and the mineral content of 3D printed chitosan hydrogel-based scaffold...................205

6.3 Confocal microscopy images of 3D chitosan hydrogel-based scaffolds and composites after 14 days of incubation with osteoblasts. .206

A1.1 Stress-strain plots for 3D printed ABS-based structures with different internal architectures 223 


\section{List of Tables}

1.1 Mechanical properties of bone and its microstructures ...........................6

2.1 Swelling behaviour of chitosan hydrogel-based scaffolds dried via different methods after soaking in 1X PBS for one week........................................... 55

2.2 The porosity of 3D printed chitosan hydrogel-based (dried) with two different macropore dimensions, estimated based on their respective CAD model and by using $2 \mathrm{D}$

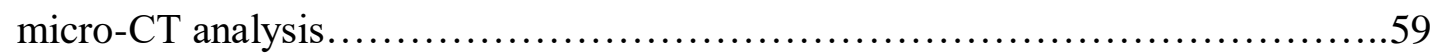

3.1 The $\mathrm{pH}$ of the different mineralization solutions used before and after addition of $2.5 \%$ w/w PAA w.r.t dry weight of chitosan used................................... 71

3.2 The variation in the diameter of a 3D printed chitosan hydrogel-based scaffold in the wet state prior to and during the various stages of the mineralization process as used for 2D films 75

3.3 A summary of the different morphologies of $\mathrm{CaCO}_{3}$ observed in SEM images of the chitosan- $\mathrm{CaCO}_{3}$ composite obtained by mineralization via the McGrath method using $0.5 \mathrm{M} \mathrm{Ca}^{2+}$ and $\mathrm{HCO}_{3}{ }^{-}$(1 $\mathrm{h}$ alternate soaking +1 week soaking in Kitano solution) and varying concentrations of PAA w.r.t to dry weight of chitosan

3.4 The percentage $\mathrm{CaCO}_{3}$ content within 3D chitosan hydrogel-based scaffolds mineralized via the McGrath method using increasing concentrations of mineral precursor ions or Kitano method in the presence of $2.5 \%$ w/w PAA w.r.t dry weight of chitosan used, as analysed via TGA and acid dissolution method.

3.5 The total porosity of the final air-dried chitosan- $\mathrm{CaCO}_{3}$ scaffolds fabricated by mineralization of chitosan hydrogel-based scaffolds with $1.5 \mathrm{~mm} \times 1.5 \mathrm{~mm}$ macropores (dimension in the wet state), via different mineralization methods compared to that of non-mineralized scaffolds.

4.1 A comparison between the actual and expected minerals content within the final 3D printed composite ( $15 \mathrm{~mm}$ diameter $\times 4 \mathrm{~mm}$ high) fabricated using $\mathrm{Ch}-\mathrm{CaCO}_{3}$ blended inks with different calcium carbonate loading percentage. 
5.1 A representative suite of measurements obtained for one sample of each type......168

5.2 The percentage residual volume obtained via TGA analysis of the composites after compression testing ................................................... 170

5.3 Variation in the compressive modulus, yield and the ultimate strengths of chitosan$\mathrm{CaCO}_{3}$ composites fabricated via McGrath mineralization of chitosan hydrogel-based scaffolds by alternately soaking in $0.1 \mathrm{M}$ or $0.25 \mathrm{M}$ mineral precursor ions solution and soaking in Kitano solution, for varying time periods...................... 175

5.4 Hardness values for different regions within a sample......................... 183

A1.1 The variation in the compressive strength of the ABS-based 3D printed structures with different architectures. .223 


\section{Introduction}

\subsection{Motivation and inspiration}

Bone and nacre are two different yet similar biocomposites, when many of their attributes are considered and compared. Bone is a protein-based nanocomposite of collagen and hydroxyapatite, whereas nacre is a carbohydrate-based nanocomposite of calcium carbonate with the organic matrix mostly comprised of chitin. Their differences include, for example, their composition, architecture and ability to support biological cell growth while having similar mechanical characteristics [1]. Nacre's characteristics including strength and fracture resistance, biocompatibility, non-toxicity and non-inflammatory properties are highly advantageous from a biomedical perspective which make it suitable for use as bone grafts (see reference [2] for use of nacre as bone grafts in sheep).

In both nacre and bone, the exceptional mechanical strength is mostly attributed to their respective composition and nanostructure, thus directing research towards the replication of these nanostructures in synthetic materials. Of the two materials, nacre is structurally and chemically simpler. Developing a nacre-like material is therefore preferentially more straightforward than replicating bone while having the desirable mechanical responses and therefore having the potential to be used a bone graft material. The nanostructure of nacre has been replicated in laboratory via different methods [3-6].

The McGrath research group has been studying the biomineralization in molluscs in order to develop synthetic biomineralization strategies to replicate the nanostructure in nacre on a laboratory scale [7-11]. The mineralization technique thus developed can be tailored and used to mineralize both carbohydrate and protein-based 2D hydrogel films. In this research, this mineralization technique is tailored to obtain calcium carbonate mineralization in a $3 \mathrm{D}$ carbohydrate-based scaffold, in an attempt to develop 3D composite structures that have properties comparable to bone and nacre.

Broadly, this thesis investigates whether a hierarchically porous 3D biopolymer-based composite material with nacre-inspired nanostructure has mechanical strength and biocompatibility attributes comparable to natural bone. This research is directed towards 
development of materials to be used as bone grafts that are cost effective, biocompatible and have the necessary mechanical attributes to support load bearing applications.

An ideal bone graft must be biocompatible; non-toxic; osteoconductive; osteoinductive; cost effective; invoke no inflammatory response in vivo; and have an optimal balance between its porosity to support cell mobility and mechanical compatibility to the implant site [12]. Replicating the complex nanostructure of bone to develop artificial bone grafts with similar properties is extremely difficult. Herein, 3D biopolymer-based composites with organicinorganic components similar to that of nacre and macropores incorporated into the structure to facilitate vascularisation inspired by the porous architecture of bone; were fabricated in an attempt to develop porous high strength composites for potential use as artificial bone grafts.

To summarise, nanocomposites are fabricated with architectural features and composition over a range of length scales that are inspired from both bone and nacre. Their mechanical and biological compatibility is then studied in an effort to develop future bone graft materials.

\subsection{Background overview}

\subsubsection{Bone grafts}

Bone is the second most transplanted tissue in the human body after blood, with over two million procedures performed as part of oncological surgeries, traumatology, revision prosthetic surgery and spine surgeries performed every year worldwide [13, 14]. Damage to bone tissues can significantly affect a patient's quality of life, from an inability to perform basic tasks like walking to psychological wellbeing, as even in the case of the most minor damage, the healing process is spontaneous but slow. When such defects are large enough they may not regenerate via normal physiological processes and may require intervention in the form of bone grafts to assist the healing process. A bone substitute/graft can be defined as "a synthetic, inorganic or biologically organic combination which can be inserted for the treatment of a bone defect instead of autogenous or allogenous bone" [15]. Bone grafts may be cortical, cancellous, or corticocancellous, depending on whether structural strength or osteogenesis or both is required respectively. An ideal bone graft would be corticocancellous, providing both functions [16]. 
Current clinically proven cancellous or cortical bone grafts are autologous, allogeneic or xenogenic bone tissues. Autologous transplants, where the tissue is harvested from the patient themselves, are considered the best, as these possess the essential components to achieve osteoconduction (bone formation at implant surface), osteoinduction, osteogenesis (bone formation), and immunogenic compatibility. However disadvantages, such as the surgery being expensive, patients requiring a second surgery to harvest the transplant tissue and complications such as bone non-unions and blood loss limits the use of autografts [17, 18]. Similarly, allografts which are bones taken from cadavers have side effects such as the chances of transmitting diseases and causing severe immune responses. Xenografts, which are typically high temperature treated bovine bone, ensures higher osteointegration of the grafts and lowers the incidences of disease transmittance but, the treatment period is rather long (12-18 months) and painful [18, 19]. These persistent side effects encourage new therapeutic strategies such as the development of artificial/synthetically engineered bone grafts that mimic the natural bone microarchitecture, mechanical strength and bioactivity hence replacing the need to use natural grafts.

Ceramic bone grafts that are calcium-based substitutes such as hydroxyapatite (HAP), tricalcium phosphate (TCP), calcium phosphate cement and calcium sulfate have, for more than 50 years, been used instead of natural bone grafts [15, 20, 21]. Such substitutes are deemed biocompatible, osteoconductive and bioresorbable as the graft material is slowly resorbed into the body as new bone formation occurs. Most of these ceramics-based substitutes are clinically approved and have been used in humans for non-load bearing applications such as dental and craniofacial applications without adverse side effects. However, these are unsuitable for load bearing applications as ceramics are usually brittle and their highly porous structure results in a maximum compressive strength of up to 60 $\mathrm{MPa}$ (combination of HAP and TCP) which is much lower than that of cortical bone (140$200 \mathrm{MPa})$ [13].

Polymer and polymer-composites as standalone devices or as extenders on auto/allo grafts were introduced more recently [22]. Composites based on biodegradable synthetic polymers such polylactic acid or polyglycolic acid depict better osteoconductivity, osteoblast differentiation and osteogenesis compared to monolithic structures such as HAP [23, 24]. Addition of polymers improve the mechanical strength and fracture toughness of ceramics and the mechanical stability of the biopolymers [25]. In terms of the fabrication techniques used to develop biopolymer-ceramic composites, biomimetic routes have been found to be 
more advantageous in terms of developing composites with higher mechanical strength in comparison to those fabricated via direct blending of the components and casting to develop $3 \mathrm{D}$ structures. [26, 27].

Developing biomimetic techniques to effectively replicate the properties of biocomposites existing in nature is an extremely difficult task considering the level of structural complexity involved. Nature is adept in controlling mineralization and manipulating the compositional organization within living organisms to the smallest measurable order, resulting in stable artefacts with properties that are not achievable under laboratory conditions. Natural nanocomposites such as bone, teeth (dentine and enamel), nacre and sea urchin spines are beautiful examples of this. The intimate association between the organic matrix and the inorganic minerals within them is the reason for their exceptional mechanical strength and unusual resistance to different types of external loads. Thus, it is necessary to gain in-depth knowledge of the individual components of these composites and the nature of the interaction between them before attempting to replicate them in the laboratory. The microstructure and mineralization occurring in bone and nacre are studied in detail in order to develop a composite inspired from their architecture and composition.

\subsection{2a Bone: structure and composition}

Bone is a natural biocomposite composed of approximately $60 \%$ mineral, $30 \%$ matrix and $10 \%$ water. It is also considered a living tissue with cellular content of about $15 \%$ of its weight [28]. The bone matrix is composed of highly aligned type I collagen fibrils but, overall the fibre orientation is anisotropic. This feature contributes predominantly to the tensile strength of bone. The mineral component is mostly calcium phosphates in the form of carbonated HAP $\left[\mathrm{Ca}_{10}\left(\mathrm{PO}_{4}\right)_{6}(\mathrm{OH})_{2}\right]$, which is nucleated within the collagen matrix and is closely associated with it. HAP exists as flat plate-like nanocrystals $50 \mathrm{~nm}$ long, $25 \mathrm{~nm}$ wide and 1.5 - $4 \mathrm{~nm}$ thick [29].

The immaculately complex structure of this organ can be subdivided into up to seven hierarchical levels of organization. Studying the hierarchical organization of bone is fundamental to understanding its mechanical characteristics, as it is this feature that combines the toughness of inorganic minerals and the flexibility of protein-based macromolecules to give the extraordinary strength of bone. Interestingly, bone undergoes continuous remodelling and growth with time under continued external (stress) as well as internal stimuli. It accommodates a network of living cells which are interconnected via the 
extensive pore channels within its microstructure. Bone cells or osteoblasts respond to external mechanical pressures and initiate localized mineralization proportional to the stress it is being subjected to; hence the microstructure and mineral content of bones from different regions of the skeletal system differ owing to the variation in the stress rate that each region is subjected to on a daily basis [30]. The hierarchy can be summarized in five levels (which can be expanded to seven levels) as shown in Figure 1.1 below [31]:

- the macrostructure: cancellous and cortical bone;

- the microstructure $(10-500 \mu \mathrm{m})$ : haversian systems, osteons, single trabeculae;

- the sub-microstructure $(1-10 \mu \mathrm{m})$ : lamellae;

- the sub-nanostructure (below a few hundred nanometers): molecular structure of constituent elements, such as mineral, collagen, and non-collagenous organic proteins; and

- the nanostructure (from a few hundred nanometers to $1 \mu \mathrm{m}$ ): fibrillar collagen and embedded mineral.

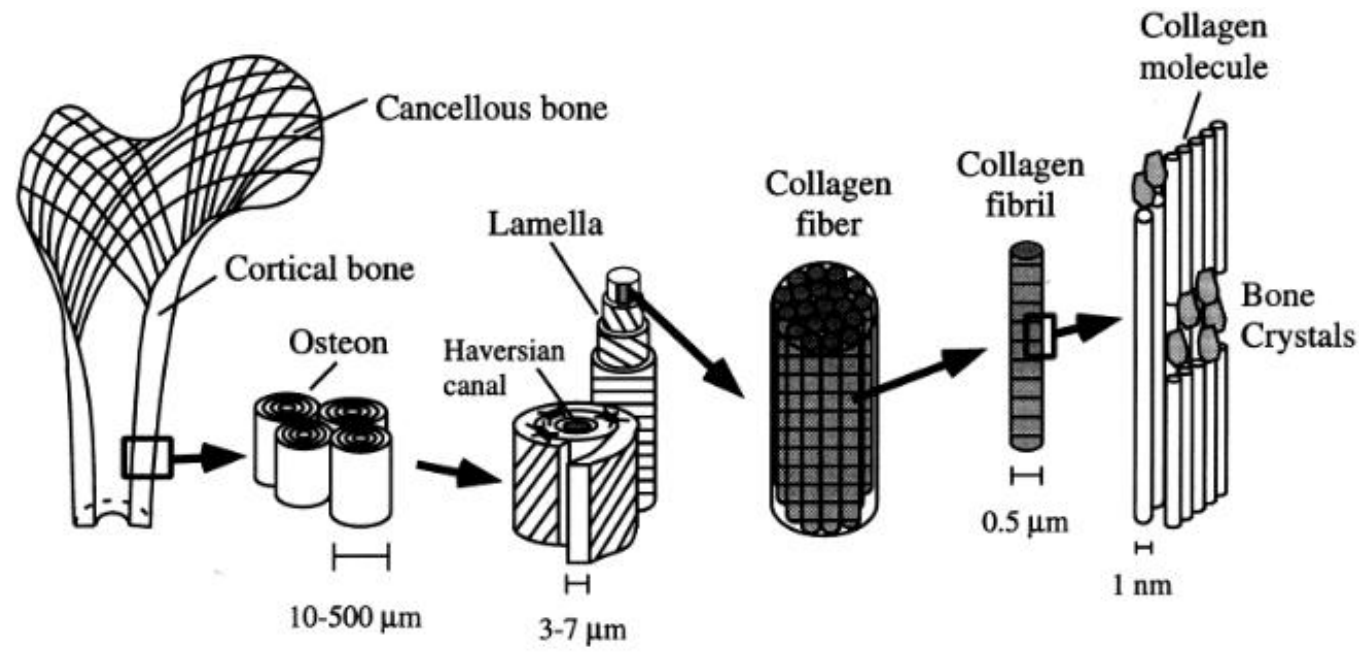

Microstructure

Nanostructure

- Macrostructure

Sub-microstructure

Sub-nanostructure

Figure 1.1: Hierarchal organization in bone reprinted with permission from [31].

Table 1.1 gives the mechanical properties of bone (cortical and cancellous/trabecular bone) and its microstructures. Owing to its organization, compact bone (5 - $10 \%$ porosity with 10 - $50 \mu \mathrm{m}$ pores) has a compressive strength in the range of 100 - $230 \mathrm{MPa}$ and a Young's modulus between 7 - $30 \mathrm{GPa}$. In contrast, the spongy trabecular bone, has a compressive strength of 2 - $12 \mathrm{MPa}$ and Young's modulus of 0.05 - 0.5 GPa [32], nevertheless, the values 
are exceptionally high given the highly porous structure of trabecular bone $(75-85 \%$ porosity with $300-600 \mu \mathrm{m}$ diameter pores) [33].

\begin{tabular}{lcc}
\hline Property & $\begin{array}{c}\text { Cortical } \\
\text { bone }\end{array}$ & $\begin{array}{c}\text { Cancellous } \\
\text { bone }\end{array}$ \\
\hline Compressive strength/MPa & $100-230$ & $2-12$ \\
Flexural, tensile strength/MPa & $50-150$ & $10-20$ \\
Strain to failure/\% & $1-3$ & $5-7$ \\
Fracture toughness/MPam ${ }^{1 / 2}$ & $2-12$ & - \\
Young's modulus/GPa & $7-30$ & $0.5-0.05$ \\
\hline & \multicolumn{2}{c}{ Young's modulus } \\
\hline Architecture & $14-20$ \\
Wet specimen (macrostructural) & \multicolumn{2}{c}{5.4} \\
Wet specimen (microstructural) & 22 \\
Dry specimen (submicrostructure) & 22 \\
\hline
\end{tabular}

Table 1.1: Mechanical properties of bone and its microstructures. Reprinted with permission from [32].

The basic unit of the hierarchal organisation in bone is the nanocomposite comprised of collagen fibrils and HAP platelets. Studying their interaction at the nanoscale is essential in understanding the initiation and evolution of calcium phosphate mineralization in bone. This can contribute to developing a better understanding of the influence of the organic and the mineral components in the final mechanical strength of bone and hence aid in replicating it in laboratory conditions.

\subsection{2b Biomineralization in bone}

In bone, collagen fibrils and apatite crystals are uniaxially oriented. Osteoblast cells secrete collagen molecules that then self-assemble to form fibrils. Individual fibrils assume a triple helical structure and are separated axially by a $40 \mathrm{~nm}$ gap between the $-\mathrm{NH}_{3}$ of the first fibril and the $-\mathrm{COOH}$ terminals of the next, the adjacent fibril is positioned in a staggered arrangement with a $67 \mathrm{~nm}$ gap as shown in Figure 1.2 [34]. 


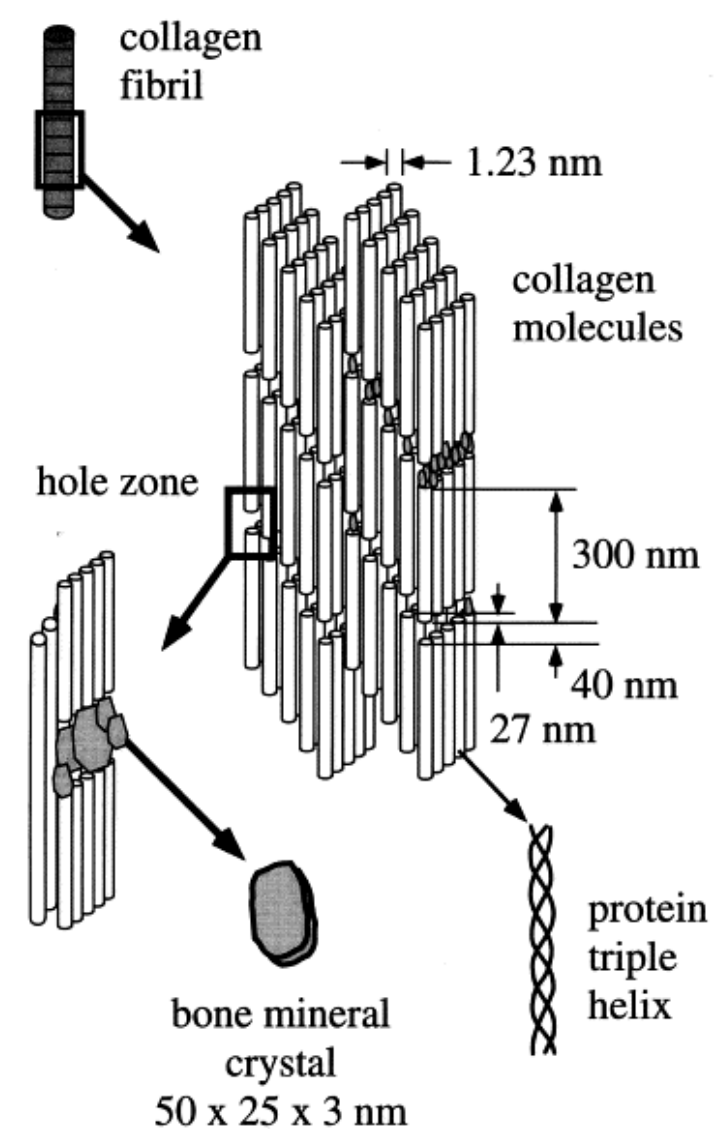

Figure 1.2: A representative image of the organization of collagen fibrils and HAP crystallites within bone at nanoscale, reprinted with permission from [31].

It was previously assumed that the collagen matrix is simply an inactive template for mineralization, and that non-collagenous proteins were responsible for the apatite nucleation in bone. Recently however it was determined that mineral nucleation is initiated in the gaps within the collagen matrix, owing to the high concentration of negative charge that results in the attraction of positively charged $\mathrm{Ca}^{2+}$ ions, hence suggesting that the nucleation process actively involves collagen fibrils as well (Figure 1.2) [35]. Studies have shown that negatively charged non-collagenous proteins interact with amorphous calcium phosphate (ACP) nucleates (formed at the very initial stages of $\mathrm{CaP}$ nucleation), leading to facilitated inflow of ACP into the collagen fibril at the positively charged $\mathrm{C}$ terminal of collagen. Further, the charged amino acids at the fibril gaps provide the nucleation sites for apatite nucleation from ACP. Hence, biomineralization in bone is the combined effect of collagenous as well as non-collagenous proteins. Non-collagenous proteins are also known to direct the crystal growth after nucleation and prevent extra fibrillary mineralization during the initial stages $[7,36]$. 
When under stress, it is observed that the load transfer mechanism at a nanoscale is facilitated via the electrostatic interaction between the inorganic and the organic phases. Also, stress and strain distribution occur via combined action of the energy dissipating capacity of collagen molecules through uncoiling of their triple helical structure and the stiffness of minerals growing in a direction parallel to individual fibrillary axes. The intrinsic association of this nanocomposite over the long range order is expected to amplify these nanoscale mechanical properties of bone [37].

\subsection{3a Nacre: structure and composition}

Nacre is an inorganic-organic nanocomposite with approximately 95\% mineral (calcium carbonate) and 5\% organic matrix with the matrix interlacing the minerals like mortar and brick respectively [38]. The primary structural component is pseudohexagonal aragonite tablets, a polymorph of calcium carbonate about $0.5 \mu \mathrm{m}$ thick and about $5-10 \mu \mathrm{m}$ in width [39]. The organic matrix is composed of $\beta$-chitin, silk-like proteins, and acidic glycoproteins rich in aspartic acid [40]. Nacre formation is a dynamic process that continues over the life time of the organism unless the influx of mineral ions stops. The structure of nacre which is generally described as the "brick-mortar" architecture can be explained in five levels of hierarchy from nano to macroscale based on the dimensions of the components involved as shown in Figure 1.3 [41]:

- Level I: molecular structure of the chitin fibers and atomic crystalline structure of the calcium carbonate in the form of aragonite.

- Level II: mineral bridges between aragonite tiles (diameter of $20-60 \mathrm{~nm}$ ) and random dispersion of chitin fibrils interlacing the tiles (thickness $\sim 20 \mathrm{~nm}$ ).

- Level III: hexagonal aragonite tiles (lateral dimensions of $10 \mu \mathrm{m}$ and thickness of $0.5 \mu \mathrm{m})$.

- Level IV: Mesolayers (0.1 - $0.3 \mathrm{~mm}$ thick) that are formed by combination of thick layers of organic matrix (thickness $\sim 200 \mu \mathrm{m}$ ) interlacing aragonite tiles.

- Level V: macroscopic nacre. 

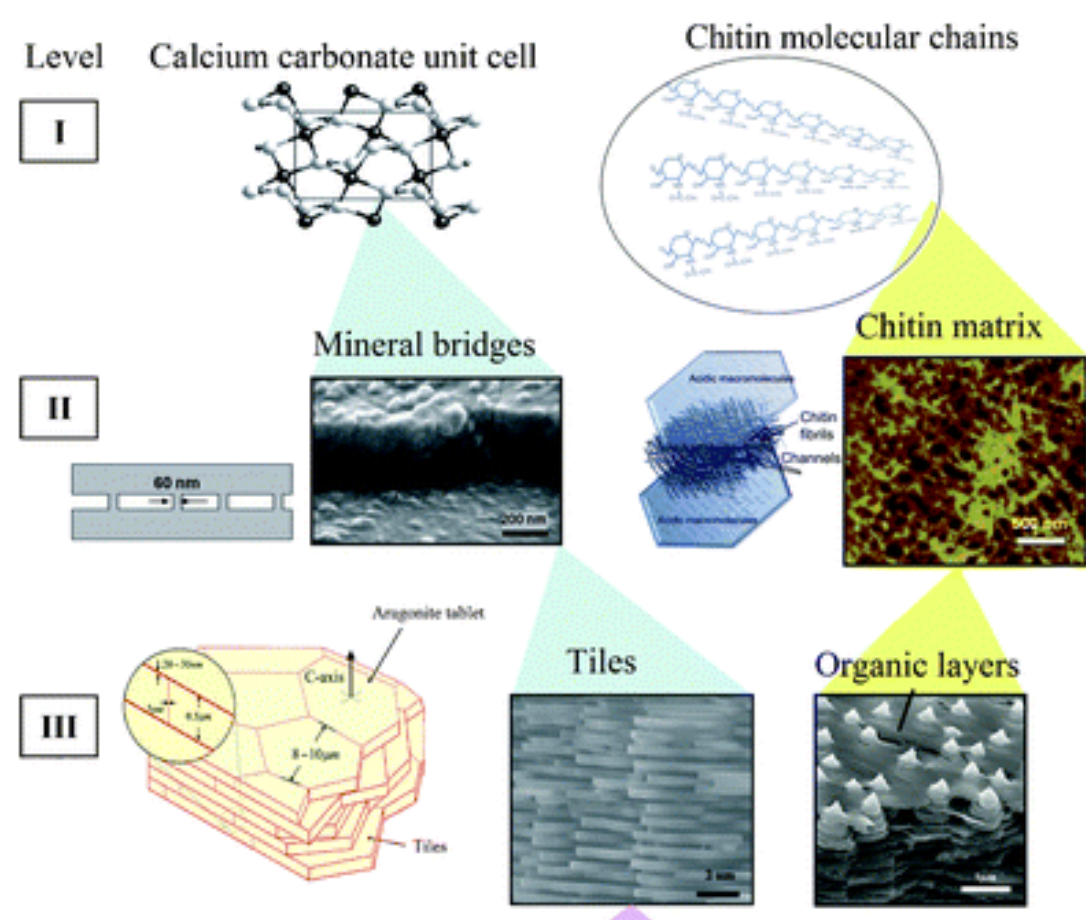

IV Mesolayers
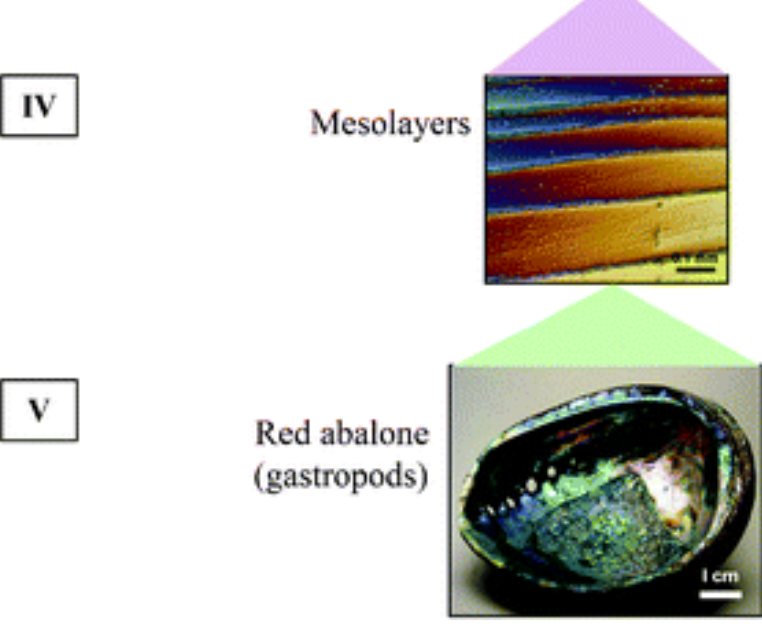

Figure 1.3: Hierarchal organization within the nacreous layer in red abalone (gastropods), reprinted with permission from [41].

At microscale, the brick-mortar structure consists of multiple layers of hard aragonite tiles that are interlaced by thin layers of organic matrix. These tiles are in itself composites that are formed of smaller aragonite platelets glued together by the organic matrix. The brickmortar architecture aids in crack deflection and slip resistance, thus providing high toughness and impact resistance. The mechanical strength of nacre (Young's modulus of about $34 \mathrm{GPa}$ and hardness of $200 \mathrm{~kg} \mathrm{~m}^{-2}$ ) is very high despite the high mineral content and the fact that monolithic aragonite is a very fragile ceramic. It is the close association between the interlacing polymer matrix and the calcium carbonate tablets that results in a strength and toughness 20 - 30 times higher than that of monolithic aragonite [42, 43]. 


\subsection{3b Biomineralization in nacre}

The nanostructure of nacre is considered the simplest of the various forms of calcium carbonate biomineralization found in nature. In nacre, calcium carbonate exists as both calcite and aragonite polymorphs with the latter being dominant in this case. Researchers attempting to replicate this intricate biomineralization under laboratory conditions have narrowed the constituents to the presence of an insoluble nucleating matrix, continuous influx of mineral ions and the interference of negatively charged organic macromolecules (acidic proteins) with the growing crystal nucleus [2].

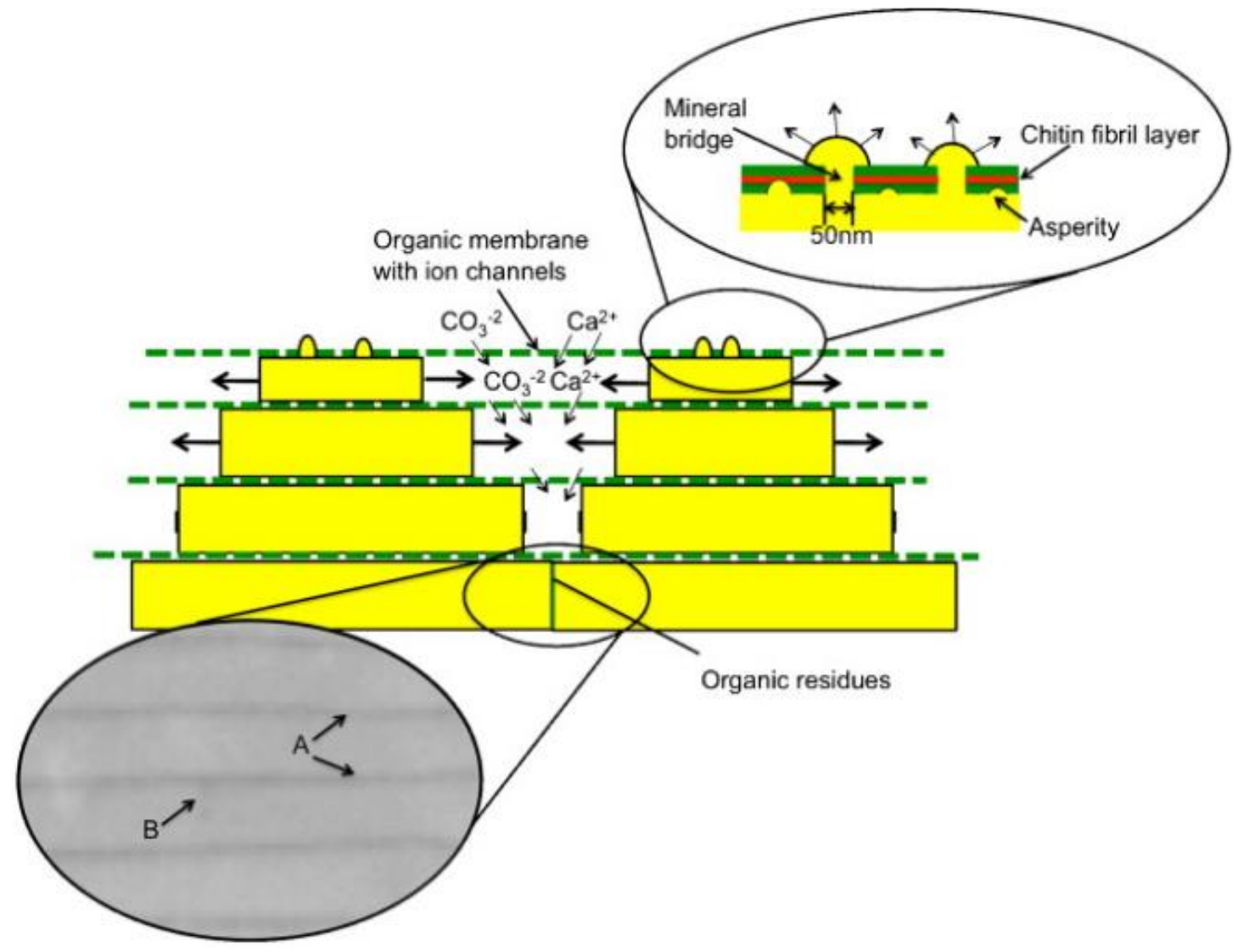

Figure 1.4: A schematic representation of formation of nacreous layer in gastropods in a "Christmas tree" pattern via the formation of mineral bridges. Arrows A and B shows the organic interlayer imaged by SEM and the lateral boundary of tiles. Reprinted with permission from [44].

Literature suggests that mineral molecules initially nucleate in the organic matrix (secreted by the epithelial layers of the mantle) in the form of amorphous calcium carbonate or metastable vaterite and that the nanograins then integrate as the mineralization continues (in association with some chitin fibrils) to form the stable aragonite tiles [45]. The growth 
pattern of the aragonite tablets is described as a "Christmas tree" pattern as shown in Figure 1.4, where the successive layers of tablets are nucleated via mineral bridges even before the underlying layers confluence. The tablets then grow in the horizontal direction until the layer is closed. Figure 1.4 shows a schematic representation of the mineral growth in the nacreous layer of gastropods. The organic layers are permeable to $\mathrm{Ca}^{2+}$ and $\mathrm{CO}_{3}^{2-}$ ions, allowing lateral growth of the tiles until the inter-tile membrane is reached that restricts further growth [44].

It is also suggested that the selection of a thermodynamically less stable calcium carbonate polymorph like aragonite is attributed to the presence of region-specific organic macromolecules. It is suggested that these macromolecules may have structural motifs that match the atomic arrangement of certain planes of the growing crystal nucleus and that their prolonged interactions prevent further nucleation in that direction, hence leading to crystallization and stabilization of a polymorph different from the initial structure $[46,47]$.

Unlike bone, the level of hierarchal organization in nacre is simpler and probably easier to replicate. The majority of the mechanical properties exhibited by nacre are attributed to the brick-mortar motif of the inorganic-organic organization.

The research undertaken for this thesis work is inspired by the biomineralization processes followed in bone and nacre and the porous architecture of trabecular bone in order to develop a synthetic 3D composite structure that uses chitosan and calcium carbonate as the organic and inorganic components respectively. Although calcium phosphates (e.g. hydroxyapatite) are the more immediately obvious choice for development of bone graft materials, in this research, the application of calcium carbonate-based composites with nacre-like morphologies is studied as prospective bone graft materials. This is supported by reports indicating that calcium carbonate is also biocompatible; osteoconductive; has a slower degradation rate than calcium phosphates; and that $\mathrm{CaCO}_{3}$-based composites show slower in vivo biodegradability and higher bone formation after prolonged implantation in rabbit femora in comparison to calcium phosphate-based composites [39].

\subsubsection{Biogenic calcium carbonate}

Calcium carbonate is the most abundant biogenic mineral found in nature. Biological organisms show exquisite control over, among other things, the polymorph selection; localization of crystal nucleation; crystal orientation; crystal size and shape; composition; 
stability; and hierarchical assembly of the various forms of calcium carbonate forming complex structures. In general, it can exist in five different forms, three of which are crystalline, the other two are amorphous. Among the crystalline polymorphs, calcite is the most common followed by aragonite and vaterite, in order of their decreasing thermodynamic stability. In biological systems, calcite and aragonite are commonly found polymorphs however vaterite and amorphous forms are also observed [48].

The calcite structure is described in analogy to the lattice structure of sodium chloride, wherein $\mathrm{Na}^{+}$is replaced by $\mathrm{Ca}^{2+}, \mathrm{Cl}^{-}$is replaced by $\mathrm{CO}_{3}{ }^{2-}$ groups and the unit cell is distorted to form a face-centred rhombohedra. $\mathrm{Ca}^{2+}$ and $\mathrm{CO}_{3}{ }^{2-}$ are in alternate layers perpendicular to the ' $\mathrm{c}$ ' axis. Within a single layer $\mathrm{CO}_{3}{ }^{2-}$ is identically oriented but reverses orientation in adjacent layers (Figure 1.5 (a)). Aragonite on the other hand has an orthorhombic unit cell with the organization of $\mathrm{Ca}^{2+}$ and $\mathrm{CO}_{3}{ }^{2-}$ layers similar to that in calcite, the difference being that a single carbonate layer is split into two layers parallel to the ' $a$ ' axis. This ensures efficient packing of carbonate groups which results in higher density of aragonite compared to thermodynamically stable calcite [2]. Vaterite is the kinetically favoured form of calcium carbonate. The metastable anhydrous calcium carbonate has a hexagonal unit cell.

Morphologically, biogenic calcite exhibits different structures compared to the synthetically prepared perfectly shaped rhombohedra as shown in Figure 1.5 (a). This is due to the continuous interference of the organism involved, which results in modification of the morphologies according to individual biological relevance. Specifically, the factors that influence the crystallization kinetics of $\mathrm{CaCO}_{3}$ resulting in modified morphologies and stabilization of thermodynamically unstable polymorphs in biological systems have narrowed down to be the presence of biopolymers and crystal growth additives (such as amino acids) that may be secreted by the organism. Studies have shown that it is possible to replicate such biological forms of calcium carbonate on a laboratory scale using various additives such as synthetic polymers like polyacrylic acid, polyacrylonitrile, and polystyrene sulfonate; amino acids such as aspartic acid, alanine, lysine and glycine; or in the simplest case addition of magnesium during crystallization of calcium carbonate results in the formation of aragonite spicules rather than rhombohedral calcite [49]. Also, it has been shown that myriads of morphologies of calcium carbonate can be obtained by varying the crystallization reaction conditions such as $\mathrm{pH}$, reactant concentration, time, and temperature $[46,47,50]$. 
In the research presented here the aim is to replicate nacre's brick-mortar architecture in laboratory conditions using 3D chitosan hydrogel-based scaffolds in conjunction with polyacrylic acid as the crystal growth modifier and a mineralization technique known as the McGrath method. Previously, this technique has been effectively used to replicate the structure of nacre using a 2D chitosan hydrogel-based film (explained further in Section 1.2.6).
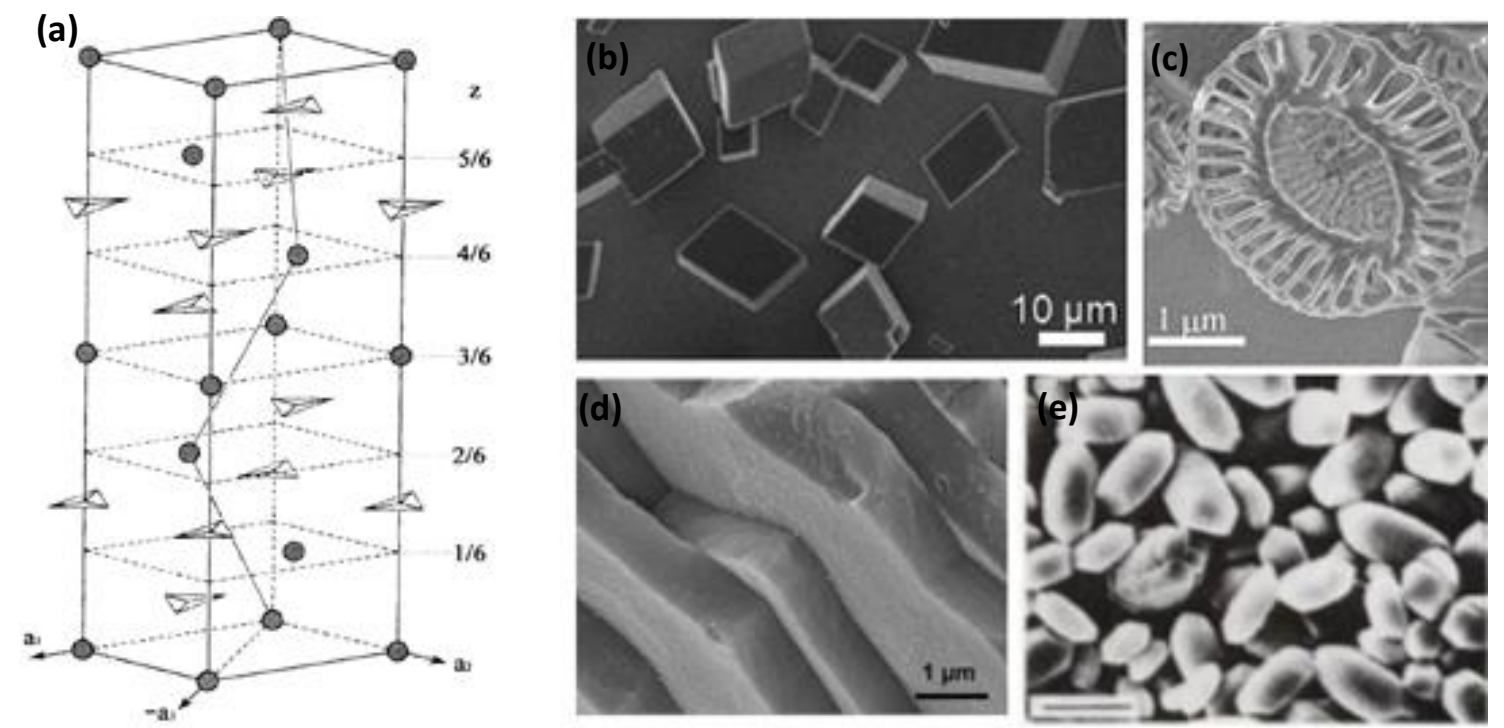

Figure 1.5: (a) The crystallographic unit cell of calcite [2]; SEM images of biogenic calcium carbonate of different morphologies as in (b) synthetic rhombohedral calcium carbonate [51]; (c) calcite in coccolithophorids [36]; (d) aragonite tablets in molluscs [52]; and (e) calcite crystals from the human inner ear (scale bar is $8 \mu \mathrm{m}$ ) [37]. Reprinted with permission.

\subsubsection{Chitosan hydrogels for orthopaedic applications}

Over the years a large number of synthetic polymers (such as poly(propylene fumarate), poly(ethylene oxide)-polybutylene terephthalate, polycaprolactone, polylactidepolyglycolide [53-56]); and natural composites of collagen, tricalcium phosphates, nano hydroxyapatite, gelatin [57-59] have been studied as biocompatible materials to fabricate bio-mimics of bone. In this project chitosan hydrogel-based scaffolds are studied as a prospective material to fabricate $3 \mathrm{D}$ porous composites towards future bone tissue engineering applications.

Chitosan is an amino-polysaccharide which can be chemically modified to generate novel properties that are applicable in biomedical research. It is synthetically derived from chitin 
(often extracted from prawn shells) via partial deacetylation in the presence of an alkali, resulting in a polysaccharide which is soluble only under acidic conditions. In acidic solution, chitosan forms a hydrogel structure via physical interaction (hydrogen bonding) between the hydrophilic groups $\left(-\mathrm{OH},-\mathrm{NH}_{2}\right)$ on the polymer chain and water molecules. Hydrogels are an important category of chitosan-based materials useful for application in bone tissue engineering applications due to their structural similarity to extra cellular matrix (ECM). These chitosan hydrogels also exhibit the tendency to swell with high water absorption properties similar to biological tissues. The swelling tendency can however vary with the degree of deacetylation of the chitosan polymer chains [60]. Unlike the rigid crystallinity of chitosan in the solid state due to the regularly arranged hydroxyl and amino groups in the $\beta(1,4)$-linked D-glucosamine repeating units, in solution, hydrogen bonding leads to the formation of microfibrils, which depending on the chitosan concentration yields a state that is similar to the protein structures in ECM [61]. This, along with a high surfaceto-volume ratio, which promotes diffusion owing to its extensive pore interconnectivity, makes chitosan hydrogels good candidates in developing biocompatible implants.

Studies have also shown that chitosan hydrogel-based scaffolds evoke minimal foreignbody response and fibrous encapsulation [61]. It also shows excellent biodegradation, cytocompatibility, blood compatibility, and antimicrobial activity without any abnormal inflammatory reactions. The cationic nature of chitosan at acidic $\mathrm{pH}$ is especially advantageous in forming polyelectrolyte complexes with anionic biological macromolecules, thus presenting an opportunity for improved cell adhesion and bone tissue formation in vivo [62]. All of these properties suggest that chitosan hydrogels show good prospects for in vivo applications. However, for orthopaedic applications, it is very important that structures based on chitosan hydrogels exhibit mechanical strength compatibility to the implant site. Considerable research is underway in this area in terms of improving the mechanical properties of chitosan hydrogel-based structures and some studies have shown that this can be done via physical and chemical crosslinking [62-65]. Crosslinking can be in the form of covalent crosslinking such as when using glutaraldehyde or physical crosslinking via non-covalent interactions such as physical entanglements or ionic bonding (such as deprotonation of the chitosan chain in the presence of sodium hydroxide which prevents dissolution of the hydrogels).

The mechanical strength of chitosan hydrogels can also be improved by combining these with other scaffold materials to form composites. Studies have shown that composites of 
chitosan and synthetic, natural polymers (gelatin, chondritin sulfate) or inorganic materials such as calcium phosphates or HAP composites depict improved structural stability and mechanical properties in comparison to pure chitosan hydrogel-based structures [66]. In this research, introduction of calcium carbonate crystallites of a desired morphology inspired by the aragonite tablets in nacre is studied as an effective technique to augment the mechanical strength of physically crosslinked chitosan hydrogel-based scaffolds. The McGrath mineralization method, employed for this purpose, is a matrix-induced mineralization technique which facilitates mineral growth in association with the hydrogel matrix. This method is inspired by the mineralization that occurs in bone and nacre and the resulting enhanced interaction between the matrix and the minerals is envisaged to improve the mechanical resistance to stress of the resultant composites.

\subsubsection{Matrix-induced mineral growth: A strategy to improve mechanical properties}

Mechanical properties of pure chitosan hydrogel-based scaffolds are poor. At the highest chitosan concentration (12 wt\%), pure chitosan scaffolds have an average compressive modulus of $17.99 \pm 0.11 \mathrm{MPa}$, which is significantly less than that of human cancellous bone (100-500 MPa) [67]. In order to improve these properties, minerals can be introduced as filler particles such that the stress distribution is modified. Minerals can either be dispersed in the organic component and then cast into a mould to form a 3D structure or can be grown within the organic matrix of a 3D structure via induced mineral growth by controlled infusion and reaction of moderately concentrated ionic precursors. Chapter 3 and 4 of this thesis gives an effective comparison between chitosan-calcium carbonate composites developed using different matrix-induced mineralization techniques (biomimetic mineralization techniques) and mechanical blending of individual components.

This research primarily focusses on the applicability of a mineralization process, the McGrath mineralization method, previously developed by the McGrath research group, in an effort to replicate the elements of the nanostructure of nacre in laboratory conditions [7]. This method is a combination of two well-established mineralization techniques: the alternate soaking method [35] and the one developed by Kitano et al [68]. The alternate soaking method involving repeated soaking of an organic scaffold in precursor mineral solutions, facilitating transport of ions within the matrix, was also successfully devised to introduce mineral growth within an organic matrix [35]. In the latter process, an organic template is soaked in supersaturated solution of calcium carbonate for prolonged periods of 
time, it relies on $\mathrm{pH}$ disruptions to initiate nucleation on and within an organic matrix or solution [68].

In order to mineralize 2D chitosan hydrogel-based films via the McGrath method, the crosslinked film (physical crosslinking via $\mathrm{NH}_{3}{ }^{+}$groups present along the chitosan chains), is first alternately soaked (e.g. for $1 \mathrm{~h}$ each) in solutions of each of the precursor ions, inducing heterogenous nucleation of calcium carbonate particles (nano to micron-sized). This film is then soaked for a prolonged period of time (e.g. for one week) in a saturated solution of calcium carbonate, herein called the Kitano solution, allowing further growth of the crystallites [7]. The morphology of the calcium carbonate crystallites can be modified to resemble the circular pancake-like structure, observed at the early stages of nacre biomineralization, by introducing polyacrylic acid to the mineralization media as the crystal growth modifier (refer to Chapter 3, Figure 3.1) [69]. The effect of various crystal growth modifiers such as polyacrylic acid, poly L-glutamic acid, poly L-aspartic acid and their corresponding monomers on the morphology, polymorph selection and composition of the resultant inorganic-organic composite of chitosan and $\mathrm{CaCO}_{3}$ was also studied in detail [11]. The results suggested that the McGrath method is a versatile mineralization method that can be used to achieve calcium carbonate or calcium phosphate growth within 2D carbohydrate (chitosan) or protein-based (gelatin) films [11]. Composites of $\mathrm{CaCO}_{3}$, magnesium enriched calcium carbonate and calcium phosphate were fabricated using this method [8]. Mineralized 2D chitosan hydrogel-based films showed an effective tensile strength of the same order of magnitude as that of nacre [7].

This research investigates effectively translating the synthetic biomineralization achieved in 2D chitosan-based films to 3D porous chitosan-based scaffolds using the McGrath method with polyacrylic acid as the crystal growth modifier.

\subsubsection{Porosity and its implications on mechanical strength of engineered scaffolds}

Control over the porosity and pore interconnectivity is essential in imparting different properties in an engineered scaffold. Literature suggests that an optimum pore size of $5 \mu \mathrm{m}$ is preferable for neovascularization, $5-15 \mu \mathrm{m}$ for fibroblast ingrowth, $20-125 \mu \mathrm{m}$ for regeneration of adult mammalian skin, 100 - $350 \mu \mathrm{m}$ can initiate bone regeneration, 40 $100 \mu \mathrm{m}$ for osteoid ingrowth, and $20 \mu \mathrm{m}$ for ingrowth of hepatocytes [27, 70]. Extensive studies have been performed on a wide range of scaffold materials for bone as well as cartilage regeneration. These reveal that the mechanical properties of scaffolds is also 
influenced by the variation of architectural parameters, such as degree of porosity [71], pore size [72], pore shape [73], fibre diameter and fibre spacing [74, 75]. For example, polyesterbased sponge scaffolds of poly-L-lactide acid (PLLA) (300 $\mu$ m pores and 90 - 95\% porosity) and fibrous scaffolds ( $1 \mathrm{~mm}$ pores and $85 \%$ porosity) exhibit mechanical properties (compression Young's modulus) similar to that of natural cartilage which is about 0.84 - 3 $\mathrm{MPa}$ for a $70 \mathrm{~kg}$ person [71]. Another study indicated that the mechanical properties of blended polycaprolactone and poly(lactic-co-glycolic acid)-based scaffolds varied with the pore structure and layer stacking direction [76]. It was found that the compressive strengths of the lattice, stagger, and triangle-type unit cell-based scaffolds were 6.05, 7.43, and 9.81 $\mathrm{MPa}$, respectively with the compressive strength and modulus of the triangle-type scaffold (178 MPa) being the highest and within the same order of magnitude as that of human tubercular bone. Another study on the effect of variation of pore size on the mechanical properties of woven chitosan-hydroxyapatite copolymeric fibrous scaffolds showed that chondrocyte-seeded scaffolds with small pores $(100 \mu \mathrm{m}$, lower porosity) had a higher compression modulus than cell seeded scaffolds with 200 or $400 \mu \mathrm{m}$ pores. However, after 28 days of incubation, the scaffolds with the smaller pore sizes had reduced compression modulus [77]. Pore interconnectivity, while not necessarily impacting mechanical strength, is important in ensuring cellular mobility/migration, nutrient inflow and exchange when implanted in vivo. Literature also suggests that scaffolds designed with a combination of macro and micropores can facilitate both improved bone formation and vascularization in vivo as a varied range of pore sizes enable different functionalities as suggested earlier [78].

Although chitosan hydrogel-based scaffolds have poor mechanical properties, the combination of different pore sizes and the introduced mineral component (calcium carbonate) is expected to increase its strength to an order comparable to natural bone. Also, its long-range porosity, varying from macro (introduced via 3D printing) to nanoscale (intrinsic pores in chitosan hydrogel), is expected to promote cell growth, proliferation and vasculature in vivo.

\subsubsection{Fabrication of 3D porous chitosan hydrogel-based scaffolds}

Studies suggest that a 3D microenvironment, similar to the natural state, is better suited for enhanced cell interactions, adhesion and growth compared to 2D culture systems for in vivo applications [79-82]. This is due to the fact that in in vivo conditions cells are immersed in a 3D network of a complex extracellular environment which is highly porous and has a 
topography engineered to the nanoscale (which is responsible for inducing the cells to differentiate in response to specific physical and chemical signals). A 2D culture system is too simple to mimic this complex native micro-environment and thus, 3D systems are being researched more for in vivo biomedical applications. It is also important that this $3 \mathrm{D}$ structure is a well-connected system internally (interconnected porous network) that facilitates cell mobility, vascularisation, flow of nutrient and ion exchange.

Several methods are being developed to fabricate 3D porous scaffolds using hydrogels ranging from chemical/gas foaming, solvent casting, particle/salt leaching, freeze-drying, thermally-induced phase separation to foam-gel methods [62]. Chitosan scaffolds have been reported to be synthesised via all of the above techniques but have encountered certain problems as discussed below.

- Phase separation and lyophilisation is the most extensively used technique. Here chitosan, dissolved in acetic acid, solution is poured into a mould and subjected to controlled freezing. Upon freezing, ice crystals phase-separate from the acetate salt. These ice crystals further sublimate in a freeze-drying step, resulting in a porous structure. The final porous scaffold is neutralised using sodium hydroxide and hydrated in ethanol. In this process, there is limited control over the pore dimension, distribution and interconnectivity as it is affected by parameters such as the freezing temperature, thermal gradients, and the polymer concentration [83]. A disadvantage of this technique is that freeze-drying causes the formation of a surface skin at the scaffold-air interface due to an interfacial tension effect during solvent evaporation which collapses the pores on the surface and hence hinders diffusibility of the scaffold.

- In order to eliminate the side effects of freeze-drying, the freeze-gelation technique was introduced, where the chitosan scaffold undergoes gelation in an ethanolic sodium hydroxide solution (deprotonation of chitosan polymer chain occurs) at $-20{ }^{\circ} \mathrm{C}$. The pores filled with ethanol can be air-dried hence avoiding the freeze-drying step [84].

- Particulate leaching is yet another method in which a sacrificial porogen is mixed with the chitosan solution, which is then leached out using a suitable solvent following the lyophilisation step [85]. This results in two levels of porosity one generated via the sublimation of ice crystals during lyophilisation and the other from leaching of the porogens. Commonly used porogens are inexpensive salts and sugars, but the process is labour extensive. Although the pore size and extent of porosity can be controlled by the 
type, size and concentration of the porogens used, the pore distribution within the 3D structure thus generated cannot be controlled.

- Gas foaming is a technique wherein porous networks result from the nucleation and growth of gas bubbles within a polymeric system. Gas bubbles can be nucleated and coalesced within a polymer solution by (1) addition of an effervescent mixture of salts in the polymer solution and later reacting them in a suitable solution or (2) by foaming the polymer solution at high pressure $[86,87]$. The advantage of this technique is that it does not involve the use of any organic solvents, thus reducing the chances of in vivo toxicity. The disadvantage being that the pore formation and distribution depend on the rate of nucleation and gas diffusion, which can be hard to control.

Although most of the above procedures offer high degrees of porosity, the architecture of the scaffolds thus generated are not reproducible to precise designs for in vivo applications such as bone tissue engineering. Furthermore, the complex hierarchal organisation found in bone cannot be replicated using the methods mentioned above. New techniques collectively called solid free-form fabrication, such as 3D printing using nozzle extrusion-based systems, offer specific control over the micro and macro architecture of the scaffold via a computer controlled $\mathrm{x}, \mathrm{y}, \mathrm{z}$ table, robotic dispensing technology. The printer input files can be designed with specific structural features using CAD tools, hence offering reproducibility [88]. This additive manufacturing technology allows layer-by-layer development of complex structures with high degree of precision, similar to the natural systems. The precision and specificity in architectural features that can be achieved through CAD-based 3D printing offer control over balancing the load bearing capacity and tissue regeneration requirements of in vivo bone graft. These designs can be tailored specifically to achieve a particular topography, which is advantageous as research suggests that a scaffold's topography/architecture influences cell differentiation and hence regeneration at the implant site [89]. Current technology also allows micro-computed tomography (micro-CT) or magnetic resonance imaging (MRI) images of natural bone to be converted to printer compatible formats (such as .STL), thus facilitating fabrication of exact replicas of the natural structures. This will ensure perfect anatomic compatibility between the implant and the native bone $[30,90]$. In Chapter 2, the application of this technique in this research work is explained in detail.

In summary, this work investigates the possibility of fabricating 3D porous structures using chitosan hydrogels as the printing ink in a custom-designed nozzle extrusion-based 3D 
printer. The applicability of the McGrath method is then investigated as a matrix-induced mineralization technique for developing composites of chitosan and calcium carbonate. The biological relevance and mechanical properties of the thus fabricated composite is then studied to identify the prospect of this material as a future bone graft. 


\subsection{Thesis structure}

In Chapter 2, the technique employed to fabricate 3D chitosan hydrogel-based scaffolds using nozzle extrusion-based 3D printing technology employing chitosan hydrogel as the printing ink, is described. The properties of our custom-designed 3D printer, rheology of the hydrogel, the chosen printing parameters and the protocol developed to fabricate a stable 3D porous chitosan hydrogel-based scaffold is discussed. Further, various techniques used to dry the as-fabricated scaffolds and their effects on the scaffold structure, porosity and swelling behaviour in a physiologically relevant solvent are explained. SEM, Cryo-SEM, rheometery and micro-CT were employed for these analyses.

In Chapter 3, the strategies adopted to successfully translate the nacre-like mineralization previously achieved by the McGrath group on 2D chitosan hydrogel-based films, to the 3D printed chitosan hydrogel-based scaffolds in order to develop 3D chitosan-calcium carbonate composites are described. The modifications incorporated in the McGrath mineralization method for the above and for optimising the morphology and distribution of calcium carbonate crystallites in the form of the desired pancake-like structure throughout the 3D scaffold is described. SEM, XRD, EDS, TGA and micro-CT analysis were employed for these analyses.

In Chapter 4, the advantages and disadvantages of an alternative method for fabrication of 3D chitosan-calcium carbonate composites via 3D printing using chitosan-calcium carbonate blended hydrogels as the printing ink is described. The tunability of the properties of the resultant composites such as the morphology of the calcium carbonate crystallites and the strength of the interaction between the organic matrix and the crystallites is analysed using SEM, XRD and TGA analyses.

The mechanical properties of chitosan-calcium carbonate composites fabricated by mineralization of chitosan hydrogel-based scaffolds via the McGrath are discussed in Chapter 5. The behaviour of the composites under compressive stress is studied via uniaxial compression testing, dynamic mechanical analysis and nanoindentation testing. The effect of porosity, total mineral content, and the morphology and size of the crystallites on the mechanical properties of the composites are explained.

In Chapter 6 the in vitro biological performance of chitosan hydrogel-based scaffolds and composites is discussed. Specifically, the osteoblast cell viability, proliferation and 
adhesion in the presence of the 3D printed chitosan hydrogel-based scaffolds and the composites are explained.

Chapter 7 concludes this thesis highlighting the main outcomes and future of this research. 


\subsection{Bibliography}

1. Wegst, U.G., H. Bai, E. Saiz, A.P. Tomsia, and R.O. Ritchie, Bioinspired structural materials. Nature Materials, 2015. 14(1): p. 23-36.

2. Meldrum, F., Calcium carbonate in biomineralization and biomimetic chemistry. International Materials Reviews, 2003. 48(3): p. 187-224.

3. Podsiadlo, P., S. Paternel, J.-M. Rouillard, Z. Zhang, J. Lee, J.-W. Lee, E. Gulari, and N.A. Kotov, Layer-by-layer assembly of nacre-like nanostructured composites with antimicrobial properties. Langmuir, 2005. 21(25): p. 11915-11921.

4. Oaki, Y., A. Kotachi, T. Miura, and H. Imai, Bridged nanocrystals in biominerals and their biomimetics: classical yet modern crystal growth on the nanoscale. Advanced Functional Materials, 2006. 16(12): p. 1633-1639.

5. Verma, D., K.S. Katti, D.R. Katti, and B. Mohanty, Mechanical response and multilevel structure of biomimetic hydroxyapatite/polygalacturonic/chitosan nanocomposites. Materials Science and Engineering: C, 2008. 28(3): p. 399-405.

6. Sellinger, A., P.M. Weiss, A. Nguyen, Y. Lu, R.A. Assink, W. Gong, and C.J. Brinker, Continuous self-assembly of organic-inorganic nanocomposite coatings that mimic nacre. Nature, 1998. 394(6690): p. 256-260.

7. Munro, N.H., D.W. Green, A. Dangerfield, and K.M. McGrath, Biomimetic mineralization of polymeric scaffolds using a combined soaking and Kitano approach. Dalton Transactions, 2011. 40(36): p. 9259-9268.

8. Munro, N.H. and K.M. McGrath, Biomimetic mineralization of polymeric scaffolds using a combined soaking approach: adaptation with various mineral salts. Dalton Transactions, 2011. 40(36): p. 9269-9275.

9. Munro, N.H., D.W. Green, and K.M. McGrath, In situ continuous growth formation of synthetic biominerals. Chemical Communications, 2013. 49(33): p. 3407-3409.

10. Mucadam, R.M.-M., Architecture of a nanocrystalline biomineralised shell : a thesis submitted to the Victoria University of Wellington 2014.

11. Afsar, S., Carbohydrate/protein hydrogels as responsive scaffolds in controlling inorganic crystallization : a thesis submitted to the Victoria University of Wellington 2016.

12. Janicki, P. and G. Schmidmaier, What should be the characteristics of the ideal bone graft substitute? Combining scaffolds with growth factors and/or stem cells. Injury, 2011. 42: p. S77-S81. 
13. Campana, V., G. Milano, E. Pagano, M. Barba, C. Cicione, G. Salonna, W. Lattanzi, and G. Logroscino, Bone substitutes in orthopaedic surgery: from basic science to clinical practice. Journal of Materials Science: Materials in Medicine, 2014. 25(10): p. 2445-2461.

14. Werier, J., Focus On Bone graft substitutes in oncology, paediatrics, and hip arthroplasty. Br. Editor. Soc. Bone Jt. Surg, 2012: p. 34-35.

15. Schlickewei, W. and C. Schlickewei. The use of bone substitutes in the treatment of bone defects-the clinical view and history. in Macromolecular Symposia. 2007. Wiley Online Library.

16. Hung, N.N., Basic Knowledge of Bone Grafting, in Bone Grafting. 2012, InTech.

17. Amini, A.R., C.T. Laurencin, and S.P. Nukavarapu, Bone tissue engineering: recent advances and challenges. Critical Reviews ${ }^{\mathrm{TM}}$ in Biomedical Engineering, 2012. 40(5).

18. Costa-Pinto, A.R., R.L. Reis, and N.M. Neves, Scaffolds based bone tissue engineering: the role of chitosan. Tissue Engineering Part B: Reviews, 2011. 17(5): p. 331-347.

19. Laurencin, C., Y. Khan, and S.F. El-Amin, Bone graft substitutes. 2006.

20. Friedman, C.D., P.D. Costantino, S. Takagi, and L.C. Chow, BoneSource ${ }^{\mathrm{TM}}$ hydroxyapatite cement: a novel biomaterial for craniofacial skeletal tissue engineering and reconstruction. Journal of Biomedical Materials Research Part A, 1998. 43(4): p. 428-432.

21. Peltier, L.F. and R. Jones, Treatment of unicameral bone cysts by curettage and packing with plaster-of-Paris pellets. Journal of Bone and Joint Surgery, 1978. 60(6): p. 820822.

22. Nandi, S., S. Roy, P. Mukherjee, B. Kundu, D. De, and D. Basu, Orthopaedic applications of bone graft \& graft substitutes: a review. 2010.

23. Scabbia, A. and L. Trombelli, A comparative study on the use of a $H A /$ collagen/chondroitin sulphate biomaterial (Biostite $\left.{ }^{\circledR}\right)$ and a bovine-derived HA xenograft (Bio-Oss $\left.{ }^{\circledR}\right)$ in the treatment of deep intra-osseous defects. Journal of Clinical Periodontology, 2004. 31(5): p. 348-355.

24. Serre, C., M. Papillard, P. Chavassieux, and G. Boivin, In vitro induction of a calcifying matrix by biomaterials constituted of collagen and/or hydroxyapatite: an ultrastructural comparison of three types of biomaterials. Biomaterials, 1993. 14(2): p. 97-106. 
25. Yamauchi, K., T. Goda, N. Takeuchi, H. Einaga, and T. Tanabe, Preparation of collagen/calcium phosphate multilayer sheet using enzymatic mineralization. Biomaterials, 2004. 25(24): p. 5481-5489.

26. Li, B., Q. Hu, X. Qian, Z. Fang, and J. Shen, Bioabsorbable chitosan/hydroxyapatite composite rod prepared by in situ precipitation for internal fixation of bone fracture. Acta Polymerica Sinica, 2002(6): p. 828-833.

27. Venkatesan, J. and S.-K. Kim, Chitosan composites for bone tissue engineering - an overview. Marine Drugs, 2010. 8(8): p. 2252-2266.

28. Athanasiou, K., C.-F. Zhu, D. Lanctot, C. Agrawal, and X. Wang, Fundamentals of biomechanics in tissue engineering of bone. Tissue Engineering, 2000. 6(4): p. 361381.

29. Ajayan, P.M., L.S. Schadler, and P.V. Braun, Nanocomposite science and technology. 2006: John Wiley \& Sons.

30. Hollinger, J.O., T.A. Einhorn, B. Doll, and C. Sfeir, Bone tissue engineering. 2004: CRC Press.

31. Rho, J.-Y., L. Kuhn-Spearing, and P. Zioupos, Mechanical properties and the hierarchical structure of bone. Medical Engineering \& Physics, 1998. 20(2): p. 92-102.

32. Henkel, J., M.A. Woodruff, D.R. Epari, R. Steck, V. Glatt, I.C. Dickinson, P.F. Choong, M.A. Schuetz, and D.W. Hutmacher, Bone regeneration based on tissue engineering conceptions - a 21st century perspective. Bone Research, 2013. 1: p. 216-248.

33. Lee, S., M. Porter, S. Wasko, G. Lau, P.-Y. Chen, E.E. Novitskaya, A.P. Tomsia, A. Almutairi, M.A. Meyers, and J. McKittrick, Potential bone replacement materials prepared by two methods. MRS Online Proceedings Library Archive, 2012. 1418.

34. Weiner, S. and H.D. Wagner, The material bone: structure-mechanical function relations. Annual Review of Materials Science, 1998. 28(1): p. 271-298.

35. Ma, P.X. and J.-W. Choi, Biodegradable polymer scaffolds with well-defined interconnected spherical pore network. Tissue Engineering, 2001. 7(1): p. 23-33.

36. Kayano, K., K. Saruwatari, T. Kogure, and Y. Shiraiwa, Effect of coccolith polysaccharides isolated from the coccolithophorid, Emiliania huxleyi, on calcite crystal formation in in vitro $\mathrm{CaCO}_{3}$ crystallization. Marine Biotechnology, 2011. 13(1): p. 83-92.

37. Mann, S., Biomineralization: principles and concepts in bioinorganic materials chemistry. Vol. 5. 2001: Oxford University Press on Demand. 
38. Sun, J. and B. Bhushan, Hierarchical structure and mechanical properties of nacre: a review. RSC Advances, 2012. 2(20): p. 7617-7632.

39. He, F., W. Ren, X. Tian, W. Liu, S. Wu, and X. Chen, Comparative study on in vivo response of porous calcium carbonate composite ceramic and biphasic calcium phosphate ceramic. Materials Science and Engineering: C, 2016. 64: p. 117-123.

40. Sadeghi, H., D.M. Espino, and D.E. Shepherd, Variation in viscoelastic properties of bovine articular cartilage below, up to and above healthy gait-relevant loading frequencies. Proceedings of the Institution of Mechanical Engineers, Part H: Journal of Engineering in Medicine, 2015. 229(2): p. 115-123.

41. Meyers, M.A., P.-Y. Chen, M.I. Lopez, Y. Seki, and A.Y. Lin, Biological materials: A materials science approach. Journal of the Mechanical Behavior of Biomedical Materials, 2011. 4(5): p. 626-657.

42. Barthelat, F., C.-M. Li, C. Comi, and H.D. Espinosa, Mechanical properties of nacre constituents and their impact on mechanical performance. Journal of Materials Research, 2006. 21(8): p. 1977-1986.

43. Currey, J.D., P. Zioupos, D. Peter, and A. Casinos, Mechanical properties of nacre and highly mineralized bone. Proceedings of the Royal Society of London B: Biological Sciences, 2001. 268(1462): p. 107-111.

44. Meyers, M., C. Lim, A. Li, B.H. Nizam, E. Tan, Y. Seki, and J. McKittrick, The role of organic intertile layer in abalone nacre. Materials Science and Engineering: C, 2009. 29(8): p. 2398-2410.

45. Qiao, L., Q. Feng, and S. Lu, In vitro growth of nacre-like tablet forming: from amorphous calcium carbonate, nanostacks to hexagonal tablets. Crystal Growth and Design, 2008. 8(5): p. 1509-1514.

46. Altay, E., T. Shahwan, and M. Tanoğlu, Morphosynthesis of $\mathrm{CaCO}_{3}$ at different reaction temperatures and the effects of PDDA, CTAB, and EDTA on the particle morphology and polymorph stability. Powder Technology, 2007. 178(3): p. 194-202.

47. Declet, A., E. Reyes, and O. Suárez, Calcium Carbonate Precipitation: A Review Of The Carbonate Crystallization Process And Applications In Bioinspired Composites. Reviews on Advanced Materials Science, 2016. 44(1).

48. Addadi, L., S. Raz, and S. Weiner, Taking advantage of disorder: amorphous calcium carbonate and its roles in biomineralization. Advanced Materials, 2003. 15(12): p. 959970. 
49. Butler, M.F., N. Glaser, A.C. Weaver, M. Kirkland, and M. Heppenstall-Butler, Calcium carbonate crystallization in the presence of biopolymers. Crystal Growth \& Design, 2006. 6(3): p. 781-794.

50. Tai, C.Y. and F.B. Chen, Polymorphism of $\mathrm{CaCO}$, precipitated in a constantcomposition environment. AIChE Journal, 1998. 44(8): p. 1790-1798.

51. Shi, S., L. Cai, X. Liu, H. Li, Y. Gao, Q. Wu, J. Yi, X. Song, and G. Zhang, Bioinspired fabrication of macroporous calcium carbonate crystals mediated by thermoresponsive copolymers. RSC Advances, 2017. 7(70): p. 44505-44513.

52. Ma, Y., S. Berland, J.-P. Andrieu, Q. Feng, and L. Bédouet, What is the difference in organic matrix of aragonite vs. vaterite polymorph in natural shell and pearl? Study of the pearl-forming freshwater bivalve mollusc Hyriopsis cumingii. Materials Science and Engineering: C, 2013. 33(3): p. 1521-1529.

53. Peter, S., M. Miller, A. Yasko, M. Yaszemski, and A. Mikos, Polymer concepts in tissue engineering. Journal of Biomedical Materials Research, 1998. 43(4): p. 422-427.

54. Sakkers, R., R. Dalmeyer, J. de Wijn, and C. Van Blitterswijk, Use of bone-bonding hydrogel copolymers in bone: An in vitro and in vivo study of expanding PEO-PBT copolymers in goat femora. Journal of Biomedical Materials Research, 2000. 49(3): p. 312-318.

55. Hutmacher, D.W., T. Schantz, I. Zein, K.W. Ng, S.H. Teoh, and K.C. Tan, Mechanical properties and cell cultural response of polycaprolactone scaffolds designed and fabricated via fused deposition modeling. Journal of Biomedical Materials Research, 2001. 55(2): p. 203-216.

56. Middleton, J.C. and A.J. Tipton, Synthetic biodegradable polymers as orthopedic devices. Biomaterials, 2000. 21(23): p. 2335-2346.

57. Tarafder, S., V.K. Balla, N.M. Davies, A. Bandyopadhyay, and S. Bose, Microwavesintered 3D printed tricalcium phosphate scaffolds for bone tissue engineering. Journal of Tissue Engineering and Regenerative Medicine, 2013. 7(8): p. 631-641.

58. Liu, C., Z. Xia, Z. Han, P. Hulley, J. Triffitt, and J. Czernuszka, Novel 3D collagen scaffolds fabricated by indirect printing technique for tissue engineering. Journal of Biomedical Materials Research Part B: Applied Biomaterials, 2008. 85(2): p. 519-528.

59. Leukers, B., H. Gülkan, S.H. Irsen, S. Milz, C. Tille, M. Schieker, and H. Seitz, Hydroxyapatite scaffolds for bone tissue engineering made by $3 D$ printing. Journal of Materials Science: Materials in Medicine, 2005. 16(12): p. 1121-1124. 
60. Ahmadi, F., Z. Oveisi, S.M. Samani, and Z. Amoozgar, Chitosan based hydrogels: characteristics and pharmaceutical applications. Research in Pharmaceutical Sciences, 2015. 10(1): p. 1.

61. Wang, C., J. Li, and F. Yao, Application of Chitosan-Based Biomaterials in Tissue Engineering. Chitosan-Based Hydrogels: Functions and Applications, 2011: p. 407.

62. Levengood, S.K.L. and M. Zhang, Chitosan-based scaffolds for bone tissue engineering. Journal of Materials Chemistry B, 2014. 2(21): p. 3161-3184.

63. Cho, B.C., J.W. Park, B.S. Baik, and I.C. Kwon, The role of hyaluronic acid, chitosan, and calcium sulfate and their combined effect on early bony consolidation in distraction osteogenesis of a canine model. Journal of Craniofacial Surgery, 2002. 13(6): p. 783793.

64. Pillai, C., W. Paul, and C.P. Sharma, Chitin and chitosan polymers: Chemistry, solubility and fiber formation. Progress in Polymer Science, 2009. 34(7): p. 641-678.

65. Kean, T. and M. Thanou, Biodegradation, biodistribution and toxicity of chitosan. Advanced Drug Delivery Reviews, 2010. 62(1): p. 3-11.

66. Chesnutt, B.M., A.M. Viano, Y. Yuan, Y. Yang, T. Guda, M.R. Appleford, J.L. Ong, W.O. Haggard, and J.D. Bumgardner, Design and characterization of a novel chitosan/nanocrystalline calcium phosphate composite scaffold for bone regeneration. Journal of Biomedical Materials Research Part A, 2009. 88(2): p. 491-502.

67. Jana, S., S.J. Florczyk, M. Leung, and M. Zhang, High-strength pristine porous chitosan scaffolds for tissue engineering. Journal of Materials Chemistry, 2012. 22(13): p. 6291-6299.

68. Kuttappan, S., D. Mathew, and M.B. Nair, Biomimetic composite scaffolds containing bioceramics and collagen/gelatin for bone tissue engineering-A mini review. International Journal of Biological Macromolecules, 2016. 93: p. 1390-1401.

69. Williams, J.M., A. Adewunmi, R.M. Schek, C.L. Flanagan, P.H. Krebsbach, S.E. Feinberg, S.J. Hollister, and S. Das, Bone tissue engineering using polycaprolactone scaffolds fabricated via selective laser sintering. Biomaterials, 2005. 26(23): p. 48174827.

70. Whang, K., K. Healy, D. Elenz, E. Nam, D. Tsai, C. Thomas, G. Nuber, F. Glorieux, R. Travers, and S. Sprague, Engineering bone regeneration with bioabsorbable scaffolds with novel microarchitecture. Tissue Engineering, 1999. 5(1): p. 35-51. 
71. Izadifar, Z., X. Chen, and W. Kulyk, Strategic design and fabrication of engineered scaffolds for articular cartilage repair. Journal of Functional Biomaterials, 2012. 3(4): p. 799-838.

72. Xie, J., M. Ihara, Y. Jung, I.K. Kwon, S.H. Kim, Y.H. Kim, and T. Matsuda, Mechanoactive scaffold design based on microporous poly (L-lactide-co-e-caprolactone) for articular cartilage tissue engineering: Dependence of porosity on compression forceapplied mechanical behaviors. Tissue Engineering, 2006. 12(3): p. 449-458.

73. Moroni, L., J. De Wijn, and C. Van Blitterswijk, 3D fiber-deposited scaffolds for tissue engineering: influence of pores geometry and architecture on dynamic mechanical properties. Biomaterials, 2006. 27(7): p. 974-985.

74. Moutos, F.T. and F. Guilak, Composite scaffolds for cartilage tissue engineering. Biorheology, 2008. 45(3): p. 501-512.

75. Hing, K.A., Bioceramic bone graft substitutes: influence of porosity and chemistry. International Journal of Applied Ceramic Technology, 2005. 2(3): p. 184-199.

76. Lee, J.S., H.D. Cha, J.H. Shim, J.W. Jung, J.Y. Kim, and D.W. Cho, Effect of pore architecture and stacking direction on mechanical properties of solid freeform fabrication-based scaffold for bone tissue engineering. Journal of Biomedical Materials Research Part A, 2012. 100(7): p. 1846-1853.

77. Yamane, S., N. Iwasaki, Y. Kasahara, K. Harada, T. Majima, K. Monde, S.i. Nishimura, and A. Minami, Effect of pore size on in vitro cartilage formation using chitosan-based hyaluronic acid hybrid polymer fibers. Journal of Biomedical Materials Research Part A, 2007. 81(3): p. 586-593.

78. Pina, S., J.M. Oliveira, and R.L. Reis, Natural-based nanocomposites for bone tissue engineering and regenerative medicine: A review. Advanced Materials, 2015. 27(7): p. 1143-1169.

79. McGuigan, A.P., D.A. Bruzewicz, A. Glavan, M. Butte, and G.M. Whitesides, Cell encapsulation in sub-mm sized gel modules using replica molding. PLOS one, 2008. 3(5): p. e2258.

80. Gelain, F., Novel opportunities and challenges offered by nanobiomaterials in tissue engineering. International Journal of Nanomedicine, 2008. 3(4): p. 415.

81. Cukierman, E., R. Pankov, D.R. Stevens, and K.M. Yamada, Taking cell-matrix adhesions to the third dimension. Science, 2001. 294(5547): p. 1708-1712.

82. Geckil, H., F. Xu, X. Zhang, S. Moon, and U. Demirci, Engineering hydrogels as extracellular matrix mimics. Nanomedicine, 2010. 5(3): p. 469-484. 
83. Madihally, S.V. and H.W. Matthew, Porous chitosan scaffolds for tissue engineering. Biomaterials, 1999. 20(12): p. 1133-1142.

84. Ho, M.-H., P.-Y. Kuo, H.-J. Hsieh, T.-Y. Hsien, L.-T. Hou, J.-Y. Lai, and D.-M. Wang, Preparation of porous scaffolds by using freeze-extraction and freeze-gelation methods. Biomaterials, 2004. 25(1): p. 129-138.

85. Ma, J., H. Wang, B. He, and J. Chen, A preliminary in vitro study on the fabrication and tissue engineering applications of a novel chitosan bilayer material as a scaffold of human neofetal dermal fibroblasts. Biomaterials, 2001. 22(4): p. 331-336.

86. Ju, Y.M., K. Park, J.S. Son, J.J. Kim, J.W. Rhie, and D.K. Han, Beneficial effect of hydrophilized porous polymer scaffolds in tissue-engineered cartilage formation. Journal of Biomedical Materials Research Part B: Applied Biomaterials, 2008. 85(1): p. 252-260.

87. Nam, Y.S., J.J. Yoon, and T.G. Park, A novel fabrication method of macroporous biodegradable polymer scaffolds using gas foaming salt as a porogen additive. Journal of Biomedical Materials Research, 2000. 53(1): p. 1-7.

88. Hollister, S.J., Porous scaffold design for tissue engineering. Nature Materials, 2005. 4(7): p. 518-524.

89. Beşkardeş, I.G. and M. Gümüşderelioğlu, Biomimetic apatite-coated PCL scaffolds: effect of surface nanotopography on cellular functions. Journal of Bioactive and Compatible Polymers, 2009. 24(6): p. 507-524.

90. Geng, L., Y. Wong, D. Hutmacher, W. Feng, H. Loh, and J. Fuh, Rapid Prototyping of 3D Scaffolds for Tissue Engineering Using a Four-Axis Multiple-Dispenser Robotic System. 


\section{3D Printed Chitosan Hydrogel-based Scaffolds}

\subsection{Introduction}

The microenvironment surrounding the cells in an organism is a highly complex 3D network of organic macromolecules and inorganic ions of topographic significance. It is responsible for invoking a multitude of biological signals governing cell behaviour, survival, migration and growth [1]. Mimicking this microenvironment has been identified as a crucial step towards in situ tissue regeneration. Considerable research has been directed towards developing networks mimicking such microenvironments in laboratory conditions.

Rapid prototyping (RP) or solid freeform fabrication (SFF) has recently advanced towards developing biologically relevant 3D structures that are biocompatible and non-toxic for various applications such as tissue regeneration, in vitro organ development, bone fixtures and replacement materials, and anatomical models for preclinical drug screening [2-4]. Over the years, considering the increasing incidence of bone-related disease and issues with natural bone grafts (autografts, allografts and xenografts), much research has been directed towards developing artificial bone grafts via tissue engineering [5, 6]. Focussing on fabricating bone graft materials, RP allows for precise control over the internal architecture of scaffolds and the overall anatomical symmetry with the implant site. Furthermore, RP can be easily integrated with imaging techniques such as converting computed tomography (CT) images of the affected organs to printer specific files, hence allowing for personalization of the printed grafts according to individual patient disease/injury specifications $[7,8]$. This technology can precisely tailor the size, shape, interconnectivity, branching, geometry and orientation of a printed graft thereby enhancing control over its mechanical properties, biological effects and degradation kinetics.

The first technique developed in this field was stereolithography (SL) which uses lasers to photo-polymerize biocompatible liquid polymer materials. The application of SL was limited to photo-polymerizable biocompatible materials. The available number of different photo-polymerizable biomaterials with the required biocompatibility and mechanical 
stability for bone grafting applications is however low and hence limited the application of SL in developing bone graft materials. Another drawback of SL is that it offered a very low print resolution, although recently, new developments in photocurable polymers have led to micro-SL which is applicable to fabrication of for example microneedles and microcylinders on surfaces [9].

Another technique which uses laser-based photopolymerization for layer-by-layer scaffolding or additive fabrication building is selective laser sintering (SLS), wherein, powdered polymers are heated using a laser beam and further sintered to fuse the melted powder together to form a solid mass. Calcium phosphate, calcium polyphosphate, and hydroxylapatite-based porous bone grafts have been built using SLS by mixing these with photocurable polymers or monomer powders [10]. Grafts fabricated via this method were found to be biocompatible with high toughness and bending strength; resulting in the formation of mineralized bone upon implantation - an example of which was at the mandibular bone site in canines [11].

Additive fabrication without the use of lasers, known as 3D printing technology, was first developed at MIT [12]. Powdered biomaterials are 'glued' layer-by-layer using a binder material deposited by a printer nozzle over a layer of powder spread on a platform. Although desired porous microstructures can be printed layer-by-layer using this technology, postprocessing is difficult due to clogging of pores by the unbound powder. This along with the presence of organic solvents in the binders are major drawbacks when it comes to biological applications. The powder-based technology was further improved upon by extrusion-based 3D printing techniques such as fused disposition modelling (FDM), and 3D-Bioplotting.

In FDM, the extrusion of melt-based biomaterials in a layer-by-layer fashion across the Zaxis results in 3D scaffolds [13, 14]. However, FDM is limited to thermoplastics with high melt viscosity and hence does not allow for in-vitro cell printing, whereas 3D-Bioplotters, another variant of extrusion-based printers, use polymer or hydrogel-based inks. 3-D Bioplotters make it possible for the accommodation of live cells within the printing ink before printing. Furthermore, the replacement of the traditional thermal or piezoelectric print heads in a 3D-Bioplotter eliminates the requirement of heat, that could kill the cells loaded within the gel [15]. With the advent of 3D bioplotters, there has been a paradigm shift for in situ tissue regeneration from the use of synthetic implants and tissue grafts to tissue engineered scaffolds that are biodegradable. 
In this research work, we are using a custom-designed nozzle extrusion-based 3D printer to fabricate biocompatible scaffolds using chitosan biopolymer-based hydrogels as inks. Layers of the printed gel are laminated to each other using a suitable physical crosslinking agent. This agent deprotonates the hydrogel chains rendering them slightly insoluble in water, enabling the layers to adhere to each other during printing and thus building the final 3D structure. The first report on 3D printed chitosan hydrogel-based scaffolds was in 2002, which indicated good in vitro biocompatibility and enhanced osteoblast cell proliferation in a symmetric lattice-based chitosan and chitosan-hydroxyapatite composite scaffolds [16, 17]. Later Geng et al. demonstrated the feasibility of using chitosan hydrogel-based inks to print cranial patches based on CT scan images that were converted to printer compatible models using a dual extrusion robotic printer [7]. Furthermore, Lim et al. experimented with the application of cryogenic rapid prototyping by using a cold printing platform to freeze printed structures during printing in order to develop chitosan hydrogel-based scaffolds with controlled micro architecture (pore size and pore orientation) and found that the scaffold architecture affected both cellular infiltration and neo-vascularization [18]. Later, Lee et al. reported that such scaffolds however did not exhibit the minimal mechanical strength requirement to support bone regeneration in vivo and hence cannot be used as artificial bone grafts but can be considered for soft tissue regeneration [19]. More recently, complex composite 3D printed scaffolds based on chitosan-collagen-tricalcium phosphate-based hydrogels were reported which when seeded with osteoblast-like cells and implanted at defect sites in sheep showed high rates of new bone formation at the defect sites [20].

The McGrath research group has been working with chitosan hydrogel-based templates over the past several years with the aim of developing composites with nanostructures comparable to those that characterise nacre on a laboratory scale. Calcium carbonate crystallites with a morphology similar to that of nacre in its early stages of development can be developed in a 2D chitosan hydrogel-based template using the, herein called, McGrath mineralization method (discussed in detail in Chapter 1 and 3) [21]. Since nacre in its natural form has a mechanical strength comparable to that of natural bone, this work has now become the basis for developing 3D chitosan hydrogel-based composite structures that have mechanical strength comparable to that of natural bone [22].

Overall, this research investigates the fabrication of replicable 3D chitosan hydrogel-based composite structures that can support bone formation in vivo mechanically and also have the necessary porosity attributes to facilitate nutrient exchange, cell mobility and 
transportation. The first step in achieving this is exploring the possibility of fabricating stable chitosan hydrogel-based 3D scaffolds using a custom-designed nozzle extrusionbased 3D printer. The structure of the scaffold was chosen with the focus of fabricating high strength materials without compromising on the total porosity of the final structure.

In this Chapter,

(a) the transition from 2D chitosan hydrogel-based films to 3D chitosan hydrogel-based scaffolds is explained;

(b) the technique adopted to fabricate stable 3D scaffolds using our custom-designed 3D printer with chitosan hydrogel as the printing ink is described;

(c) the effect of various drying techniques, such as air-drying, freeze-drying and critical point-drying on the physical properties of the final printed scaffold, studied using scanning electron microscopy (SEM), cryo-SEM and X-ray micro tomography (micro-CT), from an application point of view as future bone graft material is presented; and

(d) the porosity of the final air-dried scaffold is estimated along with an investigation of their swelling behavior in phosphate buffered saline $(\mathrm{pH} 7.2)$. 


\subsection{Materials and methods}

Stable 3D chitosan hydrogel-based scaffolds with different macropore dimensions were fabricated via 3D printing. These scaffolds were characterized both in the wet, using cryoscanning electron microscopy (cryo-SEM) and in the dried state, using scanning electron microscopy (SEM) and micro-CT. Two macropore sizes were used in order to identify if the macropores have an effect on (1) the resultant extent of mineralization when the scaffolds are subjected to the McGrath mineralization technique (discussed in Chapter 3) and (2) the mechanical properties of the scaffold or the final composites (discussed Chapter 5). Data reported in the literature indicate that pore sizes $>350 \mu \mathrm{m}$ are suitable for bone tissue regeneration and that both the porosity and the pore size may have an effect on the mechanical strength of a given structure [23, 24]. Hence, an optimal balance between the chosen pore size and the resultant porosity is required for the best mechanical and biological performance of the final material.

\subsubsection{Materials}

Analytical grade chitosan (MW: 100,000-300,000 g/mol) was purchased from Pure Science Ltd. Acetic acid (Univar, AR grade) and sodium hydroxide (Panreac) were used as received. All solutions used in this study were prepared using water purified using a Sartorius Arium 611UV purification system (18.2 $\mathrm{M} \Omega \mathrm{cm}$ resistivity).

1X phosphate buffered saline (PBS) was prepared by dissolving $8 \mathrm{~g} \mathrm{NaCl}$ (Pure Science Ltd), $0.2 \mathrm{~g} \mathrm{KCl}$ (Sigma Aldrich), $0.24 \mathrm{~g} \mathrm{KH}_{2} \mathrm{PO}_{4}$ (Mallinckrodt Barker Inc.) in $800 \mathrm{~mL}$ of purified water. After $\mathrm{pH}$ adjustment to 7.4 with $\mathrm{HCl}$, a further $200 \mathrm{~mL}$ of purified water was added make the final volume of 1 L $1 X$ PBS.

\subsubsection{Rheological characterization}

\section{Chitosan hydrogel preparation}

Chitosan hydrogel was used as the printing ink. Printing inks with varying concentration of chitosan were prepared by dissolving $2 \% \mathrm{w} / \mathrm{v}$ chitosan $(2 \mathrm{~g}$ in $100 \mathrm{~mL}$ of purified water $)$ in $1 \% \mathrm{v} / \mathrm{v}$ acetic acid ( $1 \mathrm{~mL}$ in $100 \mathrm{~mL}$ of chitosan solution) $(\mathrm{pH} \mathrm{3.0)}$ and 5\% w/v chitosan in $2 \%$ acetic acid ( $\mathrm{pH} 2.8$ ). The carbohydrate/water mixture was continuously stirred for $6 \mathrm{~h}$ at $700 \mathrm{rpm}$ followed by centrifugation at $4000 \mathrm{rpm}$ for $5 \mathrm{~min}$ to obtain a homogenous hydrogel. 
The rheological properties of the chitosan hydrogel-based inks were studied using an AR2000 rheometer (TA Instruments) with a cone-plate geometry (60 mm diameter, $0 \mathrm{~min}$ $37 \mathrm{~s}$ cone angle, and $63 \mu \mathrm{m}$ gap). The variation in the viscosity of chitosan hydrogels prepared by dissolving different amounts of chitosan powder in purified water in the presence of acetic acid, with increasing shear rates was examined in stepped flow experiments under a step ramped shear rate from $0.01 \mathrm{~s}^{-1}$ to $100 \mathrm{~s}^{-1}$ at $25^{\circ} \mathrm{C}$. Each step was $30 \mathrm{~s}$ long and readings from the final $10 \mathrm{~s}$ were averaged to obtain a single presented datum point. A strain sweep was performed at a frequency of $1 \mathrm{~Hz}$ for strain percentages of $0.01 \%$ to $500 \%$ to identify the linear viscoelastic response of the hydrogels with respect to strain. A suitable strain (\%) within the linear elastic limit of the hydrogel was then chosen to study the behaviour of the hydrogel under increasing oscillatory frequency. Accordingly, the hydrogels were tested at $2 \%$ strain and subjected to oscillating frequency varying from 0.01 $-100 \mathrm{~Hz}$ and their behaviour was analysed.

To compare the rheological behaviour of the chitosan hydrogel inks under shear stress with the actual shear rate experienced by the hydrogel during the printing process, it is necessary to estimate the shear rate at the tip of the extruding nozzle. The actual shear rate experienced during extrusion in the case of Newtonian fluids is calculated based on the Hagen Poiseuille equation given below [25].

$$
\text { Shear rate } \dot{\gamma}=\frac{4 \mathrm{Q}}{\pi \mathrm{R}^{3}}
$$

However, for shear-thinning fluids such as in the case of chitosan hydrogels, this formula was modified with a Rabinowitsch correction (Eq 2), as explained in reference [26]

$$
\dot{\gamma}=\left(\frac{3 n+1}{4 n}\right) \frac{4 \mathrm{Q}}{\pi \mathrm{R}^{3}}
$$

where $n$ is the power-law index, $\mathrm{Q}$ is the volume flow rate, and $\mathrm{R}$ is the radius of the extrusion nozzle. Since a tapering nozzle is used in this research, the shear rate at the tip of the nozzle calculated is only an estimate, since variation in the nozzle dimensions along the hydrogel flow path through the syringe barrel and the nozzle was not taken into consideration. The volume flow rate $\mathrm{Q}$ can be estimated using the equation

$$
\mathrm{Q}=\frac{\pi \mathrm{d}^{2}}{4} \dot{v}
$$


where $\dot{v}$ is the velocity with which the extrusion nozzle moves and $\mathrm{d}$ is the diameter of the printed hydrogel.

\subsubsection{Scaffold fabrication}

Based on the rheological analysis (discussed in detail in section 2.3.1), chitosan hydrogel prepared by dissolving $5 \% \mathrm{w} / \mathrm{v}$ chitosan in purified water in the presence of $2 \% \mathrm{v} / \mathrm{v}$ acetic acid was chosen as the most appropriate hydrogel composition to be used as the printing ink in our custom-designed 3D printer.

\section{D printing}

The 3D printer used in this research was custom-designed based on the open-source MendelMax 2.0 by Maker's Tool Works, which uses custom-built syringe extruders and a heated fan attachment to enable printing with a variety of liquid materials including (but not limited to) pastes, liquid ceramics, hydrogels, RTV silicones and the subsequent thermosetting of these materials.

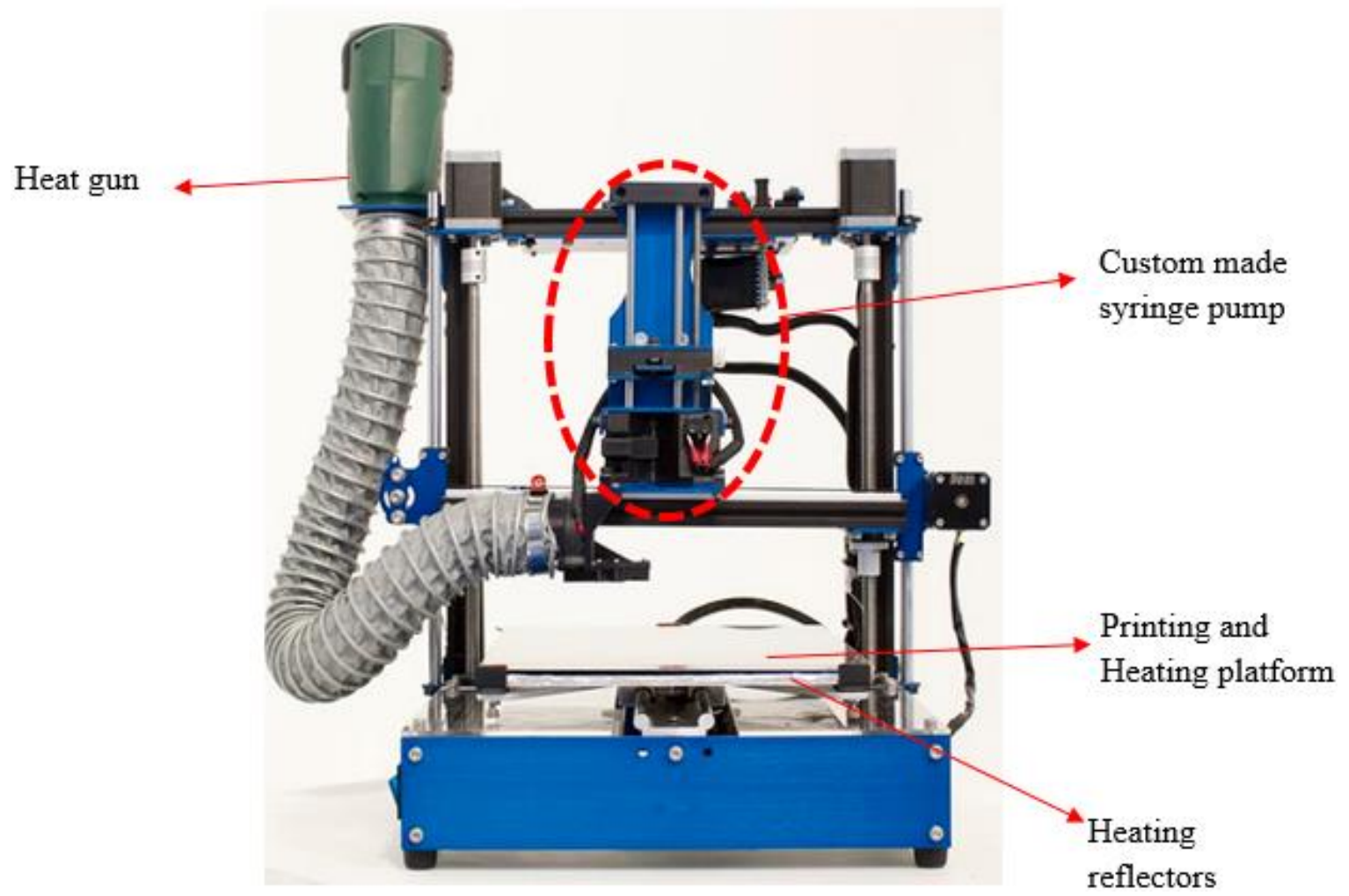

Figure 2.1: The custom-designed 3D printer used to fabricate all 3D chitosan hydrogelbased scaffolds in this research.

The 3D printer has been equipped with syringe pumps, custom-made for MendelMax 2.0, which when fitted with syringes allow for the precise dispensation of liquid materials onto 
the 3D printer's build platform. In particular, syringe pumps are paired with Luer lock syringes that facilitate the use of different needle/tip sizes to be attached according to the required resolution of the printed model. Printing was performed at room temperature at a speed of $6 \mathrm{~mm} / \mathrm{s}$ (speed of movement of printing tip) using a $5 \mathrm{~mL}$ Luer lock syringe attached with plastic nozzles of $0.42 \mathrm{~mm}$ internal diameter. The rate and pressure of extrusion/feed rate of the hydrogel are automatically controlled by the Sli3er software based on the input speed of printing and the diameter of the nozzle.

A computer-aided designing (CAD) platform was used to design the structure of the scaffold. Tinkercad, a free browser-based 3D design and modelling tool was used to design the scaffold structure which was then downloaded as a 3D printer compatible .STL file. Repetier-Host V1.6.2 software developed by Hot-World GmbH \& Co.KG, Germany enable the input of the .STL file into the printer. The CAD model developed for this research is a hollow cylinder enclosing ordered cubic lattices. The specifics of the model are shown in Figure 2.2 (a). The dimensions of the CAD model used in this study are $15 \mathrm{~mm}$ diameter $\times$ $16 \mathrm{~mm}$ height unless otherwise mentioned.

The specific CAD model used for the hydrogel systems was selected based on data obtained from uniaxial unconfined compression testing of various 3D printed symmetric lattice-based structure designs that were fabricated by 3D printing using high strength acrylonitrile butadiene styrene (ABS) thermoplastic filaments using an FDM-based 3D printer (UP Box). An Instron $1126(\max$ load $250 \mathrm{kN})$ universal testing instrument was used to perform the compression testing.

Based on the data obtained from the ABS-based structures (refer to Appendix 1), a cubic lattice-based CAD model was chosen for this work. Accordingly, cubic lattice-based CAD structures were designed by placing individual struts $1.5 \mathrm{~mm}$ or $2 \mathrm{~mm}$ (depending on the required macropore dimension in the final structure) apart within individual layers followed by $90-0-90^{\circ}$ rotation of the overlying printed layers. Scaffolds with two different macropore dimensions (as per the CAD model) were fabricated namely, $1.5 \mathrm{~mm} \times 1.5 \mathrm{~mm}$ or $2.0 \mathrm{~mm}$ $\times 2.0 \mathrm{~mm}$. The struts in the ordered lattices were $0.7 \mathrm{~mm}$ wide and $0.3 \mathrm{~mm}$ thick. A schematic representation of the experimental pathway followed is depicted in Figure 2.2 (b). The print pattern of the CAD model is rotated by $0^{\circ}-90^{\circ}$ on subsequent layers in the $\mathrm{Z}$ - direction as the print progresses. Based on the user input parameters such as the printer nozzle size $(0.42 \mathrm{~mm})$ and the speed of printing (screen shots of the print configuration are 
shown in Figure 2.3), the CAD model is sliced into individual print layers each $0.3 \mathrm{~mm}$ thick.

(a)

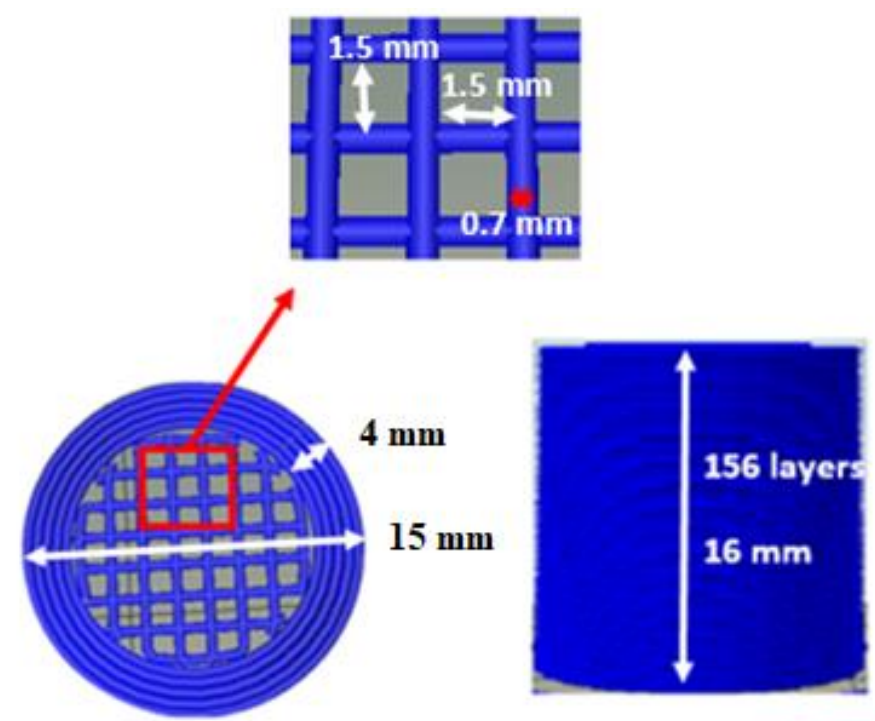

(b)

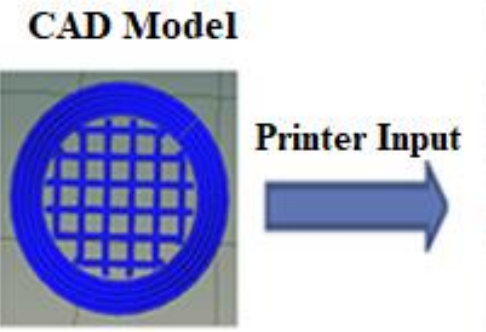

3D printing using $5 \%$ chitosan hydrogel
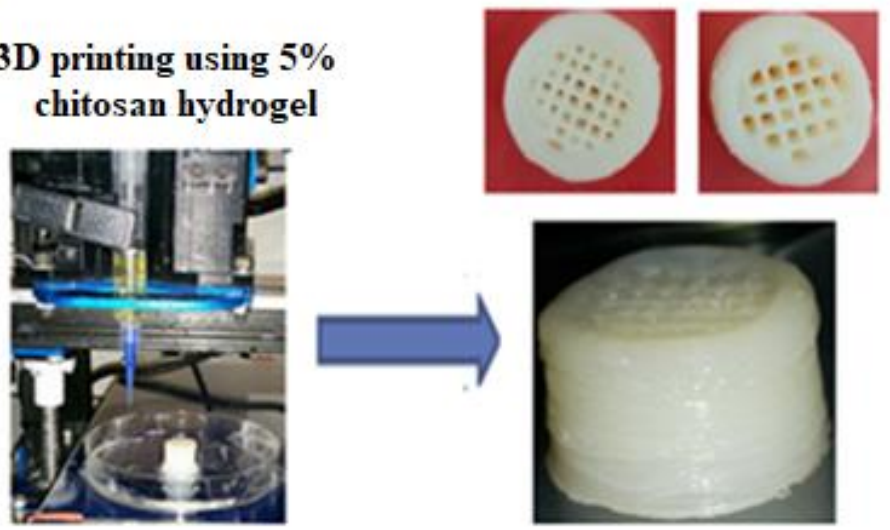

Figure 2.2: (a) The specifications of the CAD model (magnified image) input for the 3D printer and (b) a schematic representation of the experimental procedure.

In order to facilitate lamination of individual layers onto each other, the G-Code for individual prints was modified to facilitate the print nozzle being lifted to a height of $40 \mathrm{~cm}$ above the print platform (G1 Z40; allows the printer nozzle to be lifted to $40 \mathrm{~cm}$ above the print platform) coupled with the inclusion of a pause code (G4 S20; introduces a 20 s pause to the printing process), thus, facilitating any manual manipulations. Each layer is laminated to the previous layer using a $0.7-1.5 \%$ ethanolic $\mathrm{NaOH}$ solution (70:30 $\mathrm{NaOH}$ :ethanol). This is a manual process completed during the 20 s pause between the printing of each layer. The excess solution is removed using lint-free wipes. This is an essential component of the printing process because this process ensures that the individual chitosan hydrogel layers strongly adhere to each other and thus the integrity of the printed scaffold is maintained. Hence, chemical treatment is required after every layer is printed. This treatment is a 
physical crosslinking technique wherein ethanolic $\mathrm{NaOH}$, at the above-mentioned concentrations, ensures the partial deprotonation (gelation) of the hydrogel matrix such that there is strong adhesion between the already printed layer and new layer printed over it.

Once fabricated, the scaffold was further rested in $1 \mathrm{M} \mathrm{NaOH}$ solution over a period of 24 h. The scaffold was then washed thoroughly with purified water until neutral $\mathrm{pH}$ was obtained and stored in purified water at room temperature until further use. In this fabrication technique, we do not use any chemical crosslinking agents such the popular glutaraldehyde. Considering the cytotoxicity effects of such procedures on the human cell lines and also the fact that radiation-based (UV radiation) crosslinking restricts the thickness of the printed structures to a few layers, which once crosslinked, will restrict the adhesion of new layers of printed gel on to it [27-29], these methods were deemed disadvantageous.

(a)

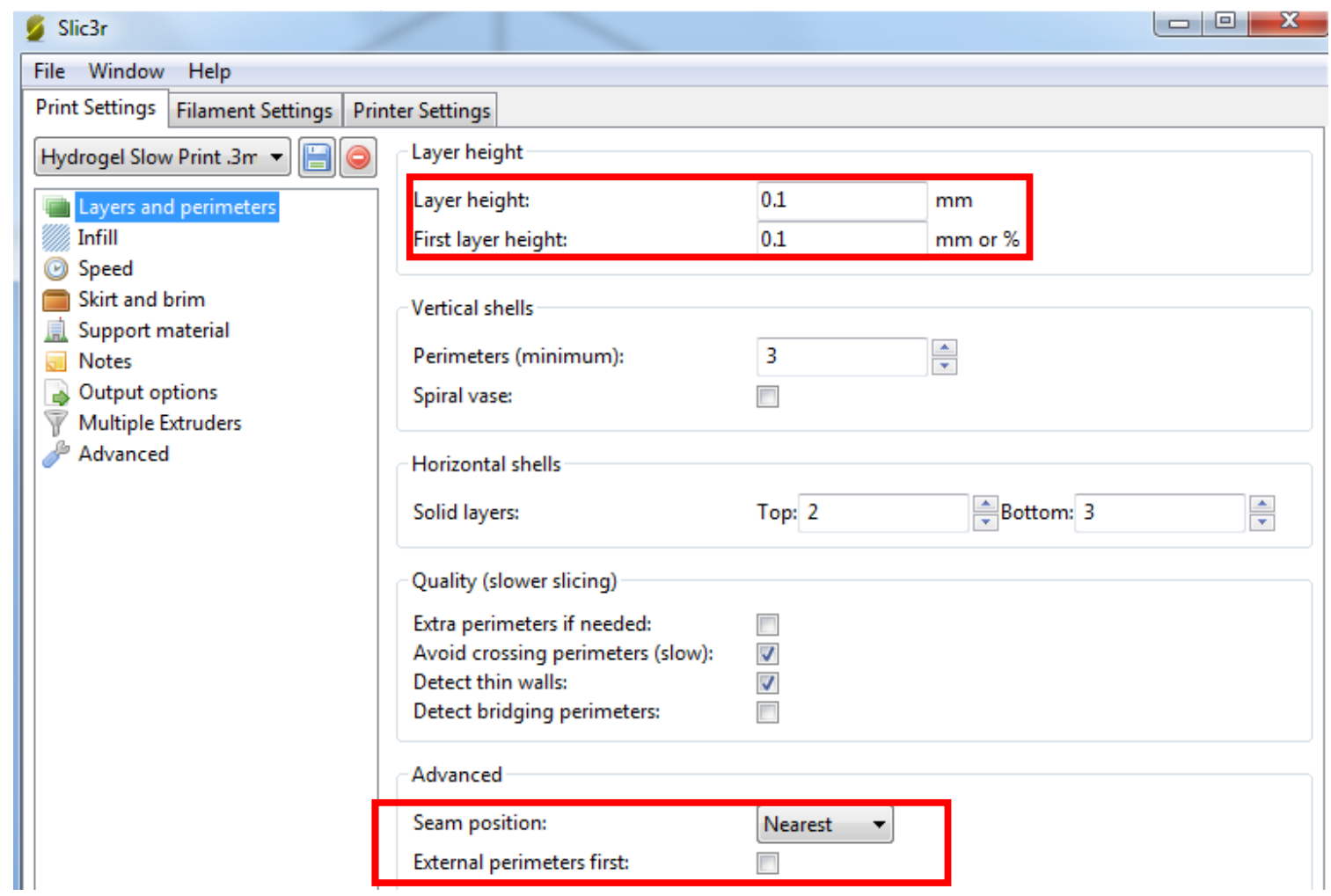


(b)

\begin{tabular}{|c|c|c|c|}
\hline \multicolumn{4}{|l|}{ File Window Help } \\
\hline \multicolumn{4}{|c|}{\begin{tabular}{|l|l|l|} 
Print Settings & Filament Settings & Printer Settings \\
\end{tabular}} \\
\hline Hydrogel Slow Print.3m $\rightarrow 0$ & IntmI & & \\
\hline \multirow{12}{*}{\begin{tabular}{|l} 
Layers and perimeters \\
Infill \\
I) Speed \\
Skirt and brim \\
Support material \\
Sotes \\
$\Rightarrow$ Output options \\
Dultiple Extruders \\
S Advanced
\end{tabular}} & Fill density: & $\sim \%$ & \\
\hline & Fill pattern: & concentric & \\
\hline & Top/bottom fill pattern: & concentric & \\
\hline & Reducing printing time & & \\
\hline & Combine infill every: & layers & \\
\hline & Only infill where needed: & 回 & \\
\hline & Advanced & & \\
\hline & Solid infill every: & layers & \\
\hline & Fill angle: & $A:$ & \\
\hline & Solid infill threshold area: & $\mathrm{mm}^{2}$ & \\
\hline & $\begin{array}{l}\text { Only retract when crossing } \\
\text { perimeters: }\end{array}$ & $\nabla$ & \\
\hline & Infill before perimeters: & $\square$ & \\
\hline
\end{tabular}

(c)

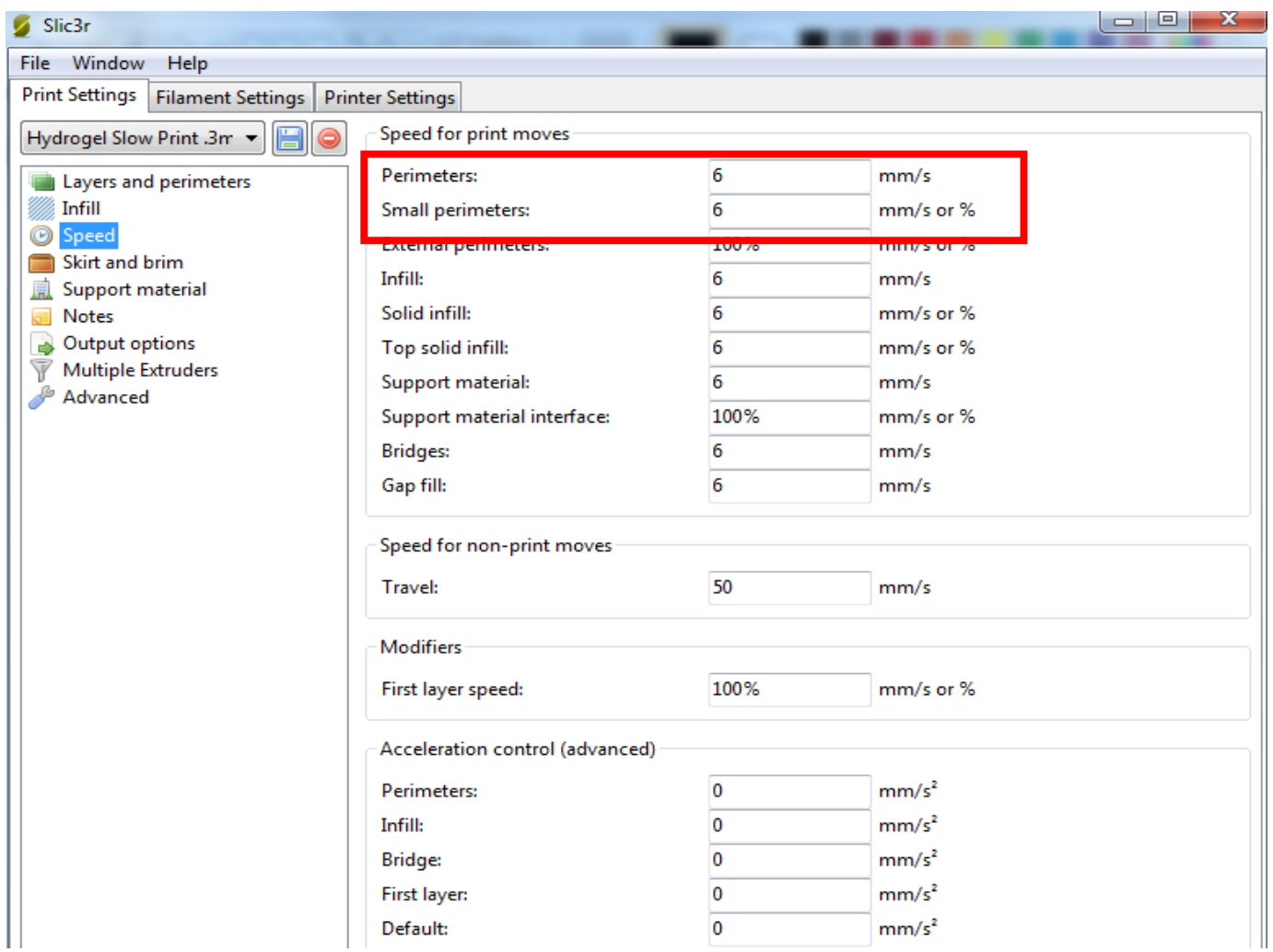

Figure 2.3: Screen shots of print parameters as configured in the slic3r software defining the (a) speed of printing, layer height, and (b) infill pattern; and (c) speed of printing when printing various parameters (highlighted in red boxes in each image). No support material was used in this study and the values defining the same are irrelevant. 


\subsubsection{Scaffold drying}

A number of different drying treatments were applied to the as-printed, non-mineralized chitosan hydrogel-based scaffolds.

(a) Air-drying: The scaffolds were dried under laminar air flow over a period of $24 \mathrm{~h}$. The orientation of the scaffolds was changed approximately hourly during the drying period in order to promote uniform drying without inducing any geometric disfiguration in the scaffolds caused by the surface tension effects of water evaporating from the hydrogel.

(b) Freeze-drying: The wet chitosan hydrogel-based scaffolds were pat-dried to remove any excess water on the surface and within the macropores and then pre-frozen in liquid nitrogen. The scaffolds were then subjected to freeze-drying/lyophilisation using a VirTis Sentry freeze drier for a period of $24 \mathrm{~h}$.

(c) Critical point drying: This technique is based on drying a wet specimen at the critical point (temperature and pressure) of the solvent to be removed from the specimen. At the critical point, the solvent can be evaporated without causing any disruption to the structure of the specimen that may arise due to the surface tension of this solvent. Water has a critical point of $374{ }^{\circ} \mathrm{C}$ and 3212 p.s.i. However, under such conditions, sensitive specimens would decompose. Liquid $\mathrm{CO}_{2}$ has a critical point of $31{ }^{\circ} \mathrm{C}$ and 1072 p.s.i and hence, strategies to replace water with liquid $\mathrm{CO}_{2}$ have been identified. This was achieved by equilibrating the scaffold successively in ethanol solutions of increasing ethanol concentration: $30 \%$ up to $95 \%$ each for 10 min and finally in $100 \%$ ethanol/absolute ethanol thrice for 20 min each. Ethanol was then replaced with acetone by equilibrating the scaffold in acetone thrice for 20 min each. Acetone is easily replaced by liquid $\mathrm{CO}_{2}$ during the drying process in the critical point dryer. The samples where dried once using a Baltec CPD 030 critical point dryer.

\subsubsection{Swelling behaviour of chitosan hydrogel-based scaffolds}

To study the swelling behaviour of the scaffold, non-mineralized scaffolds dried via airdrying, freeze-drying and critical point-drying were rehydrated by soaking in PBS (pH 7.4) for 1 week (solution was not changed during the soaking period). The scaffolds were weighed in the dried and rehydrated states and the solvent uptake ratio and percentage volume change of the dried scaffolds due to the uptake of PBS were estimated as follows: 


$$
\begin{gathered}
\text { Solvent uptake ratio }=\frac{M_{w}}{M_{d}} \\
\% \text { Volume change }=\frac{\left(V_{w}-V_{w} V_{d}\right)}{V_{d}} \times 100 \%
\end{gathered}
$$

where $M_{d}, M_{w}, V_{w}$ and $V_{d}$ are the mass of the scaffold in the dried and rehydrated states and the volume of the scaffolds in the rehydrated and dried states respectively [30].

The changes in the volume of the scaffold can also be directly estimated based on the dimension of the swollen scaffold. All values are expressed as the average \pm mean standard deviation of the values obtained for three sets of samples.

\subsubsection{Scanning electron microscopy (SEM)}

SEM was performed to investigate the structural features of the dried chitosan hydrogelbased scaffolds. This technique allows analysis of the surface up to a depth of $\sim 1 \mu \mathrm{m}$. For SEM, dried scaffolds were mounted on aluminium stubs using double-sided carbon tape. The samples were then coated with $30 \mathrm{~nm}$ of platinum using a JFC-1500 ion sputtering device. Images were acquired using a JEOL JSM-6610LV field emission SEM in secondary electron detector (SEI) mode. An accelerating voltage of $20 \mathrm{kV}$ was typically used.

Cryo-SEM was used to analyse the extensive porous network within wet 3D scaffolds. Images were acquired on a JEOL 6500F SEM equipped with a cryo-stage. An accelerating voltage of $3 \mathrm{kV}$ and a probe current of $13 \mu \mathrm{A}$ were used. Samples were frozen using a jet freezing system, wherein, a small piece of the wet scaffold was sandwiched between two $\mathrm{Cu}$ holders. The copper disc was mounted onto a sample holder and transferred to the jet freezing chamber with a constant flow of propane gas. After pressure freezing the sample, it was dropped into a propane/liquid nitrogen mix and then transferred into the cryo-chamber within a cooled $-120^{\circ} \mathrm{C}$ high vacuum environment. The sample was then sublimated for 15 min at $-90{ }^{\circ} \mathrm{C}$ in order to expose the internal features of the sample. After sublimation, the sample was twice coated with platinum and transferred to the microscope viewing platform. The temperature of the microscope chamber was stabilized at $-120{ }^{\circ} \mathrm{C}$ while imaging. Imaging was performed in SEI mode. 
Cryo-SEM was used to image rehydrated chitosan hydrogel-based scaffolds after soaking air-dried chitosan hydrogel-based scaffolds in 1X PBS for 1 week. Samples were prepared by simply pre-freezing in liquid nitrogen instead of using the jet freezing system and before transferring into the cryo-chamber within a cooled $-120{ }^{\circ} \mathrm{C}$ high vacuum environment. The analysis was performed at an accelerating voltage of $5 \mathrm{kV}$ and probe current of $4 \mu \mathrm{A}$. For comparability, air-dried chitosan hydrogel-based scaffolds (before rehydration) were also prepared under the same conditions and imaged at a voltage of $3 \mathrm{kV}$ and probe current of 2 $\mu \mathrm{A}$.

\subsubsection{Macroporosity estimation}

The porosity of the as-designed CAD models was estimated as follows

$$
\% \text { Porosity }=\left(1-\left(\frac{\mathrm{v}_{\text {solid }}}{\mathrm{v}_{\text {scaffold }}}\right)\right) \times 100 \%
$$

where $V_{\text {solid }}$ is the solid volume and $V_{\text {scaffold }}$ is the total volume of the scaffold $\left(\pi r^{2} h\right.$, where $\mathrm{r}$ is radius and $\mathrm{h}$ is the height of the structure) [31].

\subsubsection{Micro-computed tomography (Micro-CT) analysis}

Micro-CT analysis was performed allowing 3D visualization of the internal structure of the materials. This technique allowed the porosity of air-dried 3D chitosan hydrogel-based scaffolds with different macropore dimensions to be estimated Micro-CT analysis is a nondestructive method in which the test sample, placed on a rotatory platform, is irradiated using X-rays in order to produce a 3D image of the sample. When the X-rays travel through the sample, they are attenuated and the emergent X-rays, with reduced intensities, are captured by the detector array. Based on these measurements from the detector and the Xray paths the X-ray attenuation coefficients are calculated. Based on these data, a 2D pixel map is created where each pixel is denoted by a threshold value relating to the attenuation coefficient measured under similar conditions [32].

Porosity was estimated by choosing a suitable grey scale threshold to visualize the scanned images and then the X-ray attenuation coefficient was correlated to the material density. When the sample is irradiated with X-rays, its path and the attenuation coefficients will vary with the composition or density of different regions within the test sample (chitosan polymer, pores or minerals if present); the presence of a filtering object (usually a piece of 
aluminium foil) in the X-ray path; and the energy of the X-ray used. The mean attenuation coefficient can be correlated to the material density in order to obtain a 2D map that shows the various material phases within the scaffold. Various structural features such as the pore size, pore distribution, total porosity and mineral density (in the case of composites) can be obtained. The imaging process involves rendering the 3D sample structure into a series of $2 \mathrm{D}$ sections which are reconstructed to a $3 \mathrm{D}$ image for analysis using reconstruction software.

In this study, micro-CT scanning was performed using a Bruker SkyScan1272 highresolution equipment (N.V., Kontich, Belgium) working with a source voltage of $45 \mathrm{kV}$ and a current of $180 \mu \mathrm{A}$ with an exposure time of $2800 \mathrm{~ms}$. All samples were scanned at a resolution of $2 \mu \mathrm{m}$. No filter was applied for imaging dried chitosan hydrogel-based 3D scaffolds. Raw slices were reconstructed to sequential transaxial tomograms using NRecon 1.6.9.18 reconstruction software from Bruker. Various artefacts can appear in the final image as the X-ray beam comprised of a range of photon energies passes through an object. Generally, photons with lower energies are absorbed more rapidly than high energy ones thus, resulting in high intensity regions. This is referred to as beam hardening in the image. Similarly, circular artefacts may appear in the images due to any calibration error in the detectors [33]. However, the appearance of such artefacts can be corrected using in-built algorithms that can be applied during image reconstruction. Accordingly, a beam hardening correction was applied (92\%), smoothening was set to 4 and the ring artefact correction was set to 16. Reconstructed data were further processed using CTAn 1.14.4 (Bruker microCT) analysis software. After image reconstruction, the image is segmented into regions corresponding to objects or pores by "thresholding". This creates a binary image with light (0) and dark (1) regions from grey-level ones by turning all pixels below some threshold to zero and all pixels about that threshold to one. In this analysis the threshold applied was between 17 and 255 . 


\subsection{Results and discussion}

\subsubsection{Transition from 2D chitosan hydrogel-based films to 3D scaffolds}

When using a biopolymer as the ink in an extrusion-based 3D printer, the printability and mechanical stability of the hydrogel, such that it flows under applied stress but otherwise holds its form when the stress is removed and thereby forms a stable structure, are critical properties of the hydrogel [34]. The printability is mostly determined by the viscosity of the ink and its shear-thinning behaviour as a function of time and applied extrusion pressure during printing.

Previously in the McGrath group, 2D chitosan hydrogel-based films were fabricated using hydrogels prepared by dissolving $2 \% \mathrm{w} / \mathrm{v}$ chitosan in purified water in the presence of $1 \%$ $\mathrm{v} / \mathrm{v}$ acetic acid. The viscosity of the resultant hydrogel is $0.68 \pm 0.02 \mathrm{~Pa}$.s at $0.1 \mathrm{~s}^{-1}$ shear rate. This hydrogel, when used as the printing ink in the custom-designed 3D printer to fabricate 3D scaffolds using an extrusion nozzle with an internal diameter of $0.42 \mathrm{~mm}$, it was observed that the viscosity of the gel was too low to be extruded as a continuous strand and so the CAD structure could not be printed accurately. Accordingly, in order to increase the viscosity of the hydrogel such that it could be printed as a continuous strand through a $0.42 \mathrm{~mm}$ nozzle, the concentration of the chitosan hydrogel was increased. A range of concentrations up to $5 \% \mathrm{w} / \mathrm{v}$ of chitosan were explored. It was observed that dissolving higher concentrations of chitosan in the presence of $1 \% \mathrm{v} / \mathrm{v}$ acetic acid resulted in very viscous gels. Thus, the concentration of acetic acid was also increased to $2 \% \mathrm{v} / \mathrm{v}$ upon increasing the concentration of chitosan to allow the hydrogel viscosity to be appropriately adjusted for printing. The viscosity of the resultant hydrogel prepared using $5 \% \mathrm{w} / \mathrm{v}$ chitosan dissolved in the presence of $2 \% \mathrm{v} / \mathrm{v}$ acetic acid is $86.1 \pm 3.4 \mathrm{~Pa} . \mathrm{s}$ at $0.1 \mathrm{~s}^{-1}$ shear rate. The variation in the viscosity of the two limiting chitosan hydrogels $(2 \% \mathrm{w} / \mathrm{v}$ and $5 \% \mathrm{w} / \mathrm{v}$ chitosan) as a function of the shear rate is shown in Figure 2.4.

Shear-thinning behaviour, meaning that there is a decrease in the viscosity of a viscoelastic material with an increase in the shear rate, was observed in the case of both the hydrogel formulations. Shear thinning occurs because the applied shear energy tends to align anisotropic molecules and untangle polymeric chains or large aggregates hence reducing the hydrodynamic drag, thus reducing the dissipation of energy and increasing the tendency to flow [35]. In general chitosan hydrogels exhibited shear-thinning behaviour at low shear rates at $25{ }^{\circ} \mathrm{C}$ with the behaviour being more prominent in the case of higher concentration 
hydrogels in similarity to the data reported here [36]. In the case of the low concentration chitosan hydrogel solution ( $2 \% \mathrm{w} / \mathrm{v}$ in $1 \% \mathrm{v} / \mathrm{v}$ acetic acid solution), shear thinning was observed at slightly higher shear rates than in the case of the higher concentration chitosan hydrogel (5\% w/v in $2 \% \mathrm{v} / \mathrm{v}$ acetic acid), also there was significantly less variation in the viscosity of the hydrogel at lower shear rates, with the hydrogel essentially behaving like a Newtonian fluid.

Various trail 3D printings showed that the most suitable printing ink that can be extruded as a continuous strand was $5 \% \mathrm{w} / \mathrm{v}$ chitosan hydrogel in $2 \% \mathrm{v} / \mathrm{v}$ of acetic acid solution. The estimated shear rate (based on Eq (2.1) - (2.3)) at the tip of the $0.42 \mathrm{~mm}$ internal diameter nozzle at $6 \mathrm{~mm} \mathrm{~s}^{-1}$ was approximated to be within the range of $72 \pm 7 \mathrm{~s}^{-1}$. From Figure 2.4 it is evident that the viscosity of the $2 \% \mathrm{w} / \mathrm{v}$ chitosan hydrogel at the above shear rate (marked in red) is more than an order of magnitude lower than in the case of the $5 \% \mathrm{w} / \mathrm{v}$ chitosan hydrogels and was thus unsuitable to be used as the printing ink when using a 0.42 $\mathrm{mm}$ diameter extrusion nozzle and printing speed of $6 \mathrm{~mm} \mathrm{~s}^{-1}$.

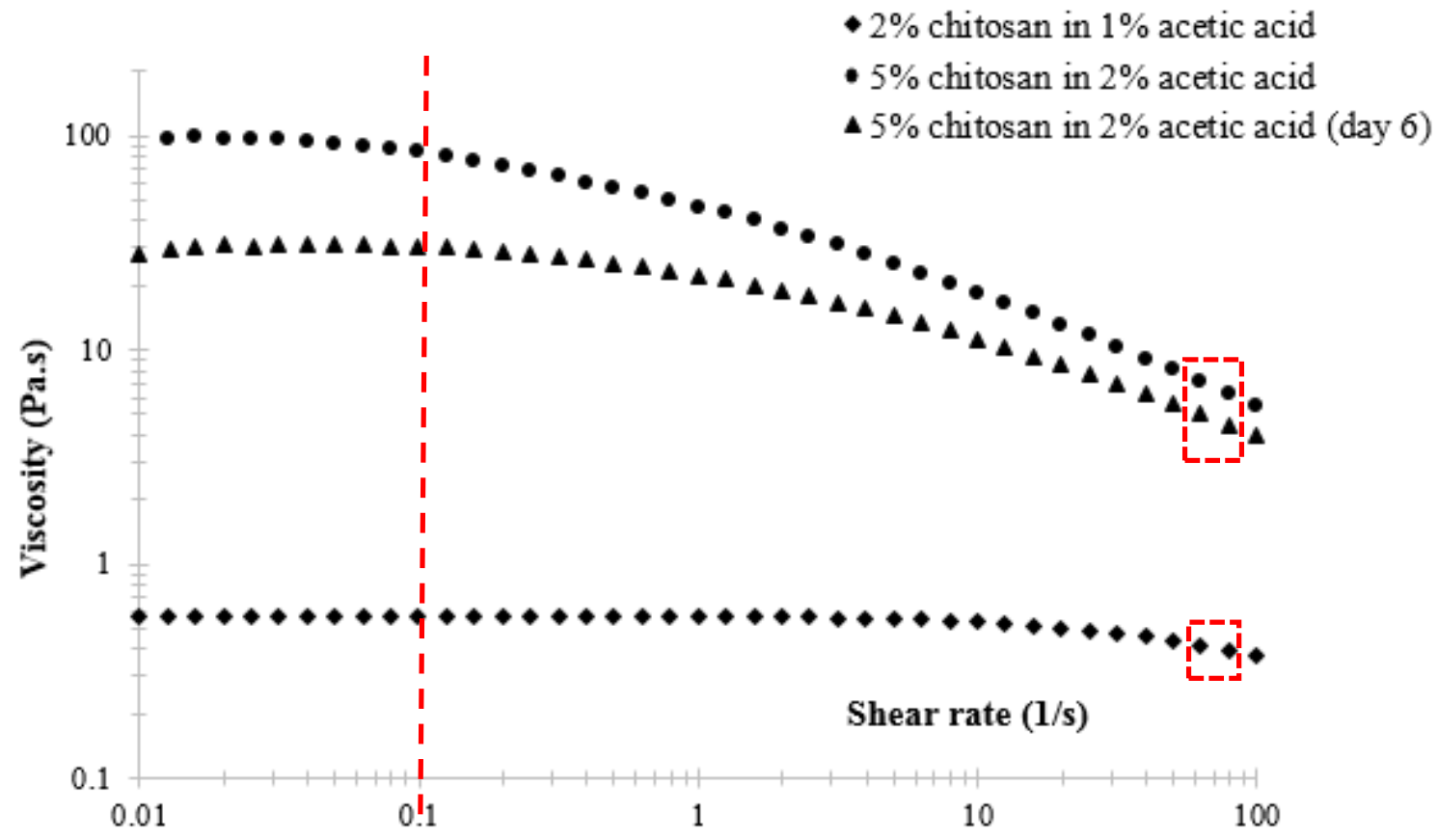

Figure 2.4: Log-log plots of the variation of viscosity of chitosan hydrogels used for fabricating $2 \mathrm{D}(2 \% \mathrm{w} / \mathrm{v}$ chitosan in $1 \% \mathrm{v} / \mathrm{v}$ acetic acid solution) and $3 D(5 \% \mathrm{w} / \mathrm{v}$ chitosan in $2 \% \mathrm{v} / \mathrm{v}$ acetic acid solution) chitosan hydrogel-based scaffolds, with increasing shear rate. The region marked with the red box represents the approximate shear rate experienced when using an extrusion nozzle of diameter $0.42 \mathrm{~mm}\left(72 \pm 7 \mathrm{~s}^{-1}\right)$ and the corresponding viscosity of the gels. The black dashed line represents $0.1 \mathrm{~s}^{-1}$ shear rate. 

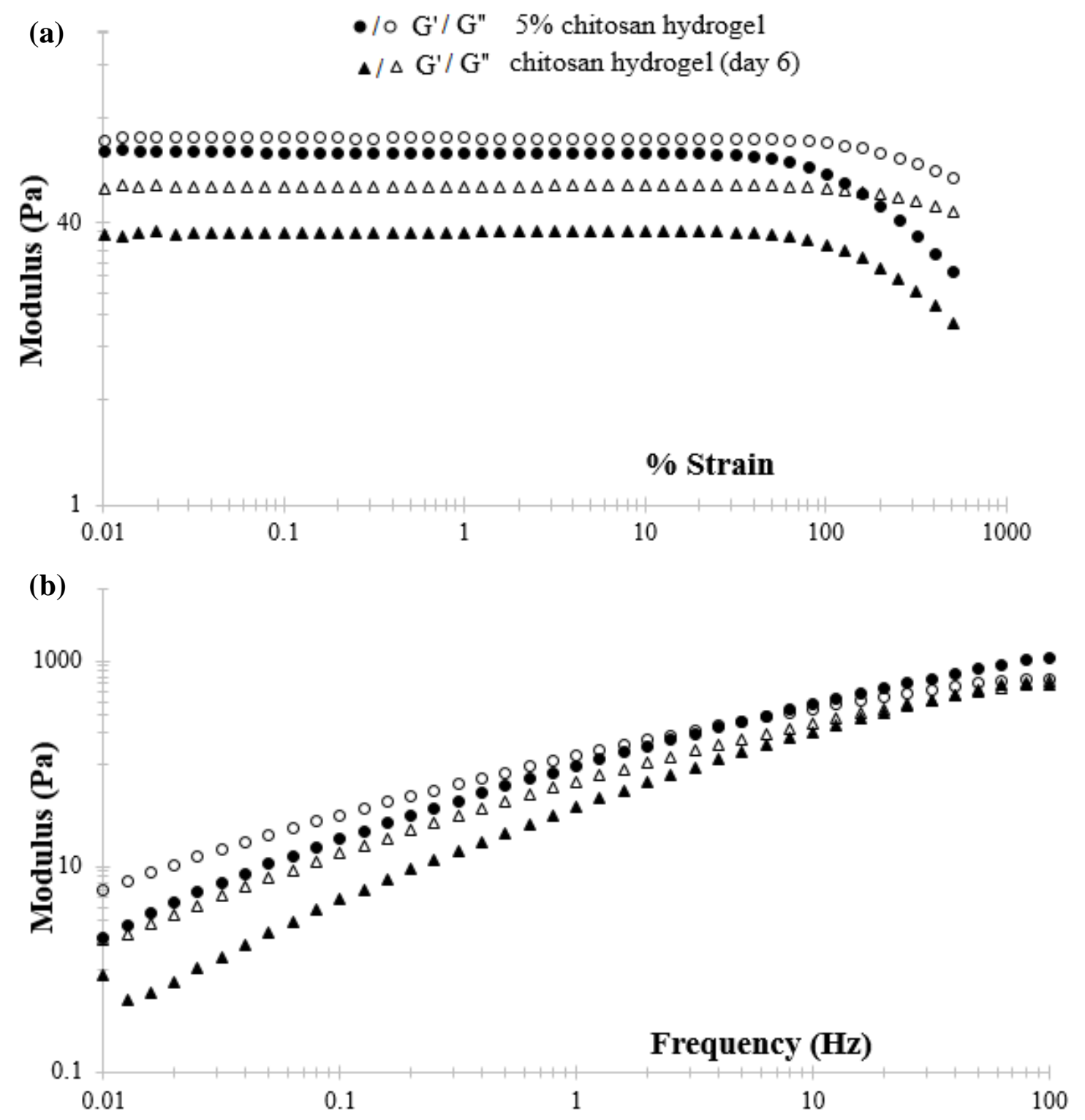

Figure 2.5: Log-log plots of (a) strain sweep, 0.01\% - 500\% at $1 \mathrm{~Hz}$ and; (b) frequency sweep $0.01-100 \mathrm{~Hz}$ at 2\% strain for freshly prepared and 6 days old 5\% w/v chitosan hydrogel prepared in the presence of $2 \% \mathrm{v} / \mathrm{v}$ acetic acid.

It was observed that the viscosity of $5 \% \mathrm{w} / \mathrm{v}$ chitosan hydrogel in $2 \%$ acetic acid decreased with time, see Figure 2.4. This can be attributed to the slow dissolution via protonation of the amine groups of the chitosan polymer chain in the presence of acetic acid. Based on the rheology studies $5 \% \mathrm{w} / \mathrm{v}$ chitosan hydrogel can be used as the printing ink for up to 6 days after gel preparation as the viscosity of the gel during the printing process is comparable at the shear rate experienced when it is extruded through a $0.42 \mathrm{~mm}$ nozzle. However, practically it was found that good prints were obtained when using the gel within 3 days of 
preparation. Based on these results, for all further studies, $5 \% \mathrm{w} / \mathrm{v}$ chitosan hydrogel (in $2 \%$ $\mathrm{v} / \mathrm{v}$ acetic acid) was used as the printing ink.

Based on the data from the strain sweep performed at $1 \mathrm{~Hz}$ for $0.01 \%-500 \%$ strain (Figure 2.5 (a)), at $2 \%$ strain, which is within the linear limit of the hydrogel, was selected to perform the frequency sweep. Figure 2.5 (b) shows the log-log plot of the variation in dynamic storage $\left(\mathrm{G}^{\prime}\right)$ and loss moduli $\left(\mathrm{G}^{\prime \prime}\right)$ for $5 \% \mathrm{w} / \mathrm{v}$ chitosan hydrogels (freshly prepared and 6 days old sample) with increasing oscillating frequency. The data at low frequencies depict the behaviour of the samples at very slow variation in the applied oscillatory stress and the opposite behaviour is depicted at higher frequencies. Similar to the behaviour of uncrosslinked polymers, $\mathrm{G}^{\prime}<\mathrm{G}^{\prime \prime}$ at lower frequencies showing predominantly viscous behaviour and then at higher frequencies $\mathrm{G}^{\prime}>\mathrm{G}^{\prime \prime}$ showing elastic behaviour. The crossover point where $\mathrm{G}^{\prime}=\mathrm{G}^{\prime \prime}$ varied as the viscosity of $5 \% \mathrm{w} / \mathrm{v}$ chitosan hydrogel decreased with time. In the case of freshly prepared hydrogels the crossover occurred at approximately 6.3 $\mathrm{Hz}$ and in the case of 6 days old sample, with lower viscosity, the cross over frequency was approximately $50 \mathrm{~Hz}$. Based on a comparison of the printability of both of these gels it can be summarized that gels with lower crossover frequencies (nearby $6 \mathrm{~Hz}$ ) are a better choice for extrusion-based printing under otherwise similar conditions.

\subsubsection{D printing}

Symmetric cylindrical lattice-based 3D chitosan hydrogel-based scaffolds based on the CAD model described in section 2.2.3 were 3D printed using $5 \% \mathrm{w} / \mathrm{v}$ chitosan hydrogel as the printing ink.

Initially, different 3D porous structures were fabricated using ABS, and their mechanical performance was assessed via uniaxial compression testing. The structures that showed the best mechanical resistance to fracture under compression were then used as the basic CAD models for 3D printing when using chitosan hydrogel as the ink.

Different porous cylindrical structures were designed whereby the pore structure was generated via repetitive cubic or hexagonal lattices as standalone structures or supported by an outer cylindrical sheath. Standalone hollow cylinders with different wall thickness, where the central column acts as a long tubular pore were also fabricated. This initial work was performed using $\mathrm{ABS}$ due to the considerable time and effort that would have been required to generate all the different structures reliably and reproducibly with the size 
specifications noted according to the ASTM D695-10 compression testing standard if chitosan hydrogel was used as the printing ink. It was assumed that since the property tested (porosity dependent compressive strength and yield strength) depends predominantly on the internal architecture of the structure (made of the same material), the trend obtained for ABS printed structures would reflect those that would be obtained for the chitosan hydrogelbased equivalents $[37,38]$. Hence this was a more expedient way of determining which structure resulted in the highest compressive strength while including high porosity and therefore which structure would be selected for use in all the other experiments

Analysis of the compression testing data revealed that the mechanical stability of hollow cylinders decreases with increasing wall thickness (images, specifications and compression testing data are given in Appendix 1). It was also observed that cubic lattices (0-90- $0^{\circ}$ rotation of the overlying printed layers) offered better resistance to complete fracture compared to hexagonal lattices (0-60-120 $0^{\circ}$ rotation of overlying layers). This could be due to the fact that in the case of the repetitive cubic lattices there is a regular overlap of struts at the nodes (points where fibres overlap) at every $3^{\text {rd }}$ layer, whereas in the case of hexagonal lattices regular overlap repeats on every $4^{\text {th }}$ layer. This can result in different deformation mechanisms under compression. Li et al. explains that the variation in the unit cell architecture of repetitive lattice-based structures can cause either bending or buckling deformation under compression which in turn affects the resultant modulus, compressive strength and plasticity of the entire structure. Cubic lattices were found to show buckling deformation which resulted in a higher modulus, compressive strength compared to rhombic dodecahedron lattices which showed bending deformation at the nodes [39].

A hexagonal lattice is comparable to a rhombic dodecahedron lattice in terms of the fibre laydown pattern and hence most likely corresponds to bending deformation at the nodes. Buckling deformation causes better fracture resistance compared to those occurring in hexagonal lattices. Also, hollow cylinders with thinner walls showed a higher resistance to buckling fracture compared to thick-walled hollow cylinders even though the latter had a higher mass. This could be attributed to the higher second moment of area of thinner-walled hollow cylinders, resulting in a higher force required to initiate buckling fracture in the cylinder [40].

Based on this data, it was deduced that a structure with a hollow cylinder enclosing ordered cubic lattices may provide a higher resistance to fracture compared to the models based on 
hollow cylinders and symmetric cubic lattice structures individually. This assumption was confirmed by the data obtained for ABS-based 3D printed structures based on a combination of hollow cylinders and cubic lattices as shown in Appendix 1. Also, this CAD model fulfilled the porosity requirement (hollow cylinder exterior being less porous compared to lattices in the middle) similar to that of bone (variation in the porosity of cortical and trabecular bone). Based on this initial work using ABS filament-based 3D printed structures, the CAD model depicted in Figure 2.2 (a) was used as the sole model for all further research. The thickness of the hollow cylinder was chosen as $4 \mathrm{~mm}$ such that a stable scaffold could be fabricated using hydrogels as the ink. This architecture was also chosen over the traditional lattice-based structures reported in the literature as an added improvement to the mechanical strength without heavily compromising on the porosity of the final composite scaffold keeping in mind that there is also a structural contribution to the mechanical properties of a material along with the contribution from its composition [41].

Using this CAD model and 5\% w/v chitosan hydrogel as the printing ink in the customdesigned 3D printer, the minimum achievable macropore dimensions in the final scaffold, (when a $0.42 \mathrm{~mm}$ diameter extrusion nozzle was used) are about $1.5 \mathrm{~mm} \times 1.5 \mathrm{~mm}$. Accordingly, upon 3D printing a $16 \mathrm{~mm}$ high and $15 \mathrm{~mm}$ diameter cylindrical CAD model with $1.5 \mathrm{~mm} \times 1.5 \mathrm{~mm}$ macropores, the resultant hydrogel scaffold is a 156 layered structure. Each layer was laminated to overlying and underlying layers with appreciable adhesion, such that although the individual layers can be peeled apart (in the wet state) with minimal force, the layers do not slide over each other without an external sheer force. The adhesion is further improved as the scaffold undergoes gelation in $1 \mathrm{M} \mathrm{NaOH}$ solution. The final scaffolds are opaque white. Scaffolds with $2.0 \mathrm{~mm} \times 2.0 \mathrm{~mm}$ macropores were also fabricated using the same extrusion nozzle in order to explore the effect of macroporosity of the scaffolds on the degree of mineralization and hence the resulting mechanical strength of the scaffolds (see Chapter 3 and 5).

\subsubsection{Effect of drying conditions on scaffold characteristics}

Cryo-SEM was used to visualise the scaffold structure in the wet state and standard SEM was used to study dried scaffolds. The electron microscopy images revealed that the structural integrity of the scaffolds was not maintained under different drying conditions. This resulted in variations in the extent of shrinkage and hence the porosity of the final dried scaffold. These variations further affect the mechanical properties of the dried scaffolds. 
Air-drying was the first drying technique tested. Figure 2.5 is a representative image of a chitosan hydrogel-based scaffold before and after air-drying. It can be observed that the scaffolds undergo considerable shrinkage during the drying process. An average shrinkage of $56 \pm 3 \%$ and $75 \pm 4 \%$ was observed with respect to the diameter and the height respectively for air-dried scaffolds after air-drying. The observed shrinkage could be due to the fact that unlike chemically crosslinked hydrogels, physically crosslinked hydrogel-based scaffolds undergo considerable shrinkage due to the surface tension effect of the evaporating water when subject to air dying.

(a)

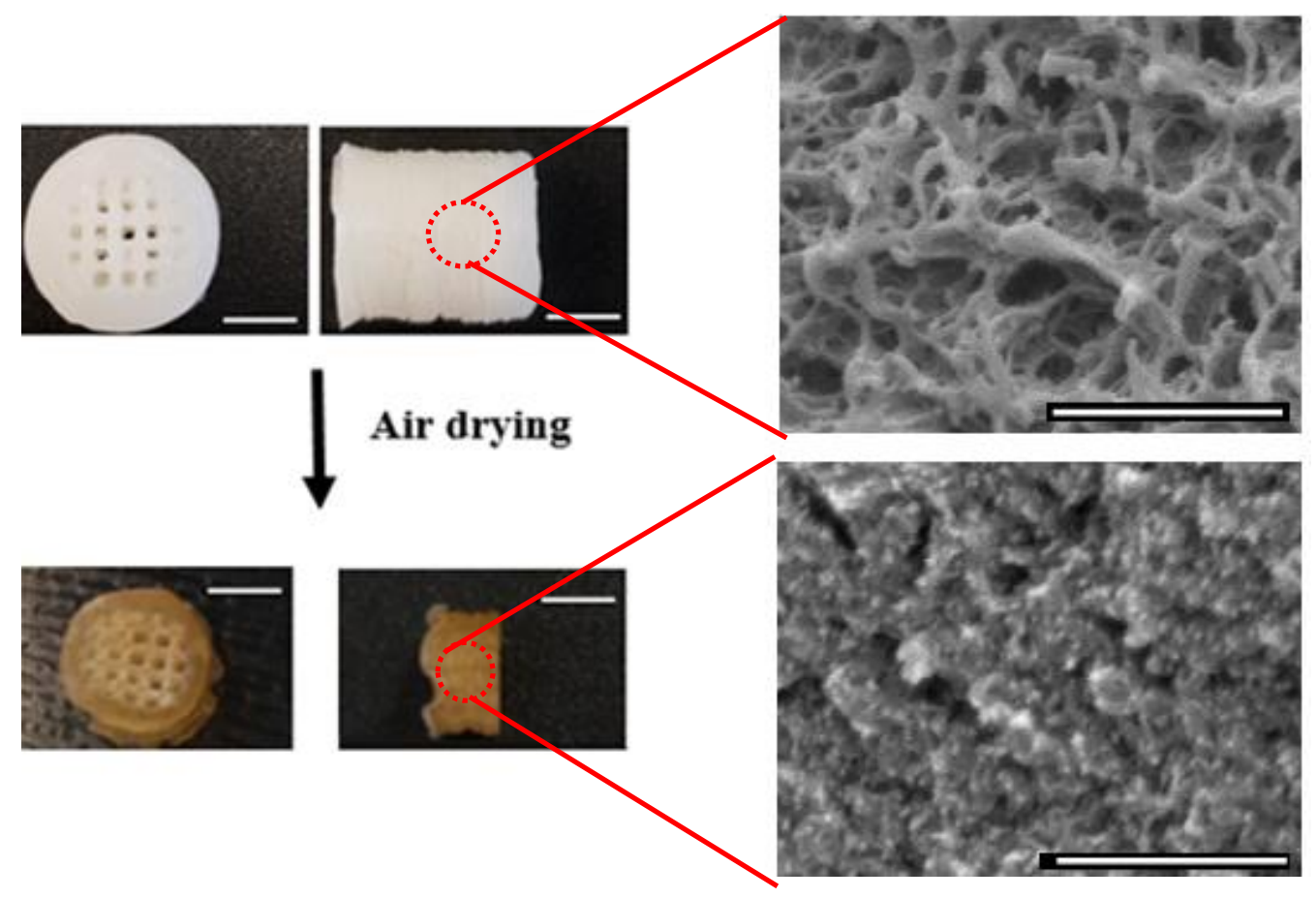

Figure 2.6: (a) Optical images of chitosan hydrogel-based scaffolds in the wet state, lefthand side, and on the right is the corresponding cryo-SEM image and (b) optical images of chitosan hydrogel-based scaffolds after air-drying, left-hand side, and on the right is the corresponding cryo-SEM image. The curling observed on the bottom of the dried scaffold is due to the first printed layer being slightly larger than the rest of the overlying layers of the scaffold. Thus, upon air-drying the first layer curls up due to the lack of both underlying layers and support from overlying layers of similar dimension (this issue is observed in all chitosan hydrogel-based 3D printed structures). Scale bar for the optical images is $0.5 \mathrm{~cm}$ and for the cryo-SEM images is 1 mm unless otherwise mentioned.

Figure 2.6 also shows the high-resolution electron microscope images of chitosan hydrogelbased scaffolds in both wet and dried states. Cryo-SEM images reveal that the physically crosslinked chitosan hydrogel-based scaffolds in their hydrated state are highly porous with 
interconnected nano to microscale pores. This along with the printed macropores results in these scaffolds showing hierarchal porosity from the nano to macroscale in the wet state. However, SEM images of dried scaffolds (Figure 2.6 (b)) reveal that air-drying results in the closure of most of the nano and micropores, although some are visible. However, majority of the final porosity of the dried scaffolds can be attributed only to the printed macropores (as designed by the CAD model).

Although some shrinkage was also observed in the case of the macroscale pores, air-drying did not lead to complete closure of these pores. After air-drying, the final macropores were $0.62 \pm 0.01 \mathrm{~mm}$ or $0.99 \pm 0.13 \mathrm{~mm}$ in size in comparison to the as printed $1.5 \mathrm{~mm} \times 1.5 \mathrm{~mm}$ or $2.0 \mathrm{~mm} \times 2.0 \mathrm{~mm}$ macropores in wet scaffolds. Literature suggests that the closure of micro and nanoscale pores is due to the surface tension effects of the evaporating water [42].

(a)

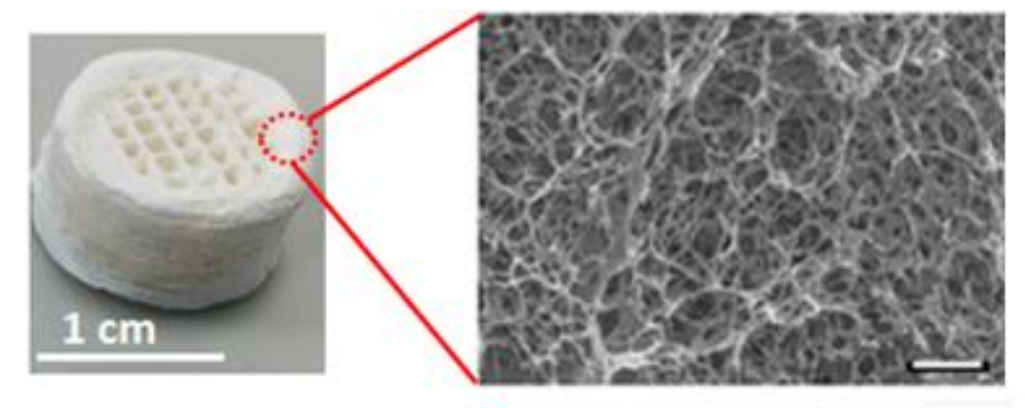

(b)

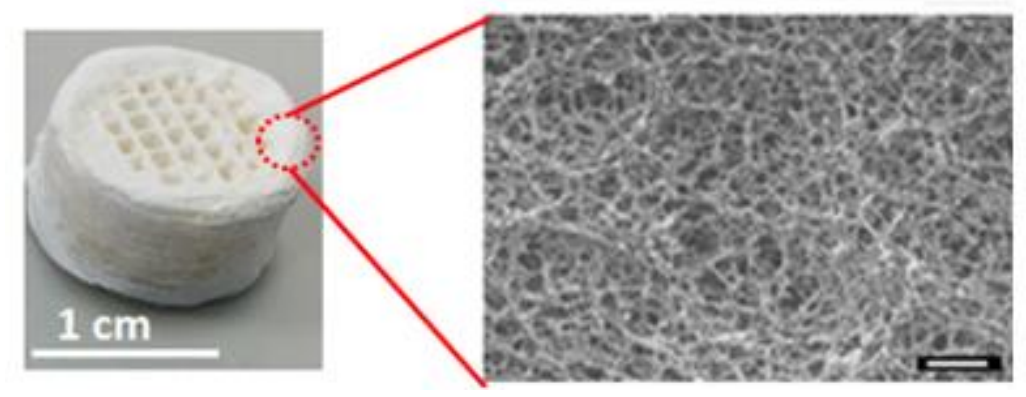

Figure 2.7: On the left are optical images of $3 D$ chitosan hydrogel-based scaffolds in the dried state, dried using one of the two drying techniques and the corresponding SEM images are shown on the right. (a) Critical point-drying and (b) freeze-drying. Scale bar in the image is 1 um unless otherwise mentioned.

Due to the significant shrinkage occurring during air-drying, other drying methods were explored; in particular critical point drying and freeze-drying. Figure 2.7 shows the optical and electron microscopy images of chitosan hydrogel-based scaffolds dried via these two methods. It was observed that unlike air-drying, freeze-drying and critical point-drying resulted in minimal shrinkage of the scaffolds dimensionally. Both the techniques resulted in dried scaffolds that are opaque white and were visually indistinguishable. SEM images 
revealed that these drying techniques maintained the intrinsic micro and nanopores of the hydrogel to a considerable extent. However, with freeze-dried scaffolds, a thick non-porous surface skin could be observed in the electron microscope. The formation of a surface skin is typical with freeze dried hydrogels due to pore collapse at the scaffold-air interface due to the interfacial tension caused by solvent evaporation [24].

As these scaffolds are envisioned to potentially be used as bone graft materials, their mechanical stability and strength are equally as important as the porosity, if not more. Although critical point and freeze-dried scaffolds showed high porosity covering a range of length scales, these scaffolds exhibited essentially no mechanical strength. Even applying a minimal compressive force exerted by squeezing the scaffolds between tweezer tips led to the scaffolds being irreversibly compressed by $>50 \%$ of their original volume. Air-dried scaffolds on the other hand exhibit high strength under similar conditions. Hence, freezedrying and critical point-drying were discounted as viable drying methods.

\subsubsection{Swelling behaviour of dried hydrogel-based scaffolds}

The swelling behaviour of hydrogels is driven by the restricted solubility of polymer chains and is dependent on: structure of the hydrogel (porous or non-porous); drying method used; polymer network density; crosslink density; solvent-polymer interactions; solvent $\mathrm{pH}$; and solvent nature [43-45]. Herein, swelling of dried scaffolds (i.e. increase in volume and mass due to solvent uptake) is expressed in terms of the solvent uptake ratio which is dependent on the solvent absorbance via diffusion into the scaffold when soaked in a medium for a prolonged period of time. Here this is defined as the ratio of the weight of the rehydrated scaffold to that of the dry scaffold. Table 2.1 gives the solvent uptake ratio and the percentage change in volume of chitosan hydrogel-based scaffolds subjected to various drying techniques after soaking in $1 \mathrm{X}$ PBS (pH 7.4) for one week.

The results show that freeze dried and critical point dried scaffolds (about 5) are able to absorb significant quantities of solvent in comparison to air-dried scaffolds with no physical change in their dimensions. This is due to the extensive porosity of these samples resulting from the nano, micro and macropores present in freeze dried and critical point dried scaffolds (Figure 2.7) in comparison to that of air-dried scaffolds that have predominantly macroscale pores. These microscale pores facilitate solvent diffusion throughout the scaffold via capillary action. 


\begin{tabular}{|c|c|c|c|}
\hline $\begin{array}{c}\text { Reswelling behaviour } \\
\text { in 1X PBS }\end{array}$ & Air-drying & Freeze-drying & $\begin{array}{c}\text { Critical point- } \\
\text { drying }\end{array}$ \\
\hline Solvent uptake ratio & $2.1 \pm 0.2$ & $5.2 \pm 1.0$ & $5.5 \pm 0.4$ \\
\hline$\%$ Volume change & $75.4 \pm 12.0$ & No change & No change \\
\hline
\end{tabular}

Table 2.1: Swelling behaviour of chitosan hydrogel-based scaffolds dried via different methods after soaking in $1 X$ PBS for one week. The solution was not changed during the experiment.
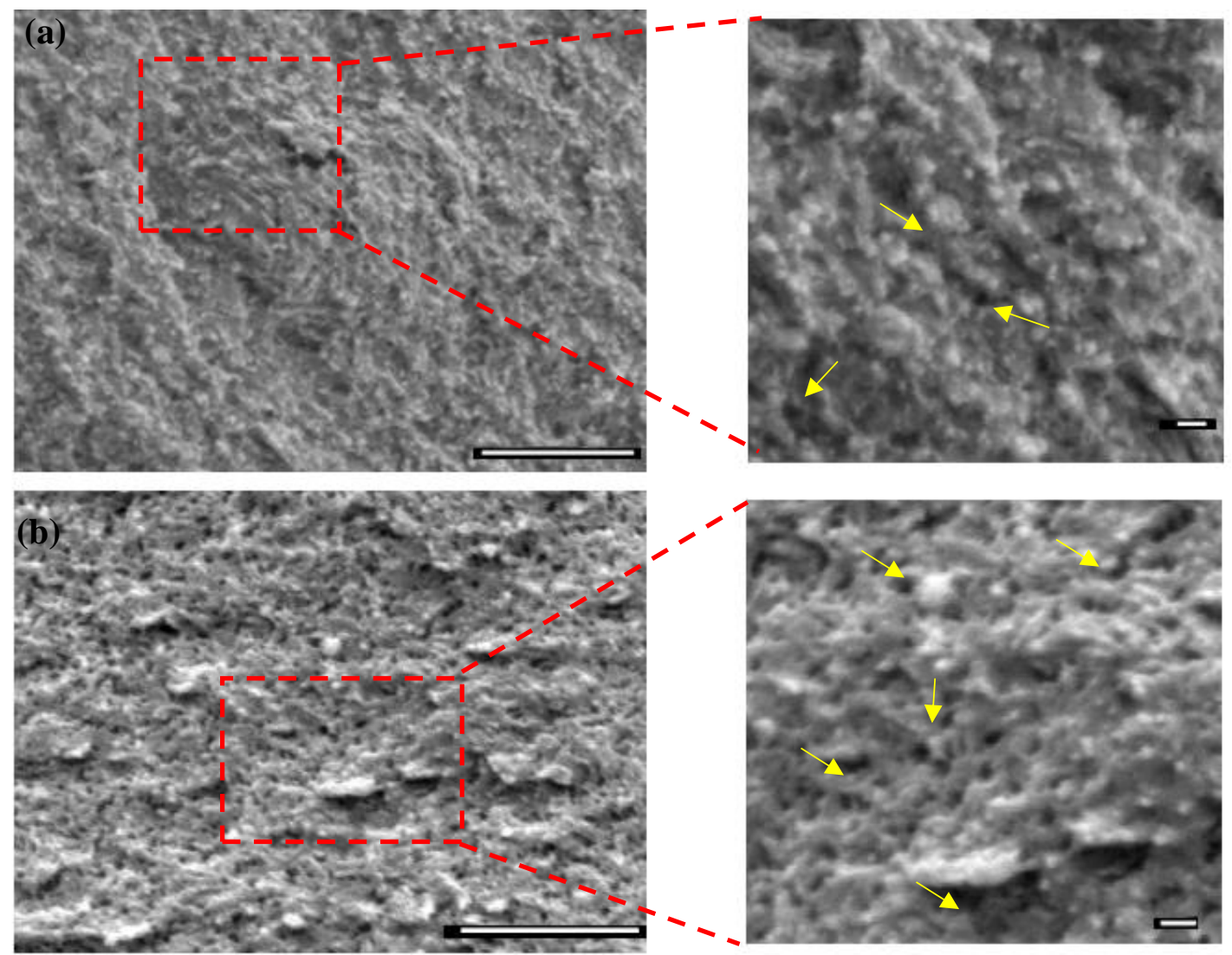

Figure 2.8: Cryo-SEM images of air-dried chitosan hydrogel-based scaffolds (a) before and (b) after soaking in IX PBS for 1 week. The yellow arrows point towards the nano/microscale pores within the region of observation. The scale bar for images on the left hand-side is 1 um and for those on the right-hand side is $100 \mathrm{~nm}$.

It was observed that the volume of freeze dried and critical point dried scaffold did not change with solvent diffusion, this could be due to the fact that the chitosan polymer chains were rendered crystalline as a result of critical point-drying or freeze-drying. This inhibits any chain dissolution dependent swelling and hence causes no apparent change in the 
volume of the scaffold [16]. In the case of air-dried scaffolds, the increase in the volume of the scaffolds after soaking suggests that air-drying does not render the polymer chains completely crystalline leading to a dissolution dependent swelling of the polymer chains. Cryo-SEM images of rehydrated air-dried chitosan hydrogel-based scaffolds support this hypothesis. A large number of micro to nanoscale pores are revealed in the rehydrated scaffolds in comparison to the air-dried scaffolds (Figure 2.8), indicating that some of the porosity that was "lost" during air-drying is regained when the scaffolds are rehydrated. However, in this case, the lack of an extensive interconnected microporous network as observed in freeze-dried or critical point-dried samples (Figure 2.7), resulted in a comparatively lower solvent uptake ratio. It should be noted that swelling did not result in regaining the porosity observed in the initial as fabricated chitosan hydrogel-based scaffold in it wet state (Figure 2.6 (a)). Nonetheless, in vitro culture studies suggest that swelling and the resultant increase in the porosity of dry materials will enhance the cell attachment and growth within such 3D structures [46].

\subsubsection{Porosity analysis}

The pores within a 3D scaffold can be categorised as open or closed. Open pores are those which are connected to the outside and closed pores are those which are completely surrounded by objects and is therefore not accessible from the outside. Various methods can be used to determine the porosity of a 3D structure such as the gravimetric method, mercury porosimetry, scanning electron microscopy and the liquid displacement method [31]. However, methods such as mercury porosimetry do not account for closed pores present in a 3D system. Also, since the 3D printed chitosan hydrogel-based scaffolds shrink irregularly when air-dried, it is not possible to accurately measure the volume of the final dried scaffolds, making it difficult to estimate the porosity via these traditional methods.

Micro-CT analysis can give a good estimation of both open as well as closed pores within a 3D sample using the degree of X-ray attenuation at different regions within the scaffold when irradiated with X-rays of a particular energy. This technique is non-destructive and hence the same sample can be used for further tests. The total porosity (open + closed pores) of the dried hydrogel-based scaffolds was calculated using the same method as that used to calculate the porosity and obtain a 3D image of the closed and open pores of a rock sample as described in references $[47,48]$. Figure 2.9 shows a flowchart of the steps performed to 
calculate the porosity of an air-dried chitosan hydrogel-based scaffold with $1.5 \mathrm{~mm} \times 1.5$ mm macropore using Skyscan CTAn software.

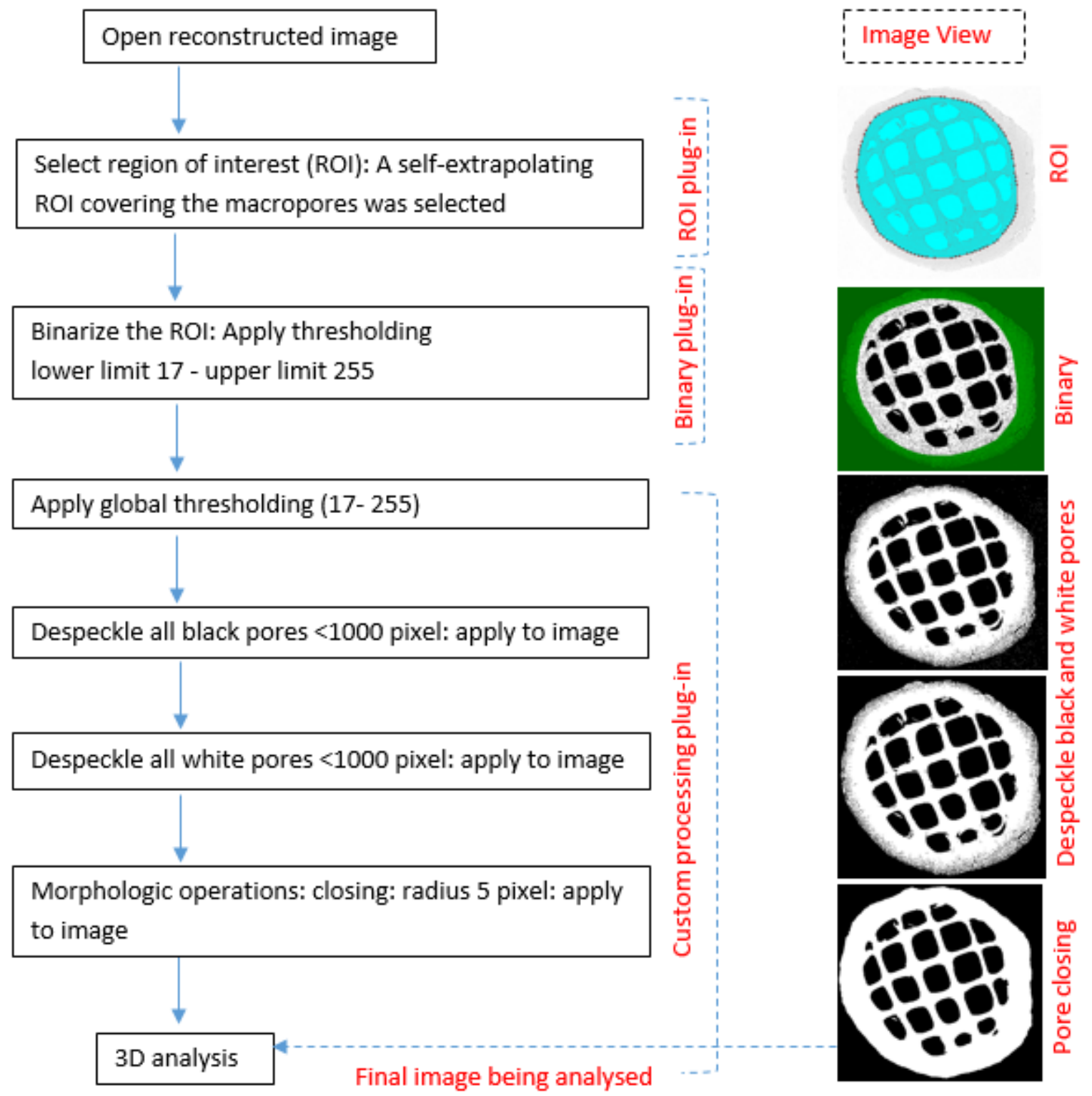

Figure 2.9: A flowchart of the steps performed to calculate the total porosity of the $3 D$ hydrogel scaffolds using the Skyscan CT analysis software. Only one of the reconstructed slices (590 slices in total) of a whole air-dried 3D printed chitosan hydrogel-based scaffold with $1.5 \mathrm{~mm} \times 1.5 \mathrm{~mm}$ macropores (as in CAD model), scanned using a Bruker Skyscan 1272, is shown as a representative image on the left-hand side.

The SEM analysis indicated that the nano and microscale pores present within the 3D chitosan hydrogel-based scaffold in their wet state, collapse due to surface tension effects during solvent evaporation when air-drying is used. The collapse of the micropores was confirmed in the $2 \mu \mathrm{m}$ resolution micro-CT scans with the only pores contributing to the 
total porosity of the final dried chitosan hydrogel-based scaffold being the open macropores and any micron-size closed pores that might be present within the 3D structure. 3D reconstruction of the micro-CT images confirmed this conclusion.

In micro-CT analysis, all black pixels are considered as pores and white pixels as objects. Due to noise certain small pixels may appear as a white (object) or black (pores) and so despeckling is performed in order to remove any such unwanted pixels as it will otherwise affect the final porosity calculation. The final morphological "closing" operation essentially removes black pixels which are smaller than a disc of predefined area (radius 5 pixels in this case), this essentially means opening up the white pixels around the tiny noise-like collection of black pixels, cleaning up any unwanted black pixels that remain after the despeckling process [49]. Figure 2.10 shows a 3D visualization of open and closed pores in the region of interest (ROI) selected within an air-dried 3D chitosan hydrogel-based scaffold with $2.0 \mathrm{~mm} \times 2.0 \mathrm{~mm}$ macropores (macropore dimension when in the wet state). Open pores predominantly contribute to the porosity of the final structure, however, there is a very small number of contributing closed pores that are also evident.
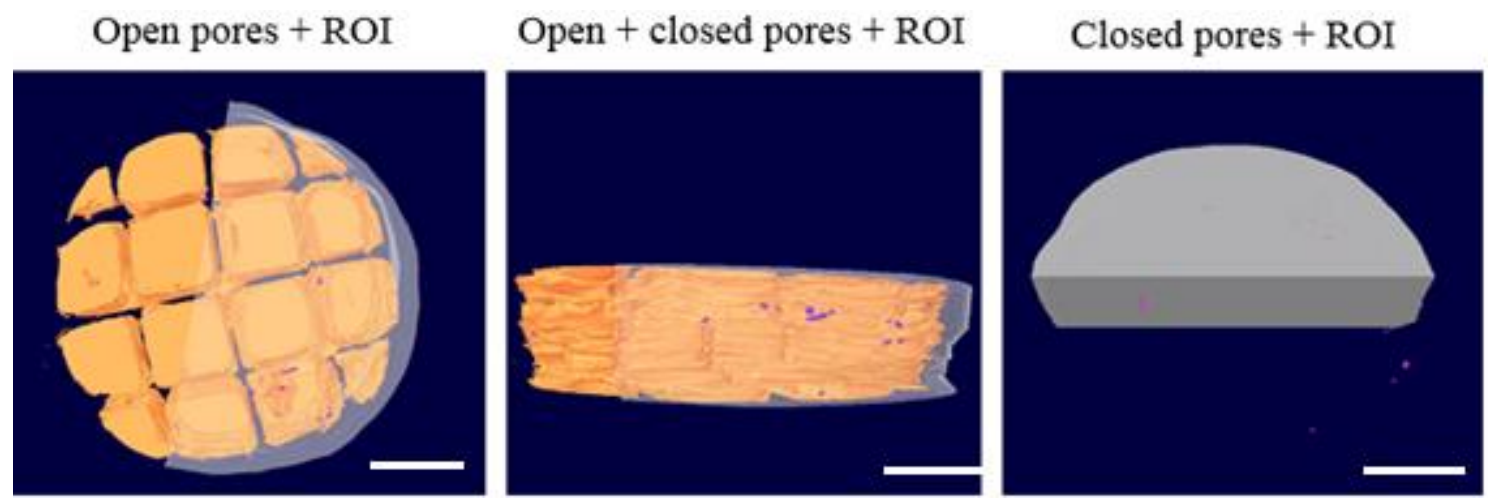

Figure 2.10: $3 D$ visualization (top view and side view) of the open pores (orange) and closed pores (magenta) inside the ROI (translucent-grey) selected within the reconstructed micro-CT image of a 3D chitosan hydrogel-based scaffold with $2 \mathrm{~mm} \times 2 \mathrm{~mm}$ macropores (macropore dimensions are based on the CAD model used and may differ in the final printed and dried structure due to shrinkage as described previously. Scale bar is $1 \mathrm{~cm}$.

Table 2.2 gives a comparison between the porosity of dried chitosan hydrogel-based scaffolds with $1.5 \mathrm{~mm} \times 1.5 \mathrm{~mm}$ and $2 \mathrm{~mm} \times 2 \mathrm{~mm}$ macropores in wet state calculated based on the CAD model and micro-CT image analysis using Eq 2.6. It should be noted that scaffolds were air-dried prior to micro-CT analysis and the final pore dimension is $0.62 \pm$ $0.01 \mathrm{~mm}$ or $0.99 \pm 0.13 \mathrm{~mm}$ respectively. The variation in the porosity estimated based on 
the CAD model and that from the micro-CT images could be attributed to the closure of nano and microscale pores and the subsequent effect on the degree of shrinkage of the macropores and the total object volume; the semi-automated nature of the printing process introducing structural defects such as print gaps; or closed pores due to filling of some very small macropores due to hydrogel flow among other thing. All of these things may have contributed to a decrease in the total object volume and thus leading to an increase the pore space within the scaffold.

\begin{tabular}{|c|c|c|}
\hline $\begin{array}{c}\text { Chitosan hydrogel-based scaffold } \\
\text { (macropore dimensions) }\end{array}$ & CAD model (\%) & Micro-CT (\%) \\
\hline $1.5 \mathrm{~mm} \times 1.5 \mathrm{~mm}$ & 34.4 & $51.6 \pm 1.3$ \\
\hline $2.0 \mathrm{~mm} \times 2.0 \mathrm{~mm}$ & 43.0 & $58.8 \pm 1.2$ \\
\hline
\end{tabular}

Table 2.2: The porosity of $3 D$ printed chitosan hydrogel-based (dried) with two different macro-pore dimensions, estimated based on their respective CAD model (15 mm diameter $\times 16 \mathrm{~mm}$ height hollow cylinder + cubic lattice structure) and by using $2 \mathrm{D}$ micro-CT analysis.

Both methods provide relatively similar estimates. Nonetheless, micro-CT gives the best approximation of the total porosity of 3D scaffold. Literature suggests that the values obtained in the case of the chitosan hydrogel-based scaffolds with either macropore dimension are within the range that is suitable for osteoblast cell proliferation, as was the goal of this research $[50,51]$. Therefore, considering the implications of both mechanical strength as described in the previous section and the porosity of a final dried 3D scaffold with regard to its in vivo functionality, air-drying was chosen as a suitable drying technique for 3D printed chitosan hydrogel-based scaffolds. Additionally, based on the swelling behaviour of air-dried chitosan hydrogel-based scaffolds at $\mathrm{pH} 7.4$, which is similar to that of biofluids, it can also be deducted that the porosity of the scaffolds will further increase under in vivo conditions, which is found to be advantageous for 3D cell attachment and bone ingrowth. 


\subsection{Conclusion}

The prospect of using chitosan hydrogel as the printing ink in a custom-designed nozzle extrusion-based 3D printer to fabricate symmetrically porous 3D scaffolds was investigated. The rheological properties of chitosan hydrogels of various concentrations were studied and a concentration suitable to be used as the printing ink in our custom-designed 3D printer was identified. This study forms the basis for the smooth transition from the use of $2 \mathrm{D}$ templates fabricated previously in the McGrath group to 3D scaffolds using chitosan hydrogels in the fabrication of composites biomimetic materials. $5 \% \mathrm{w} / \mathrm{v}$ chitosan in $2 \% \mathrm{v} / \mathrm{v}$ acetic acid solution produced a printing ink that was suitable. Its flow properties under both increasing shear rate and oscillation frequency were best suited for the extrusion parameters of the 3D printer when using an extrusion nozzle with $0.42 \mathrm{~mm}$ internal diameter. This hydrogel ensured that extrusion of the gel, in the form of a continuous strand, occurred and that hence accurate printing of the input CAD model was achieved.

The CAD model chosen for this work was a combination of a hollow cylinder enclosing a symmetric cubic lattice-based 3D structure. This model was selected based on the mechanical testing of a range of model 3D structures that were printed using ABS-based filaments. The selected structure was envisioned to fulfil both the porosity and the mechanical strength attributes of the final 3D structure, thus being able to be used in the development of bone graft materials.

The architecture and structural stability of the final printed 3D chitosan hydrogel-based scaffolds under different drying conditions: air-drying; freeze-drying; and critical pointdrying were analysed. The 3D scaffolds were structurally stable in both wet and dry conditions. In the wet condition 3D chitosan hydrogel-based scaffolds were inherently highly porous with interconnected micro and nanoscale pores along with the printed macropores. Although air-drying resulted in considerable dimensional shrinkage and also the closure of a majority of the micro and nanopores, air-drying was chosen as a suitable technique to dry the chitosan hydrogel-based scaffolds based on the observation that airdried scaffolds retained the printed macropores which provided a minimum porosity of $50 \%$ in the case of the scaffolds printed with $1.5 \mathrm{~mm} \times 1.5 \mathrm{~mm}$ macropores and $58 \%$ in the case of those printed with $2.0 \mathrm{~mm} \times 2.0 \mathrm{~mm}$ macropores. This porosity is within the range suitable for in vivo applications. Also, these scaffolds exhibited appreciable mechanical strength, which was deemed to be sufficient to warrant further investigation (Chapter 5). 
Additionally, air-dried scaffolds exhibit good swelling behaviour in 1X PBS (pH 7.4, (which is comparable to that of body fluids) resulting in regaining some of the "lost" porosity as a result of the pore collapse during air-drying in comparison to when samples were freeze-dried or critical point-dried. This suggests that the porosity of scaffolds will increase in vivo due to swelling, hence aiding in increased solvent exchange and cell attachment as well as aiding in biodegradation of the scaffold in vivo.

The results suggest that $3 \mathrm{D}$ printing is an effective fabrication technique for developing structured chitosan hydrogel-based scaffolds. Air-drying is an effective method for drying the scaffolds resulting in mechanically strong and porous scaffolds and thereby providing a pathway towards bone graft applications.

The effects of introducing inorganic components within 3D printed porous chitosan hydrogel-based scaffolds via different mineralization methods on the mechanical strength and biological properties of the final composite material were investigated and are presented in Chapters 3-6. 


\subsection{Bibliography}

1. Gattazzo, F., A. Urciuolo, and P. Bonaldo, Extracellular matrix: a dynamic microenvironment for stem cell niche. Biochimica et Biophysica Acta (BBA)-General Subjects, 2014. 1840(8): p. 2506-2519.

2. Schubert, C., M.C. van Langeveld, and L.A. Donoso, Innovations in 3D printing: a 3D overview from optics to organs. British Journal of Ophthalmology, 2013: p. bjophthalmol-2013-304446.

3. Ozbolat, I.T. and Y. Yu, Bioprinting toward organ fabrication: challenges and future trends. Biomedical Engineering, IEEE Transactions on, 2013. 60(3): p. 691-699.

4. Ventola, C.L., Medical applications for 3D printing: current and projected uses. PT, 2014. 39(10): p. 704-711.

5. Amini, A.R., C.T. Laurencin, and S.P. Nukavarapu, Bone tissue engineering: recent advances and challenges. Critical Reviews ${ }^{\mathrm{TM}}$ in Biomedical Engineering, 2012. 40(5).

6. Costa-Pinto, A.R., R.L. Reis, and N.M. Neves, Scaffolds based bone tissue engineering: the role of chitosan. Tissue Engineering Part B: Reviews, 2011. 17(5): p. 331-347.

7. Geng, L., Y. Wong, D. Hutmacher, W. Feng, H. Loh, and J. Fuh, Rapid Prototyping of $3 D$ Scaffolds for Tissue Engineering Using a Four-Axis Multiple-Dispenser Robotic System. Dept. of Mechanical Engineering, National University of Singapore, Singapore, 2003.

8. Sun, W., B. Starly, J. Nam, and A. Darling, Bio-CAD modeling and its applications in computer-aided tissue engineering. Computer-Aided Design, 2005. 37(11): p. 10971114.

9. Matsuda, T. and M. Mizutani, Liquid acrylate-endcapped biodegradable poly ( $\epsilon$ caprolactone-co-trimethylene carbonate). II. Computer-aided stereolithographic microarchitectural surface photoconstructs. Journal of Biomedical Materials Research, 2002. 62(3): p. 395-403.

10. Hutmacher, D.W., M. Sittinger, and M.V. Risbud, Scaffold-based tissue engineering: rationale for computer-aided design and solid free-form fabrication systems. TRENDS in Biotechnology, 2004. 22(7): p. 354-362.

11. Vail, N., L. Swain, W. Fox, T. Aufdlemorte, G. Lee, and J. Barlow, Materials for biomedical applications. Materials \& Design, 1999. 20(2): p. 123-132. 
12. Sachs, E.M., J.S. Haggerty, M.J. Cima, and P.A. Williams, Three-dimensional printing techniques. 1993, Google Patents.

13. Hutmacher, D.W., T. Schantz, I. Zein, K.W. Ng, S.H. Teoh, and K.C. Tan, Mechanical properties and cell cultural response of polycaprolactone scaffolds designed and fabricated via fused deposition modeling. Journal of Biomedical Materials Research, 2001. 55(2): p. 203-216.

14. Zein, I., D.W. Hutmacher, K.C. Tan, and S.H. Teoh, Fused deposition modeling of novel scaffold architectures for tissue engineering applications. Biomaterials, 2002. 23(4): p. 1169-1185.

15. Liu, W., Y. Li, J. Liu, X. Niu, Y. Wang, and D. Li, Application and performance of $3 D$ printing in nanobiomaterials. Journal of Nanomaterials, 2013. 2013: p. 13.

16. A Nawawi, N., A. SF Alqap, and I. Sopyan, Recent progress on hydroxyapatite-based dense biomaterials for load bearing bone substitutes. Recent Patents on Materials Science, 2011. 4(1): p. 63-80.

17. Ang, T., F. Sultana, D. Hutmacher, Y.S. Wong, J. Fuh, X. Mo, H.T. Loh, E. Burdet, and S.-H. Teoh, Fabrication of 3D chitosan-hydroxyapatite scaffolds using a robotic dispensing system. Materials Science and Engineering: C, 2002. 20(1): p. 35-42.

18. Lim, T.C., K.S. Chian, and K.F. Leong, Cryogenic prototyping of chitosan scaffolds with controlled micro and macro architecture and their effect on in vivo neovascularization and cellular infiltration. Journal of Biomedical Materials Research Part A, 2010. 94(4): p. 1303-1311.

19. Lee, H. and G. Kim, Cryogenically fabricated three-dimensional chitosan scaffolds with pore size-controlled structures for biomedical applications. Carbohydrate Polymers, 2011. 85(4): p. 817-823.

20. Haberstroh, K., K. Ritter, J. Kuschnierz, K.H. Bormann, C. Kaps, C. Carvalho, R. Mülhaupt, M. Sittinger, and N.C. Gellrich, Bone repair by cell-seeded 3D-bioplotted composite scaffolds made of collagen treated tricalciumphosphate or tricalciumphosphate-chitosan-collagen hydrogel or PLGA in ovine critical-sized calvarial defects. Journal of Biomedical Materials Research Part B: Applied Biomaterials, 2010. 93(2): p. 520-530.

21. Munro, N.H. and K.M. McGrath, Biomimetic approach to forming chitin/aragonite composites. Chemical Communications, 2012. 48(39): p. 4716-4718. 
22. Atlan, G., O. Delattre, S. Berland, A. LeFaou, G. Nabias, D. Cot, and E. Lopez, Interface between bone and nacre implants in sheep. Biomaterials, 1999. 20(11): p. 1017-1022.

23. Whang, K., K. Healy, D. Elenz, E. Nam, D. Tsai, C. Thomas, G. Nuber, F. Glorieux, R. Travers, and S. Sprague, Engineering bone regeneration with bioabsorbable scaffolds with novel microarchitecture. Tissue Engineering, 1999. 5(1): p. 35-51.

24. Venkatesan, J. and S.-K. Kim, Chitosan composites for bone tissue engineering - an overview. Marine Drugs, 2010. 8(8): p. 2252-2266.

25. Gooch, J.W., Hagen-Poiseuille equation, in Encyclopedic Dictionary of Polymers. 2011, Springer. p. 355-355.

26. Suntornnond, R., E.Y.S. Tan, J. An, and C.K. Chua, A mathematical model on the resolution of extrusion bioprinting for the development of new bioinks. Materials, 2016. 9(9): p. 756.

27. Gough, J.E., C.A. Scotchford, and S. Downes, Cytotoxicity of glutaraldehyde crosslinked collagen/poly (vinyl alcohol) films is by the mechanism of apoptosis. Journal of Biomedical Materials Research Part A, 2002. 61(1): p. 121-130.

28. Lai, J.-Y., Biocompatibility of genipin and glutaraldehyde cross-linked chitosan materials in the anterior chamber of the eye. International Journal of Molecular Sciences, 2012. 13(9): p. 10970-10985.

29. Dahle, J., E. Kvam, and T. Stokke, Bystander effects in UV-induced genomic instability: antioxidants inhibit delayed mutagenesis induced by ultraviolet $A$ and $B$ radiation. Journal of Carcinogenesis, 2005. 4(1): p. 11.

30. Deepthi, S., J. Venkatesan, S.-K. Kim, J.D. Bumgardner, and R. Jayakumar, An overview of chitin or chitosan/nano ceramic composite scaffolds for bone tissue engineering. International Journal of Biological Macromolecules, 2016. 93: p. 13381353.

31. Loh, Q.L. and C. Choong, Three-dimensional scaffolds for tissue engineering applications: role of porosity and pore size. Tissue Engineering Part B: Reviews, 2013. 19(6): p. 485-502.

32. Ho, S.T. and D.W. Hutmacher, A comparison of micro CT with other techniques used in the characterization of scaffolds. Biomaterials, 2006. 27(8): p. 1362-1376.

33. Barrett, J.F. and N. Keat, Artifacts in CT: recognition and avoidance. Radiographics, 2004. 24(6): p. 1679-1691. 
34. Ouyang, L., C.B. Highley, C.B. Rodell, W. Sun, and J.A. Burdick, 3D printing of shearthinning hyaluronic acid hydrogels with secondary cross-linking. ACS Biomaterials Science \& Engineering, 2016. 2(10): p. 1743-1751.

35. Raposo, S., A. Salgado, G. Eccleston, M. Urbano, and H.M. Ribeiro, Cold processed oil-in-water emulsions for dermatological purpose: formulation design and structure analysis. Pharmaceutical Development and Technology, 2014. 19(4): p. 417-429.

36. EL-HAFIAN, E.A., E.S. Elgannoudi, A. Mainal, and A.H.B. Yahaya, Characterization of chitosan in acetic acid: Rheological and thermal studies. Turkish Journal of Chemistry, 2010. 34(1): p. 47-56.

37. An, Y.H. and R.A. Draughn, Mechanical testing of bone and the bone-implant interface. 1999: CRC press.

38. Sadeghi, H., D.M. Espino, and D.E. Shepherd, Variation in viscoelastic properties of bovine articular cartilage below, up to and above healthy gait-relevant loading frequencies. Proceedings of the Institution of Mechanical Engineers, Part H: Journal of Engineering in Medicine, 2015. 229(2): p. 115-123.

39. Levengood, S.K.L. and M. Zhang, Chitosan-based scaffolds for bone tissue engineering. Journal of Materials Chemistry B, 2014. 2(21): p. 3161-3184.

40. Griffin, D., Inelastic and creep buckling of circular cylinder due to axial compression, bending, and twisting. 1973, Westinghouse Electric Corp., Pittsburgh, Pa.(USA). Advanced Reactors Div.

41. Wieding, J., A. Jonitz, and R. Bader, The effect of structural design on mechanical properties and cellular response of additive manufactured titanium scaffolds. Materials, 2012. 5(8): p. 1336-1347.

42. Ahmed, E.M., Hydrogel: Preparation, characterization, and applications: A review. Journal of Advanced Research, 2015. 6(2): p. 105-121.

43. Meldrum, F., Calcium carbonate in biomineralisation and biomimetic chemistry. International Materials Reviews, 2003. 48(3): p. 187-224.

44. He, F., W. Ren, X. Tian, W. Liu, S. Wu, and X. Chen, Comparative study on in vivo response of porous calcium carbonate composite ceramic and biphasic calcium phosphate ceramic. Materials Science and Engineering: C, 2016. 64: p. 117-123.

45. Florencio-Silva, R., G.R.d.S. Sasso, E. Sasso-Cerri, M.J. Simões, and P.S. Cerri, Biology of bone tissue: structure, function, and factors that influence bone cells. BioMed Research International, 2015. 2015. 
46. Li, Z., H.R. Ramay, K.D. Hauch, D. Xiao, and M. Zhang, Chitosan-alginate hybrid scaffolds for bone tissue engineering. Biomaterials, 2005. 26(18): p. 3919-3928.

47. Bruker. Introduction to porosity analysis. Available from: http://umanitoba.ca/faculties/health sciences/medicine/units/cacs/sam/media/MN059 _PorosityAnalysis.pdf.

48. Bruker. $3 D$ visualization of open and closed porosity. Available from: http://www.umanitoba.ca/faculties/health_sciences/medicine/units/cacs/sam/media/M N010_3D_visualization_of_open_and_closed_porosity.pdf.

49. Glasbey, C.A. and G.W. Horgan, Image analysis for the biological sciences. Vol. 1. 1995: Wiley Chichester.

50. Hing, K.A., Bioceramic bone graft substitutes: influence of porosity and chemistry. International Journal of Applied Ceramic Technology, 2005. 2(3): p. 184-199.

51. Roseti, L., V. Parisi, M. Petretta, C. Cavallo, G. Desando, I. Bartolotti, and B. Grigolo, Scaffolds for Bone Tissue Engineering: State of the art and new perspectives. Materials Science and Engineering: C, 2017. 78: p. 1246-1262. 


\section{Minerlization of Chitosan Hydrogel-based Scaffolds}

\subsection{Introduction}

Biocompatible polymers such as chitosan, collagen, gelatine or polyurethene and ceramics such as calcium phosphates are often combined for bone tissue engineering applications in order to mitigate the disadvantages such as the brittle nature and poor processability of the ceramics and low compressive strength and osteoconductivity of the biopolymers (in some cases) [1-3]. Calcium phosphates are the most commonly studied mineral phase in biocomposites owing to their similarity to the bone mineral, but some calcium carbonates derived from corals (along with other important fluorides) have also been reported to show good osteoconductivity and bioresorption $[4,5]$.

Native chitosan hydrogels are inherently biocompatible but lack the mechanical strength to be considered for load-bearing bone graft applications. Different methods have been reported in the literature whereby minerals are incorporated within chitosan-based hydrogel matrices through for example physical blending, co-precipitation, soaking in simulated body fluids or double diffusion methods $[6,7]$. This work investigates mineralization of 3D chitosan hydrogel-based scaffolds via in situ mineralization using the McGrath method, in order to grow calcium carbonate crystallites as filler particles within the organic matrix of the 3D scaffold. The McGrath mineralization method was developed in an effort to mimic nacre in a laboratory scale. Owing to the optical properties and biological significance of nacre, which is a chitin-calcium carbonate composite, a number of approaches have been applied in order to mimic nacre on a laboratory scale. For example, the principles of, supramolecular assembly, alternate soaking, Langmuir-Blodgett films, self-assembled multi-layered films, sequential deposition and freeze casting, have all been used [8-14].

Research previously undertaken in the McGrath group has shown that $\mathrm{CaCO}_{3}$ crystallites, mimicking the morphology of nacre in its early stages of development, can be developed within a 2D chitosan hydrogel-based template via the McGrath method (explained in Chapter 1) [15]. This is shown in Figure 3.1 where electron microscopy images are shown 
for the calcium carbonate growth achieved with 2D chitosan hydrogel-based films and that occurring in nacre in its early stages of development.
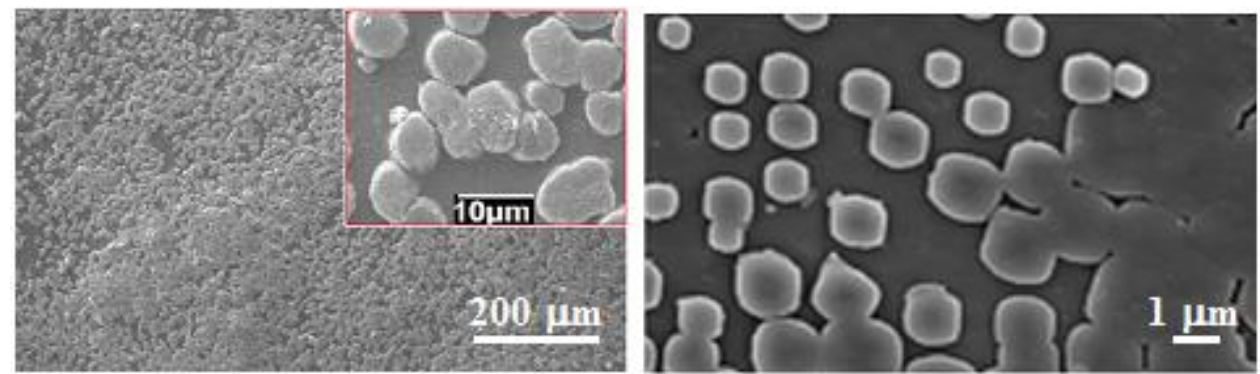

Figure 3.1: SEM image of calcium carbonate crystallites developed on 2D chitosan hydrogel-based template via the McGrath mineralization method in the presence of polyacrylic acid on the left and that of nacre in its early stages of development (reprinted with permission from [16]).

Crystal growth modifiers can be used to tailor the $\mathrm{CaCO}_{3}$ precipitation as well as the morphology of the resulting crystallites. These chemical additives are used to mimic the role of certain soluble proteins involved in the biomineralization processes in molluscs for example, wherein these proteins interact with the growing calcium carbonate crystallites resulting in the formation of flat platelets of calcium carbonate existing as aragonite single crystals, a less stable polymorph of $\mathrm{CaCO}_{3}$ compared to calcite $[17,18]$. It was found that the introduction of polyacrylic acid (PAA) as the crystal growth modifier into growth solutions when using the McGrath mineralization method, resulted in the formation of nacre-like calcium carbonate crystallites in 2D chitosan hydrogel-based films.

In this Chapter, the effective translation of the mineralization achieved within 2D chitosan hydrogel-based films to 3D porous chitosan hydrogel-based scaffolds is explored. A 3D system can be seen as a stack of 2D templates built along the Z-axis. Hence, by applying a similar synthesis technique while introducing no stress-induced effects on the growing crystallites due to the overlying layers, it can be presumed that the growth of the crystallites within the chitosan hydrogel matrix will follow similar pathways in both the 2D and 3D systems. As such, it is proposed that the McGrath method will facilitate the formation of flat platelet-like calcium carbonate crystallites within each layer of the 3D print, which on a macroscale, can be visualised as thin layers (macroscale) of organic matrix interlacing micron-sized $\mathrm{CaCO}_{3}$ crystallites. Hence, replicating the "brick-mortar" architecture of nacre 
(thin single crystal platelets of $\mathrm{CaCO}_{3}$ stacked over each other interlaced by thin layers of the organic matrix) on a higher length scale.

In this Chapter,

(a) the strategies adopted to effectively translate the mineralization achieved in $2 \mathrm{D}$ chitosan hydrogel-based films to a 3D chitosan hydrogel-based scaffold, when using the McGrath method, are explained;

(b) an appropriate concentration of crystal growth modifier required to form the desired calcium carbonate morphology throughout the 3D scaffold is determined by analyzing the effect of increasing concentration of the same on the morphology of the resultant crystallites formed on the exposed regions and in between the layers of the scaffold; and

(c) the effect of varying various components of the McGrath mineralization method and the scaffold architecture on the extent of mineralization achieved in between the printed layers of the 3D scaffold is investigated using scanning electron microscopy (SEM), energy dispersive spectroscopy (EDS), X-ray diffraction (XRD) and thermogravimetric analysis (TGA). 


\subsection{Materials and methods}

The chitosan hydrogel-based scaffolds, fabricated via 3D printing as described in Chapter 2, were mineralized using the McGrath method. The physical and structural properties of the composites thus fabricated were studied using SEM, EDS, XRD and TGA.

\subsubsection{Materials}

Analytical grade chitosan (MW: 100,000 - 300,000 g/mol), calcium chloride dihydrate $\left(\mathrm{CaCl}_{2} \cdot 2 \mathrm{H}_{2} \mathrm{O}\right)$, and polyacrylic acid (PAA, MW: average $1800 \mathrm{~g} / \mathrm{mol}$ ) were purchased from Sigma Aldrich. Acetic acid (Univar, $\mathrm{AR}$ grade), $\mathrm{CaCO}_{3}$ (Univar, $\mathrm{AR}$ grade), and sodium hydrogen carbonate $\left(\mathrm{NaHCO}_{3}\right)$ (Pure science) were used as received. All solutions used in this work were prepared using water purified using a Sartorius arium 611UV purification system, (18.2 $\mathrm{M} \Omega \mathrm{cm}$ resistivity).

\subsubsection{Mineralization}

3D printed chitosan hydrogel-based scaffolds were mineralized using the McGrath method. The McGrath method is the combination of the alternate soaking method with the Kitano method as described in Chapter 1 [15]. The former involves alternatively soaking the $\mathrm{pH}$ neutralized scaffolds for $24 \mathrm{~h}$ in $25 \mathrm{~mL}$ aliquots of solutions of the mineral precursors for $\mathrm{CaCO}_{3}$ nucleation. The precursor ion solutions used were calcium chloride dihydrate and sodium hydrogen carbonate (bubbled with $\mathrm{CO}_{2}$ at $3 \mathrm{~L} \mathrm{~h}^{-1}$ for $15 \mathrm{~min}$ ). The now pre-loaded scaffold was then soaked in $25 \mathrm{~mL}$ aliquots of a saturated solution of $\mathrm{CaCO}_{3}\left(\mathrm{CO}_{2}\right.$ bubbled at $3 \mathrm{~L} \mathrm{~h}^{-1}$ for $15 \mathrm{~min}$ ), so-called "Kitano" solution, for a period of 7 days. The saturated solution of $\mathrm{CaCO}_{3}$ was prepared by dissolving $2.6 \mathrm{~g}$ of calcium carbonate in $1 \mathrm{~L}$ of purified water under $\mathrm{CO}_{2}$ bubbling at $3 \mathrm{~L} \mathrm{~h}^{-1}$ for $8 \mathrm{~h}$. The solution was then filtered, and the filtrate was further $\mathrm{CO}_{2}$ bubbled for another $30 \mathrm{~min}$ at $3 \mathrm{~L} \mathrm{~h}^{-1}$.

To study the effect of additives/crystal growth modifiers on the morphology of calcium carbonate crystallites within the final composite, a modified mineralization protocol was followed. PAA was used as an additive/crystal growth modifier and was incorporated into every step of the mineralization process. To study its effect on the crystal morphology, the concentration of PAA incorporated was varied from $0.5 \%-3.5 \%$ w/w w.r.t the dry weight of the chitosan scaffold. The $\mathrm{pH}$ of the individual mineralization solutions at various steps during the McGrath mineralization process are given in Table 3.1. 


\begin{tabular}{|c|c|c|}
\hline Mineralization solution & $\begin{array}{c}\text { pH after } \mathrm{CO}_{2} \text { bubbling } \\
\text { (before addition of PAA) }\end{array}$ & $\begin{array}{c}\text { pH after addition of } \\
\text { 2.5\% PAA }\end{array}$ \\
\hline $\mathrm{CaCl}_{2} .2 \mathrm{H}_{2} \mathrm{O}(0.5 \mathrm{M})$ & 5.38 & 3.50 \\
\hline $\mathrm{NaHCO}_{3}(0.5 \mathrm{M})$ & 7.60 & 3.30 \\
\hline $\mathrm{CaCl}_{2} .2 \mathrm{H}_{2} \mathrm{O}(0.25 \mathrm{M})$ & 5.74 & 7.17 \\
\hline $\mathrm{NaHCO}_{3}(0.25 \mathrm{M})$ & 7.19 & 3.71 \\
\hline $\mathrm{CaCl}_{2} .2 \mathrm{H}_{2} \mathrm{O}(0.1 \mathrm{M})$ & 6.16 & 6.80 \\
\hline $\mathrm{NaHCO}_{3}(0.1 \mathrm{M})$ & 6.90 & 6.28 \\
\hline $\mathrm{Kitano}_{\left(\mathrm{CaCO}_{3} \text { solution}\right)}$ & 6.64 & \\
\hline
\end{tabular}

Table 3.1: The pH of the different mineralization solutions used before and after addition of $2.5 \%$ w/w PAA w.r.t dry weight of chitosan used.

In order to manipulate the degree of mineralization within the 3D scaffold, different mineralization methods were followed. Accordingly, the scaffolds were mineralized using either the McGrath method (alternate soaking + Kitano method) or only the Kitano method over different time periods and solution concentrations. In the case of the McGrath method, the concentration of mineral precursor solution used in the alternate soaking step was 0.5 $\mathrm{M}, 0.25 \mathrm{M}$ or $0.1 \mathrm{M}$ with the soaking time varied between $1 \mathrm{~h}$ to $24 \mathrm{~h}$. The variation in the morphology of the resulting calcium carbonate crystallites was investigated.

\subsubsection{Scanning electron microscopy (SEM) and energy dispersive spectroscopy (EDS)}

SEM was performed to investigate the structural features of the dried chitosan hydrogelbased scaffolds and to determine the morphology of the $\mathrm{CaCO}_{3}$ crystallites formed within the mineralized scaffolds. EDS was performed to determine the elemental composition and also to study the distribution of the minerals within the final mineralized scaffold. For SEM, the sample preparation method and instrument specifications were the same as stated in Section 2.2 of Chapter 2. EDS images were acquired using the same instrument (JEOL JSM$6610 \mathrm{LV}$ ) under backscattered electron image (BEI) mode at $20 \mathrm{kV}$ accelerating voltage with a $10 \mathrm{~mm}$ working distance. 


\subsubsection{Micro-computed tomography (micro-CT):}

Micro-CT was used for 3D visualization of the mineralized 3D chitosan hydrogel-based scaffolds. The processing technique is the same as described in Chapter 2. The setting used to image composites using a Bruker SkyScan1272 high-resolution instrument (N.V., Kontich, Belgium), are a source voltage of $55 \mathrm{kV}$, current of $160 \mu \mathrm{A}$ and an exposure time of $2600 \mathrm{~ms}$. All samples were scanned at a resolution of $2 \mu \mathrm{m}$ and a $0.25 \mathrm{~mm} \mathrm{Al} \mathrm{filter} \mathrm{was}$ applied during imaging. Raw slices were reconstructed to sequential transaxial tomograms using NRecon 1.6.9.18 reconstruction software from Bruker. Beam hardening corrections were applied (92\%), smoothing was set to 4 and the ring artefact correction was set to 16 . Reconstructed data were further processed using CTAn 1.14.4 (Bruker micro-CT) analysis software. The threshold applied for 3D data-set analysis was between 17 to 255 greyscale values. The porosity of the final air-dried chitosan- $\mathrm{CaCO}_{3}$ composite materials was estimated via the same method as described in Chapter 2, Section 2.3.5.

\subsubsection{X-Ray diffraction (XRD)}

XRD was used for compositional analysis as well as to identify the polymorph of the calcium carbonate crystallites in the chitosan- $\mathrm{CaCO}_{3}$ composites. For analysis, the samples were powdered in liquid nitrogen and tested using a PANalytical X'Pert Pro powder diffractometer using $\mathrm{Cu}-\mathrm{K} \alpha$ radiation. The XRD patterns were obtained with generator settings of $45 \mathrm{kV}$ and $40 \mathrm{~mA}$, scans were acquired with $2 \theta$ values from 5 to $70^{\circ}$ at a speed of $0.01 \%$. The resulting diffraction patterns were compared with those of known compounds from the database, using the software X'Pert Highscore $2.2 \mathrm{c}$.

\subsubsection{Thermogravimetric analysis (TGA)}

TGA is usually used to analyse the thermal deposition behaviour of a material by heating it from room temperature up to $900{ }^{\circ} \mathrm{C}$. However, using this technique the percentage mineral composition of the composite scaffold can also be determined. $25 \mathrm{mg}$ of the solid sample to be analysed was placed in the thermogravimetric analyser (Shimadzu TGA-50) and heated to $900{ }^{\circ} \mathrm{C}$ from room temperature at a heating rate of $10^{\circ} \mathrm{C} \mathrm{min}{ }^{-1}$ under a dynamic air atmosphere $\left(50 \mathrm{~mL} \mathrm{~min}^{-1}\right)$. Due to the limitation of the amount of sample that can be analysed in a single run in this instrument, the entire scaffold ( $>25 \mathrm{mg}$ ) was divided into $\leq 25 \mathrm{mg}$ samples and measurements were repeated until the whole scaffold was thermally analysed. 


\subsection{Results and discussion}

\subsubsection{Translating the mineralization achieved in 2D films to 3D scaffolds}

As explained in Chapter 2, the concentration of the chitosan hydrogel was increased from $2 \% \mathrm{w} / \mathrm{v}$ to $5 \% \mathrm{w} / \mathrm{v}$ chitosan (in $2 \%$ acetic acid solution) in order for the gel to be used as the printing ink in our custom designed 3D printer. Although the printability of the ink was improved, the polymer network density was also subsequently increased. Increasing the density in turn affects the extent of penetration of the mineralization media into the $3 \mathrm{D}$ scaffold in comparison with the 2D films (developed using lower concentration of hydrogel) when the same mineralization procedure is used. This meant that some modifications were required to achieve similar growth outcomes.

The method followed for mineralization of 2D chitosan hydrogel scaffolds, as explained in the literature by Evans et al., involves oven drying the wet hydrogel films (opaque) at $37^{\circ} \mathrm{C}$ over a period of $24 \mathrm{~h}$ first (golden in colour and transparent) and then soaking in $1 \mathrm{M} \mathrm{NaOH}$ to achieve enhanced gelation (films now look white and opaque). The film was then washed until neutral $\mathrm{pH}$ prior to being exposed to the McGrath mineralization process [15]. Although the hydrogel undergoes some dimensional shrinkage, due to closure of pores during the oven drying process, it readily swells in the slightly acidic mineralization media, with the concomitant reopening of pores prior to the initiation of the mineralization process, which aids the uniform growth of calcium carbonate crystallites on and within the entire 2D template.

When the same protocol was used to mineralize 3D printed chitosan hydrogel-based scaffolds, the observations were different. Oven drying resulted in shrinkage of the scaffold in similarity to what was described in Chapter 2 for the case of air-dried 3D printed scaffolds. However, unlike for the 2D films, the various steps that followed, such as soaking in $1 \mathrm{M} \mathrm{NaOH}$ for enhanced gelation and use of the McGrath mineralization process, did not result in extensive and uniform swelling of the 3D scaffold. Swelling was observed mostly on the exterior surfaces of the scaffold which was in immediate contact with the various solutions. The swollen regions were easily distinguished from the rest of the scaffold. These regions appeared opaque compared to the transparent golden yellow colour of the dry nonswollen regions, where the solutions had not readily penetrated. Figure 3.2 shows optical images of the 3D chitosan hydrogel-based scaffolds at various stages before and after 
mineralization via the method used previously by the McGrath group to mineralize 2D chitosan hydrogel-based templates. Herein, the non-swollen regions are shown within the dotted red line. Table 3.2 depicts the change in the diameter of the scaffold during the same.
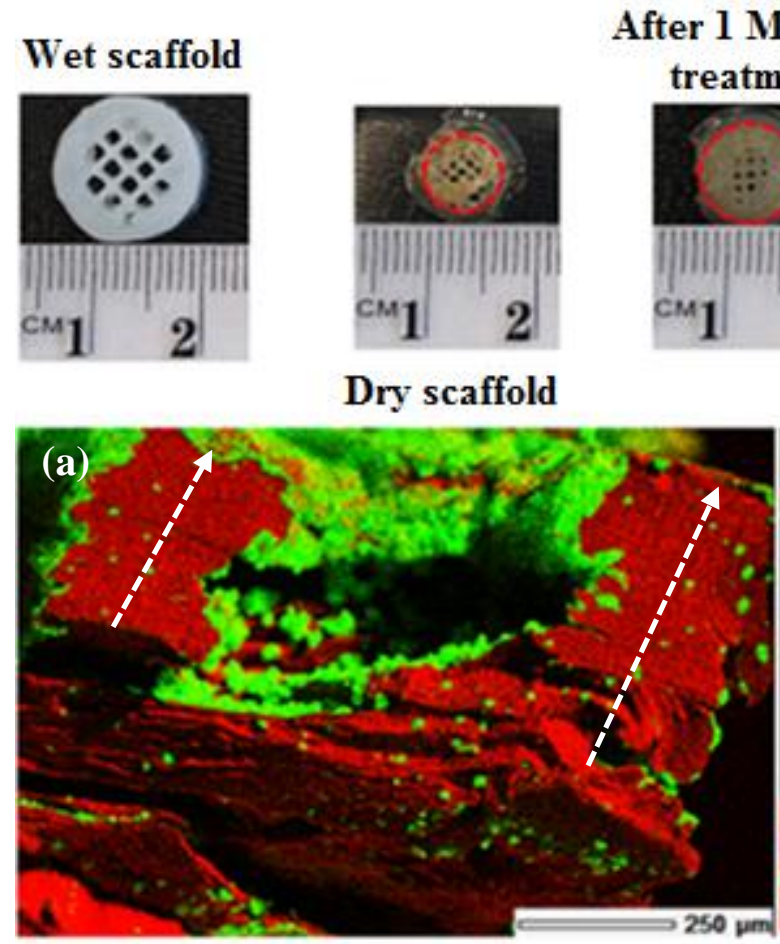

After $1 \mathrm{M} \mathrm{NaOH}$
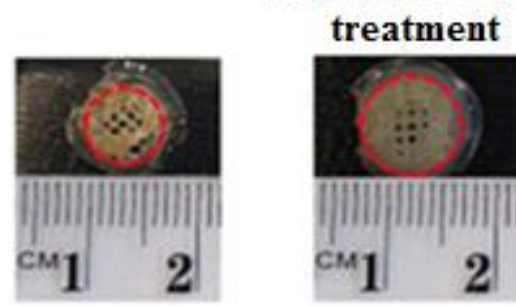

Dry scaffold

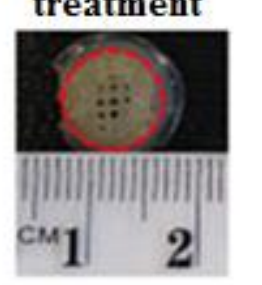

2

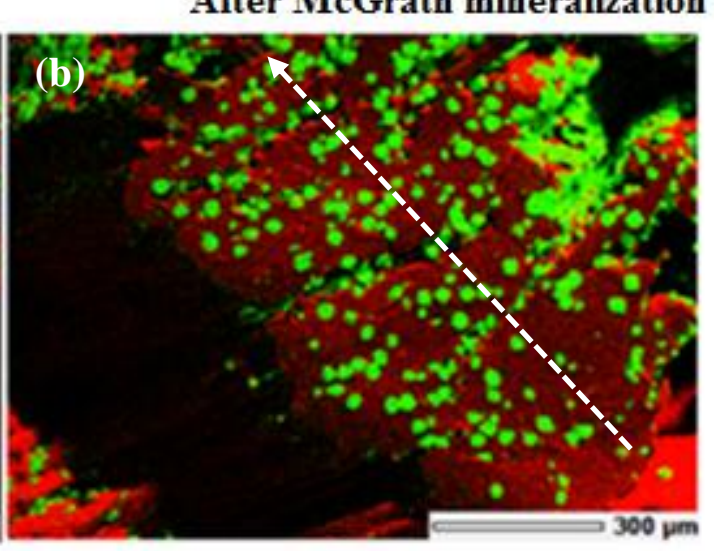

Final composite

(dried)
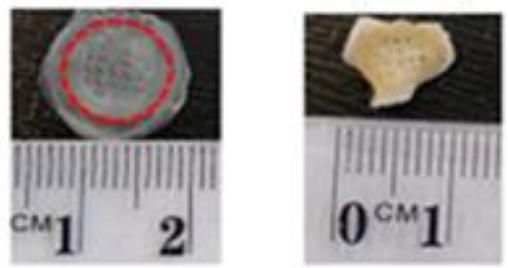

After McGrath mineralization

Figure 3.2: Top row: optical images of 3D printed chitosan hydrogel-based scaffolds at various stages before and after mineralization according to the processing method used for $2 D$ chitosan hydrogel-based templates. The regions encircled in red dashes represent the regions that did not show any swelling during the process and remained slightly golden in colour. Bottom row: EDS images of the cross-sections (in the $Z$ direction marked by white arrow) of the final composites prepared via the processing method used for (a) $2 D$ films and $(b)$ the modified protocol developed in this work. Green regions correspond to Ca and red is $C$.

Although appreciable swelling was observed in the scaffolds when using the mineralization method followed for 2D templates, EDS images (Figure 3.2 (a)) confirm that mineralization was not as extensive as in the case in the scaffolds mineralized using a modified method (Figure 3.2 (b)). EDS images of the cross-section (along the height (Z-axis)) of the mineralized 3D scaffold showed that, in comparison to the exterior surface of the scaffold, the mineralization achieved in the interiors (regions that are not directly exposed to the mineralization media) was significantly less. The limited mineralization observed in the inner regions of the scaffold may be due to non-uniform swelling of the dry scaffold and the 
resultant lack of porous channels that could facilitate the extensive diffusion of the mineralization media into its interiors.

\begin{tabular}{|c|c|c|c|}
\hline $\begin{array}{c}\text { Initial wet } \\
\text { state (mm) }\end{array}$ & $\begin{array}{c}\text { After oven } \\
\text { drying (mm) }\end{array}$ & $\begin{array}{c}\text { After gelation } \\
\text { in } \mathbf{~} \mathbf{~ M ~ N a O H} \\
(\mathbf{m m})\end{array}$ & $\begin{array}{c}\text { After undergoing mineralization } \\
\text { via the McGrath method - wet }\end{array}$ \\
\hline 14 & 6 & 8 & 10 \\
\hline
\end{tabular}

Table 3.2: The variation in the diameter of a 3D printed chitosan hydrogel-based scaffold in the wet state prior to and during the various stages of the mineralization process as used for $2 D$ films. The diameter is calculated based on the regions encircled in red dashes as shown in Figure 3.2 (top), this region is considered for analysis as only the chitosan hydrogel layers in this region showed the same dimensional shrinkage upon air-drying compared to the rest of scaffold and should hence show comparable swelling.

Although the 2D films and 3D chitosan hydrogel-based scaffolds are essentially the same in most attributes, the polymer network density in the case of the dried 3D scaffolds is much higher than for the 2D films (even when the same concentration of polymer used to develop these systems). This has consequences with respect to how each of these systems interacts with solvents. The effect is further enhanced in the case when the higher concentration of polymer used to develop 3D systems and also due to the layer-by-layer stacking during the fabrication process when compared to that of the thin 2D films fabricated using $2 \% \mathrm{w} / \mathrm{v}$ chitosan hydrogel. This increased polymer network density restricts the extent of diffusion and also therefore restricts the extent of swelling occurring within 3D scaffold when soaked in the mineralization media, which in turn restricts the diffusion and thus the extent of mineralization throughout the 3D scaffold. Omidian et al. explains that this kind of restricted solubility and hence lower swelling of the polymer chains results from higher elastic forces being present at the polymer chain crosslinks, which in the case of 3D structures may be stronger due to stacking of layers of physically crosslinked hydrogel as is the case of a 3D scaffold [19].

Accordingly, the pre-mineralization treatment for 3D chitosan hydrogel-based scaffolds was modified to eliminate the oven drying step prior to soaking in $1 \mathrm{M} \mathrm{NaOH}$. This ensures that, when the $\mathrm{pH}$ neutralized 3D scaffolds are introduced into the mineralization media, the inherent porous nature of the hydrogel has been maintained, hence facilitating maximum 
diffusion of ions into the interior of the scaffolds. Figure 3.2 (b) shows the EDS images of scaffolds mineralized via the modified processing technique wherein, an increased signal was observed for $\mathrm{Ca}$ in the cross-section of the mineralized 3D chitosan hydrogel-based scaffold. This shows that the extensive porous network maintained within the 3D scaffold owing to the elimination of the oven drying process (as followed in the case of 2D templates), prior to mineralization leads to the diffusion of mineralization media into the interiors of the scaffolds as well, which thereby results in increasing the extent of mineralization throughout the scaffold (both on the exterior surface and the interiors of the 3D scaffold).

\subsubsection{Mineralization of 3D chitosan hydrogel-based scaffolds}

Using the modified McGrath method (hereafter referred to as the McGrath method for simplicity) appreciable mineralization was achieved in 3D chitosan hydrogel-based scaffolds. SEM analysis revealed that the mineralization process did not lead to clogging of the printed macropores although a good amount of calcium carbonate crystallites was observed to be growing on the walls of the macropores which were in contact with the mineralization media (Figure 3.3 (a)). Such growth will slightly reduce the average pore size and porosity of the final dried composites compared to those of the dried nonmineralized chitosan hydrogel-based scaffolds (discussed later in Section 3.3.7).

Extensive mineralization was observed in regions of the scaffold in contact with the mineralization solution (Figure 3.3 (b)), e.g. the exterior surface of the scaffold. SEM images of the cross-sections parallel and perpendicular to the height of the scaffold revealed that extensive mineralization was achieved in between the printed layers as well (refer to EDS images in Figures 3.7 and 3.16).

The McGrath method was developed as a biomimetic technique in an effort to replicate the elements of the nanostructure of nacre in the laboratory. Mimicking the nanostructure of nacre requires the presence of an insoluble polymer matrix in close contact with a moderately concentrated precursor ion solution and crystal growth modifiers such as certain acidic proteins [20]. Here the chitosan hydrogel-based scaffold acts as the insoluble polymer matrix facilitating heterogeneous nucleation of $\mathrm{CaCO}_{3}$ molecules from the mineralization media. The McGrath mineralization method, which is a combination of alternate soaking and Kitano method, ensures the presence of moderately concentrated mineral precursors throughout the hydrogel scaffold, facilitating the growth of calcium carbonate crystallites 
within the hydrogel matrix. The mineralization process is driven by a diffusion dependent heterogeneous nucleation process resulting in the growth of $\mathrm{CaCO}_{3}$ crystallites within the hydrogel matrix. During the alternate soaking step, diffusion and electrostatic attraction driven mechanisms ensure the availability of reactive mineral ions/precursors $\left(\mathrm{Ca}^{2+}\right.$ and $\mathrm{HCO}_{3}{ }^{-}$) within the porous 3D hydrogel matrix. The resulting clusters of $\mathrm{CaCO}_{3}$ molecules act as seeds/nuclei within the matrix which further grow in the presence of saturated $\mathrm{CaCO}_{3} /$ Kitano solution leading to the extensive mineralization observed throughout the scaffold.

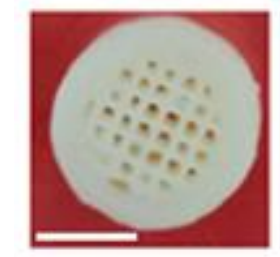

(a)
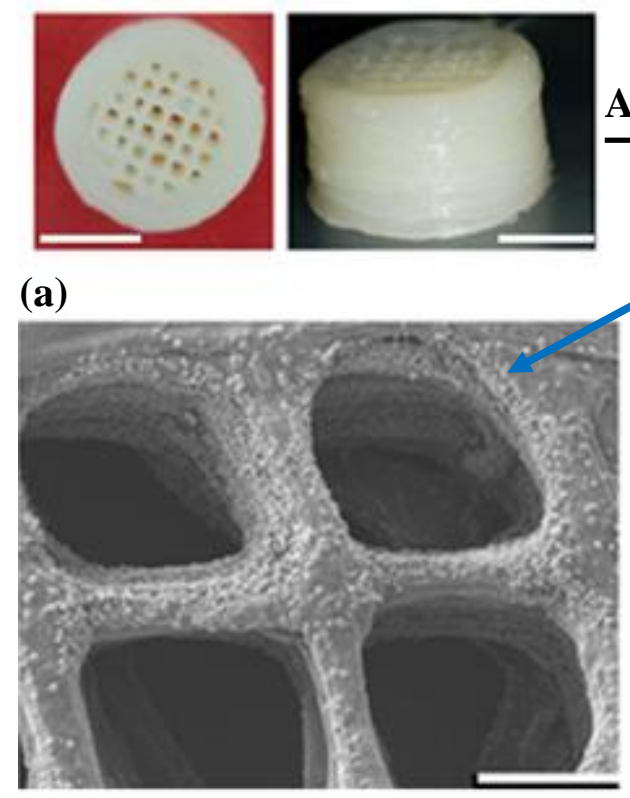

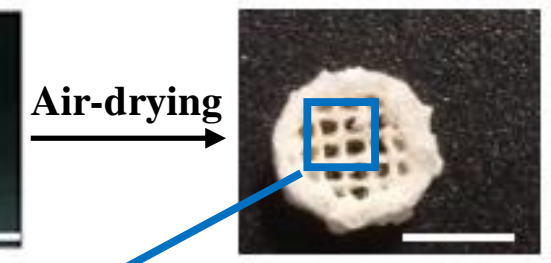

(b)

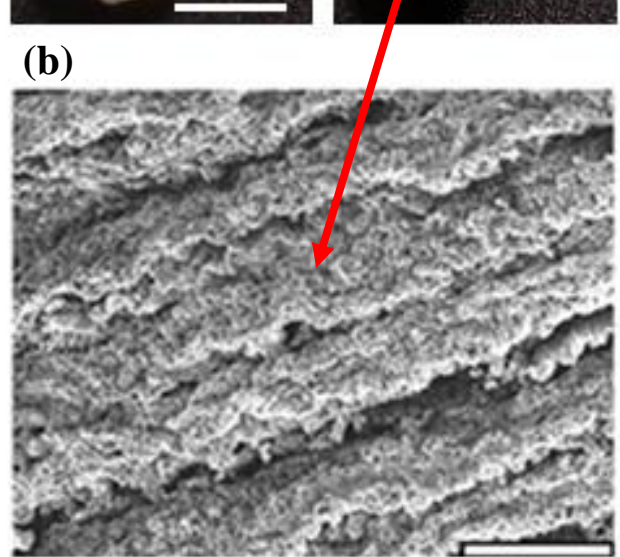

Figure 3.3: Optical images of chitosan hydrogel-based scaffolds mineralized via the McGrath method before (top left) and after (top right) air-drying. The SEM images of the air-dried mineralized scaffolds showing the mineral growth observed (a) on the macropores walls; and (b) the external cylindrical region of the scaffold which was in contact with the media during the mineralization process, individual printed layers are clearly visible. The scale bar of the optical images is $0.5 \mathrm{~cm}$ and that of SEM images is $50 \mu \mathrm{m}$.

Based on the modified mineralization protocol, the 3D chitosan hydrogel-based scaffolds were mineralized when in the wet state via the McGrath method and hence the intrinsic nano and microporous network within the wet hydrogel matrix (as shown in Chapter 2) facilitates enhanced diffusion of the mineralization solution throughout the interiors of the scaffold, hence resulting in good mineralization throughout the scaffold including in between the printed layers of the 3D scaffold. The morphologies of the crystals observed across the different layers of the scaffolds are mostly a combination of rhombohedra, rosettes, and 
spherical or stacked platelet-like structures. Such morphologies are traditionally observed when precipitating $\mathrm{CaCO}_{3}$ in solution in the absence of crystal growth modifiers. Figure 3.4 depicts different $\mathrm{CaCO}_{3}$ crystal morphologies obtained as a result of the mineralization of 3D chitosan hydrogel-based scaffolds via the McGrath method in the absence of crystal growth modifiers.
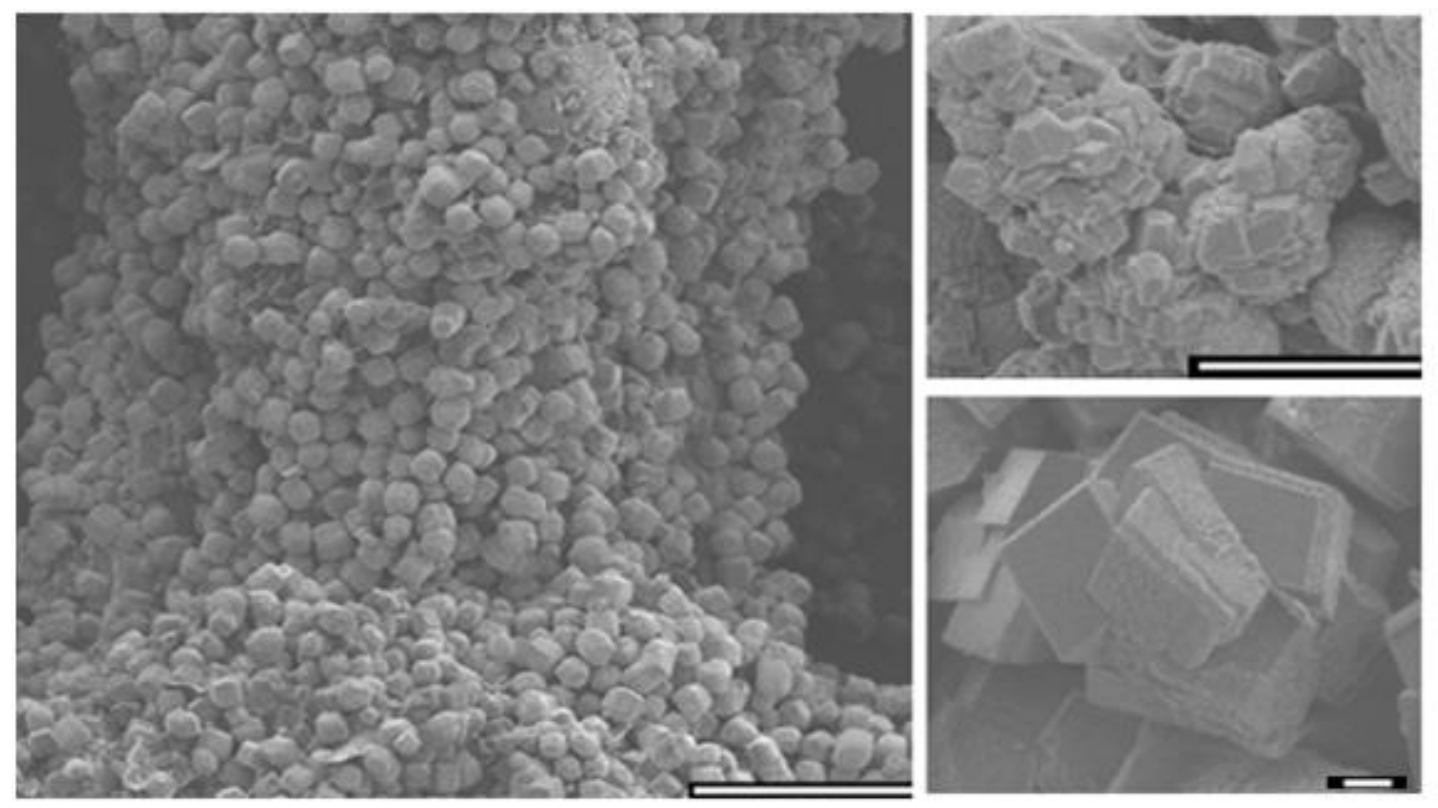

Figure 3.4: SEM images of a 3D chitosan hydrogel-based scaffold mineralized via the McGrath method in the absence of crystal growth modifiers, the image on the left is the mineralization observed on a strut of the $3 D$ scaffold directly exposed to the mineralization media. On the right are higher magnification images showing different morphologies of the resultant $\mathrm{CaCO}_{3}$ crystallites formed. Scale bar for the image on the left is 100 um and for those on the right is $10 \mu \mathrm{m}$.

Literature suggests that the morphology of $\mathrm{CaCO}_{3}$ crystallites formed in the presence of a hydrogel matrix can be modified using certain crystal growth modifiers and that when such additives are associated with the hydrogel matrix, this facilitates crystallite nucleation in the matrix, suppressing crystal nucleation in solution [21]. The mineralization of 2D chitosan hydrogel films via the McGrath method in the presence of polyacrylic acid (PAA) as the crystal growth modifier has shown that PAA interacts with the growing $\mathrm{CaCO}_{3}$ crystallites resulting in a morphology similar to that of nacre in its early stages of development as shown in Figure 3.1 [22-24]. A similar mineral growth was observed when using 3D chitosan hydrogel-based scaffolds as well. In the case of 3D systems, it was observed that the crystal morphology is modified from the traditional calcite structures to aggregates of nanocrystals 
growing laterally to the polymer network, in the form of flat pancake-like structures (Figure 3.5 (b)) (when mineralized in the presence of at low concentration of precursor mineral ion solutions). The literature shows that this is due to the suppressed axial growth of the growing crystallites due to their interaction with PAA, hence promoting lateral growth [25, 26]. SEM images revealed that good mineral growth was achieved throughout the regions in direct contact with the mineralization media, in between the layers of the scaffold and the exposed regions of the printed macropores (Figure 3.5 (a)). Similar to the case of scaffolds mineralized in the absence of PAA, the printed macropores were not clogged, when mineralized in the presence of PAA.
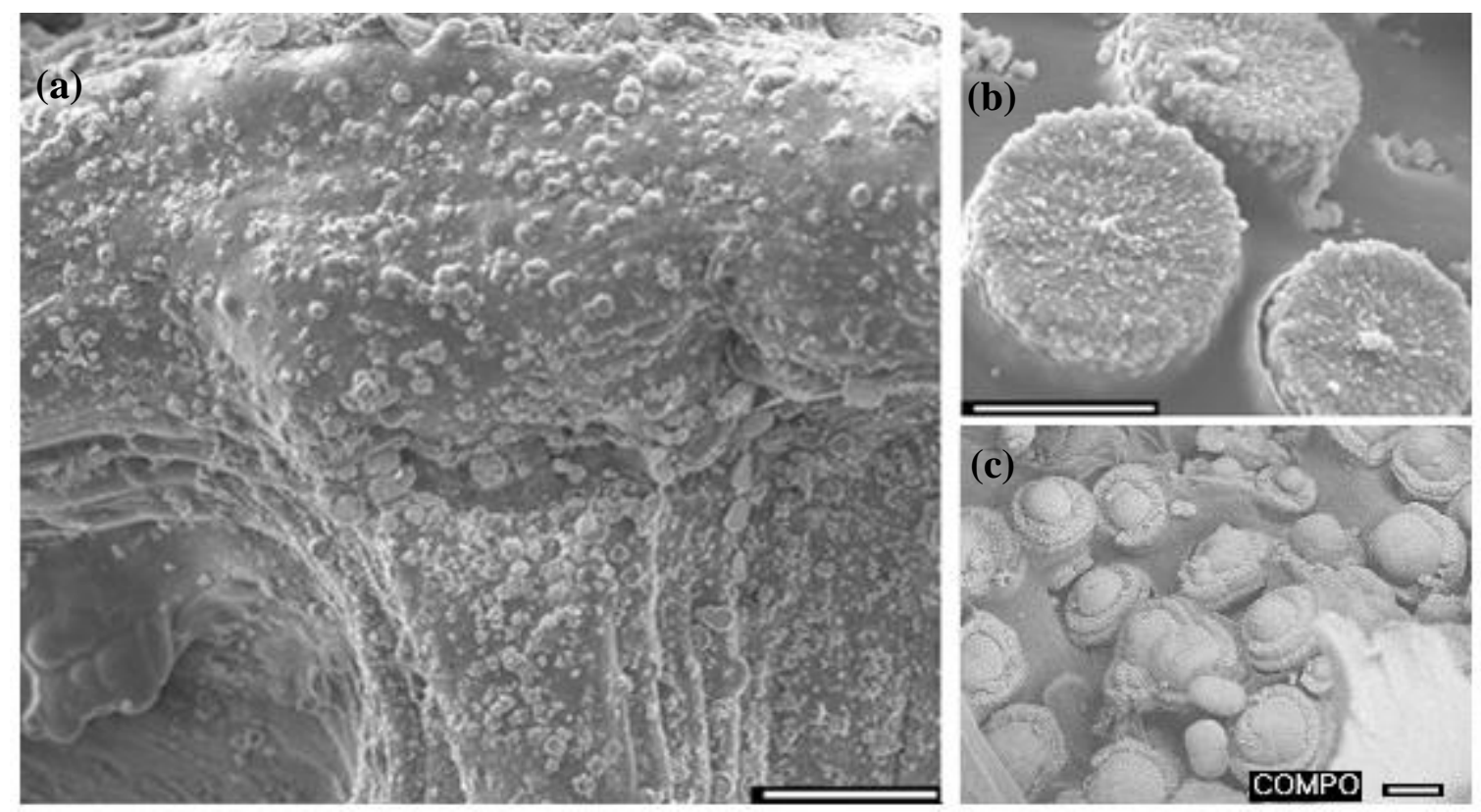

Figure 3.5: SEM images of 3D chitosan hydrogel-based scaffolds mineralized via the McGrath method in the presence of PAA. (a) Mineralization observed at the nodes (regions where struts overlap) of the 3D scaffold directly in contact with the mineralization media; (b) pancake-like $\mathrm{CaCO}_{3}$ crystallites; and (c) pancake-like crystallites with globular crystallites growing on their top surface. Scale barfor the image on the left is $100 \mu$ mand for those on the right is $10 \mu \mathrm{m}$.

The preferred crystallite morphology, when McGrath mineralization is performed in the presence of PAA, is the one that closely imitates the platelet-like morphology of $\mathrm{CaCO}_{3}$ in nacre i.e. the flat pancake-like structure. It should be noted that other morphologies were also observed as the mineralization protocol was varied. When precursor ion solutions concentrations as high as $0.5 \mathrm{M} \mathrm{Ca}^{2+}$ and $\mathrm{CO}_{3}{ }^{2-}$ were used during the alternate soaking step (followed by Kitano soaking), the resulting crystallites assumed a morphology as shown in 
Figure 3.5 (c). In this case a secondary growth, in the form of spherical structures growing on the surface of the pancake-like crystallites occurred. Since this work is directed towards replicating nacre-like nanostructures in a 3D chitosan hydrogel-based system, various strategies adopted to reduce the appearance of such irregular morphologies, are explored in later sections of this chapter.

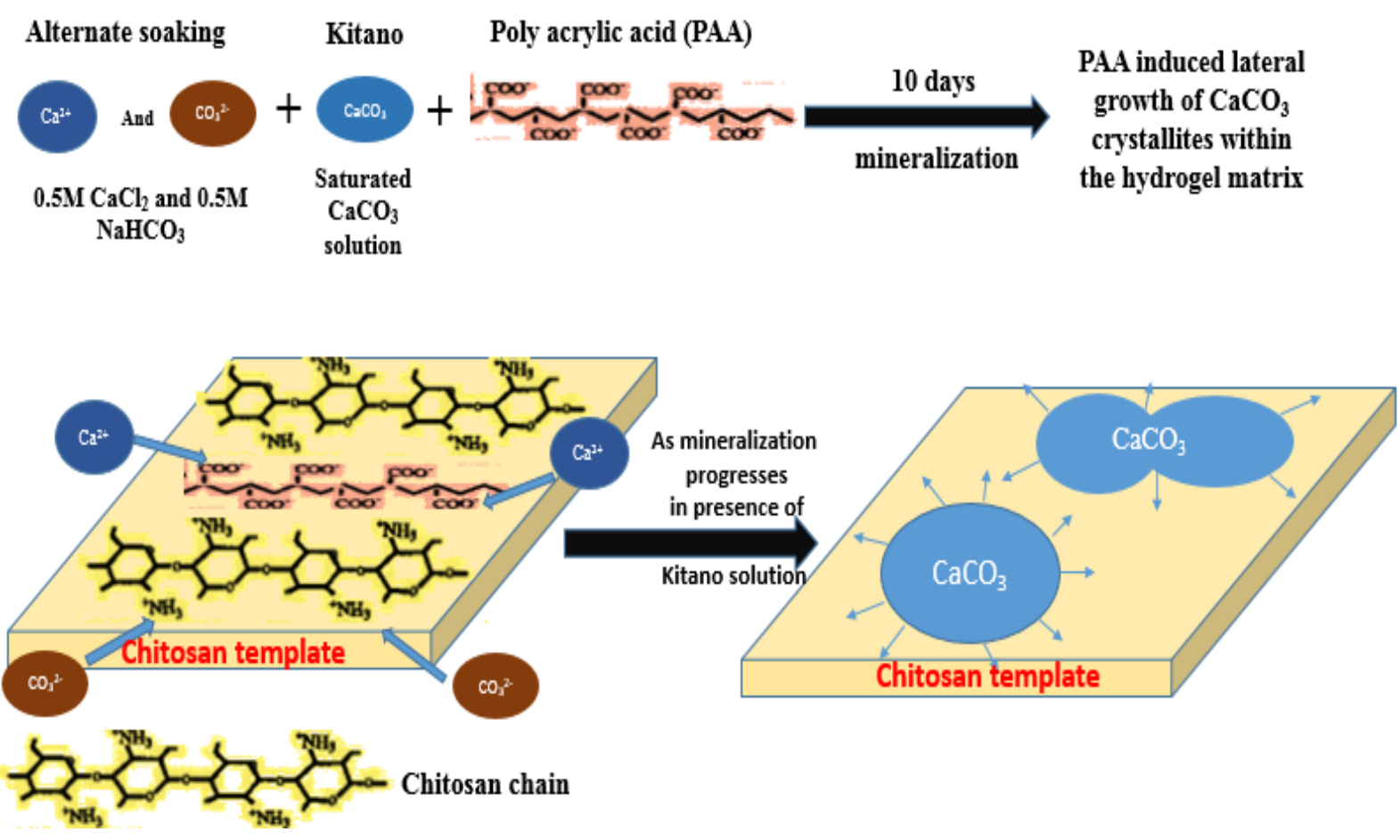

Figure 3.6: Schematic representation of the mineralization achieved on a $2 D$ chitosan hydrogel-based template in the presence of PAA. Adapted from [27] with permission.

The interaction between PAA and chitosan hydrogel is electrostatic in nature, arising from the interaction between the dissociated carboxylic groups of PAA and the protonated amino groups of chitosan. This results in the formation of a polyelectrolyte complex [28]. When 3D chitosan hydrogel-based scaffolds are mineralized via the McGrath method in the presence of PAA, the polyelectrolyte complex thus formed with the scaffold matrix reduces the free energy of the system and the sites where the complex is present become the most suitable site for nucleation of $\mathrm{CaCO}_{3}$ molecules from the mineralization solution. Upon addition of mineral precursors (calcium chloride and sodium hydrogen carbonate), the free $\mathrm{COO}^{-}$groups of PAA attracts $\mathrm{Ca}^{2+}$ ions, this creates a localized increase in positive ions which further leads to the attraction of $\mathrm{CO}_{3}{ }^{-}$ions. These reacting ions are at sufficient concentrations to initiate seed formation [29]. As the mineralization continues in the presence of saturated $\mathrm{CaCO}_{3} / \mathrm{Kitano}$ solution, PAA also acts as a crystal growth inhibitor 
restricting the growth of $\mathrm{CaCO}_{3}$ crystallites along certain planes. The resulting crystallite grows laterally forming flat pancake-like structures growing parallel to the matrix. Hence, the lateral growth of the crystallites is a combined action of both the insoluble organic matrix and the acidic polymer additive [10]. This theory is supported by SEM images taken at lower length scales showing that when mineralization is performed in the presence of PAA, the crystallites formed were closely associated (entangled in the hydrogel matrix) with the hydrogel matrix (Figure 3.8; explored later in this section). A schematic representation of the proposed interaction between chitosan hydrogel-PAA- $\mathrm{CaCO}_{3}$ is shown in Figure 3.6 (adapted from [30]).

In the SEM images of scaffolds mineralized in the absence of PAA, no evidence of such an interaction was observed between the crystallites and the chitosan hydrogel matrix. In this case, since a polyelectrolyte complex is not formed, the chitosan hydrogel scaffold merely acts as a low energy surface facilitating nucleation of the crystallites similar to the nucleation happening on any other surface in contact with the mineralization media. This is in accordance with studies reported in the literature [31]. The interaction between the PAA, the chitosan polymer matrix and the $\mathrm{CaCO}_{3}$ crystallites is assumed to be similar to the association of acidic macromolecules with growing calcium carbonate crystallites during the nacre-biomineralization process which results in the formation of single crystal aragonite $\mathrm{CaCO}_{3}$ platelets. These acidic macromolecules are also said to play a role in maintaining the thermodynamic stability of calcium carbonate single crystals existing as the less stable aragonite polymorph within nacre [30].

EDS analysis of mineralized scaffolds after air-drying confirmed the presence of $\mathrm{CaCO}_{3}$ crystallites in the 3D hydrogel matrix (Figure 3.7). In the regions of the scaffold directly exposed to mineralization media (at certain concentrations of mineralization solution) the crystallites were seen to grow laterally mostly as pancake-like structures growing on the hydrogel matrix. Also, in the SEM images of some regions (directly exposed to the mineralization media and in between the layers), certain protrusions were observed in the organic matrix. An EDS map of these regions (protrusions) showed high-intensity signals for $\mathrm{Ca}$ and $\mathrm{C}$, suggesting that these protrusions were in-effect $\mathrm{CaCO}_{3}$ crystallites growing within the matrix and protruding further outwards (the encircled region in Figure 3.6). This further supports the fact that the extensive network of pores within the wet chitosan hydrogel matrix facilitates diffusion of the mineralization media across the entire scaffold when the mineralization is performed using scaffolds in their wet condition. Hence, facilitating 
nucleation of $\mathrm{CaCO}_{3}$ crystallites within the nano and micropores (present in the wet chitosan hydrogel scaffolds) and further growing out of the porous matrix as the crystallites accumulate during growth.

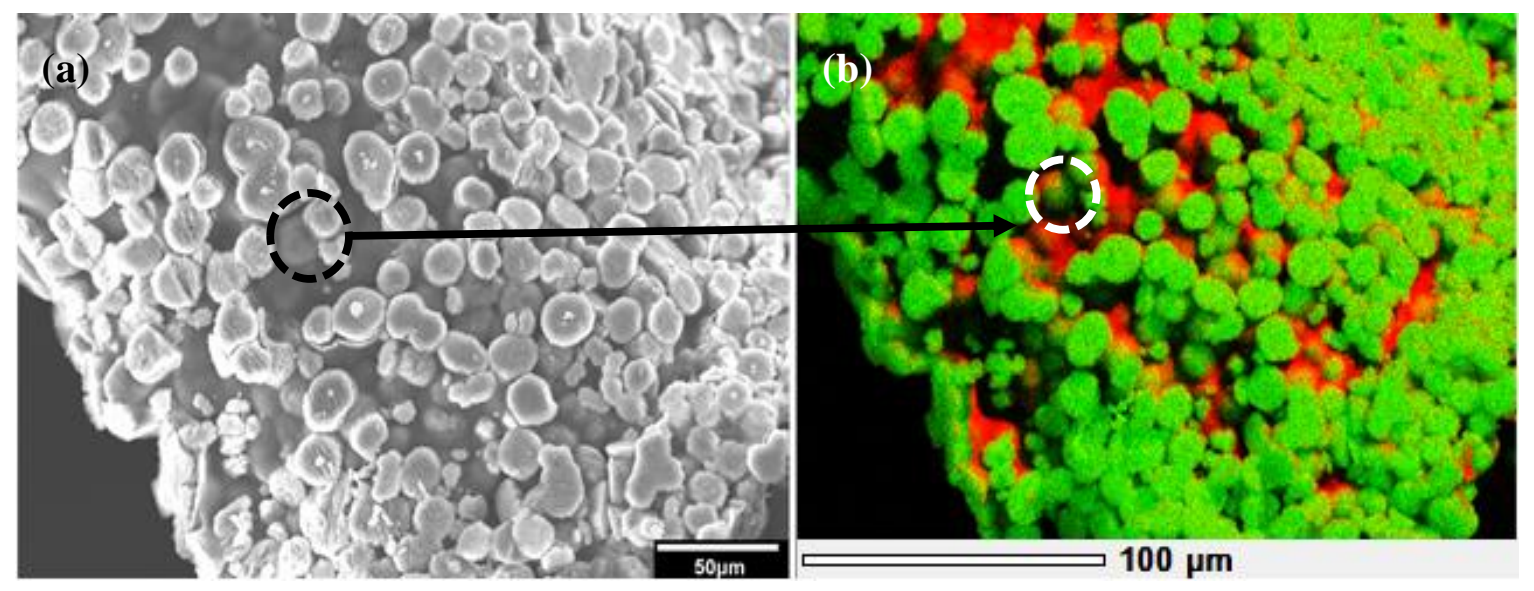

Figure 3.7: 3D chitosan hydrogel-based scaffold mineralized via the McGrath method in the presence of PAA showing (a) an SEM image of a printed layer of the scaffold exposed directly to the mineralization media and (b) its corresponding EDS map showing the calcium carbonate platelets (Ca is shown in green) growing evenly on the scaffold surface ( $\mathrm{C}$ shown in red). The dotted region corresponds to $\mathrm{CaCO}_{3}$ crystallites protruding out hydrogel matrix that is being picked up in the EDS maps as a slight green patch. The samples were prepared by fracturing air-dried mineralized $3 D$ scaffolds after freezing in liquid nitrogen.

At a higher resolution, it is observed that each pancake-like crystallite (observed in the regions of the scaffold directly exposed to the mineralization media) is actually a cluster of several $\mathrm{CaCO}_{3}$ nanoplatelets, growing in association with the hydrogel matrix (Figure 3.8). During the initial stages of crystal formation in nacre biomineralization, it has been shown that individual $\mathrm{CaCO}_{3}$ nanoparticles assemble into larger platelet mesocrystals, stacking along the same crystal orientation within individual platelets. Over time, due to the high adhesion of the mesocrystals, they integrate, and the grain boundaries vanish resulting in a single crystal aragonite platelet $[32,33]$. The images shown in Figure 3.8 suggests a similar situation wherein, individual nanosized platelet-like crystallites seemingly organize themselves into regularly oriented clusters similar to mesocrystal-like structures within a microscopic pancake-like crystallite. However, no analysis was performed to confirm this hypothesis. 


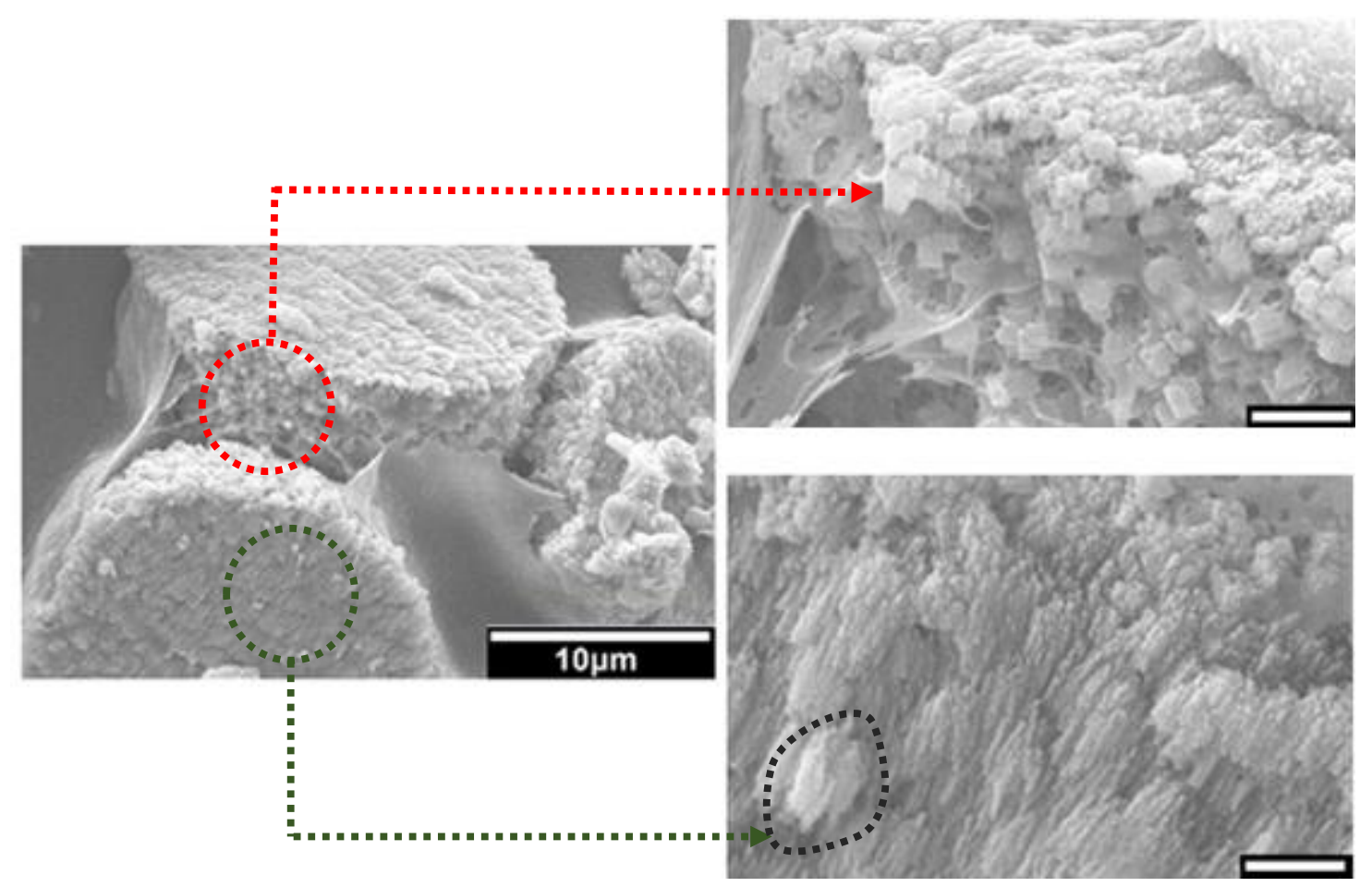

Figure 3.8: SEM images of the top surface and lateral view of pancake-like calcium carbonate crystallites formed in the $3 D$ chitosan hydrogel matrix when mineralized using the McGrath method in the presence of PAA. The black dotted lines show a cluster of $\mathrm{CaCO}_{3}$ platelets that are assumed to be similar to a mesocrystal-like structure. Scale bar of the images on the right is $1 \mu \mathrm{m}$.

Mesocrystals are defined as aggregates of iso-oriented nanocrystals which are initially separated by organic matrix or solvent that then fuse to form single crystals. The formation of mesocrystals is also evidence for the active involvement of the hydrogel matrix in the heterogeneous nucleation of $\mathrm{CaCO}_{3}$ crystallites [34]. Song et al. proposed that the plausible mechanism for the formation of mesocrystals is either by filling the porous hydrogel matrix compartments with nanoparticles or alignment of the nanoplatelets along the oriented matrix. These nanoparticles could either be generated first and then be oriented with the organic matrix or nucleate on the organic matrix (a possible phenomenon occurring when the McGrath method is used), and then undergo oriented aggregation. Owing to the formation of a polyelectrolyte complex between the chitosan hydrogel matrix and the PAA, the latter phenomenon is more likely to be favoured than the former when anionic crystal growth modifiers like PAA are present. In the micron-sized pancake-like crystallites, oriented nanoplatelets are seen stacked parallel to each other and perpendicular to the matrix surface. Each nanoplatelet within the pancake-like crystallite is intricately associated with 
the organic matrix, as is evident in Figure 3.8. Unlike the single crystals found in mature nacre, wherein the mesocrystals have fused and grain boundaries have vanished, in our 3D scaffolds, the clustered nanoplatelets create a textured (roughness) appearance on the surface of pancake-like crystallites.

Starting with the nucleation of $\mathrm{CaCO}_{3}$ crystallites within the nano-microscale pores of the chitosan hydrogel, the nucleates then grow to form nanoplatelets which then assemble to form the micron-sized pancake-like structures; it could be said that the observed individual micron-sized pancake-like $\mathrm{CaCO}_{3}$ crystallites are evidence for hierarchical organization of crystallites in association with the hydrogel matrix. On a macroscale, the mineralized 3D printed chitosan hydrogel-based scaffolds $(0.1 \mathrm{~mm}$ thickness in the wet state $)$ can be visualised as a stack of thin layers of organic matrix interlacing micron-sized $\mathrm{CaCO}_{3}$ crystallites. Hence, replicating the "mortar-brick" architecture of nacre on a higher length scale.
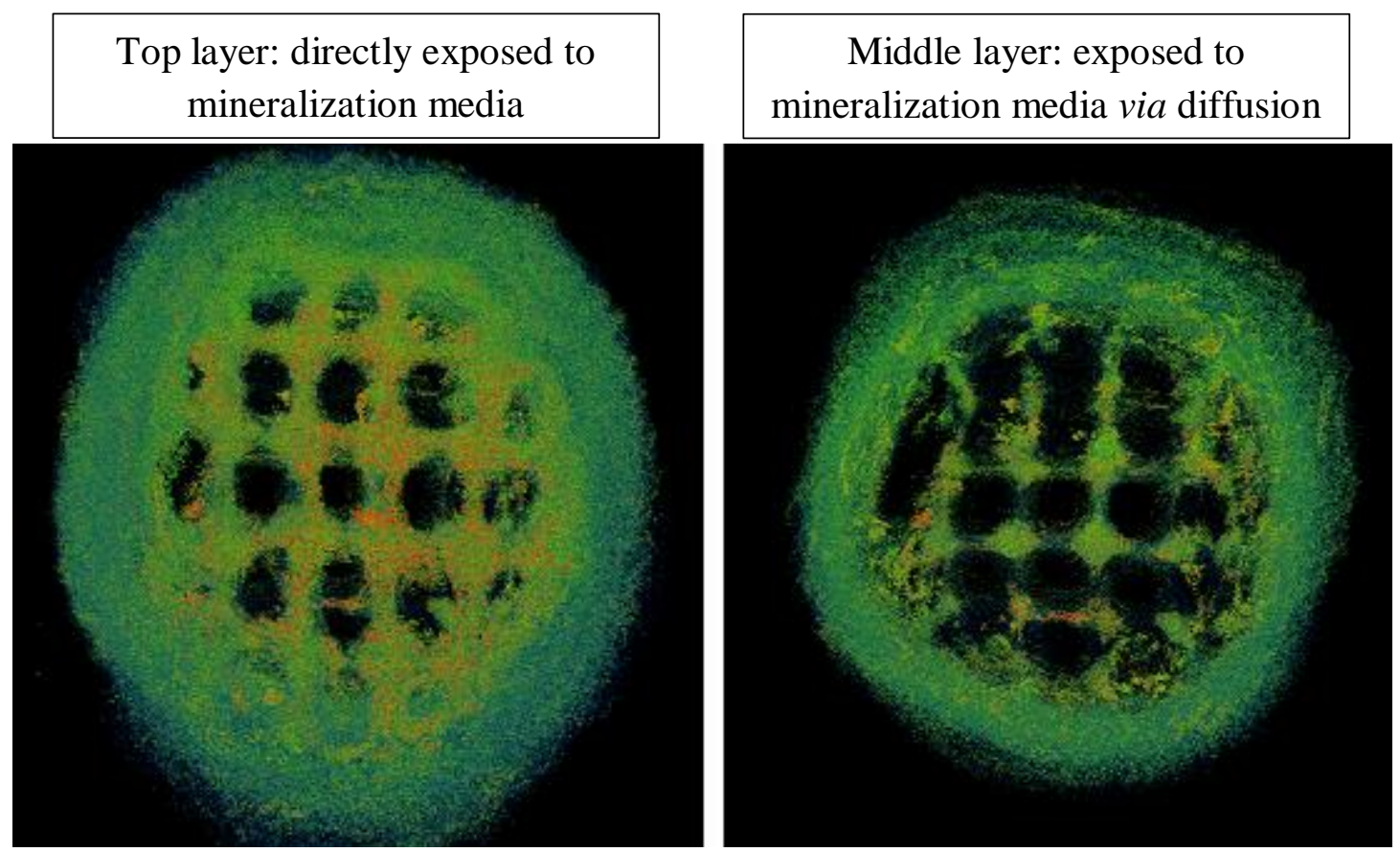

Figure 3.9: A 3D visualization of the reconstructed micro-CT scans of chitosan hydrogelbased scaffold mineralized via the McGrath method in the presence of PAA (alternate soaking in $0.5 \mathrm{M}$ precursor ions solution +7 days Kitano). On the left is the first layer of the scaffold which was directly exposed to the mineralization media and the right is a midsection layer of the scaffold which was not directly exposed to the mineralization media. Blue represents the low-density regions of the scaffold (chitosan) and the rest represents the mineral component of the scaffold, the intense red colour represents the regions with very high mineral content. 
In general, the extent of mineralization within the 3D scaffold was higher in the regions directly exposed to the mineralization media in comparison to in between the printed layers. This was evident in the micro-CT scans of mineralized chitosan hydrogel-based scaffold. Figure 3.9 shows a 3D visualization of the reconstructed micro-CT scans of chitosan hydrogel-based scaffolds mineralized via the McGrath method.

As observed in the SEM images and micro-CT analysis, it is important to note that when using the McGrath mineralization method, the extent of mineralization and the morphology of the $\mathrm{CaCO}_{3}$ crystallites formed is not uniform across the entire 3D scaffold. In particular, the growth in between the layers of the scaffold is different from that which occurs on the outer surfaces of the scaffold that is in direct contact with the mineralization media. There could be many factors contributing to this variation in the morphology such as, the concentration of the precursor ions the scaffold is exposed to during the alternate soaking step, the concentration of the PAA interacting with the precursor ions and the porosity of the scaffold which affects the extent of diffusion of the mineralization media into the scaffold, among other things. To analyse this variation the following were investigated:

(a) the effect of increasing the PAA concentration at a fixed concentration of precursor ions $\left(\mathrm{Ca}^{2+}\right.$ and $\mathrm{HCO}_{3}{ }^{-}$ion solutions);

(b) the effect of increasing the concentration of the precursor ions at a fixed concentration of PAA; and

(c) the effect of the size of the macropores on the extent of mineralization across the scaffold.

\subsubsection{PAA concentration optimization}

As the morphology and distribution of crystallites within the polymer matrix affects the physical properties of the final composite, such as its hardness or compressive strength, it is desirable to achieve a homogenous distribution of $\mathrm{CaCO}_{3}$ of the preferred morphology throughout the entire scaffold. In composites, it has been found that flat platelet-like inclusions contribute to a higher mechanical stability under compressive and tensile stress compared to particle-like inclusions [35]. Hence, it is important to identify the PAA concentration range that results in the most uniform distribution of flat pancake-like crystallites throughout the 3D scaffold, including in between the layers of the scaffold. 
The concentration of PAA was varied from $0.5-3.5 \%$ w/w (w.r.t the dry weight of the chitosan scaffold used) at a constant precursor ion concentration of $0.5 \mathrm{M} \mathrm{Ca}^{2+}$ and $\mathrm{HCO}_{3}{ }^{-}$ ion solutions (alternate soaking solutions). Since the mineralization is performed while the scaffold is in its wet state, the dry weight of chitosan scaffold used is measured by weighing a representative air-dried 3D printed chitosan hydrogel-based scaffold with the same dimensions in the wet state. It is assumed that 3D chitosan hydrogel-based scaffolds fabricated via 3D printing using the same printer, ink parameters and CAD model will have a relatively comparable mass. Based on the dimensions of the wet scaffold, this value is then used to calculate the relative amount of PAA to be added to the mineralization media. The wet scaffolds were soaked alternatively in the precursor ions solutions each for $1 \mathrm{~h}$ and then soaked in Kitano solution for 7 days. The different morphologies observed are summarized in Table 3.3.

Figure 3.10 shows the SEM images of 3D chitosan hydrogel-based scaffolds mineralized via the McGrath method in the presence of different PAA concentrations w.r.t the dry weight of chitosan used. After mineralization, scaffolds were delaminated before air-drying, in order to observe the mineral growth in between the layers in comparison to those in the regions directly exposed to the mineralization media. This was done in the wet state when it is easier to delaminate the scaffolds rather than in its dried state.

It is evident that at each PAA concentration considerable mineralization occurs in between the layers in all samples due to the high concentration of precursor ion solution used. This high concentration was used in order to ensure that the flux of the precursor ions was not a limiting factor of the experiment in order to specifically probe the effect of the concentration of PAA. In the regions that were in direct contact with the mineralization media, the increasing concentration of PAA resulted in well-formed pancake-like crystallites and the effect was more prominent at concentrations above $1.5 \% \mathrm{w} / \mathrm{w}$.

It is important to note that $\mathrm{CaCO}_{3}$ crystal growth kinetics in a hydrogel matrix can easily be altered by very small variations of the concentration of the reactants and properties of the hydrogel matrix such as the variation in the degree of deacetylation of the polymer chains, chain entanglements and swelling behaviour etc. resulting in a multitude of crystallite morphologies [36-38]. Also, since the 3D printing process is semi-automated, there could be small structural defects within the scaffolds which in turn cause the development of pockets of higher concentration of reactants resulting in a morphology different from the 
other regions. Most of these variables cannot be completely optimized. Nonetheless, amongst all the controllable variables, the concentration of PAA chosen is merely a starting point to further research on optimizing the crystallite morphology.

\begin{tabular}{|c|c|c|}
\hline \multirow{2}{*}{$\begin{array}{c}\% \text { PAA } \\
\text { concentration } \\
\text { w/w w.r.t dry } \\
\text { weight of } \\
\text { chitosan }\end{array}$} & \multicolumn{2}{|c|}{$\mathrm{CaCO}_{3}$ morphologies } \\
\hline & On the exterior surface & In between the layers \\
\hline 0.5 & Globular crystallites & $\begin{array}{l}\mathrm{CaCO}_{3} \text { crystallites similar to } \\
\text { rhombohedra }\end{array}$ \\
\hline 1.0 & $\begin{array}{l}\text { Globular crystallites and some } \\
\text { pancake-like crystallites }\end{array}$ & $\begin{array}{l}\text { Aggregates of numerous nanosized } \\
\mathrm{CaCO}_{3} \text { crystallites }\end{array}$ \\
\hline 1.5 & $\begin{array}{l}\text { Pancake-like crystallites with } \\
\text { large agglomerates of globules } \\
\text { on the surface }\end{array}$ & $\begin{array}{l}\text { Agglomerates of large seemingly } \\
\text { spherical calcite crystallites }\end{array}$ \\
\hline 2.0 & $\begin{array}{l}\text { Pancake-like crystallites with } \\
\text { small globules on the surface }\end{array}$ & $\begin{array}{l}\text { Aggregates of numerous nanosized } \\
\mathrm{CaCO}_{3} \text { crystallites }\end{array}$ \\
\hline 2.5 & Pancake-like crystallites & $\begin{array}{l}\text { Small laterally growing pancake-like } \\
\text { crystallites formed via association of } \\
\text { numerous nanosized } \mathrm{CaCO}_{3} \\
\text { crystallites along with other structures }\end{array}$ \\
\hline 3.0 & Pancake-like crystallites & $\begin{array}{l}\text { Structures formed by the association } \\
\text { of numerous nanosized } \mathrm{CaCO}_{3} \\
\text { crystallites }\end{array}$ \\
\hline 3.5 & Pancake-like crystallites & $\begin{array}{l}\text { Agglomerates of calcium carbonate } \\
\text { globules }\end{array}$ \\
\hline
\end{tabular}

Table 3.3: A summary of the different morphologies of calcium carbonate observed in SEM images of the chitosan-CaCO 3 composite obtained by mineralization via the McGrath method using $0.5 \mathrm{M} \mathrm{Ca}^{2+}$ and $\mathrm{HCO}_{3}^{-}(1 \mathrm{~h}$ alternate soaking +1 week soaking in Kitano solution) and varying concentrations of PAA w.r.t to dry weight of chitosan. 
(a)
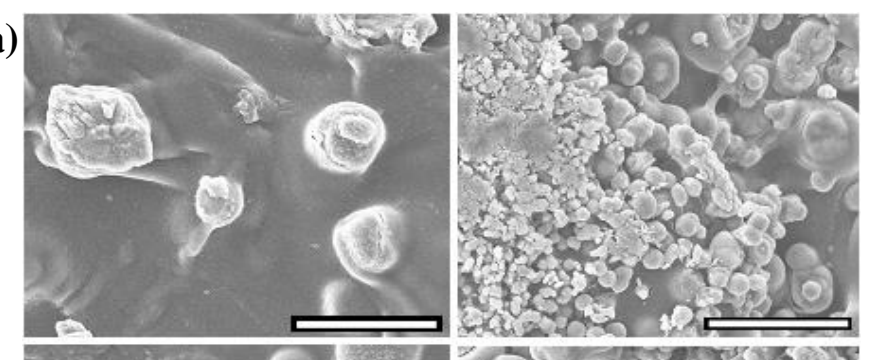

(b)

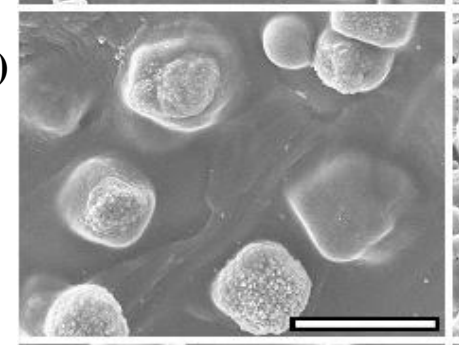

(c)

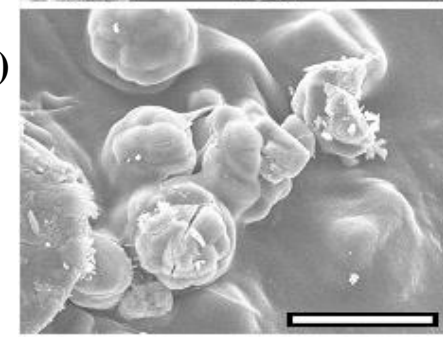

(d)

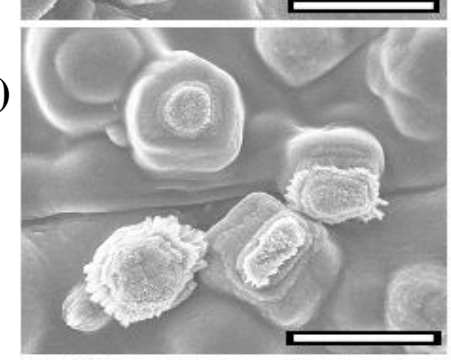

(e)

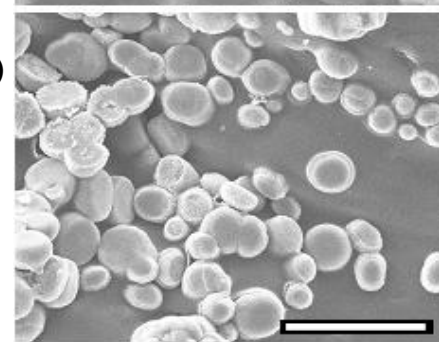

(f)

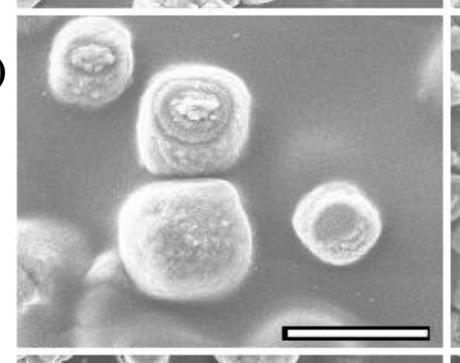

(g)

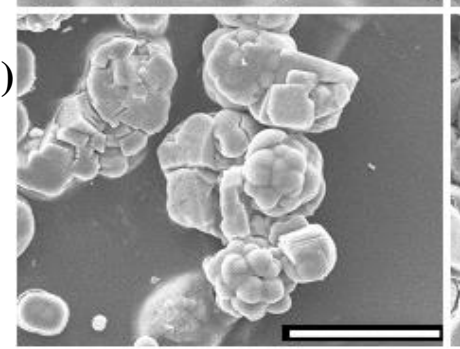

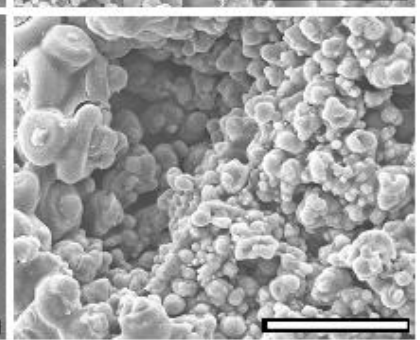
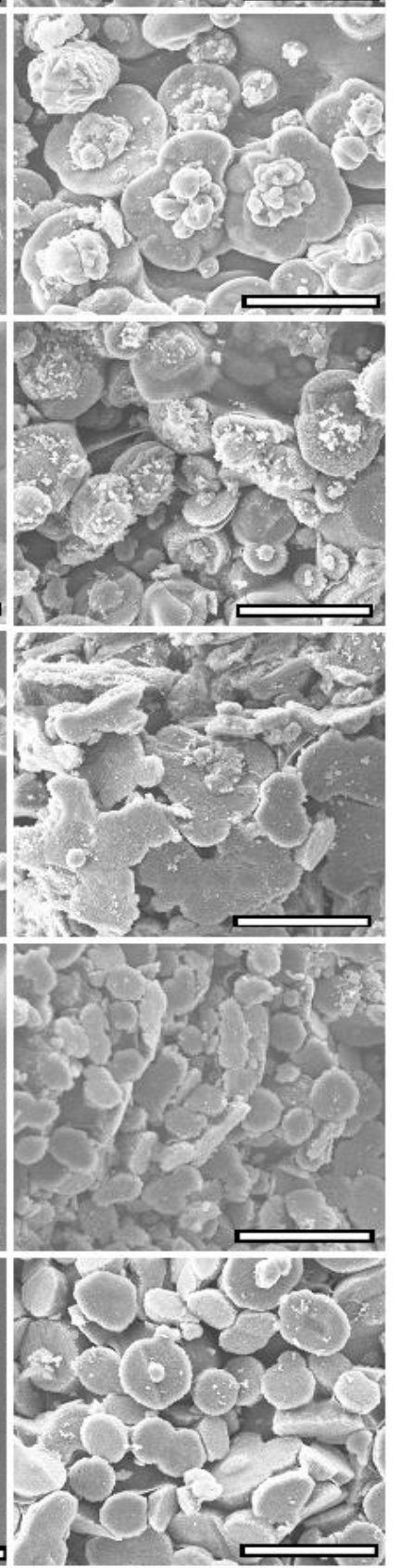

Figure 3.10: SEM images of morphology of $\mathrm{CaCO}_{3}$ crystallites found in between the printed layers (on the left-hand side) and on the regions exposed to the mineralization media (on the right-hand side) of $3 D$ printed scaffolds mineralized via the McGrath method using 0.5 M mineral precursor ion solutions (1 $h$ alternate soaking +1 week Kitano treatment) in the presence of (a) $0.5 \%$ (b) $1 \%$ (c) $1.5 \%$ (d) $2 \%$ (e) $2.5 \%(f) 3 \%(g) 3.5 \% w / w P A A$. Scale bar is $50 \mu \mathrm{m}$. 
In between the layers of the scaffold, the crystallite growth was not as straightforward. Here, with the increase in PAA concentration, its influence on the aggregation of the nanocrystallites to form larger structures and the lateral growth of the crystallites is evident. Studies suggest that the morphology of the resulting $\mathrm{CaCO}_{3}$ crystallites nucleating on a substrate is an interplay between the concentration of PAA and $\mathrm{Ca}^{2+}$ ions in the mineralization solution and the orientation of the substrate on which the nucleation takes place [39]. It is also suggested that although lateral growth of $\mathrm{CaCO}_{3}$ crystallites is typical in the presence of PAA, it is the orientation of the PAA-Ca ${ }^{2+}$ complex (part of the polyelectrolyte complex) formed on the substrate during the initial stages of crystal growth that is a determinant of the morphology of the final $\mathrm{CaCO}_{3}$ crystallites formed. This is again dependent on the concentration of PAA and the mineralization solution. In the regions between the printed layers, there is an obvious reduction in the concentration of the precursor ions and PAA compared with that present in the regions of the scaffold directly exposed to mineralization media. This along with the fact that the crystal growth is also occurring in the presence of overlying hydrogel layers could be the reason for the variation in the morphology of the crystallites from layer to layer.

\subsubsection{Precursor ions concentration}

An appropriate influx of precursor ions is important for the formation and distribution of pancake-like crystallites throughout the scaffold. Based on the data discussed above, the crystal growth modifier/additive concentration was kept constant at $2.5 \%$ w/w PAA w.r.t the dry weight of chitosan used and the effects of precursor ion concentration on the extent of mineralization and the morphology of $\mathrm{CaCO}_{3}$ formed in the 3D chitosan hydrogel-based scaffold was explored. For this study, the scaffolds were mineralized via the McGrath method in the presence of $2.5 \%$ w/w PAA (w.r.t dry wt. of chitosan) wherein, the scaffolds were exposed to mineral precursor ion solutions alternately for $24 \mathrm{~h}$ each and further soaked in Kitano solution for 1 week. The concentration of the precursor mineral ion solutions was varied from $0.1 \mathrm{M}$ to $0.5 \mathrm{M}$.

Similar to the observations described above, variations in the crystallite morphology were observed as the concentration of the precursor ion was increased. The region in between the layers of the 3D scaffold was found to be more sensitive to variations in the mineralization process. Hence, the data presented here are for the growth occurring in between the layers of the scaffold only. 
Across all precursor ion concentrations, the effect of PAA as the crystal growth modifier is evident in terms of the growth of $\mathrm{CaCO}_{3}$ crystallite by aggregation of nanosized crystallites in association with the hydrogel matrix resulting from the formation of polyelectrolyte complexes between the hydrogel matrix and the PAA. However, the resultant morphology of the crystallites was not solely flat pancake-like as is evident in Figure 3.11. Nonetheless, when using precursor ion solutions of low concentration; $0.1 \mathrm{M}$, the size of the resultant nanoparticle aggregates is evidently smaller and there is a preference for pancake-like growth, although the extent of mineralization is low. When using precursor ion solutions of concentration $0.25 \mathrm{M}$, results in crystallites that are mostly micron-sized laterally grown aggregates of nanosized crystallites similar to the other cases. When using $0.5 \mathrm{M}$ precursor ion solutions, the resultant crystallites are in the form of larger micron-sized aggregates $(30.2 \pm 4.7 \mu \mathrm{m})$ of nanosized $\mathrm{CaCO}_{3}$ crystallites.
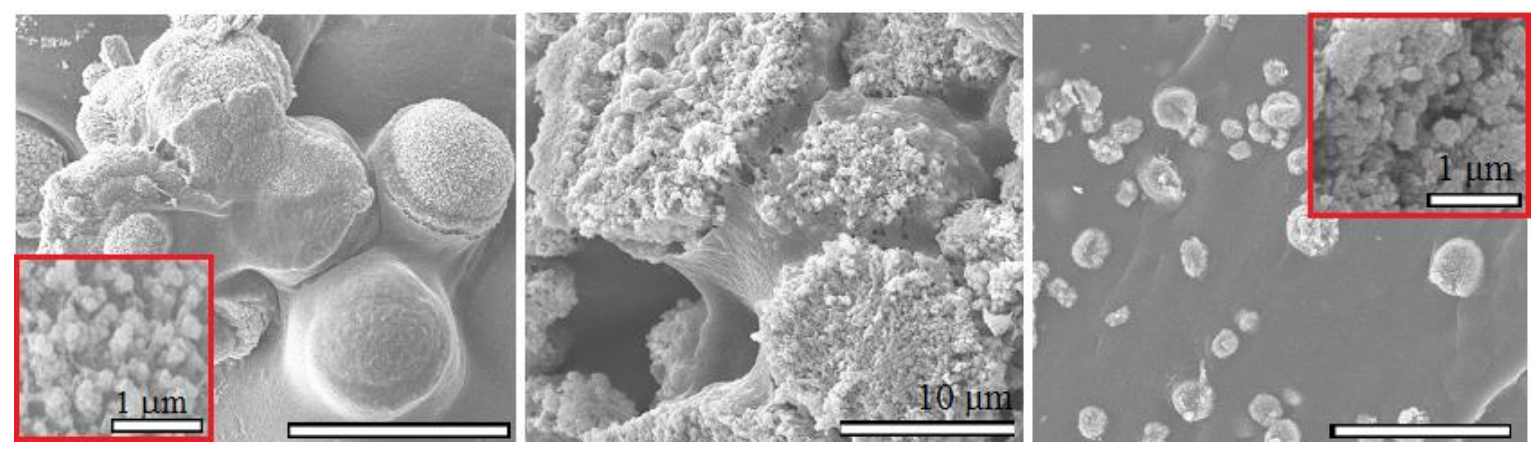

Figure 3.11: SEM images of $\mathrm{CaCO}_{3}$ crystallites formed in between the layers of the $3 \mathrm{D}$ scaffolds mineralized via the McGrath mineralization method (24 halternative soaking +7 days Kitano) in the presence of 2.5\% PAA using mineral precursor ion solutions of concentrations (a) $0.5 \mathrm{M}$; (b) $0.25 \mathrm{M}$; and (c) $0.1 \mathrm{M}$. Insets are higher magnification images of the crystallites showing the aggregation of nano-sized crystallites. Scale bar is $50 \mu \mathrm{m}$ unless otherwise stated.

In order to further optimize the crystallite morphology, the period of exposure to the mineralization media was also investigated. Along with the solution concentration, this factor also affects crystal growth kinetics.

\subsubsection{Period of exposure to mineralization media}

In order to investigate the effect of the period of exposure of the scaffolds to the mineralization media on the resultant crystallite morphology the following experiments were conducted: 
a) scaffolds were mineralized by alternately soaking in mineral precursor ion solution $\left(\mathrm{Ca}^{2+}\right.$ and $\mathrm{CO}_{3}{ }^{2-}$ ion solutions) only (one alternate cycle) to study the growth of seed $\mathrm{CaCO}_{3}$ crystals. Here the soaking period in each of the solutions was varied from 1 - $24 \mathrm{~h}$; and

b) scaffolds were mineralized via the McGrath mineralization method in which the period of exposure to the Kitano solution was varied from 3 days to 7 days by keeping the alternate soaking period constant based on the observations from (a).

All of the above mineralization experiments were performed in the presence of $2.5 \% \mathrm{w} / \mathrm{w}$ PAA w.r.t to the dry weight chitosan used. Two different series were used to study the effect of mineralization, one where only alternate soaking in precursor ion solutions was applied and the second where both alternate soaking + Kitano solutions were used i.e., the McGrath method. It can however be assumed that under similar mineralization conditions, the crystal growth kinetics and the resultant morphology of the crystallites formed during the alternate soaking step would always be similar, and that therefore the observations from the SEM images obtained after air-drying such scaffolds can be used to infer what is initially occurring when the McGrath method is used and therefore for interpretation of the morphology data.

\subsection{5a 0.5 M precursor ion solution}

When 3D chitosan hydrogel-based scaffolds were mineralized by alternately soaking in 0.5 $\mathrm{M}$ precursor ion solutions only, both the morphology of the resultant $\mathrm{CaCO}_{3}$ crystallites formed in between the layers of the scaffold and the extent of mineralization that occurred varied with the time period for which the scaffolds were soaked in mineralization media being increased from $1 \mathrm{~h}$ to $24 \mathrm{~h}$. The size of the crystallites increased with the exposure time. SEM images showed that the resultant crystallites formed in between the layers are predominantly globular and that such structures were prominent within $1 \mathrm{~h}$ of alternate exposure to the precursor ion solutions (Figure 3.12; top row). At this high concentration of the mineral precursor ions, extensive mineralization is obtained throughout the scaffold.

Furthermore, when the 3D scaffolds were mineralized via the McGrath method in the presence of $0.5 \mathrm{M}$ precursor ion solution (1 h each alternate soaking + soaking in Kitano solution for 1 week), very extensive mineralization in the form of globular crystallites was obtained on the exposed regions of the scaffolds with a few pancake-like structures formed in between the layers. Also, when using the McGrath method, as the exposure time to the 
precursor mineral ion solutions is increased (from $1-24 \mathrm{~h}$ ), keeping the exposure to Kitano solution constant at one week, the crystallite morphology throughout (exposed and in between the layers) the scaffold was predominantly globular aggregates of nano-sized crystallite-like structures (similar to those shown in Figure 3.11 (a))
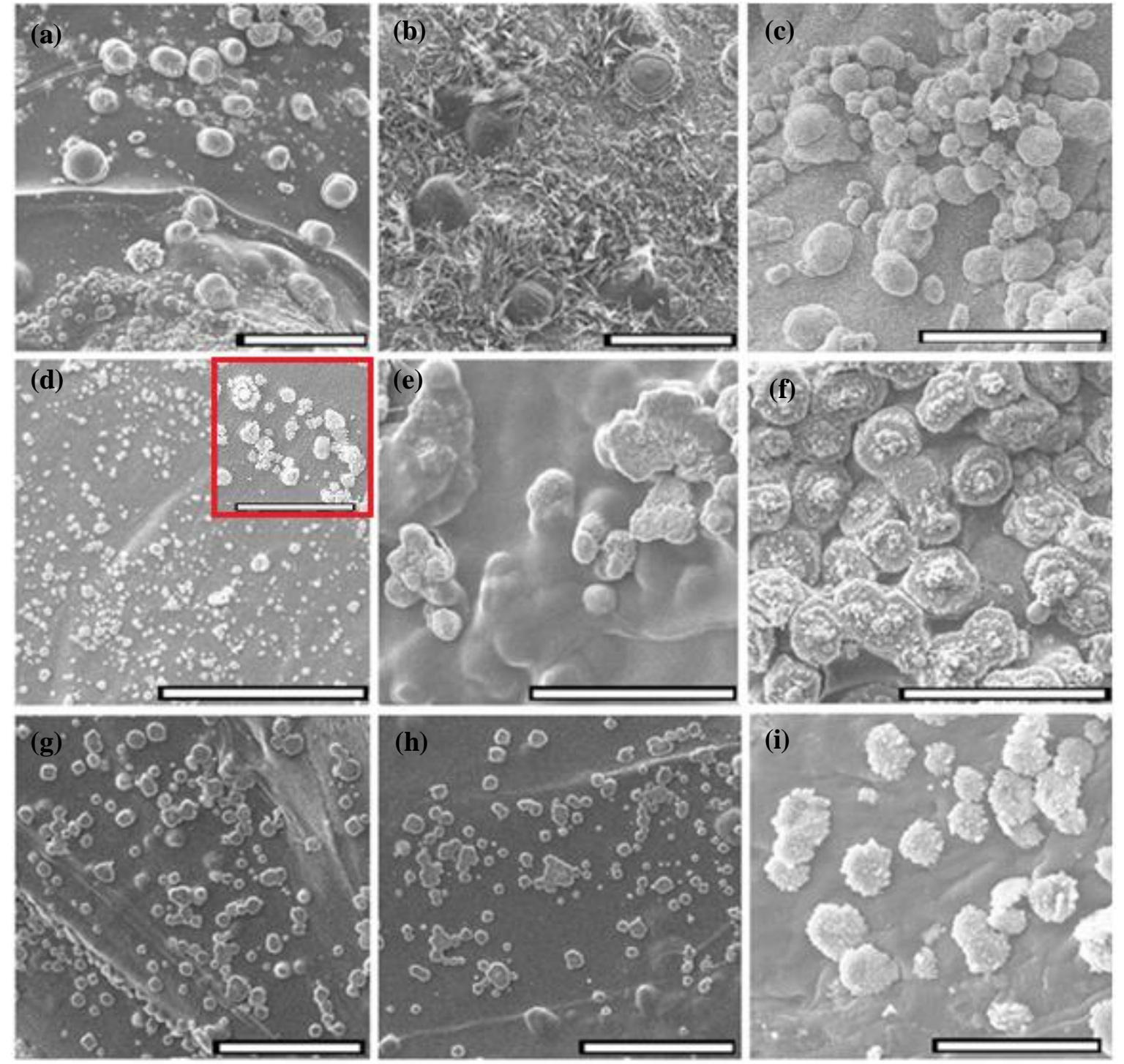

Figure 3.12: SEM images of calcium carbonate crystallites formed in between the layers of the $3 D$ chitosan hydrogel-based scaffold as a result of alternately soaking in $0.5 \mathrm{M}$ mineral precursor ion (top row) solution for (a) $1 \mathrm{~h}$, (b) $2 \mathrm{~h}$ and (c) $24 \mathrm{~h}$; $0.25 \mathrm{M}$ mineral precursor ion solution (middle row) for (d) 1 h (inset is the higher magnification image of the same), (e) $2 \mathrm{~h}$ and (f) $24 \mathrm{~h}$; and $0.1 \mathrm{M}$ mineral precursor ion solution (bottom row) for $(\mathrm{g}) 1 \mathrm{~h}$, $(\mathrm{h}$ ) $2 \mathrm{~h}$ and (i) $24 \mathrm{~h}$. Scale bar of the images is $50 \mu \mathrm{m}$ and that for inset image is $10 \mu \mathrm{m}$. 


\subsection{5b 0.25 M precursor ion solution}

When 3D chitosan hydrogel-based scaffolds mineralized by alternately soaking in $0.25 \mathrm{M}$ precursor ion solutions only, small (average $1.43 \pm 0.27 \mu \mathrm{m}$ in diameter) laterally growing aggregates of $\mathrm{CaCO}_{3}$ crystallites were observed in between the layers of the scaffold (Figure 3.12; middle row) after $1 \mathrm{~h}$ exposure to the precursor ion solutions. The crystallites were smaller, and the extent of mineralization was lower compared to that of the scaffolds mineralized via alternate soaking in $0.5 \mathrm{M}$ mineral precursor ion solutions for the same exposure period. The size of the seed crystallites in the form of aggregates of nanosized crystallites within the hydrogel matrix increased with the exposure time. After $24 \mathrm{~h}$ exposure, the resultant crystallites were mostly pancake-like with an average size of $15.9 \pm$ $2.6 \mu \mathrm{m}$ in diameter, however, random structures were also seen to grow on the surface of the pancake-like crystallites.

Based on the observed crystal growth pathway as a function of the soaking time in the case of $3 \mathrm{D}$ scaffolds alternately soaked in $0.25 \mathrm{M}$ mineral precursor ion solution only, it is apparent that smaller seed crystals such as the ones obtained within the first hour of exposure to the precursor ions could lead to the formation of larger pancake-like structures with prolonged exposure to mineralization solutions such as saturated $\mathrm{CaCO}_{3}$ solution (Kitano). This is ascertained in the SEM images shown in Figure $3.13(a, b)$ depicting the crystallite morphology after exposure of pre-soaked chitosan hydrogel-based scaffolds (alternate soaking in mineral precursor ion solutions for $1 \mathrm{~h}$ each) to Kitano solution for 3 and 4 days respectively. Figure 3.13 (a) shows that the resultant morphology after 3 days exposure to Kitano solution is very close to the preferred pancake-like structure.

Increasing the precursor ion concentration resulted in considerable mineralization throughout the scaffold. However, the larger globular crystallites with an average diameter of $14.6 \pm 2.2 \mu \mathrm{m}$ formed after $1 \mathrm{~h}$ alternate soaking in $0.5 \mathrm{M}$ precursor ion solutions did not result in pancake-like crystallites when the McGrath mineralization method was used. This mineralization protocol resulted rather in the formation of micron-sized globular aggregates of nanosized crystallites. There is evidently, a trade-off between the extent of mineralization and the morphology of crystallites that are obtained. 



Figure 3.13: SEM images of the morphology of $\mathrm{CaCO}_{3}$ crystallites formed in between the layers of the 3D chitosan hydrogel-based scaffolds mineralized via the McGrath method by exposure to (a) $0.25 \mathrm{M}$ precursor ion solution alternately for 1 h each +3 days Kitano treatment; (b) $0.25 \mathrm{M}$ precursor ion solution alternately for $1 \mathrm{~h}$ each +4 days Kitano treatment; (c) only Kitano solution for 7 days; (d) 0.1 M precursor ion solution alternately for 2 h each + 3 days Kitano treatment; (e) 0.1 M precursor ion solution alternately for $2 \mathrm{~h}$ each +4 days Kitano treatment (inset is a higher magnification image of the same) and $(f)$ 0.1 M precursor ion solution alternately for 2 h each +7 days Kitano treatment. Scale bar of the images is $50 \mu \mathrm{m}$.

\subsection{5c $0.1 \mathrm{M}$ precursor ion solution}

When 3D chitosan hydrogel-based scaffolds were mineralized by alternately soaking in 0.1 $\mathrm{M}$ precursor ion solutions only, the extent of mineralization was the least compared to the others, nonetheless, the resultant crystallites are mostly laterally growing pancake-like structures (Figure 3.12, bottom row). Although pancake-like crystallites (average $5.96 \pm$ $0.74 \mu \mathrm{m}$ diameter) were observed within $1 \mathrm{~h}$ exposure to the mineral precursor ion solutions, the extent of mineralization in between the layers of the scaffold was very low. Increasing the exposure time to $2 \mathrm{~h}$, resulted in the formation of similar sized pancake-like seed crystallites (average $4.7 \pm 1.08 \mu \mathrm{m}$ diameter) but the extent of mineralization throughout the layers of the scaffold improved. (Note that the images in Figure $3.12(\mathrm{~g}, \mathrm{~h})$ are 
representative of the morphology of the crystallites present in between the scaffold layers and they are taken from the regions with the greatest number of crystallites present, and as such these images are not representative of the extent of mineralization throughout the scaffold and therefore the homogeneity of the crystallization).

In the case of 3D chitosan hydrogel-based scaffolds mineralized via the McGrath method (alternatively soaking $0.1 \mathrm{M}$ precursor ion solutions (exposure time varying from $1 \mathrm{~h}$ to 24 h) followed by soaking in Kitano solution (1 week)), the extent of mineralization throughout the scaffold was increased in comparison to when mineralization was initiated via only alternate soaking. Figure 3.13 (d-f) shows the evolution in the morphology of the resultant crystallites after exposure of the pre-soaked chitosan hydrogel-based scaffolds ( $2 \mathrm{~h}$ alternate soaking in mineral precursor ion solution) to Kitano solution for 3, 4 or 7 days. Although the extent of mineralization is very low in comparison to that of scaffolds mineralized under similar conditions using $0.25 \mathrm{M}$ or $0.5 \mathrm{M}$ precursor ion solutions, the morphology of the resultant crystallites was mostly aggregates of nano-sized crystallites. Along with these aggregate-like structures, structures similar to those shown in Figure 3.13 (e) (2 h alternate soaking +4 days Kitano treatment) which are very similar to the desired pancake-like morphology were also observed.

Exposure of 3D chitosan hydrogel-based scaffolds to only Kitano solution for an extended period of time ( 1 week) in the presence of $2.5 \%$ PAA (w.r.t dry weight of chitosan used) can also lead to the formation of only pancake-like crystallites throughout the scaffold (Figure 3.13 (c)). However, considering that the extent of mineralization in between the layers of the scaffold is the least of all the mineralization methods, with respect to the aim of this thesis; to be able to straightforwardly manipulate the system to achieve a range of different extents of mineralization throughout the 3D scaffold, this is not the most preferred mineralization method.

Based on these results, it can be suggested that the concentration of the mineralization solutions and the period of exposure of the scaffolds to the mineralization media during the alternate soaking step is a determinant step with regard to the morphology of the final crystallites formed. The preferred morphology of seed crystals formed after alternate soaking in the mineral precursor ion solutions is aggregates of nano-sized crystallites in the form of small pancake-like structures. These seed crystals would then preferably grow in size with further extended soaking in Kitano solution. This is supported by the observation 
that smaller pancake-like seed crystallites of about $4.7 \pm 1.08 \mu \mathrm{m}$ obtained by alternately soaking in $0.1 \mathrm{M}$ mineral precursor ion solutions for $2 \mathrm{~h}$ each resulted in the preferred pancake-like crystallites with further Kitano treatment (4 days) (Figure 3.13 (e)).

\subsubsection{Extent of mineralization}

The extent of mineralization throughout the 3D chitosan hydrogel-based scaffold is governed by a diffusion driven process involving the transportation of ions to different regions of the scaffolds. The rate of diffusion is increased with the concentration and $\mathrm{pH}$ of the mineralization solution. Results of all the above mineralization studies suggest that the McGrath mineralization method increases the extent of mineralization in comparison to Kitano mineralization alone (Figure 3.13 (c)), where the mineralization solution is near neutral $\mathrm{pH}$ and the mineralization process is dependent on the change in $\mathrm{pH}$ by slow evolution of $\mathrm{CO}_{2}$. Whereas in the case of the McGrath method, the initial alternate soaking step wherein the mineral precursor ion solutions are at a slightly acidic $\mathrm{pH}$, causes deprotonation of some of the chitosan hydrogel chains, facilitating a $\mathrm{pH}$-dependent diffusion of the mineral ions throughout the scaffold. At a precursor ion concentration of $0.5 \mathrm{M}$, good mineralization is observed on the regions of the scaffold exposed to the mineralization media as well as in between the layers of the scaffold. The extent of mineralization in between the layers decreased as the concentration of the precursor ions was decreased to $0.25 \mathrm{M}$ and further lowered in the case of $0.1 \mathrm{M}$.

Another factor that may contribute to increasing the extent of mineralization in between the scaffold layers is the presence of seed crystals formed during the alternating step when using the McGrath mineralization method. These seed crystals act as the low energy sites that attract and accelerate the rate of nucleation and growth in the subsequent Kitano step that follows. When 3D scaffolds are mineralized via soaking in Kitano solution only, the scaffold merely plays the role of a heterogeneous nucleation site for the calcium carbonate molecules in Kitano solution and so the minerals ions may not penetrate as deep into the layers of the scaffold as in the case when the McGrath method is used. Hence, leading to lower mineralization in between the layers compared to the regions directly exposed to the mineralization solution. When Kitano is assisted by the alternate soaking step (as in the McGrath method), seed crystals are formed throughout the 3D matrix of the hydrogel scaffold, which promotes extensive nucleation and growth of crystallites across the entire volume of the scaffold. XRD analysis (Figure 3.14) of 3D chitosan hydrogel-based scaffolds 
mineralized via different techniques in the presence of $2.5 \%$ w/w PAA (w.r.t to dry wt. of chitosan) is also in agreement with these observations.

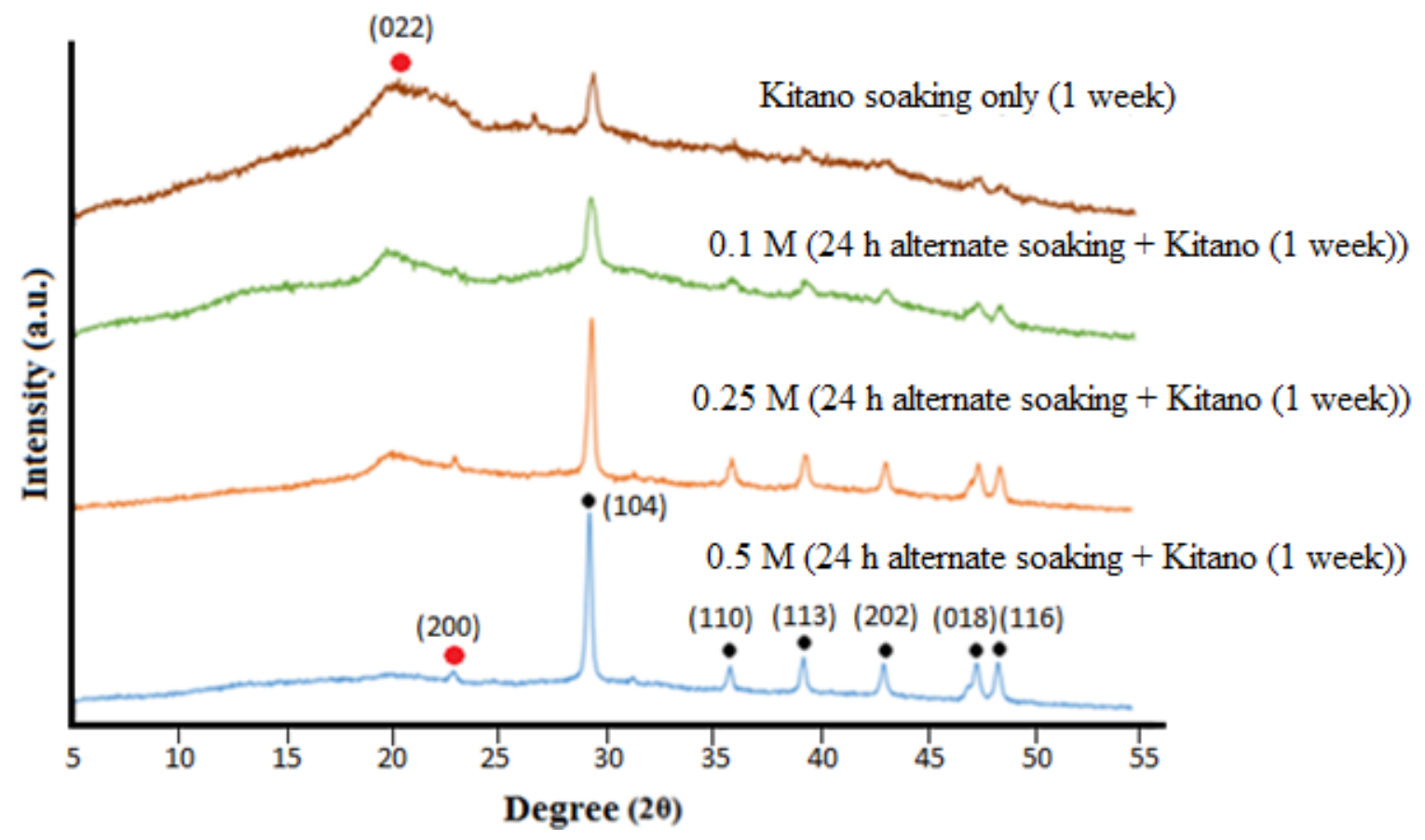

Figure 3.14: XRD plots for 3D chitosan hydrogel-based scaffolds mineralized via the McGrath method or only soaking in Kitano solution in the presence of PAA (2.5\% w/w w.r.t to dry wt. of chitosan). The black and red dots represent the prominent $2 \theta$ peaks corresponding to calcite and crystalline planes in chitosan respectively.

This data (Figure 3.14) confirm that the extent of mineralization decreases with a decrease in the concentration of the mineral precursor ions, as was indicated from the SEM analysis. This is most readily evidenced by the appearance of a prominent broad $2 \theta$ peak at $19.3^{\circ}$ corresponding to (022) plane of chitosan and the disappearance of the prominent peak at $29.3^{\circ}$ corresponding to (104) plane of the calcite polymorph of $\mathrm{CaCO}_{3}$ as the concentration of precursor ions was reduced from $0.5 \mathrm{M}$ to $0.1 \mathrm{M}$ [40]. The broadest chitosan peak was observed in the case of scaffolds mineralized by soaking only in Kitano solution for one week. Figure 3.13 (c) shows that the extent of mineralization in between the printed layers was low when chitosan hydrogel-based scaffolds were mineralized via the Kitano method in the presence of $2.5 \%$ PAA. This observation is supported by the observed broad peak corresponding to the (022) plane of chitosan. The opposite is observed in the case of the scaffolds mineralized via the McGrath method in the presence of 2.5\% PAA using $0.5 \mathrm{M}$ precursor ion solution ( $24 \mathrm{~h}$ alternate soaking +1 week Kitano treatment) wherein a sharp 
calcite peak observed at $29.3^{\circ}$ and the chitosan peak has practically vanished. This reveals extensive mineralization throughout the printed layers and the exterior surface of the scaffold. In the case of 2D chitosan hydrogel films mineralized via the McGrath method in the presence of PAA, it was observed that a small percentage of vaterite was obtained, however, in the case of 3D chitosan hydrogel-based scaffolds, for all mineralization methods used calcite was the only polymorph formed [41].

A quantitative analysis of the mineral content within 3D chitosan hydrogel-based scaffold was performed using TGA. Figure 3.15 is a representative weight loss (in percentage) versus temperature plot from the same. The percentage mineral content is expressed in terms of the percentage residual volume remaining after the thermal decomposition of the composite over a selected range of temperatures. For this study, 3D chitosan hydrogel-based scaffolds were mineralized via the McGrath method by alternately soaking in $0.1 \mathrm{M}-0.5 \mathrm{M}$ precursor ion solutions for $24 \mathrm{~h}$ followed by soaking in Kitano solution for one week. This mineralization protocol was used for this study as representative composite scaffolds with the highest mineral content obtained when mineralization is performed in the presence of $0.5 \mathrm{M}, 0.25 \mathrm{M}$ or $0.1 \mathrm{M}$ precursor ion solutions. For comparison, chitosan hydrogel-based scaffolds were also mineralized by soaking in Kitano solution for 1 week only, as a representative composite with the least mineralization achieved in this study.

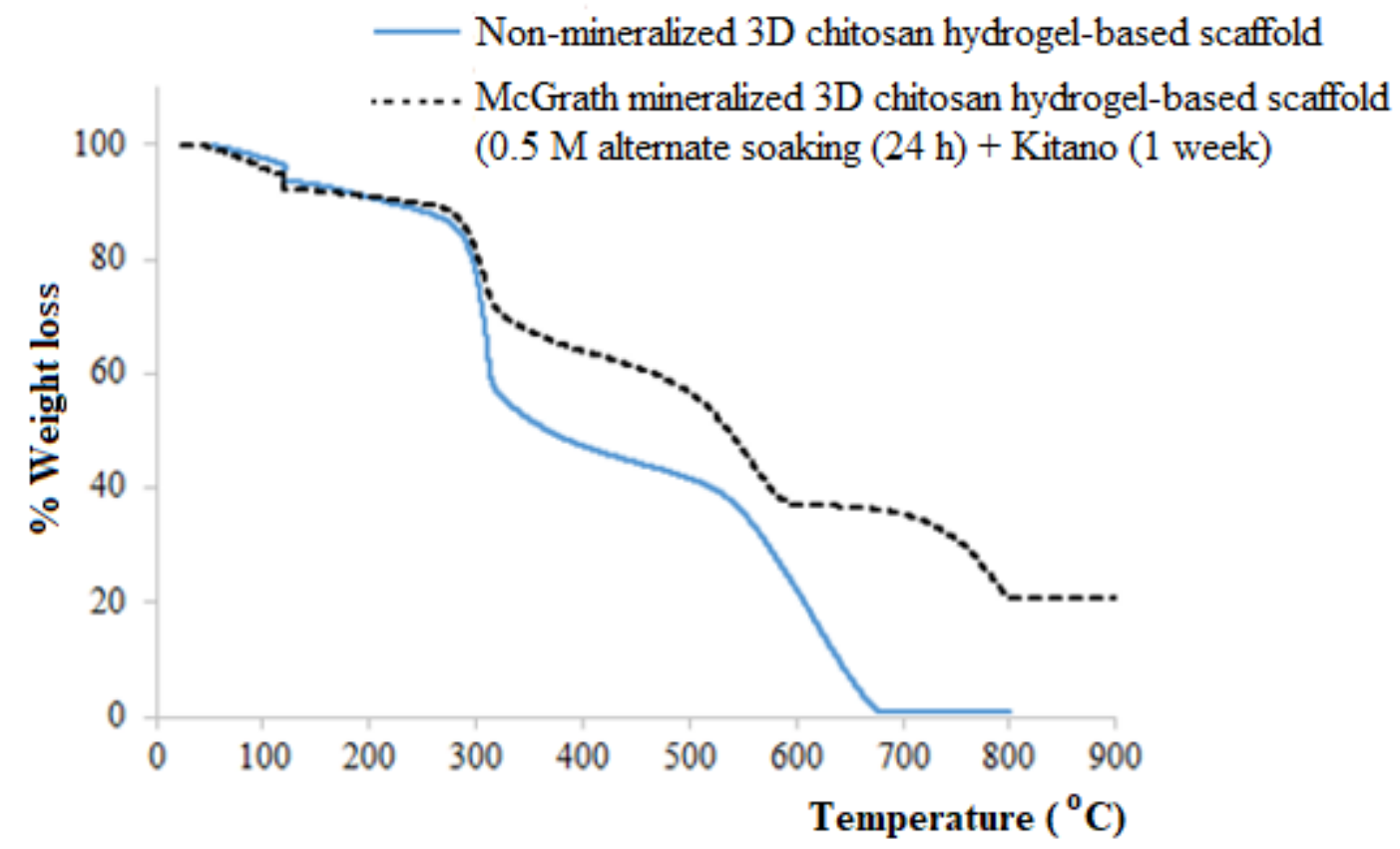

Figure 3.15: A representative TGA plots for McGrath mineralized and non-mineralized chitosan hydrogel-based scaffolds. 
During TGA analysis, the pure chitosan scaffolds undergo a three-step decomposition from $0{ }^{\circ} \mathrm{C}-900^{\circ} \mathrm{C}$. The initial weight loss corresponds to the loss of moisture at about $100{ }^{\circ} \mathrm{C}$, the next step corresponds to the decomposition of chitosan at about $310^{\circ} \mathrm{C}$, the final weight loss step beyond $520^{\circ} \mathrm{C}$ corresponds to oxidation of partially decomposed chitosan and further charring beyond $680^{\circ} \mathrm{C}$. In the case of mineralized scaffolds, decomposition beyond $680{ }^{\circ} \mathrm{C}$ leads to a fourth-step corresponding to the final decomposition of the inorganic components; $\mathrm{CaCO}_{3}$ to $\mathrm{CaO}$ [15]. Figure 3.15 shows a typical TGA curve for McGrath mineralized composites and non-mineralized chitosan hydrogel-based scaffolds. Ideally, the total residual volume beyond $600{ }^{\circ} \mathrm{C}$ should correspond to the total calcium carbonate content within the tested samples, since based on literature decomposition of calcium carbonate to $\mathrm{CaO}$ occurs between $600{ }^{\circ} \mathrm{C}-800{ }^{\circ} \mathrm{C}$ [42]. Theoretically, therefore the residual volume at the beginning of the $4^{\text {th }}$ degradation step $\left(\sim 580{ }^{\circ} \mathrm{C}\right)$ should represent the total $\mathrm{CaCO}_{3}$ content within the sample.

Chemical stoichiometry can be used to ascertain the values obtained from TGA analysis by comparison with the total amount of calcium carbonate formed as a result of the reaction between calcium chloride and sodium hydrogen carbonate. However, in the case of the mineralization method used in this research, this method is not appropriate considering that $\mathrm{CaCO}_{3}$ nucleation occurs not only in the chitosan hydrogel matrix but also on any other surface the mineralization media is in contact with. Thus, another experiment was performed to allow the estimation of the total calcium carbonate content to the TGA results. The air-dried mineralized samples were crushed and reacted with $10 \mathrm{~mL}$ of $0.5 \mathrm{mM} \mathrm{HCl}$ ( $\mathrm{pH} 4.3$ ) (replenished every $24 \mathrm{~h}$ ); $\mathrm{CaCO}_{3}$ reacts with $\mathrm{HCl}$ to form water-soluble $\mathrm{CaCl}_{2}$; the unreacted chitosan is then filtered and weighed after air-drying. The solution was changed every day over a period of 4 days.

Table 3.4 gives the mineral content $(\%)$ within chitosan hydrogel-based scaffolds mineralized via the McGrath method estimated via the two techniques: TGA and the dissolution analysis. Considerable differences are evident in the values obtained. This is because both techniques have some analytical disadvantages when used to analyse chitosancalcium carbonate composites. 


\begin{tabular}{|c|c|c|}
\hline \multirow{2}{*}{ Mineralization Technique Used } & TGA & Acid dissolution \\
\cline { 2 - 3 } & (lower limit) \\
\cline { 2 - 3 } & (upper limit) & $11.6 \pm 3.1$ \\
\hline $\begin{array}{c}\text { Alternate soaking in 0.5 M precursor } \\
\text { ion solutions (24 h) + Kitano (1 week) }\end{array}$ & $36.8 \pm 2.1$ & $10.0 \pm 1.3$ \\
\hline $\begin{array}{c}\text { Alternate soaking in 0.25 M precursor } \\
\text { ion solutions (24 h) + Kitano (1 week) }\end{array}$ & $20.2 \pm 0.9$ & $4.4 \pm 0.2$ \\
\hline $\begin{array}{c}\text { Alternate soaking in 0.1 M precursor } \\
\text { ion solutions (24 h) + Kitano (1 week) }\end{array}$ & $14.9 \pm 1.6$ & $2.0 \pm 1.2$ \\
\hline $\begin{array}{c}\text { Soaking in Kitano solution (saturated } \\
\text { CaCO } 3 \text { solution) (1 week) }\end{array}$ & $10.2 \pm 1.5$ & \\
\hline
\end{tabular}

Table 3.4: The percentage calcium carbonate content within 3D chitosan hydrogel-based scaffolds mineralized via the McGrath method using increasing concentrations of mineral precursor ions or Kitano method in the presence of $2.5 \% \mathrm{w} / \mathrm{w}$ PAA w.r.t dry weight of chitosan used, as analysed via TGA and acid dissolution method. For TGA, the percentage weight loss for one sample is obtained by averaging the \% weight loss obtained for small portions ( 25 mg) of the complete composite sample until the whole scaffold was thermally analysed. Three replicates of each type were analysed via both techniques and averaged to obtain the stated values.

In the case of TGA analysis, incomplete decomposition of the organic matrix occurs due to the intrinsic association between the mineral and the polymer matrix resulting in higher estimated percentage residual volume. Despite this, the percentage residual weight at the beginning of the $4^{\text {th }}$ step (approximately $580^{\circ} \mathrm{C}$ ) provides a good approximation of the total mineral content of the sample since ideally decomposition of inorganic calcium carbonate occurs between $600{ }^{\circ} \mathrm{C}-800{ }^{\circ} \mathrm{C}$. Thus, this value is assumed to be the upper limit of the total mineral content of the composites fabricated using the McGrath method. (Noting that under this assumption the percentage of undecomposed chitosan is comparably very low.) Similarly, in the case of the acid dissolution experiment, the ideal pH (1 - 3) for maximum dissolution of calcium carbonate falls within the solubility range for chitosan $(<5.2)$. This means that some dissolution of chitosan will occur if the concentration of the acid is increased in an effort to achieve maximum calcium carbonate dissolution, thus reducing the 
sensitivity of the experiment. Hence, the concentration of $\mathrm{HCl}$ used was kept low such that minimal dissolution of chitosan might occur and the solution was replenished occasionally to increase the dissolution rate of calcium carbonate. Due to both chitosan and calcium carbonate dissolving at the $\mathrm{pH}$ values, the estimated percentage of calcium carbonate content within the composite is here considered as the lower limit of the actual total mineral composition. Given that TGA is the more straightforward of the two methods and that relatively the amount of chitosan remaining bound to or occluded in the calcium carbonate will be small compared with the observed mutual dissolution occurring in the chemical method, it was the method used to obtain an appropriate upper estimate to the actual mineral content of all samples.

Both sets of data ideally indicate that relative proportions of organic to inorganic in the final composite materials can be readily manipulated by changing the concentration of the mineralization solutions and exposure times. At least a two-fold range in the mineral content can be achieved via the McGrath method.

A critical observation from the acid dissolution experiments was that even when the acid concentration was as high as $0.05 \mathrm{M}(\mathrm{pH} 1.3)$, some amount of calcium carbonate remained (visible as white patches under physical observation) after $2 \mathrm{~h}$ of dissolution. This may suggest that the interaction between the mineral and organic matrix is rather strong.

The XRD data shown in Figure 3.14, indicating the variation in the extent of mineralization with mineral precursor ion solution concentrations, is supported by the TGA and acid dissolution data summarised in Table 3.4 showing that as the concentration of the precursor mineral ion solution that the scaffolds are exposed to, is increased, the percentage mineral content (percentage residual volume) of the resultant composite is also subsequently increased.

\subsubsection{Effect of printer-induced macropores on the extent of mineralization}

Another factor that may affect the extent of mineralization within a 3D hydrogel-based scaffold is the presence of the printer-induced macropores. To study this effect firstly, scaffolds were fabricated via two different techniques: bulk gelation; and 3D printing. In both cases, the scaffolds were then mineralized via the McGrath method ( $24 \mathrm{~h}$ alternate soaking +1 week Kitano treatment $)$ in the presence of PAA $(2.5 \%$ w/w w.r.t to dry wt. of chitosan) using $0.5 \mathrm{M}$ precursor ion solution. To fabricate scaffolds via bulk gelation, a 15 
$\mathrm{mm}$ diameter $\times 16 \mathrm{~mm}$ high cylindrical column of chitosan hydrogel was deprotonated over a week in the presence of $1 \mathrm{M} \mathrm{NaOH}$, rendering it insoluble and hence assuming the shape a solid cylindrical 3D scaffold without any macro-sized pores (optical image of the scaffold shown in Figure 3.17). 3D printed scaffolds (15 mm diameter $\times 16 \mathrm{~mm}$ high) on the other hand were fabricated with perpendicularly aligned cubic lattices with either $1.5 \mathrm{~mm} \times 1.5$ mm macropores.
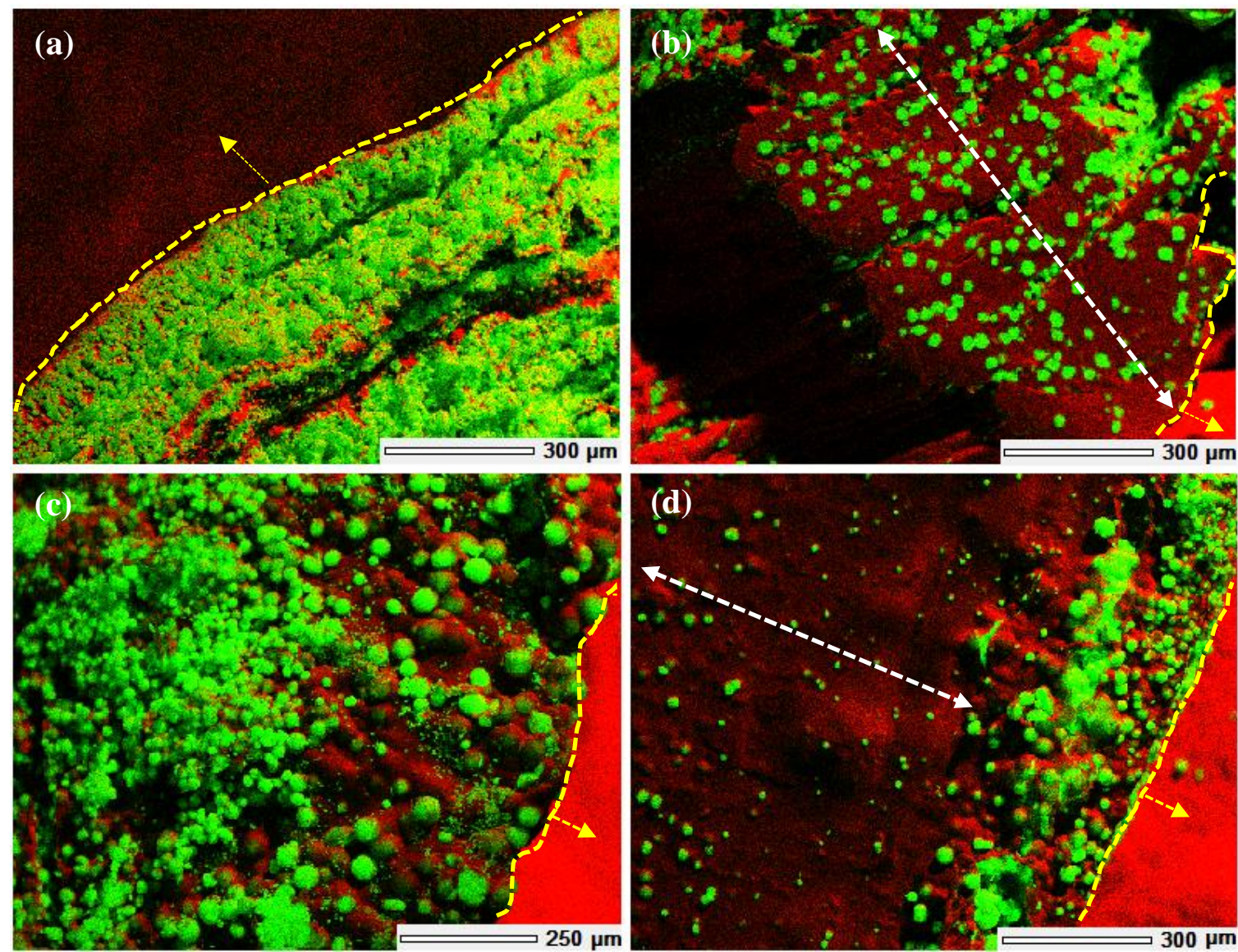

Figure 3.16: EDS image of chitosan hydrogel-based composites fabricated via $(a, b) 3 D$ printing and $(c, d)$ bulk gelation and mineralized via the McGrath mineralization method in the presence of $2.5 \%$ PAA; $(a, c)$ show the external surface of the scaffold directly in contact with the mineralization solution and $(b, d)$ show the transverse cross-section of the samples (region not exposed directly to the mineralization solution). Calcium is shown in green and carbon in red. The region marked by the white arrows represent those not exposed to the mineralization solution directly. The regions marked by yellow dashes and arrows represent the external surfaces that are not a part of the sample.

The EDS images of the cross-sections of composites fabricated via 3D printing with printed macropores and those developed via bulk gelation without any macropores are shown in 
Figure 3.16. This data suggest that high mineralization occurred in both of the cases on the exterior surfaces of the scaffolds which are in direct contact with the mineralization media. However, the depth of mineralization towards the interiors of the scaffold was higher in the case of 3D printed scaffolds containing macropores compared to when no macropores are present. The introduction of macropores as well as the layer-by-layer build of the scaffolds increases the area available for diffusion of the mineral ions into the scaffold and hence increases the extent of mineralization occurring throughout the 3D structure in comparison to scaffolds prepared via bulk gelation without any macropores.

Although the extent of mineralization increased, XRD data shown in Figure 3.17 indicate that the presence of macropores had no effect on which $\mathrm{CaCO}_{3}$ morphology was formed or which polymorph was selected within the final $3 \mathrm{D}$ chitosan- $\mathrm{CaCO}_{3}$ composite scaffolds. Studies have shown that confinement affects the crystallization pathway, the orientation of crystallites and polymorph selection of minerals such as the stabilization of vaterite and amorphous calcium carbonate in confinement or the initial formation of amorphous calcium phosphate in between the collagen fibrils leading to hydroxyapatite formation in bone [4345]. However, in the case of 3D printed chitosan hydrogel-based scaffolds, although the presence of macropores led to a localized increase in the concentration of mineral ions in these regions, these did not affect the polymorph selection and calcium carbonate predominantly existed as calcite. XRD data for composite scaffolds with different macropore sizes $(1.5 \mathrm{~mm} \times 1.5 \mathrm{~mm}$ and $2 \mathrm{~mm} \times 2 \mathrm{~mm}$ macropores $)$ showed that the increase in the macropore size also did not induce any variation in the polymorph selection and calcium carbonate predominantly existed as calcite. 
(a) Mineralization in the presence of PAA (no macropores)

(b) Mineralization in the presence of PAA (1.5 $\mathrm{mm} \times 1.5 \mathrm{~mm}$ macropores)

(c) Mineralization in the presence of PAA (2.0 $\mathrm{mm} \times 2.0 \mathrm{~mm}$ macropores)

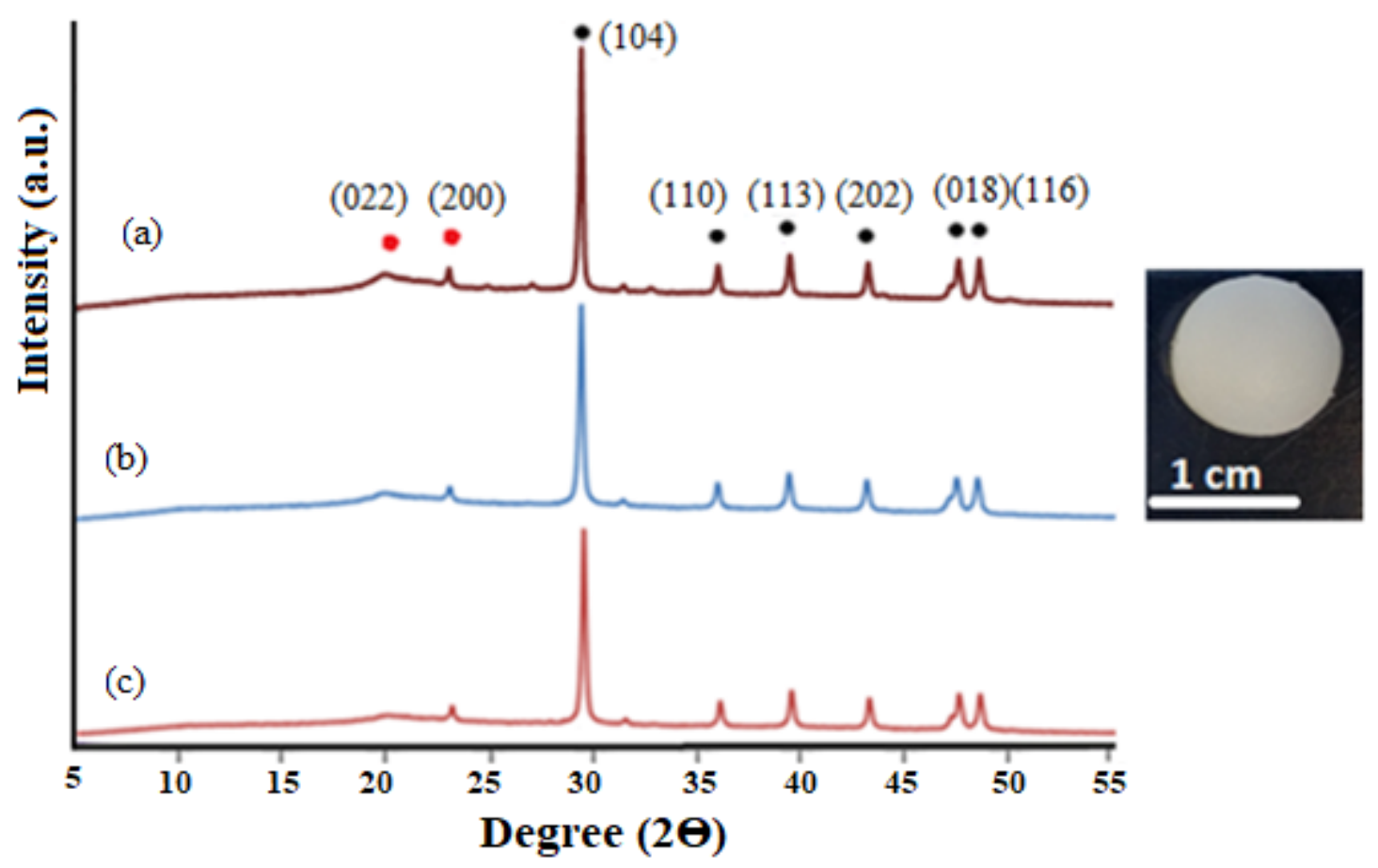

Figure 3.17: XRD plots for air-dried 3D chitosan hydrogel-based scaffolds with or without macropores (macropores introduced by the 3D printer; dimensions shown are of corresponding as-fabricated scaffolds in wet state) mineralized via the McGrath method (alternate soaking for $24 \mathrm{~h}$ in $0.5 \mathrm{M}$ precursor ion solutions + soaking in Kitano solution (1 week)) in the presence or absence of $2.5 \% \mathrm{w} / \mathrm{w}$ PAA with respect to the dry weight of chitosan. The peaks represented by black and red dots correspond to calcite and chitosan respectively. The optical image on the right is a chitosan hydrogel-based $3 D$ scaffold without any macropores.

The localized increase in the mineral concentration within the macropores and the resultant growth of crystallites on the exposed regions of the macropores affected the total porosity of the final composite structure in comparison to that of non-mineralized chitosan hydrogelbased scaffolds. Porosity calculations based on the reconstructed micro-CT scans of the airdried 3D composites developed via different mineralization techniques showed that the final porosity of the composites is slightly lower than that of the air-dried non-mineralized scaffolds - both developed from the chitosan hydrogel-based scaffolds with the same initial macropore dimensions in the wet state. Table 3.5 shows the percentage porosity of the airdried composites fabricated by the mineralization of chitosan hydrogel-based scaffolds with 
$(1.5 \mathrm{~mm} \times 1.5 \mathrm{~mm}$ macropores) in the wet state, via different methods in comparison to that of non-mineralized scaffolds.

\begin{tabular}{|c|c|}
\hline Mineralization technique & $\begin{array}{c}\text { Porosity calculated via } \\
\text { micro-CT (\%) }\end{array}$ \\
\hline No mineralization & $51.6 \pm 1.3$ \\
\hline $\begin{array}{c}\text { McGrath mineralization: alternate soaking in 0.5 M } \\
\text { precursor ion solutions (24 h) + Kitano (1 week) }\end{array}$ & $48.6 \pm 1.0$ \\
\hline $\begin{array}{c}\text { McGrath mineralization: alternate soaking in 0.25 M } \\
\text { precursor ion solutions (24 h) + Kitano (1 week) }\end{array}$ & $39.9 \pm 3.3$ \\
\hline $\begin{array}{c}\text { McGrath mineralization: alternate soaking in 0.1 M } \\
\text { precursor ion solutions (24 h) + Kitano (1 week) }\end{array}$ & $43.4 \pm 0.4$ \\
\hline $\begin{array}{c}\text { Soaking in Kitano solution (saturated CaCO } 3 \text { solution) } \\
(1 \text { week) }\end{array}$ & $43.1 \pm 3.2$ \\
\hline
\end{tabular}

Table 3.5: The total porosity of the final air-dried chitosan-CaCO 3 scaffolds fabricated by mineralization of chitosan hydrogel-based scaffolds with $1.5 \mathrm{~mm} \times 1.5 \mathrm{~mm}$ macropores (dimension in the wet state), via different mineralization methods compared to that of nonmineralized scaffolds.

Nacre inspired calcification has been reported on several different templates such as PVA, diazo-resins and PAA, poly (styrene)-block-poly (acrylic acid) block copolymer monolayer etc. [8, 46, 47]. There are also many studies on chitosan- $\mathrm{CaCO}_{3}$ composites which primarily focus on the morphology and polymorph selection of $\mathrm{CaCO}_{3}$ in the presence of different crystal growth modifiers, including work by Kato et al. who reported results for the fabrication of $3 \mathrm{D}$ chitosan- $\mathrm{CaCO}_{3}$ composites $[48,49]$. In latter work, reports showed effective synthetic replication of nacre in the form of multi-layered chitosan-calcium carbonate composites wherein thin layers of chitosan were spin coated and subsequently mineralized to obtain calcium carbonate crystallization within each layer, one at a time, in the presence of poly amino acids [10].

However, to the best of our knowledge, no work has been reported on in situ calcification/mineralization of 3D chitosan hydrogel-based scaffolds. This work shows that up to $40 \%$ (Table 3.5) mineralization can be achieved via in situ biomineralization of 
chitosan hydrogel-based 3D scaffolds using the McGrath method. The morphology of the resultant crystallites in the regions that were directly exposed to mineralization media was comparable to those reported in the literature for calcification of $2 \mathrm{D}$ chitin/chitosan films when using PAA as the crystal growth modifier. In between the printed layers, although the morphology was slightly different from the desire pancake-like structure, the micronsized calcium carbonate crystallites were still in the form of aggregates of nano-sized $\mathrm{CaCO}_{3}$ growing association with the hydrogel matrix. This kind of hierarchal organization within 3D biopolymer-based scaffolds, on a laboratory scale, has not been reported in the literature. Although the mineral distribution is not entirely uniform throughout the scaffold, the improved interaction between the mineral component and the biopolymer matrix is envisioned to improve the mechanical properties of the 3D scaffold, which will be further explored in Chapter 5. 


\subsection{Conclusion}

The mineralization achieved with 2D chitosan hydrogel-based films was successfully translated to 3D printed chitosan hydrogel-based scaffolds with some modification being required in the processing protocol for 3D scaffolds. The modified processing method was designed to ensure that the 3D scaffolds were sufficiently porous to facilitate diffusion when the scaffold is introduced into the mineralization media and hence increase the extent of mineralization throughout the 3D scaffold. SEM, EDS and XRD results showed that this was achieved via the new method and that the printed macropores further aided in increasing the depth of penetration of the minerals into the scaffold as compared to 3D scaffolds developed via bulk gelation of chitosan hydrogel, which had no macropores. This is attributed to the larger area available to interact with the mineralization solutions due to the presence of printed macropores as well as the layer-by-layer build of the scaffolds. However, these macropores did not affect the crystallization pathway or the polymorph selection of the mineral and calcium carbonate predominantly existed as calcite.

When 3D scaffolds were mineralized via the McGrath method in the absence of PAA, $\mathrm{CaCO}_{3}$ crystallites predominantly exhibited rhombohedra, spherical, rosette-like morphologies within the hydrogel matrix and $\mathrm{XRD}$ revealed that $\mathrm{CaCO}_{3}$ existed as calcite. Introduction of PAA as the crystal growth modifier led to the suppression of axial growth of the $\mathrm{CaCO}_{3}$ crystallites leading to the formation of nanosized crystals which then aggregate (while being entangled/associated with the polymer matrix) to form laterally growing pancake-like crystallites. The formation of such crystallites could be suggested as an effective interplay between the concentration of PAA and the mineral precursor ions available to interact with each other at different regions of the scaffold. $2.5 \%$ w/w PAA was chosen as a suitable concentration that leads to the formation of pancake-like crystallites in between the printed layers as well as the regions directly exposed to the mineralization media.

Each micron-sized $\mathrm{CaCO}_{3}$ crystallite was seen to grow in continued association with the organic matrix. In the regions directly exposed to the mineralization media (in the presence of low concentration of precursor ions), individual pancake-like crystallites are formed by oriented aggregation of numerous $\mathrm{CaCO}_{3}$ nanoplatelets oriented perpendicular to the organic hydrogel matrix, introducing a hierarchy in the composite structure. Since the modified mineralization protocol results in extensive mineralization of the $3 \mathrm{D}$ scaffold and 
hierarchal growth of $\mathrm{CaCO}_{3}$ in close association with the matrix, this protocol was chosen as the best protocol to mineralize 3D chitosan hydrogel-based scaffolds in this research.

Furthermore, when using the McGrath method for mineralization of the 3D chitosan hydrogel-based scaffolds, the effect of varying (1) the concentration of the crystal growth modifier (PAA); (2) the concentration of the mineralization solutions; and (3) the time period of exposure of the scaffold to the mineralization media on the morphology of the resultant $\mathrm{CaCO}_{3}$ crystallites and the extent of mineralization of the 3D hydrogel-based scaffold were investigated.

It was observed that different steps involved in the McGrath method can be modified in order to alter the degree of mineralization within the 3D chitosan hydrogel-based scaffold. Based on the results, it could be suggested that, the alternate soaking step in the McGrath mineralization method is a determinant step towards the formation of the final crystallites with the desired pancake-like morphology. Also, it was found that increasing the concentration and the period of exposure to the mineral precursor ion solutions during the alternate soaking step increases the extent of mineralization achieved throughout the 3D scaffold. An appropriate distribution of pancake-like crystallites in the regions exposed to the mineralized media as well as in between the printed layers of a 3D chitosan hydrogelbased scaffold was obtained when these scaffolds were mineralized via the McGrath method in the presence of $0.25 \mathrm{M}$ or $0.1 \mathrm{M}$ precursor ion solution. Based on SEM image analysis, the protocol that best replicates the "brick-mortar" nanostructure of nacre on a macroscale when using 3D chitosan hydrogel-based scaffolds was deduced as the McGrath mineralization of chitosan hydrogel-based scaffolds by $2 \mathrm{~h}$ alternating exposure to $0.25 \mathrm{M}$ mineral precursor ions followed by 3 days soaking in Kitano solution.

The results suggest that the McGrath mineralization method in the presence of PAA can be used to grow $\mathrm{CaCO}_{3}$ crystallites in close association with the chitosan hydrogel matrix in the 3D scaffolds, introducing hierarchy in the final composite structure. Although this method results in the lateral growth of $\mathrm{CaCO}_{3}$ crystallites throughout the scaffold, the size, morphology and uniformity in the distribution of the resultant crystallites were found to vary in different regions of the 3D scaffold. Hence, it can be deduced that translation of the nacre-like mineralization achieved on a $2 \mathrm{D}$ chitosan hydrogel-based template to $3 \mathrm{D}$ scaffolds is not straightforward and requires further development to optimise the mineral distribution in the material. 


\subsection{Bibliography}

1. Mi, H.-Y., X. Jing, M.R. Salick, T.M. Cordie, X.-F. Peng, and L.-S. Turng, Morphology, mechanical properties, and mineralization of rigid thermoplastic polyurethane/hydroxyapatite scaffolds for bone tissue applications: effects of fabrication approaches and hydroxyapatite size. Journal of Materials Science, 2014. 49(5): p. 2324-2337.

2. Zhu, X., N. Liu, M.J. Yaszemski, and L. Lu, Effects of composite formulation on mechanical properties of biodegradable poly (propylene fumarate)/bone fiber scaffolds. International Journal of Polymer Science, 2010. 2010.

3. Zhao, H., L. Ma, C. Gao, and J. Shen, Fabrication and properties of mineralized collagen-chitosan/hydroxyapatite scaffolds. Polymers for Advanced Technologies, 2008. 19(11): p. 1590-1596.

4. Campana, V., G. Milano, E. Pagano, M. Barba, C. Cicione, G. Salonna, W. Lattanzi, and G. Logroscino, Bone substitutes in orthopaedic surgery: from basic science to clinical practice. Journal of Materials Science: Materials in Medicine, 2014. 25(10): p. 2445-2461.

5. Gunn, J.M., J. Rekola, J. Hirvonen, and A.J. Aho, Comparison of the osteoconductive properties of three particulate bone fillers in a rabbit model: allograft, calcium carbonate (Biocoral®) and S53P4 bioactive glass. Acta Odontologica Scandinavica, 2013. 71(5): p. 1238-1242.

6. Levengood, S.K.L. and M. Zhang, Chitosan-based scaffolds for bone tissue engineering. Journal of Materials Chemistry B, 2014. 2(21): p. 3161-3184.

7. Manjubala, I., S. Scheler, J. Bössert, and K.D. Jandt, Mineralisation of chitosan scaffolds with nano-apatite formation by double diffusion technique. Acta Biomaterialia, 2006. 2(1): p. 75-84.

8. Wei, H., N. Ma, F. Shi, Z. Wang, and X. Zhang, Artificial nacre by alternating preparation of layer-by-layer polymer films and $\mathrm{CaCO}_{3}$ strata. Chemistry of Materials, 2007. 19(8): p. 1974-1978.

9. Küther, J., G. Nelles, R. Seshadri, M. Schaub, H.J. Butt, and W. Tremel, Templated Crystallisation of Calcium and Strontium Carbonates on Centred Rectangular SelfAssembled Monolayer Substrates. Chemistry-A European Journal, 1998. 4(9): p. 18341842. 
10. Kato, T., Polymer/Calcium Carbonate Layered Thin-Film Composites. Advanced Materials, 2000. 12(20): p. 1543-1546.

11. Xu, G., N. Yao, I.A. Aksay, and J.T. Groves, Biomimetic synthesis of macroscopicscale calcium carbonate thin films. Evidence for a multistep assembly process. Journal of the American Chemical Society, 1998. 120(46): p. 11977-11985.

12. Sugawara, A., T. Ishii, and T. Kato, Self-Organized Calcium Carbonate with Regular Surface-Relief Structures. Angewandte Chemie, 2003. 115(43): p. 5457-5461.

13. Lu, C., L. Qi, J. Ma, H. Cheng, M. Zhang, and W. Cao, Controlled growth of micropatterned, oriented calcite films on a self-assembled multilayer film. Langmuir, 2004. 20(18): p. 7378-7380.

14. Serizawa, T., T. Tateishi, D. Ogomi, and M. Akashi, Deposition of calcium carbonate disks on polyelectrolyte multilayer matrices by the alternate soaking process. Journal of Crystal Growth, 2006. 292(1): p. 67-73.

15. Swain, S.K., S. Dash, S.K. Kisku, and R.K. Singh, Thermal and oxygen barrier properties of chitosan bionanocomposites by reinforcement of calcium carbonate nanopowder. Journal of Materials Science \& Technology, 2014. 30(8): p. 791-795.

16. Suzuki, M., Y. Dauphin, L. Addadi, and S. Weiner, Atomic order of aragonite crystals formed by mollusks. CrystEngComm, 2011. 13(22): p. 6780-6786.

17. Ren, D., Q. Feng, and X. Bourrat, Effects of additives and templates on calcium carbonate mineralization in vitro. Micron, 2011. 42(3): p. 228-245.

18. Falini, G., S. Albeck, S. Weiner, and L. Addadi, Control of aragonite or calcite polymorphism by mollusk shell macromolecules. Science, 1996. 271(5245): p. 67.

19. He, F., W. Ren, X. Tian, W. Liu, S. Wu, and X. Chen, Comparative study on in vivo response of porous calcium carbonate composite ceramic and biphasic calcium phosphate ceramic. Materials Science and Engineering: C, 2016. 64: p. 117-123.

20. Altay, E., T. Shahwan, and M. Tanoğlu, Morphosynthesis of $\mathrm{CaCO}_{3}$ at different reaction temperatures and the effects of PDDA, CTAB, and EDTA on the particle morphology and polymorph stability. Powder Technology, 2007. 178(3): p. 194-202.

21. Declet, A., E. Reyes, and O. Suárez, Calcium carbonate precipitation: a review of the carbonate crystallization process and applications in bioinspired composites. Reviews on Advanced Materials Science, 2016. 44(1).

22. Murphy, W., J. Black, and G.W. Hastings, Handbook of biomaterial properties. 2016: Springer. 
23. Costa-Pinto, A.R., R.L. Reis, and N.M. Neves, Scaffolds based bone tissue engineering: the role of chitosan. Tissue Engineering Part B: Reviews, 2011. 17(5): p. 331-347.

24. Geng, L., Y. Wong, D. Hutmacher, W. Feng, H. Loh, and J. Fuh, Rapid Prototyping of 3D Scaffolds for Tissue Engineering Using a Four-Axis Multiple-Dispenser Robotic System. Dept. of Mechanical Engineering, National University of Singapore, Singapore, 2003.

25. Barthelat, F., C.-M. Li, C. Comi, and H.D. Espinosa, Mechanical properties of nacre constituents and their impact on mechanical performance. Journal of Materials Research, 2006. 21(8): p. 1977-1986.

26. Currey, J.D., P. Zioupos, D. Peter, and A. Casinos, Mechanical properties of nacre and highly mineralized bone. Proceedings of the Royal Society of London B: Biological Sciences, 2001. 268(1462): p. 107-111.

27. Zhang, S. and K. Gonsalves, Synthesis of calcium carbonate-chitosan composites via biomimetic processing. Journal of applied polymer science, 1995. 56(6): p. 687-695.

28. Munro, N.H., D.W. Green, A. Dangerfield, and K.M. McGrath, Biomimetic mineralisation of polymeric scaffolds using a combined soaking and Kitano approach. Dalton Transactions, 2011. 40(36): p. 9259-9268.

29. Fan, H., L. Wang, K. Zhao, N. Li, Z. Shi, Z. Ge, and Z. Jin, Fabrication, mechanical properties, and biocompatibility of graphene-reinforced chitosan composites. Biomacromolecules, 2010. 11(9): p. 2345-2351.

30. Lazzeri, A., S.M. Zebarjad, M. Pracella, K. Cavalier, and R. Rosa, Filler toughening of plastics. Part 1-the effect of surface interactions on physico-mechanical properties and rheological behaviour of ultrafine $\mathrm{CaCO}_{3} / \mathrm{HDPE}$ nanocomposites. Polymer, 2005. 46(3): p. 827-844.

31. Mann, S., Biomineralization: principles and concepts in bioinorganic materials chemistry. Vol. 5. 2001: Oxford University Press on Demand.

32. Chen, M.H., L.L. Wang, J.J. Chung, Y.-H. Kim, P. Atluri, and J.A. Burdick, Methods to assess shear-thinning hydrogels for application as injectable biomaterials. ACS Biomaterials Science \& Engineering, 2017. 3(12): p. 3146-3160.

33. Yuwono, V.M., N.D. Burrows, J.A. Soltis, and R.L. Penn, Oriented aggregation: formation and transformation of mesocrystal intermediates revealed. Journal of the American Chemical Society, 2010. 132(7): p. 2163-2165. 
34. Song, R.Q. and H. Cölfen, Mesocrystals—ordered nanoparticle superstructures. Advanced Materials, 2010. 22(12): p. 1301-1330.

35. Wang, S.-F., L. Shen, W.-D. Zhang, and Y.-J. Tong, Preparation and mechanical properties of chitosan/carbon nanotubes composites. Biomacromolecules, 2005. 6(6): p. 3067-3072.

36. Munro, N.H. and K.M. McGrath, Hydrogels as biomimetic mineralisation scaffolds. Bioinspired, Biomimetic and Nanobiomaterials, 2012. 1(1): p. 26-37.

37. Kulak, A.N., P. Iddon, Y. Li, S.P. Armes, H. Cölfen, O. Paris, R.M. Wilson, and F.C. Meldrum, Continuous structural evolution of calcium carbonate particles: a unifying model of copolymer-mediated crystallization. Journal of the American Chemical Society, 2007. 129(12): p. 3729-3736.

38. Munro, N.H. and K.M. McGrath, Biomimetic approach to forming chitin/aragonite composites. Chemical Communications, 2012. 48(39): p. 4716-4718.

39. Nguyen, A.T., S.R. Sathe, and E.K. Yim, From nano to micro: topographical scale and its impact on cell adhesion, morphology and contact guidance. Journal of Physics: Condensed Matter, 2016. 28(18): p. 183001.

40. Munro, N.H. and K.M. McGrath, How important is polyelectrolyte complex formation in biomimetic mineralisation? Manipulation via alcohol addition. Dalton Transactions, 2013. 42(23): p. 8259-8269.

41. Chia, H.N. and B.M. Wu, Recent advances in $3 D$ printing of biomaterials. Journal of Biological Engineering, 2015. 9(1): p. 4.

42. Murakami, F.S., P.O. Rodrigues, C.M.T.d. Campos, and M.A.S. Silva, Physicochemical study of $\mathrm{CaCO}_{3}$ from egg shells. Food Science and Technology (Campinas), 2007. 27(3): p. 658-662.

43. Stephens, C.J., S.F. Ladden, F.C. Meldrum, and H.K. Christenson, Amorphous calcium carbonate is stabilized in confinement. Advanced Functional Materials, 2010. 20(13): p. 2108-2115.

44. Wang, Y.-W., H.K. Christenson, and F.C. Meldrum, Confinement increases the lifetimes of hydroxyapatite precursors. Chemistry of Materials, 2014. 26(20): p. 58305838.

45. Nudelman, F., K. Pieterse, A. George, P.H. Bomans, H. Friedrich, L.J. Brylka, P.A. Hilbers, G. de With, and N.A. Sommerdijk, The role of collagen in bone apatite formation in the presence of hydroxyapatite nucleation inhibitors. Nature Materials, 2010. 9(12): p. 1004-1009. 
46. Gong, H., M. Pluntke, O. Marti, P. Walther, L. Gower, H. Cölfen, and D. Volkmer, Multilayered $\mathrm{CaCO}_{3}$ /block-copolymer materials via amorphous precursor to crystal transformation. Colloids and Surfaces A: Physicochemical and Engineering Aspects, 2010. 354(1): p. 279-283.

47. Finnemore, A., P. Cunha, T. Shean, S. Vignolini, S. Guldin, M. Oyen, and U. Steiner, Biomimetic layer-by-layer assembly of artificial nacre. Nature Communications, 2012. 3: p. 966.

48. Zhang, S. and K. Gonsalves, Chitosan-calcium carbonate composites by a biomimetic process. Materials Science and Engineering: C, 1995. 3(2): p. 117-124.

49. Kotachi, A., T. Miura, and H. Imai, Polymorph Control of Calcium Carbonate Films in a Poly (acrylic acid) - Chitosan System. Crystal Growth \& Design, 2006. 6(7): p. 1636-1641. 


\section{3D Printed Chitosan-Calcium Carbonate Hydrogel-based Composites}

\subsection{Introduction}

While there are examples of 3D printed biopolymer-based composites being fabricated via complex co-precipitation or biomimetic methods, such as the mineralization method discussed in Chapter 3, this is not the only method used to fabricate such composites. Other methods reported in the literature include electrochemical deposition [1]; soaking in simulated body fluids [2]; and by dispersing the mineral particles in the polymer matrix via mechanical mixing or ultra-sonication and then freeze-drying/lyophilization (also known as thermally-induced phase separation) $[3,4]$.

In the context of extrusion-based 3D bio-printing, it is straightforward to develop 3D composites by using composite hydrogel-based inks that are developed via simply mechanically blending the mineral phases within the organic phase, instead of following complex processes involving in situ biomineralization or co-precipitation of the mineral phase within the pure polymer-based scaffold structures [5]. Although the former is a very straightforward technique compared to the latter, literature suggests that the nature of the interaction between the mineral and the organic phase, which is a determinant factor in the mechanical properties of the final composite structure, is essentially weak in composites based on mechanically blended organic-inorganic polymer composites. This eventually results in the mechanical strength of such structures being much lower than those fabricated via biomimetic techniques [6,7]. This is because the biomimetic techniques promote the chemical bonding between the mineral particles and the polymer chains at a molecular level. An example highlighting this approach is a study reported by Hu et al. whereby chitosannHA composite rods for bone fracture fixation applications were fabricated via in situ hybridization (biomimetic technique) and also by the blending method. It was found that the former resulted in (1) uniform distribution of the mineral phase within the organic phase; (2) the resultant particles were nanosized; and (3) the final air-dried composites exhibited a higher bending strength ( $80 \mathrm{MPa}$ and modulus of $3.9 \mathrm{GPa}$ ) than those fabricated via the blending method (compressive strength of $68 \mathrm{MPa}$ and modulus of $3.2 \mathrm{GPa}$ ). It was 
suggested that the higher mechanical resistance exhibited could be attributed to the enhanced particles-matrix interaction observed in the case of those fabricated via the biomimetic technique [8]. It is noted however that biomimetic fabrication techniques are very laborious and time-consuming. Thus, it is important to explore other fabrication techniques that may result in composites with similar properties and is simpler in comparison.

Hence, the mechanical blending-based fabrication process presents potential advantages as well as disadvantages over the approach presented in Chapter 3. The purpose of the investigation presented here is to determine if it is a viable alternative fabrication protocol. Thus, the fabrication of $3 \mathrm{D}$ printed chitosan-calcium carbonate composites using printing inks based on chitosan hydrogels preloaded with calcium carbonate crystallites is presented here. In particular, the practical advantages/disadvantages of using a blended hydrogel (chitosan-calcium carbonate hydrogel) as the printing ink rather than a pure chitosan hydrogel are discussed.

It was shown in Chapter 3 that biomimetic mineralization techniques such as the McGrath mineralization method (when crystal growth modifiers such as polyacrylic acid (PAA) are present) result in the formation of calcium carbonate nanoparticles which are distinctly and intimately associated with the hydrogel matrix. These nanoparticles aggregate in a controllable manner resulting in the formation of larger micron-sized chitosan- $\mathrm{CaCO}_{3}$ composite particles within the hydrogel matrix. Under appropriate fabrication conditions, platelet-like aggregates that laterally conform to the organic matrix are generated in similarity to the structure of nacre. It is envisioned that the enhanced interfacial interaction achieved using this fabrication technique will improve the mechanical properties of the composites.

A major disadvantage of using the mechanical blending technique is the resultant lack of a strong chemical or physical interaction between the mineral and the organic phase in the final composite and a thus reduced mechanical strength. This can be improved by modifying the morphology, size and orientation of the crystallites within the organic matrix [9-11]. Typically, nanoparticles are chosen over micron-sized particles as the mineral phase in an effort to improve the mechanical properties of the composite. However, nanoparticles have higher surface energy which often leads to random aggregation when blended together, particularly in the presence of a charged polymeric system, which negatively impacts the final material properties. Such aggregates and the resultant non-uniform dispersion of the 
mineral phase within the polymer matrix leads to an increase in the number of interfaces that act as prospective fracture surfaces aiding in crack propagation when the material is stressed [12]. Typically, such unpremeditated aggregation can be overcome by surface functionalization of the nanoparticles, which improves the tendency of the nanoparticles to bind to the polymeric phase during the blending process rather than aggregate and thus aid also in uniform distribution throughout the polymeric matrix [13]. In the fabrication process described in this Chapter, the calcium carbonate which is added directly to the chitosan hydrogel to form the blended printing ink, is not nanosized, hence, the variability in the morphology and size of the mineral phase and its nature of interaction with the polymer matrix at each of the different stages of the fabrication process and in the final composite material were investigated.

The various characteristics of the alternative fabrication process used and the thus fabricated 3D porous chitosan-calcium carbonate composites explored include:

(a) the tunability of the morphology of the resultant crystallites within the organic matrix (crystallite morphologies within the composites at different stages of the fabrication process and the final morphologies within the composites);

(b) the effect of introducing the crystal growth modifier, PAA, into the blended ink in an effort to modify the structure of the resultant crystallites to the desired pancakelike structure; and

(c) the nature of the interaction between the crystallites and the organic matrix in the presence or absence of PAA. 


\subsection{Materials and methods}

Chemicals used in this study were procured from the same manufactures as described in the materials section of Chapter 3 .

\subsubsection{Chitosan-calcium carbonate hydrogel preparation}

Chitosan-calcium carbonate hydrogels $\left(\mathrm{Ch}-\mathrm{CaCO}_{3}\right)$ to be used as the printing ink in our custom designed 3D printer were prepared by mixing different quantities of powdered $\mathrm{CaCO}_{3}$ (as obtained from the manufacturer, $5.2 \pm 3 \mu \mathrm{m}$, Figure $4.4(\mathrm{k})$ ) with $5 \% \mathrm{w} / \mathrm{v}$ chitosan in purified water in the presence of acetic acid under a constant stirring at $700 \mathrm{rpm}$ for $6 \mathrm{~h}$ until a uniform gel was obtained. The amount of calcium carbonate added was varied from $10 \%$ to $50 \%$ w/w w.r.t $5 \%$ w/v chitosan. Initially, $2 \%$ v/v acetic acid was used to prepare the blended inks irrespective of the amount of calcium carbonate added. However, it was observed that the resultant gels were highly viscous and were unsuitable for use as the printing ink in our custom-designed 3D printer when using an extrusion nozzle with a $0.42 \mathrm{~mm}$ internal diameter. The viscosity of the gels can be modified by changing the acidity of the solution used to prepare the hydrogels. By undertaking a rheological investigation of the gels (discussed in Section 4.3.1) as a function of acidity, the amount of acetic acid required to achieve the hydrogel viscosity that ensured good printability was ascertained for each calcium carbonate loading. The rheological properties of all $\mathrm{Ch}-\mathrm{CaCO}_{3}$ blended hydrogels were studied using the same parameters and instrument as described in Chapter 2, Section 2.2.2.

\subsubsection{D printing}

The $\mathrm{Ch}-\mathrm{CaCO}_{3}$ blended hydrogel-based printing inks were used in conjunction with the same CAD model as described in Chapter 2. The same printing parameters were used. Scaffolds with $1.5 \mathrm{~mm} \times 1.5 \mathrm{~mm}$ macropores were fabricated at $6 \mathrm{~mm} \mathrm{~s}^{-1}$ printing speed. $1.5 \% \mathrm{w} / \mathrm{v}(0.37 \mathrm{M})$ to $1 \mathrm{M}$ ethanolic $\mathrm{NaOH}(70: 30 \mathrm{v} / \mathrm{v} \mathrm{NaOH}$ : ethanol) solution was used to laminate layers of printed gel in order to develop a stable 3D structure. The as-fabricated scaffolds were then rested in $1 \mathrm{M} \mathrm{NaOH}$ for $24 \mathrm{~h}$ to ensure complete gelation has occurred. The scaffolds were then washed with purified water until neutral $\mathrm{pH}$ was achieved and were stored in purified water until further characterization was undertaken.

All composites were air-dried prior to characterization via electron microscopy (SEM), Xray diffraction (XRD) and thermogravimetric analysis (TGA) unless otherwise mentioned. 
Structural and compositional analysis of the 3D scaffolds was performed using SEM and XRD. TGA was used to study the thermal decomposition behaviour of the $\mathrm{Ch}-\mathrm{CaCO}_{3}$ blended inks and the final composites. The sample preparation techniques and experimental parameters used for each of the characterization techniques were the same as described in Chapter 3. 


\subsection{Results and discussion}

In this Chapter, the advantages and disadvantages of fabricating 3D chitosan- $\mathrm{CaCO}_{3}$ composites using chitosan hydrogel-based inks preloaded with calcium carbonate crystallites (blended hydrogels) as an alternative method to the biomimetic mineralization technique discussed in Chapter 3 are investigated.

\subsubsection{Rheology of $\mathrm{Ch}-\mathrm{CaCO}_{3}$ gels}

The first critical step was to ascertain the ease with which the 3D printing could be undertaken using the custom-designed 3D printer when using the blended hydrogels as the printing ink. This was investigated via analysis of their rheological properties under shear stress based on the data shown in Chapter 2. Rheological analysis showed that the viscosity of the chitosan hydrogels $(5 \% \mathrm{w} / \mathrm{v}$ in $2 \% \mathrm{v} / \mathrm{v}$ acetic acid solution) increased with the percentage of $\mathrm{CaCO}_{3}$ added. This is consistent with reported literature which suggests that the viscosity of hydrogel increases as a function of the concentration of filler particles [14].

Figure 4.1 shows the log-log plot of the variation of viscosity of $5 \% \mathrm{w} / \mathrm{v}$ chitosan hydrogels (prepared in the presence of $2 \% \mathrm{v} / \mathrm{v}$ acetic acid solution ( $\mathrm{pH} 2.8)$ ) loaded with increasing percentage of $\mathrm{CaCO}_{3}$ under increasing shear stress. The following were deduced from this plot:

(a) at low shear rates, such as those present at the initiation of extrusion, the viscosity of the composite hydrogels is significantly higher than that of pure chitosan hydrogels. Consider for example a shear rate of $0.1 \mathrm{~s}^{-1}$, the viscosity of blended inks is $\sim 13$ to 100 times higher than that of the pure chitosan hydrogel. Even in the case of blended hydrogels with $10 \%$ w/w of calcium carbonate (w.r.t to $5 \%$ w/v chitosan), the minimum amount of $\mathrm{CaCO}_{3}$ added in this investigation, an increase of more than an order of magnitude was observed; and

(b) at higher shear rates, similar to that experienced by the hydrogel at the tip of a 0.42 mm extrusion nozzle $\left(72 \pm 7 \mathrm{~s}^{-1}\right)$, the viscosity of the composite hydrogels was comparable to that of the pure chitosan hydrogel and in the range found to be used as printing ink in our custom designed 3D printer when using a $0.42 \mathrm{~mm}$ extrusion nozzle (refer to Chapter 2).

The observed higher viscosity of the composite hydrogels at low shear rates suggest that a very high pressure is required to initiate the flow of such hydrogels during the printing 
process before being extruded as a continuous strand through a $0.42 \mathrm{~mm}$ nozzle (such high pressures exceed what is possible with the custom-designed printer used here). In comparison, it can be seen that $5 \% \mathrm{w} / \mathrm{v}$ chitosan hydrogels (in $2 \% \mathrm{v} / \mathrm{v}$ acetic acid solution) require a much lower pressure to initiate flow and thus be extruded through a $0.42 \mathrm{~mm}$ nozzle. Thus, it can be deduced that in order for a composite printing ink to be suitable in our custom-designed 3D printer when using a $0.42 \mathrm{~mm}$ extrusion nozzle and the previously established printing parameters (refer to Chapter 2), both its viscosity and flow properties at both low and high shear rates must be comparable to that of $5 \% \mathrm{w} / \mathrm{v}$ chitosan hydrogels (in $2 \% \mathrm{v} / \mathrm{v}$ acetic acid solution). Furthermore, as different inks can be used for a particular extrusion-based printer, it is critically important to understand the flow behaviour required in order to achieve reproducible and well-formed prints.

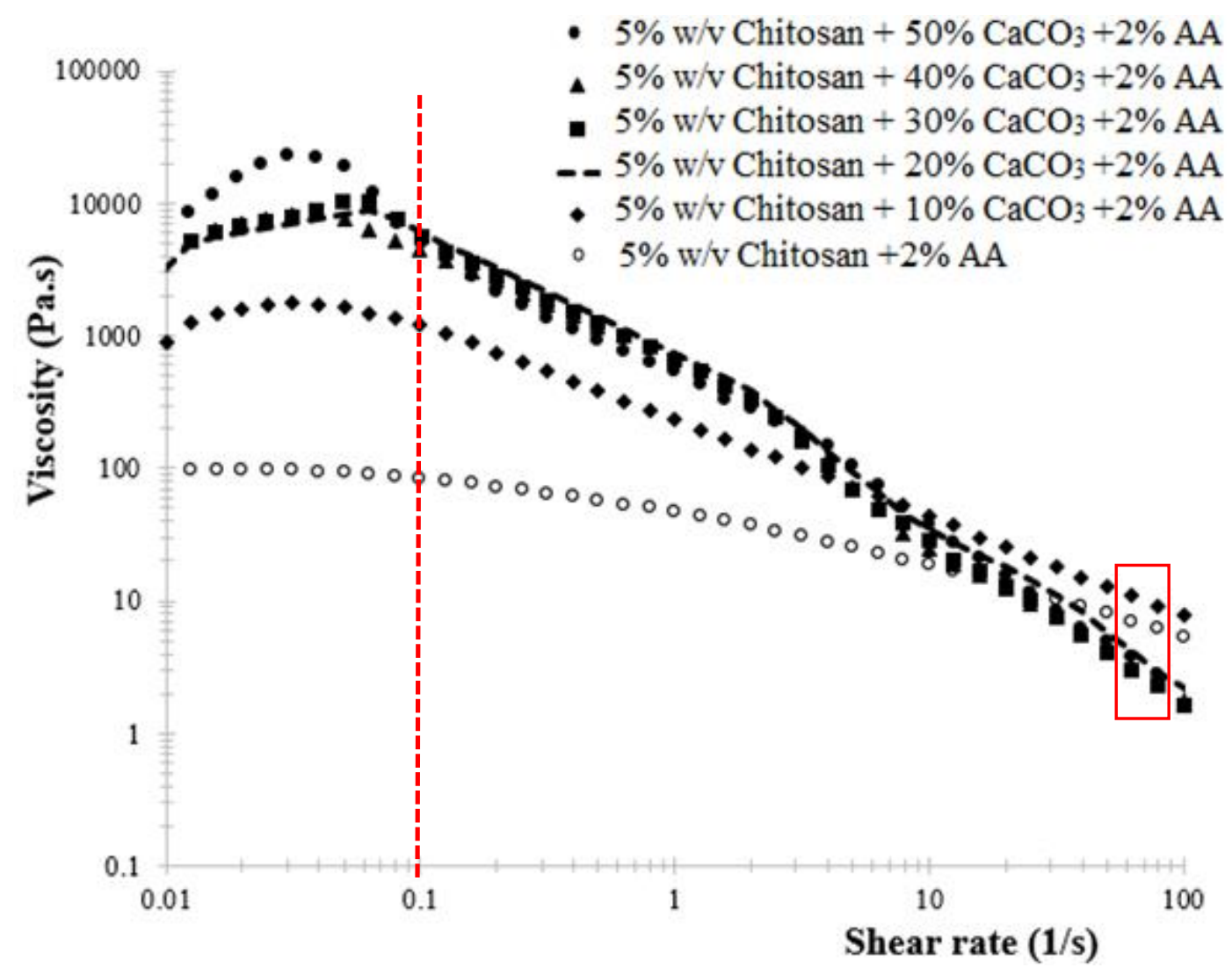

Figure 4.1: Log-log plot of the variation of viscosity as a function of the shear rate for a series of $5 \% \mathrm{w} / \mathrm{v}$ chitosan hydrogels prepared in the presence of $2 \% \mathrm{v} / \mathrm{v}$ acetic acid solution, with increasing percentage of added $\mathrm{CaCO}_{3}$. The red dotted line represents the viscosity at $0.1 \mathrm{~s}^{-1}$ strain and the region marked with the red box represents the approximate shear rate experienced at the printer extrusion nozzle tip $\left(72 \pm 7 s^{-1}\right)$ as described in Chapter 2. 
It was also observed that large quantities of undissolved chitosan particles were retained after the blending process, particularly as the percentage of the added $\mathrm{CaCO}_{3}$ was increased when $2 \% \mathrm{v} / \mathrm{v}$ acetic acid was used. These were visible as white flakes within the hydrogel. Hence, apart from the resultant high viscosity, such large particles will clog the extrusion nozzle during the printing process. This is not desirable since it was hoped that some dissolution of the micron-sized $\mathrm{CaCO}_{3}$ particles would occur, resulting in the formation of the preferred nano-sized particles which would consequently improve the strength of the interaction between the mineral and organic phases of the final composite.

In order to modify the viscosity and the flow behaviour of the blended hydrogels such that the final ink could be extruded through a $0.42 \mathrm{~mm}$ extrusion nozzle as a continuous strand, a critical requirement for printing, the concentration of acetic acid added to prepare the Ch$\mathrm{CaCO}_{3}$ blended hydrogel ink was increased. By reducing the $\mathrm{pH}$ of the system, and in turn enhancing the solubility of all components, the viscosity of the resultant hydrogel is reduced, and the flow behaviour was seen to conform more closely to that of $5 \%$ w/v chitosan hydrogels (in $2 \% \mathrm{v} / \mathrm{v}$ acetic acid solution), which was successfully used as a printing ink in our 3D printer when using $0.42 \mathrm{~mm}$ extrusion nozzle. Accordingly, chitosan hydrogels with $10-30 \% \mathrm{w} / \mathrm{w} \mathrm{CaCO}_{3}$ added were prepared in the presence of $5 \% \mathrm{v} / \mathrm{v}$ acetic acid $(\mathrm{pH} 2.8)$ and those with $40-50 \% \mathrm{w} / \mathrm{w} \mathrm{CaCO}_{3}$ added were prepared in the presence of $10 \% \mathrm{v} / \mathrm{v}$ acetic acid ( $\mathrm{pH}$ 2.4). At these $\mathrm{pH}$ values, white flakes (undissolved chitosan) were no longer visible to the naked eye.

Figure 4.2 shows the resulting $\log$-log plot of the variation of viscosity with shear rate for the modified $\mathrm{Ch}-\mathrm{CaCO}_{3}$ blended hydrogels with varying percentage of added $\mathrm{CaCO}_{3}$. After modifying the $\mathrm{pH}$ of the system, it can be observed that trend of the change in the viscosity with shear rate for all blended hydrogels is now comparable to that observed for the $5 \% \mathrm{w} / \mathrm{v}$ chitosan hydrogel system described in Chapter 3 that was able to be successfully used as the printing ink in our custom designed 3D printer when using an extrusion nozzle with 0.42 mm internal diameter.

Figure 4.3 shows the log-log plot of the variation in storage $\left(\mathrm{G}^{\prime}\right)$ and loss modulus $\left(\mathrm{G}^{\prime \prime}\right)$ of modified $\mathrm{Ch}-\mathrm{CaCO}_{3}$ hydrogels with varying percentage of $\mathrm{CaCO}_{3}$ added as a function of increasing oscillating frequency. Similar to the trend observed for chitosan hydrogel, at low frequencies $\mathrm{G}^{\prime}>\mathrm{G}^{\prime \prime}$ indicating predominantly viscous behaviour and then at higher frequencies $\mathrm{G}^{\prime}>\mathrm{G}^{\prime \prime}$ indicating a switch to elastic behaviour. The crossover point, where $\mathrm{G}^{\prime}=\mathrm{G}^{\prime \prime}$, was approximately $13 \mathrm{~Hz}$ for all the composite hydrogels. 


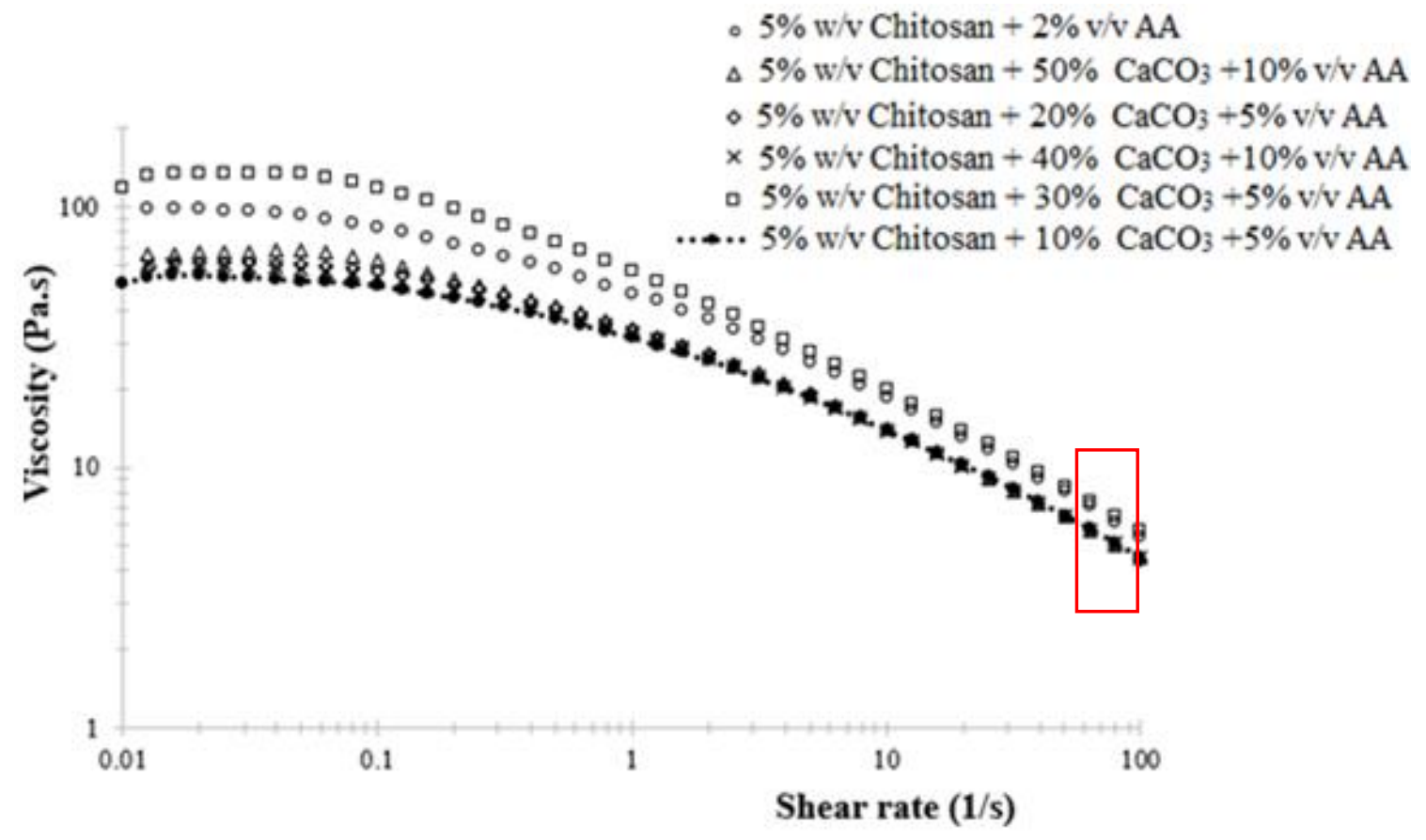

Figure 4.2: Log-log plot of the variation of viscosity as a function of the shear rate for a series of modified $\mathrm{Ch}-\mathrm{CaCO}$ blended hydrogels with varying percentage of added $\mathrm{CaCO}_{3}$ and acetic acid. The region marked with the red box represents the approximate shear rate experienced at the tip of the extrusion nozzle $\left(72 \pm 7 s^{-1}\right)$ as described in Chapter 2.

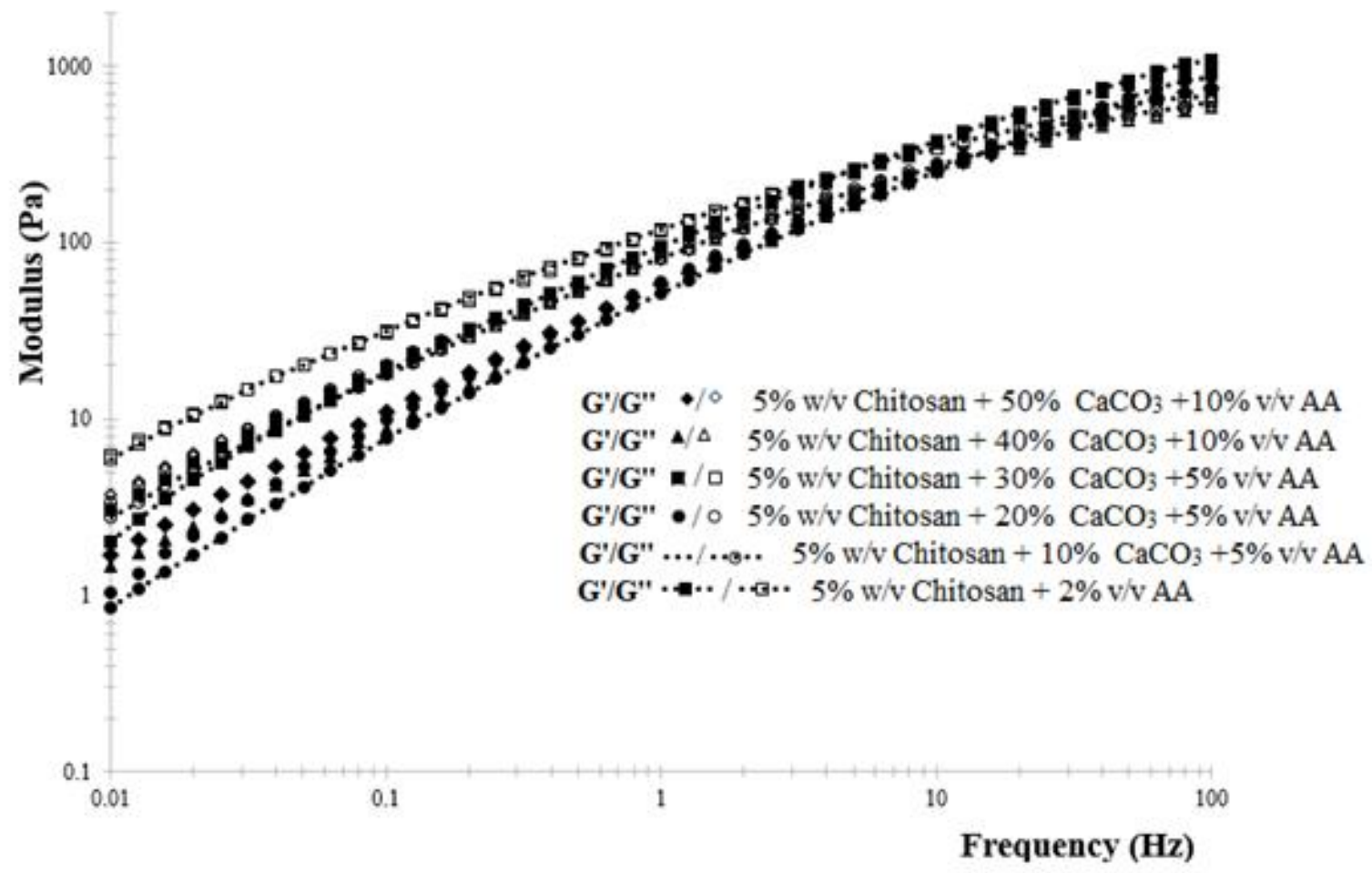

Figure 4.3: Log-log plot of the variation in the storage $\left(G^{\prime}\right)$ and loss moduli $\left(\mathrm{G}^{\prime \prime}\right)$ as a function of frequency $(\mathrm{Hz})$ for a series of the modified $\mathrm{Ch}-\mathrm{CaCO}_{3}$ hydrogels with varying percentage of added $\mathrm{CaCO}_{3}$ and acetic acid. 


\subsubsection{D printing using $\mathrm{Ch}-\mathrm{CaCO}_{3}$ blended inks}

The CAD model and the printing parameters used in this study are the same as those described in Chapter 2. However, the modifications required to improve the printability of the $\mathrm{Ch}-\mathrm{CaCO}_{3}$ blended hydrogels also resulted in some alternations in the 3D printing process being required in comparison to when pure chitosan hydrogels were used as the printing ink. Specifically, a higher concentration of ethanolic $\mathrm{NaOH}(1.5 \%$ w/v-1 M NaOH$)$ was required to partially deprotonate the printed $\mathrm{Ch}-\mathrm{CaCO}_{3}$ layers, such that the layers adhere to each other. This is due to the increased concentration of acetic acid used in the printing ink. The printed scaffolds were then rested in $1 \mathrm{M} \mathrm{NaOH}$ to promote complete gelation of the chitosan hydrogel. This process resulted in stable $3 \mathrm{D}$ chitosan- $\mathrm{CaCO}_{3}$ composites which are visually opaque.

While it is seemingly easier to fabricate $3 \mathrm{D}$ chitosan- $\mathrm{CaCO}_{3}$ composites by using a blended hydrogel as the printing ink instead of the using the two-step fabrication process described in Chapter 3, it is necessary to understand in detail the physical properties of the 3D composites thus obtained. The properties of the final 3D material initially analysed were:

(a) morphology of the resultant calcium carbonate crystallites within the composite; and

(b) the nature of the interaction between the chitosan hydrogel matrix and the calcium carbonate crystallites.

\subsubsection{Morphology of the calcium carbonate crystallites in $\mathrm{Ch}-\mathrm{CaCO}_{3}$ composites}

Tuning the morphology of the calcium carbonate particles and improving their interaction with the organic matrix are two important considerations in this research. The two-step chitosan- $\mathrm{CaCO}_{3}$ composite fabrication process, involving the $3 \mathrm{D}$ printing of chitosan hydrogel-based scaffolds and then biomimetic mineralization via the McGrath method (in the presence of PAA), offers considerable control over the morphology of the resultant crystallites. The morphology can be tuned by varying the concentrations of the precursor mineral ions, the concentration of crystal growth modifier (PAA) and the period of exposure to the mineralization media. One of the outcomes from such modifications is achieving hierarchal growth of the $\mathrm{CaCO}_{3}$ crystallites, which are also in relation with the organic matrix; specifically, these are in the form of laterally grown pancake-like crystallites formed via aggregation of nanosized calcium carbonate crystallites (mostly observed to form in between the printed layers) as described in Chapter 3. However, due to the many variable parameters in the fabrication process, fine-tuning of the morphology of the resultant 
crystallites to always achieve the desired pancake-like structure, throughout the 3D scaffold (in between the layers and the external surface) was not straightforwardly possible. This remains a limitation of the two-step fabrication process and further work is required to optimize these properties. The single-step fabrication technique offers an alternate route for manipulation of the $\mathrm{CaCO}_{3}$ morphology.

The single-step fabrication process is relatively an easier technique to develop 3D composites, however, similar to the system described in Chapter 3, there are numerous variables involved in this technique that can affect the tunability of the resultant crystallite morphology. It is well known that variables including the concentration of acetic acid used to develop individual printing inks and the subsequent variation in the concentration of ethanolic $\mathrm{NaOH}$ required to laminate the layers, which can be broadly considered as $\mathrm{pH}$ variation of the solution, affect the size, morphology and precipitation kinetics of calcium carbonate crystallite formation temperature [15] .

Furthermore, since this technique is based on the mechanical blending of components, the presence of a polymeric chain, herein chitosan and/or PAA, will modify and may restrict the extent of the interaction of the added $\mathrm{CaCO}_{3}$ with the different solutions (with different $\mathrm{pH})$ used in the fabrication process. This may result in the formation of final $\mathrm{CaCO}_{3}$ structures that are different from those obtained in the absence of the polymer in otherwise identical dissolution/nucleation environments. Therefore, in the first instance, it was necessary to understand the evolution of the $\mathrm{CaCO}_{3}$ morphology in the presence of chitosan hydrogel during the two major stages involved in the composite fabrication process; i.e. preparation of blended hydrogel and post 3D printing (after gelation in $1 \mathrm{M} \mathrm{NaOH}$ followed by $\mathrm{pH}$ neutralization and air-drying). Each of these stages was analysed via SEM and XRD.

Once an understanding of the effect of the presence of the biopolymer on the $\mathrm{CaCO}_{3}$ morphologies generated is obtained, the possibility of modifying and controlling the resultant structure of the crystallites can be explored. Ultimately, tailoring the formed morphology to, for example, the desired pancake-like structure through the addition of PAA as the crystal growth modifier to the blended hydrogel system.

On this basis, the morphology of the $\mathrm{CaCO}_{3}$ crystallites present after the blended hydrogel ink preparation step was investigated initially. Printing inks based on $\mathrm{Ch}-\mathrm{CaCO}_{3}$ gels with different $\mathrm{CaCO}_{3}$ percentages were prepared via the modified method (i.e. with varying concentrations of acetic acid added in order to improve the printability of the gels). $10 \mathrm{~mL}$ 
of each of the gels were cast in Petri dishes and air-dried to form thin films. These were then analysed via SEM and XRD.

Secondly, 3D Ch-CaCO 3 blended hydrogel-based scaffolds, $15 \mathrm{~mm}$ in diameter and $5 \mathrm{~mm}$ in height, based on the CAD model described in Chapter 2, were fabricated. The as-printed scaffolds were rested in $1 \mathrm{M} \mathrm{NaOH}$ and then washed with purified water until neutral $\mathrm{pH}$. To analyse the morphology of the resultant $\mathrm{CaCO}_{3}$ crystallites within the $3 \mathrm{D}$ composite via SEM, the 3D prints were delaminated, when in the wet state, to expose the underlying printed layers and then air-dried. XRD analysis was also performed.

Thirdly, to deduce the effect of the presence of the chitosan hydrogel on the resultant morphologies of calcium carbonate crystallites, the morphologies observed in the above two steps were correlated with those obtained under similar experimental conditions but in the absence of chitosan. Essentially, experiments were performed in the absence of chitosan while maintaining the reaction conditions as close as possible to those when using chitosan. Accordingly, the same proportions of the chemicals were reacted as if to develop the printing ink or to replicate the reaction during 3D printing, but in the absence of chitosan. For example, to make a fake ink mimicking blended inks with $10 \% \mathrm{CaCO}_{3}, 10 \% \mathrm{w} / \mathrm{w}$ $\mathrm{CaCO}_{3}$ (mass calculated w.r.t to $5 \%$ w/v chitosan, as calculated when preparing $\mathrm{Ch}-\mathrm{CaCO}_{3}$ gel with $10 \%$ w/w $\mathrm{CaCO}_{3}$ ) is reacted with $5 \%$ v/v acetic acid. Similarly, $50 \%$ w/w $\mathrm{CaCO}_{3}$ (mass calculated w.r.t to $5 \%$ w/v chitosan, as calculated when preparing $\mathrm{Ch}-\mathrm{CaCO}_{3}$ gel with $50 \%$ w/w $\mathrm{CaCO}_{3}$ ) was reacted with $10 \% \mathrm{v} / \mathrm{v}$ acetic acid. Likewise, to a make fake composite that mimics the final $\mathrm{Ch}-\mathrm{CaCO}_{3}$ blended hydrogel-based composite, $10 \%$ w/w $\mathrm{CaCO}_{3}$ (amount calculated w.r.t to $5 \% \mathrm{w} / \mathrm{v}$ chitosan as used to develop the ink with $10 \% \mathrm{w} / \mathrm{w}$ $\mathrm{CaCO}_{3}$ ) was, for example, reacted with $5 \% \mathrm{v} / \mathrm{v}$ acetic acid; $1 \mathrm{M} \mathrm{NaOH}$ (ethanolic) was then added (to replicate the lamination process wherein ethanolic $\mathrm{NaOH}(1.5 \%-1 \mathrm{M} \mathrm{NaOH})$ is used and the gelation process involving prolonged soaking in $1 \mathrm{M}$ after printing). The reaction products were air-dried and analysed via XRD and SEM.

It is important to note that the $3 \mathrm{D}$ printing process designed in this research involves continued addition and removal of the ethanolic $\mathrm{NaOH}$ solution after printing each layer of the $3 \mathrm{D}$ structure throughout the printing process and prolonged soaking of the printed scaffold in $1 \mathrm{M} \mathrm{NaOH}$ for gelation. This kind of periodic interaction with a base may also constantly vary the morphology of the resultant $\mathrm{CaCO}_{3}$ crystallites within each layer of the final 3D composite. The variation thus introduced, was not explored in detail here, rather 
the effect of the presence of a polymeric system on the morphology of the resultant $\mathrm{CaCO}_{3}$ crystallites in the final composite structure as a result of all of these processes was explored.

Figures 4.4 and 4.6 show the corresponding SEM images and Figures 4.5 and 4.7 show the representative XRD data for the various experimental protocols. The XRD data presented are for the samples containing the highest percentage of $\mathrm{CaCO}_{3}(50 \%)$ for clarity as the only noticeable differences observed in the XRD plots for the final 3D printed composites fabricated using different blended ink compositions, was the increasing prominence of the peaks corresponding to the crystalline plane $((022))$ in chitosan with decreasing $\mathrm{CaCO}_{3}$ content in the test sample. In the case of the blended inks, XRD plots did not reveal extensive variations in the crystallinity of the samples with increasing $\mathrm{CaCO}_{3}$ loading. Herein, blended hydrogels with $30 \% \mathrm{CaCO}_{3}$ was shown as representative samples, as the few crystalline peaks that were detected were more prominent in these.

\subsection{3a Blended hydrogel ink preparation}

When $\mathrm{CaCO}_{3}$ is reacted with acetic acid, a by-product is calcium acetate. The anhydrous form of calcium acetate is very hygroscopic and therefore it commonly exists as the monohydrate $\mathrm{Ca}\left(\mathrm{CH}_{3} \mathrm{COO}\right)_{2} \cdot \mathrm{H}_{2} \mathrm{O}$.

$$
\mathrm{CaCO}_{3}(\mathrm{~s})+2 \mathrm{CH}_{3} \mathrm{COOH}(\mathrm{aq}) \longrightarrow \mathrm{Ca}\left(\mathrm{CH}_{3} \mathrm{COO}\right)_{2}(\mathrm{~s})+\mathrm{H}_{2} \mathrm{O}+\mathrm{CO}_{2} \uparrow
$$

XRD data for the reaction product of calcium carbonate with acetic acid in the absence of chitosan indicated the presence of $\mathrm{Ca}\left(\mathrm{CH}_{3} \mathrm{COO}\right)_{2} \cdot \mathrm{H}_{2} \mathrm{O}$ conforming predominantly to an orthorhombic unit cell. Peaks corresponding to its half hydrated form $\left.\mathrm{Ca}\left(\mathrm{CH}_{3} \mathrm{COO}\right)_{2} \cdot 0.5 \mathrm{H}_{2} \mathrm{O}\right)[16]$ and to calcium carbonate (calcite, rhombohedral until cell) are also observed. SEM images (Figure 4.4 (a-e)) reveal that the resultant crystallites predominantly exist as aggregates of thin needle-like structures irrespective of the concentration of the reactants used.

When the same reaction was carried out in the presence of chitosan, the reaction products and the morphology of the resultant crystallites formed within the hydrogel were different than those obtained in the absence of chitosan. XRD analysis of the blended hydrogel (Ch$\mathrm{CaCO}_{3}$ ) films did not show as many crystalline planes, suggesting the presence of chitosan dominating the data. However, some low-intensity peaks corresponding to the crystalline planes of calcium acetate hydrate and calcium carbonate (calcite) could be identified. SEM analysis showed that the morphology of the resultant crystallites were random aggregates 
of micron-sized crystallites, entirely different from the needle-like aggregates obtained when the reaction was performed in the absence of chitosan.

According to SEM images, at a constant $\mathrm{pH}$ and polymer content (within the polymermineral system), complete dissolution of the added $\mathrm{CaCO}_{3}$ was observed only when the $\mathrm{CaCO}_{3}$ percentage loading was low, despite there being no suspended material readily visible to the naked eye in any of the prepared gels. The SEM image for hydrogels with $10 \%$ w/w $\mathrm{CaCO}_{3}$ (Figure 4.4 (f)) indicates that the added $\mathrm{CaCO}_{3}$ has dissolved completely, yielding a uniform blended hydrogel. As the percentage of the added $\mathrm{CaCO}_{3}$ increased at a fixed $\mathrm{pH}$, the size, the number of particles and the extent of aggregation observed within the resultant blended hydrogel also increased. The same trend was observed in blended inks prepared at a lower $\mathrm{pH}$ (i.e. when using $10 \% \mathrm{v} / \mathrm{v}$ acetic acid). The SEM images of blended inks with $40 \%$ w/w $\mathrm{CaCO}_{3}$ (Figure 4.4 (i)) reveal that complete dissolution of the added $\mathrm{CaCO}_{3}$ was not obtained in this case, rather nano to micron-sized particles were visible within the polymeric system. The data indicates that blended inks with $20 \% \mathrm{w} / \mathrm{w} \mathrm{CaCO}_{3}$ (in the presence of $5 \% \mathrm{v} / \mathrm{v}$ acetic acid) is directly comparable with those prepared using $40 \%$ w/w $\mathrm{CaCO}_{3}$ (in the presence of $10 \%$ v/v acetic acid) and similarly blended inks with $30 \%$ w/w $\mathrm{CaCO}_{3}$ (in the presence of $5 \%$ v/v acetic acid) to those with $50 \% \mathrm{w} / \mathrm{w} \mathrm{CaCO}_{3}$ (in the presence of $10 \% \mathrm{v} / \mathrm{v}$ acetic acid).

Based on the SEM data obtained, it can be suggested that $\mathrm{CaCO}_{3}$ crystallites undergo a dissolution-precipitation cycle during the preparation of the printing ink, this process being more prominent as the $\mathrm{CaCO}_{3}$ loading percentage is increased while keeping the $\mathrm{pH}$ of the system constant. This suggests that the resultant mineral phase within the blended ink will undergo a constant variation in the morphology and the size of the crystallites during the blending process.

Additionally, the difference in the composition and morphology of the crystallites present in the blended inks from those obtained when chitosan is absent suggests that the presence of the polymer restricts the extent of interaction between the calcium carbonate particles with the acid. The data also indicates that such restricted interactions result in inks with different mineral species being present rather than just pure calcium carbonate or calcium acetate. Consequently, as there are now different calcium ion sources, these will respond differently during any further processing of the blended hydrogel or to the inclusion of any additive such as PAA compared with the system described in Chapter 3. Hence, in seeking to simplify the system, several elements have introduced more complexity into the system. 


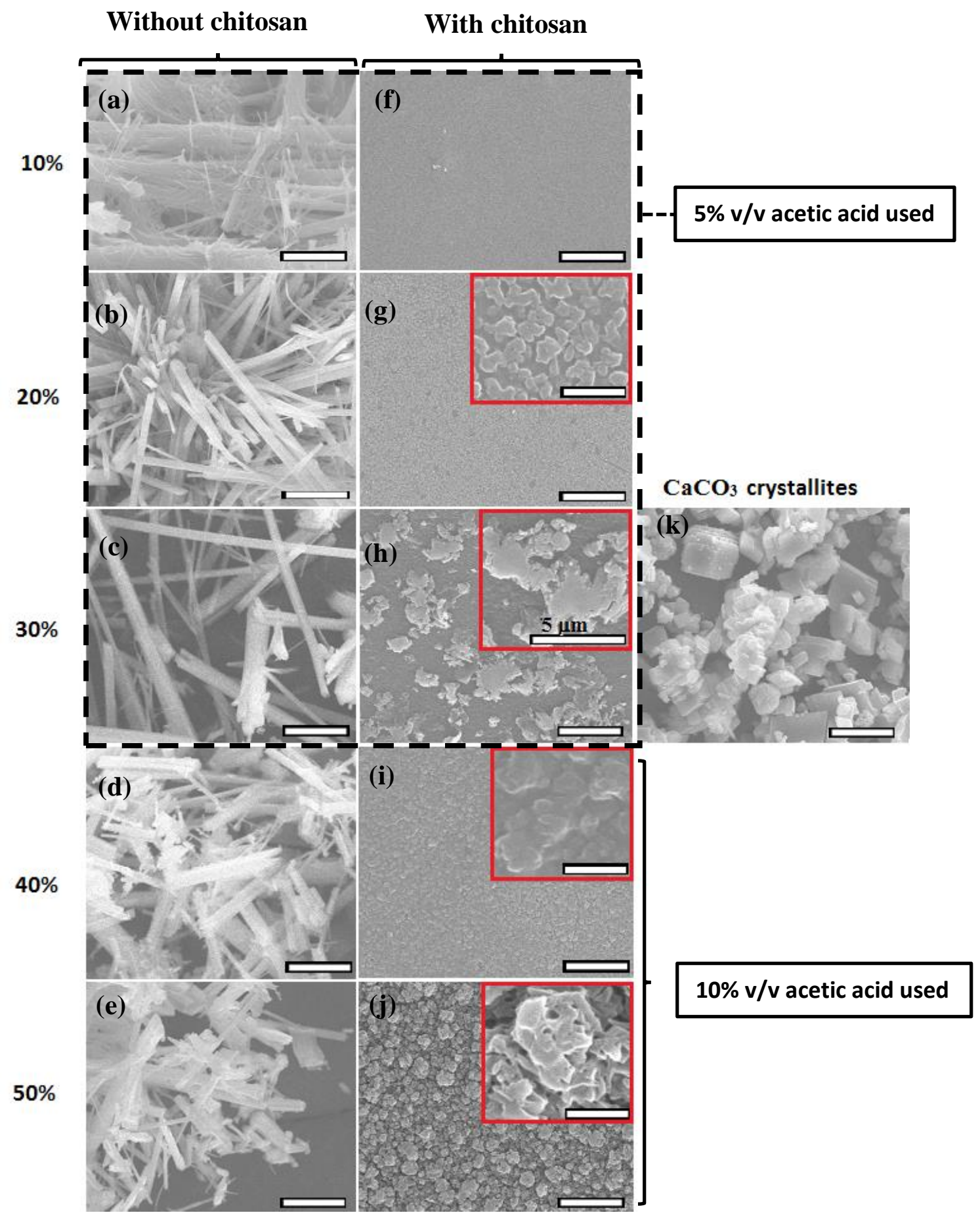

Figure 4.4: SEM images of crystallites obtained under different reaction conditions: (a-e) $10 \%-50 \%$ calcium carbonate reacted with varying concentrations of acetic acid (using the same concentration for all reagents as those used to prepare the Ch-CaCO 3 gel-based printing inks with $5 \% \mathrm{w} / \mathrm{v}$ chitosan but, in the absence of chitosan); and (f-j) $\mathrm{Ch}-\mathrm{CaCO}_{3}$ hydrogels (used as the printing ink) with varying concentration of added $\mathrm{CaCO}_{3}$ (10\%50\%). Also shown is $(k)$ the morphology of $\mathrm{CaCO}_{3}$ crystallites as supplied by the manufacturer. Scale for images is $10 \mu \mathrm{m}$ and those of the inset images is 1 um unless otherwise mentioned. 


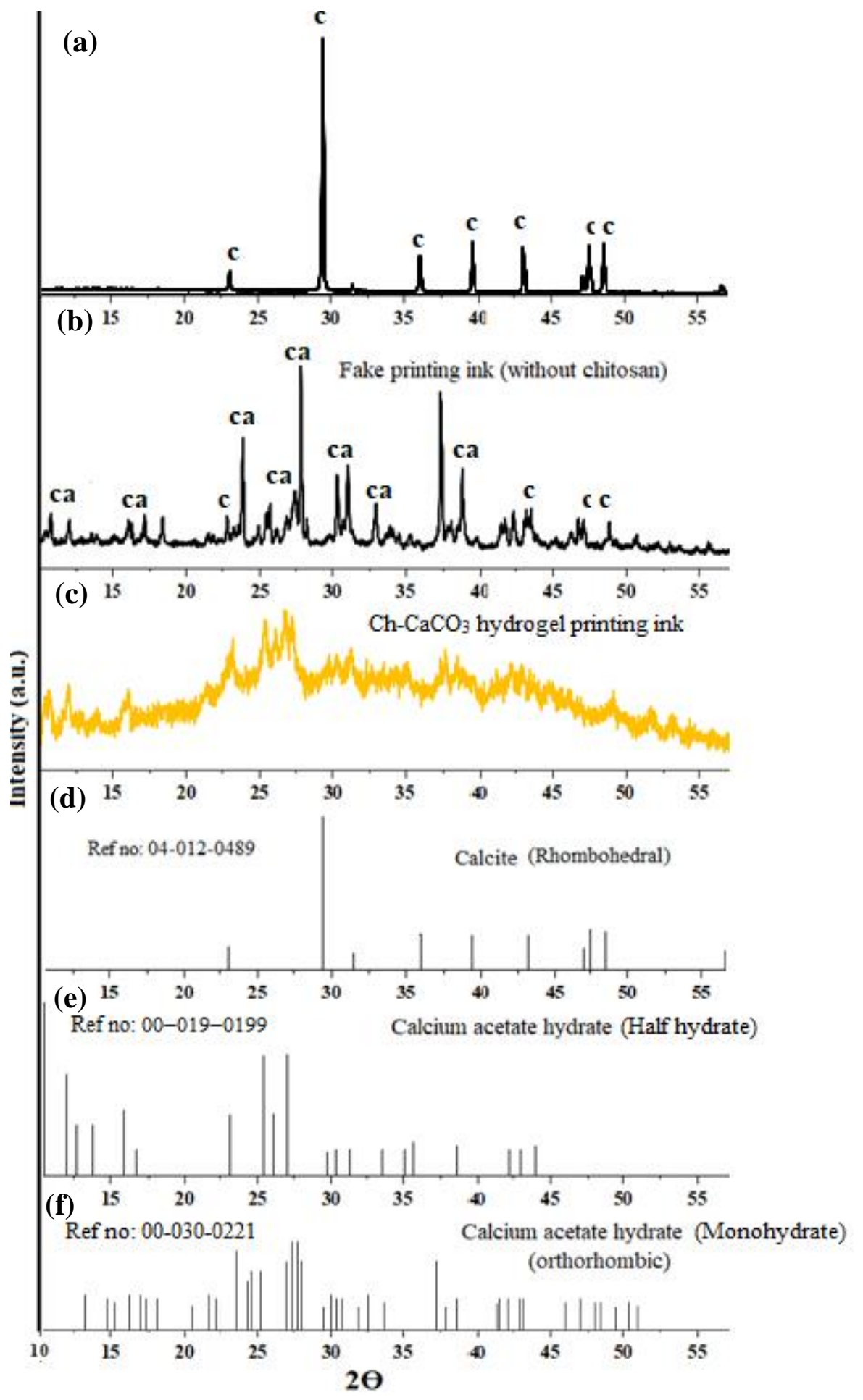

Figure 4.5: Representative XRD data for (a) calcium carbonate as obtained from the manufacturer; (b) the reaction product of $30 \% \mathrm{w} / \mathrm{w} \mathrm{CaCO}_{3}$ (w.r.t $5 \% \mathrm{w} / \mathrm{v}$ chitosan) $+5 \%$ acetic acid (AA) i.e. the fake printing ink without chitosan; and (c) $\mathrm{Ch}-\mathrm{CaCO}_{3}$ hydrogelbased printing ink with $50 \% \mathrm{w} / \mathrm{w} \mathrm{CaCO}_{3}$ (wr.t $5 \% \mathrm{w} / \mathrm{w}$ chitosan). Also shown are reference peaks for $(d)$ calcium carbonate (rhombohedral calcite) (peaks labelled 'c' in (a) and (b)); and $(e, f)$ calcium acetate hydrate of different hydration content (peaks labelled ' $c a$ ') (X'Pert Highscore 2.2c). 


\subsection{3b 3D printing}

XRD data showed that when the reaction product of calcium carbonate and acetic acid was then reacted with $1 \mathrm{M} \mathrm{NaOH}$ (ethanolic) (fake composites), mimicking the treatment used in the $3 \mathrm{D}$ printing process but in the absence of chitosan the final product predominantly contained calcite. However, a few high intensity peaks indicating the presence of monohydrate (orthorhombic unit cell) and half hydrate of calcium acetate are also observed.

In contrast, the XRD analysis of 3D composites fabricated using blended inks (after gelation in $1 \mathrm{M} \mathrm{NaOH}, \mathrm{pH}$ neutralization and air-drying) reveals that the mineral phase was essentially rhombohedral calcite irrespective of the concentration of $\mathrm{CaCO}_{3}$ initially added during the preparation of the blended inks.

SEM images (Figure 4.6) reveal that the morphology of the resultant crystallites was different when chitosan was (Figure 4.6 (a-e)) or was not present (Figure 4.6 (f-j)). In the case of the latter, the resultant crystallites were predominantly in the form of aggregates of thin needle-like structures irrespective of the initial concentration of $\mathrm{CaCO}_{3}$ added to the printing ink or $\mathrm{pH}$ of the system, which is different from the random structures obtained in the case of the former. This is similar to the trend observed in blended inks with varying $\mathrm{CaCO}_{3}$ concentration and the corresponding fake inks fabricated under same reaction conditions but in the absence of chitosan. Hence, confirming the previous hypothesis that the blending of chitosan polymer chains with inorganic phase restricts the interaction of the reactants involved with the inorganics, hence resulting in morphologies different from when the reactions are performed in the absence of chitosan.

The SEM data revealed that the trend observed in terms of the morphology and size of the resultant crystallites with the variation in $\mathrm{pH}$ in blended inks (Figure 4.4 (f-j)) was mimicked in the final composites as well (Figure 4.6 (a-e)). SEM images of the final composites revealed that size of the resultant crystallites increased with the concentration of $\mathrm{CaCO}_{3}$ initially incorporated in the blended inks at a constant $\mathrm{pH}$. For example, in the case of composites fabricated using blended inks loaded with $10-30 \% \mathrm{w} / \mathrm{w} \mathrm{CaCO}_{3}$ in the presence of $5 \% \mathrm{v} / \mathrm{v}$ acetic acid, the size of the mineral particles within the final composite increased with the concentration of $\mathrm{CaCO}_{3}$ initially loaded in the printing ink. The same was true in the case of composites fabricated using blended inks loaded with $40-50 \%$ w/w $\mathrm{CaCO}_{3}$ in the presence of $10 \% \mathrm{v} / \mathrm{v}$ acetic acid. When using blended inks with high mineral loading 
(40-50\%), it was observed that the resultant crystallites in the final 3D printed composites were in the form of aggregates of nano-sized $\mathrm{CaCO}_{3}$ crystallites (Figure $4.6(\mathrm{~d}, \mathrm{e})$ ).

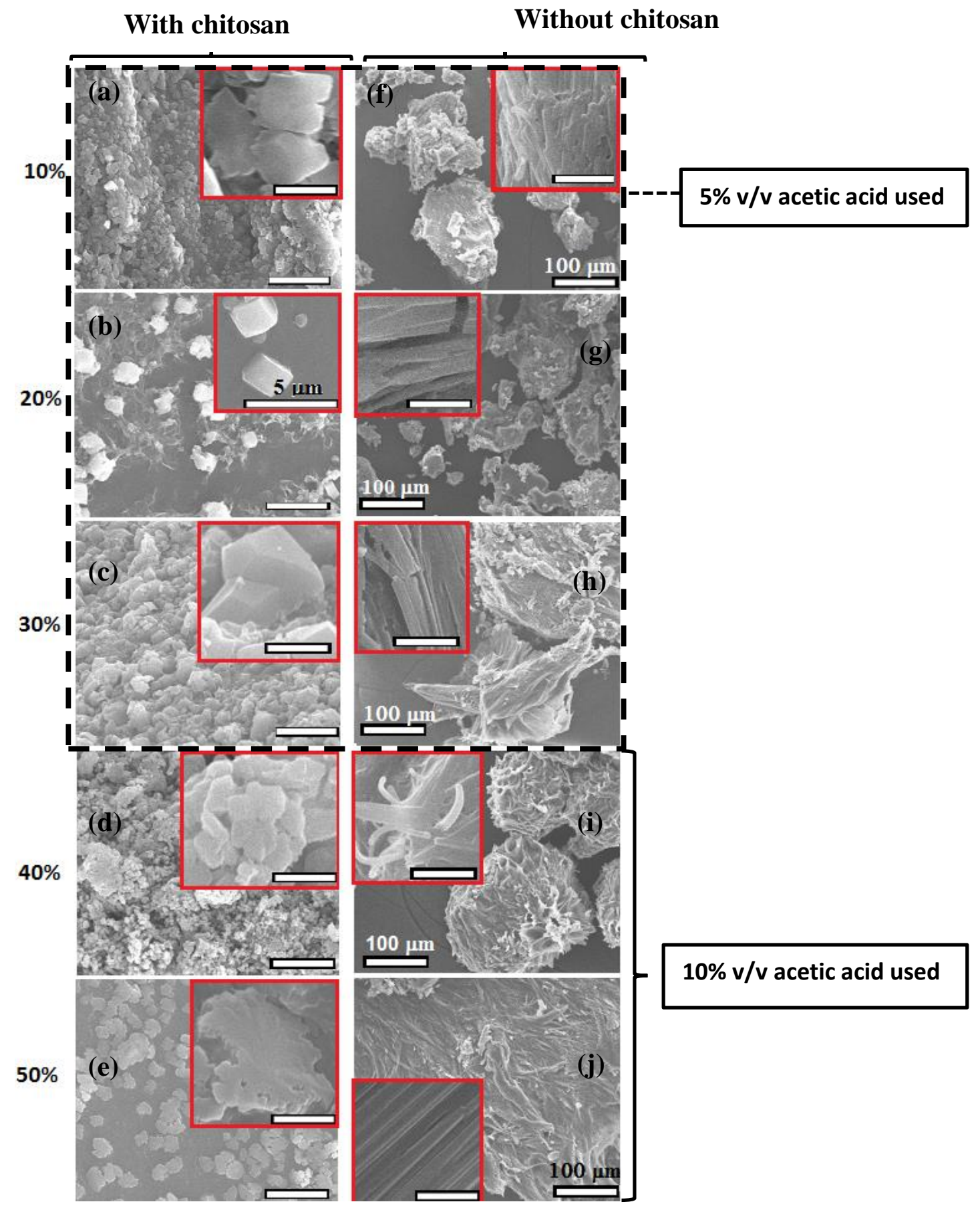

Figure 4.6 SEM images of crystallites obtained under different reaction conditions: (a-e) the final $3 \mathrm{D}$ printed composites fabricated using $\mathrm{Ch}-\mathrm{CaCO}_{3}$ blended inks with varying concentration of $\mathrm{CaCO}_{3}$ added (10\%-50\%) after gelation in $1 \mathrm{M} \mathrm{NaOH}$, pH neutralization and air-drying; and (f-j) 10\%-50\% calcium carbonate (w.r.t 5\% w/v chitosan) reacted with varying concentrations of acetic acid and $1 \mathrm{M}$ ethanolic NaOH. Scale for images is $10 \mu \mathrm{m}$ and of the inset images is 1 mm unless otherwise mentioned. 


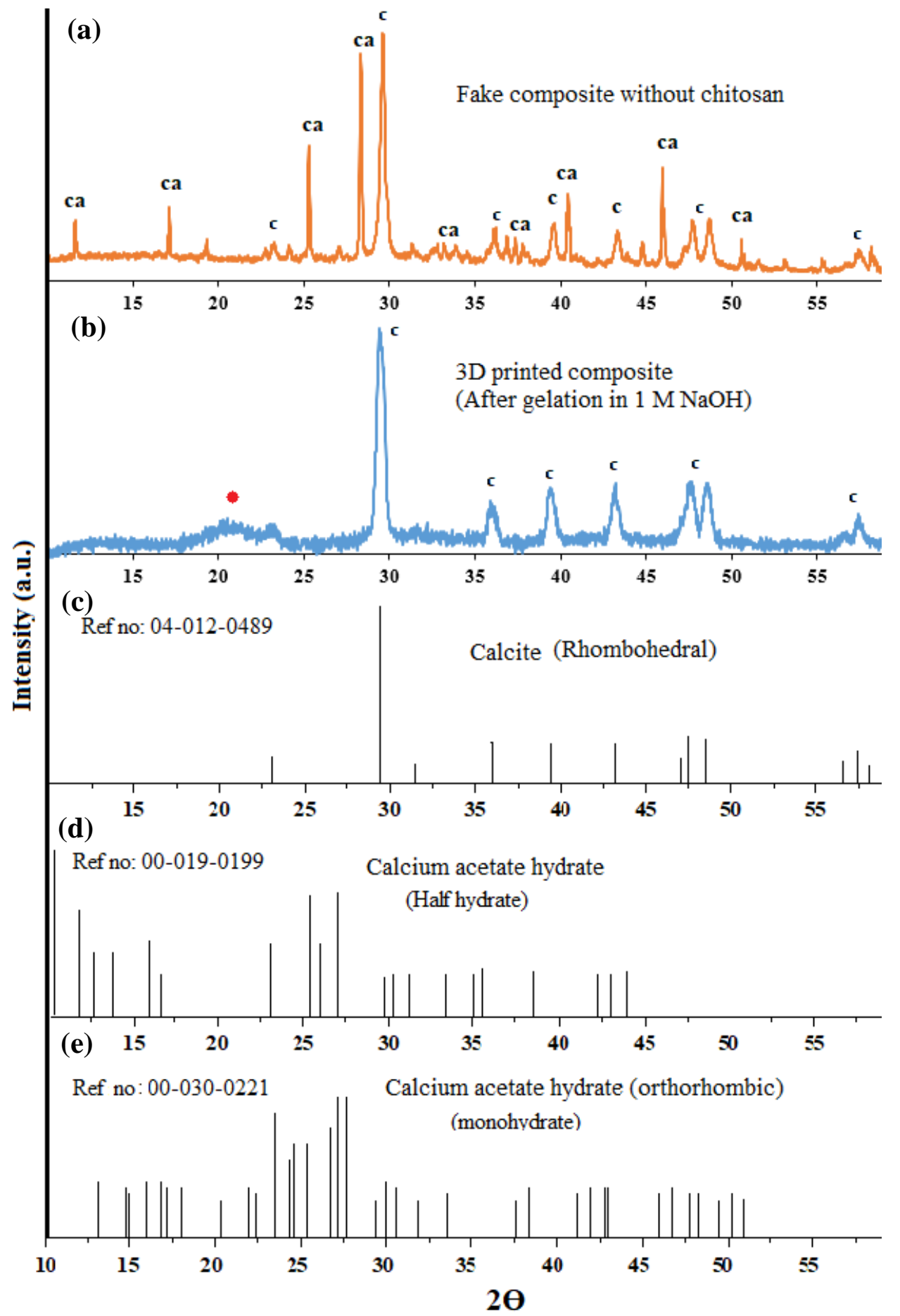

Figure 4.7: Representative XRD data (a) of the reaction products of $50 \% \mathrm{w} / \mathrm{w} \mathrm{CaCO}_{3}$ (w.r.t $5 \% \mathrm{w} / \mathrm{v}$ chitosan) $+10 \% \mathrm{v} / \mathrm{v}$ acetic acid $(\mathrm{AA})+1 \mathrm{MNaOH}$ (ethanolic) i.e. the fake composite without chitosan; and (b) the final $3 \mathrm{D}$ printed composite fabricated using $\mathrm{Ch}-\mathrm{CaCO}$ blended hydrogel loaded with $50 \% \mathrm{w} / \mathrm{w} \mathrm{CaCO}_{3}$. Also shown are reference peaks for $(c)$ calcium carbonate (calcite) (peaks labelled ' $c$ ' in (a) and (b)); and ( $d$, e) calcium acetate hydrate with different hydration content (peaks labelled ' $c a$ ' in (a)) (X'Pert Highscore 2.2c). The broad peak marked by a red dot in (b) corresponds to chitosan. 
Furthermore, SEM images indicate that, in composites fabricated using the single-step process (using blended inks), the $\mathrm{CaCO}_{3}$ crystallites formed within the composite were not associated with the polymer matrix (nature of this interaction is explained further in next section). This is similar to the calcium carbonate crystal growth observed in composites fabricated using the two-step fabrication technique, wherein 3D printed chitosan hydrogelbased scaffolds are mineralized via the McGrath method in the absence of PAA.

\subsubsection{Effect of introduction of crystal growth modifier (PAA)}

Once the composition and the morphology of the composite were accounted for, strategies to modify the resultant morphology of the calcium carbonate crystallites to form the desired pancake-like structure were investigated. PAA has been successfully used to serve this purpose in the two-step fabrication process involving the application of the McGrath method, hence the effect of the same additive in the single-step fabrication process was investigated.

Unlike the two-step fabrication process, wherein PAA is added to the mineralization media in which the 3D printed chitosan hydrogel-based scaffold is soaked, in the single-step fabrication process, PAA is incorporated directly into the blended printing ink during the gel preparation step. Accordingly, hydrogels were prepared with $2.5 \%$ w/w PAA (w.r.t to $5 \% \mathrm{w} / \mathrm{v}$ chitosan), incorporated via mechanical stirring during the hydrogel ink preparation step (based on the modified protocol described in Section 4.2) and then used as the printing ink to develop 3D structures.

Scaffolds fabricated via the two techniques (in the presence or absence of PAA) appear physically similar to each other in the wet state.

Figure 4.8 shows a comparison between representative optical and SEM images of chitosan$\mathrm{CaCO}_{3}$ composites fabricated using $\mathrm{Ch}-\mathrm{CaCO}_{3}$ blended hydrogels $\left(30 \% \mathrm{CaCO}_{3}\right.$ loading $)$ with $2.5 \%$ w/w PAA (w.r.t $5 \%$ w/v chitosan) added $\left(\mathrm{Ch}-\mathrm{CaCO}_{3}-\mathrm{PAA}\right)$ and chitosan- $\mathrm{CaCO}_{3}$ composites fabricated by mineralization of chitosan hydrogel-based scaffolds via the McGrath method (alternate soaking in $0.1 \mathrm{M}$ precursor ion solutions (24 h each) + soaking in Kitano ( 1 week) in the presence of $2.5 \%$ w/w PAA (w.r.t to dry weight of chitosan used). The morphology of crystallites observed in the regions directly exposed to the mineralization media and those formed in between the layers are shown in the case of the latter. TGA analysis (TGA of composites fabricated using the blended inks are discussed in Section 4.3.4, refer to Chapter 3, Table 3.4 for the TGA analysis of composites developed 
via the McGrath mineralization method) showed that the final mineral content in the composites fabricated via the two fabrication techniques (based on the protocol described above in each case) is comparable.

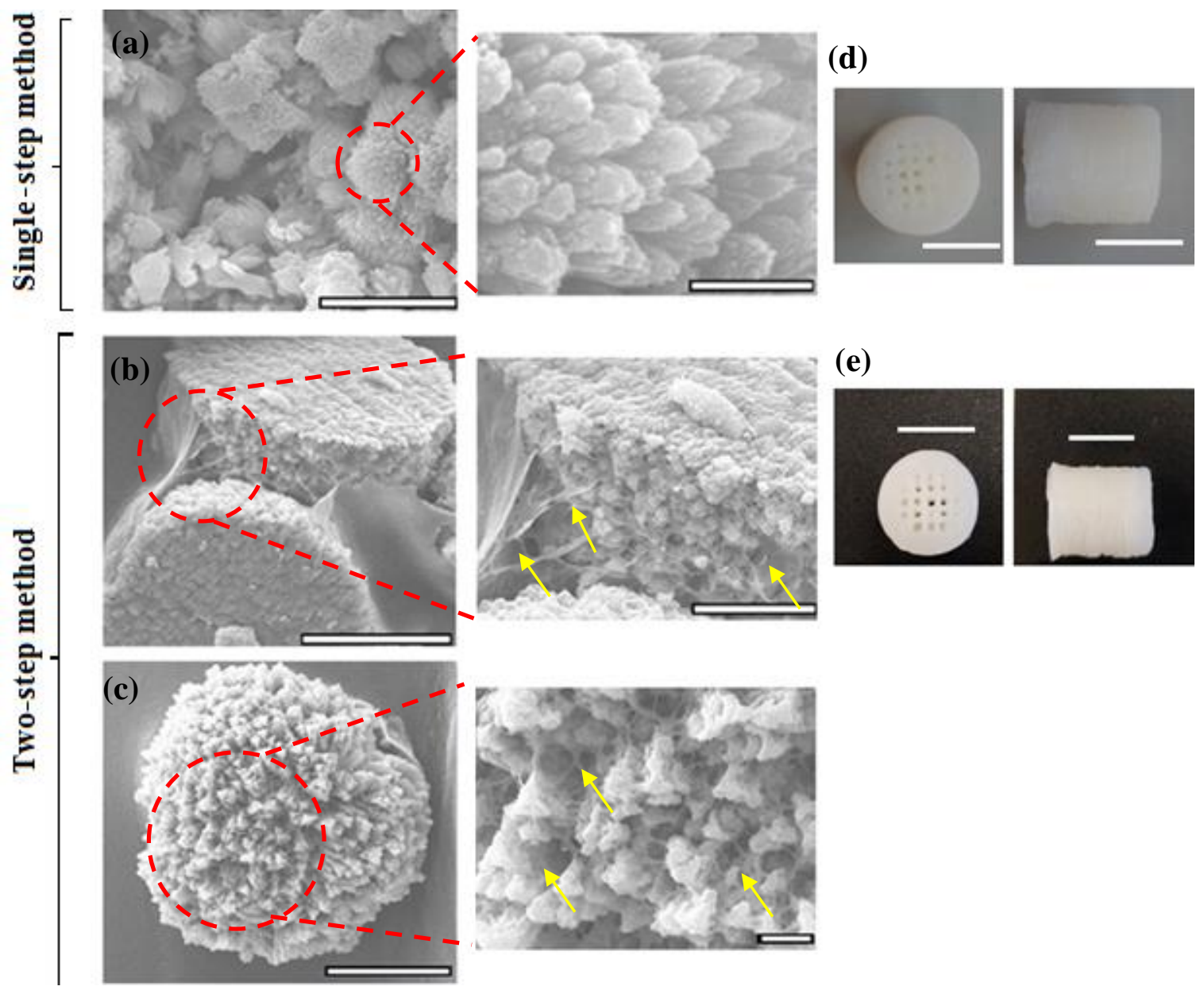

Figure 4.8: A comparison of the $\mathrm{CaCO}_{3}$ morphologies formed in the $3 \mathrm{D}$ printed chitosan$\mathrm{CaCO}_{3}$ composites fabricated via $3 \mathrm{D}$ printing using (a) $\mathrm{Ch}-\mathrm{CaCO}_{3}$ blended inks loaded with $30 \% \mathrm{w} / \mathrm{w} \mathrm{CaCO}_{3}$ and $2.5 \%$ w/w PAA (both w.r.t $5 \%$ w/v chitosan; mechanically blended together during ink preparation); and (b-c) chitosan hydrogel as the printing ink followed by mineralization of the as-fabricated chitosan hydrogel-based scaffold via the McGrath mineralization method in the presence of $2.5 \% \mathrm{w} / \mathrm{w}$ PAA (w.r.t the dry weight of chitosan used), crystallite morphologies observed in the regions directly exposed to the mineralization media $(b)$ and in between the printed layers $(c)$. On the right-hand side are higher resolution images of the same. $(d, e)$ are the corresponding optical images of the final composites based on (a) and (b) respectively. Scale bar, left-hand side images, $10 \mu \mathrm{m}$, high resolution images $1 \mu \mathrm{m}$ and optical images $1 \mathrm{~cm}$. The yellow arrows show the organic matrix, as differentiated from the calcium carbonate, which is intimately associated with the crystallites. This was not observed in the composite fabricated by the single-step method. 
Introducing PAA into the McGrath mineralization process, when using low concentration precursor ion solution, resulted in the formation of laterally growing pancake-like structures in the regions of the chitosan hydrogel-based scaffold that were directly exposed to the mineralization media. As explained in Chapter 3, high magnification images revealed that these pancake-like structures are in fact formed of nanoparticles that are associated with the hydrogel matrix. Similarly, in between the printed layers, smaller laterally growing aggregates of nanoparticles are observed wherein the nanoparticles are clearly fully intermeshed within the hydrogel matrix (Figure 4.8 (c)). The interactions between the nanoparticles and the hydrogel matrix described here are non-covalent in nature and are the result of electrostatic interactions between the $\mathrm{COO}^{-}$groups in $\mathrm{PAA}$ and the $\mathrm{NH}_{4}{ }^{+}$groups in the chitosan polymer chain and the resultant formation of the polymer electrolyte complex as described in Chapter 3. Polyelectrolyte formation does not occur in the absence of PAA, thus the observed lack of interaction between the crystallites and matrix as mentioned in the previous section.

Introduction of PAA in the $\mathrm{Ch}-\mathrm{CaCO}_{3}$ blended gel modified the morphology of the resultant crystallites from those obtained in the absence of PAA. SEM images showed that the majority of the resultant crystallites within the final $3 \mathrm{D}$ printed $\mathrm{Ch}-\mathrm{CaCO}_{3}-\mathrm{PAA}$ composites exist as aggregates of calcium carbonate needles with a preferential outward orientation (Figure 4.8 (a)) in comparison to the random aggregates obtained in the absence of PAA (Figure 4.6 (a-e)). However, the SEM images did not reveal any distinguishable association of the crystallites with the hydrogel matrix in comparison to the defined association observed (polymer-mineral entanglements, depicted by the yellow arrows in Figure 4.8) in the case of composites fabricated via the two-step process involving the McGrath mineralization of chitosan hydrogel-based scaffolds.

In the two-step fabrication process, the polyelectrolyte complex formed between PAA and the chitosan polymer chains during the McGrath mineralization process ensures that the hydrogel provides low energy nucleation sites for calcium carbonate. Thus, the mineral growth occurs while in contact with the organic matrix. The presence of the entangled hydrogel network along with the nanoparticle aggregates (yellow arrows in Figure 4.8) is evidence of this association. It can also be suggested that the polyelectrolyte complex formation reduces the surface tension between the particles and the polymer ensuring uniform nucleation of calcium carbonate crystallites while it is still entangled with the polymer chain [17]. 
In the single-step fabrication of composites using $\mathrm{Ch}-\mathrm{CaCO}_{3}-\mathrm{PAA}$ blended inks, it is proposed that the lack of polymer-matrix-mineral interactions during formation of the crystallites and the subsequent aggregation of these in to the final crystallites leads to there being no real entanglement with the hydrogel matrix. This could be because of the way in which the polyelectrolyte complex forms when using this fabrication technique (i.e., mixing of all the reactant species involved with PAA in a single-step reaction). It is possible that PAA-Ca ${ }^{2+}$ polyelectrolyte complex formation occurs instead of $\mathrm{PAA}^{-\mathrm{NH}_{4}}{ }^{+}$, which favours the initial crystallite nucleation within the polymer matrix. Thus, resulting in the growth of the crystallite without an enhanced interaction/association with the chitosan hydrogel chains $[15,18]$. This along with the various reaction parameters, such as the $\mathrm{pH}$, may have altered the nature of the interaction between the PAA and the chitosan matrix due to different numbers and distribution of various functional groups, thus affecting the crystallization kinetics of calcium carbonate.

Literature suggests that the strength of interactions between the mineral and the matrix within biopolymer-based composites directly affects their mechanical properties and that high mineral content may have detrimental effects on the mechanical strength (main research interest of this research) of the final composite unless the strength of the interaction between the matrix and filler are maintained or enhanced [19, 20]. Given the lack of evidence for a close physical association between the organic and inorganic components of the composites formed using the single-step method, it is concluded therefore that Ch$\mathrm{CaCO}_{3}$-PAA blended hydrogel-based composites may not have the desired superior mechanical properties compared with those fabricated by the two-step method due to the lack of good polymer-matrix interactions.

\subsubsection{Mineral leaching in 3D printed $\mathrm{Ch}-\mathrm{CaCO}_{3}$ composites}

During 3D printing using $\mathrm{Ch}-\mathrm{CaCO}_{3}$ blended hydrogels as the printing ink, it was observed that precipitation occurred during the lamination process. A white precipitate leaches out of the printed structure during lamination as the printed structure reacts with the ethanolic $\mathrm{NaOH}$. Although leaching may occur with an increased period of exposure, as in the case of all blended inks, the precipitation during the lamination process (done manually during the $20 \mathrm{~s}$ pause in the printing process) was visually more rapid and evident to the naked eye when using blended inks with higher calcium carbonate loading percentage (30 - 50\% $\mathrm{CaCO}_{3}$ loading). This phenomenon is likely to lead to a decrease in the final mineral content within the composites. To investigate this, the final 3D printed composites were thermally 
analysed via TGA. The resultant residual volume (\%) is compared with the expected calcium carbonate content (\%) of the final composites, determined by using the measured organic content as obtained via TGA analysis and the initial composition of the printing ink. Leaching was also observed when using $\mathrm{Ch}-\mathrm{CaCO}_{3}-\mathrm{PAA}$ blended hydrogels as the printing ink. Results are presented for the $\mathrm{Ch}-\mathrm{CaCO}_{3}$ composites only since it was observed that the presence of PAA did not have any effect on enhancing the polymer matrix interaction or the size of the resultant crystallite. It was therefore assumed that the observed leaching phenomena would be comparable for the composites with and without PAA added.

Accordingly, 3D scaffolds $15 \mathrm{~mm}$ in diameter $\times 4 \mathrm{~mm}$ in height were printed using blended inks with $10-50 \%$ w/w $\mathrm{CaCO}_{3}$ loading (w.r.t $5 \%$ w/v chitosan). The as-printed scaffolds were rested in $1 \mathrm{M} \mathrm{NaOH}$ to ensure complete gelation of the chitosan hydrogel and then washed with purified water until neutral $\mathrm{pH}$. The composite scaffolds were then air-dried and tested via TGA to determine the total mineral content. The data obtained are presented in Table 4.1. The thermal decomposition behaviour of composites based on 3D printed Ch$\mathrm{CaCO}_{3}$ blended inks (Figure 4.9) was the same as those fabricated via the two-step fabrication process involving the McGrath mineralization of 3D printed chitosan hydrogelbased scaffolds (Chapter 3, Section 3.3.6). The total mineral content of the sample is estimated based on the percentage residual volume remaining beyond the $4^{\text {th }}$ degradation step (i.e. beyond $600{ }^{\circ} \mathrm{C}$ ).

TGA data showed that the actual percentage mineral content of composite scaffolds fabricated using $\mathrm{Ch}-\mathrm{CaCO}_{3}$ blended hydrogels via the single-step fabrication process (based on the residual volume beyond $600{ }^{\circ} \mathrm{C}$ ), varied from the expected values. In some cases (composites generated using blended inks with $10 \%$ and $20 \%$ loading) the values obtained from TGA data are higher than the expected values and in other cases, it is lower (composites generated using blended inks with 30\% - 50\% loading) (Table 4.1).

Abert et al. explain that the tendency of filler particles to solve out of a composite is dependent on the size of the particles among other factors [21]. Reports also show that nanosized fillers increase the specific area of contact between the polymer and the mineral component which in turn affects the strength of the interactions between the mineral clusters and the organic matrix [22]. SEM images of the $\mathrm{Ch}-\mathrm{CaCO}_{3}$ blended inks with $10 \% \mathrm{w} / \mathrm{w}$ calcium carbonate loading (prepared in the presence of 5\% v/v acetic acid) reveal that the $\mathrm{CaCO}_{3}$ dissolved completely under the reaction conditions resulting in a uniform blended hydrogel (Figure 4.4 (f)). In the case of hydrogels with $20 \% \mathrm{CaCO}_{3}$ loading, the mineral 
loading was higher than the solubility limit of calcium carbonate under the reaction conditions and thus re-precipitated within the organic matrix in the form of nanostructures (Figure $4.4(\mathrm{~g})$ ). In the case of hydrogels with $30 \% \mathrm{CaCO}_{3}$ loading, the amount of mineral added was much higher than the solubility limit of calcium carbonate under the given $\mathrm{pH}$ conditions, hence, the resultant higher proportion of reprecipitation and formation of larger mineral crystallites within the organic matrix. This correlates to the observation for the blended inks loaded with $10 \%$ to $30 \% \mathrm{CaCO}_{3}$ prepared in the presence of $5 \% \mathrm{v} / \mathrm{v}$ acetic acid, whereby the white precipitate formed increased with increasing $\mathrm{CaCO}_{3}$ loading in the gel. The same is true in the case of blended inks with $40 \%$ to $50 \% \mathrm{CaCO}_{3}$ prepared in the presence of $10 \% \mathrm{v} / \mathrm{v}$ acetic acid. These data indicate that continuing to load more $\mathrm{CaCO}_{3}$ at a fixed $\mathrm{pH}$ results in limited solubility, leading to reprecipitation and hence weaker interaction with the organic scaffolds. Such weakly bound crystallites are more susceptible to being leached out of the organic matrix during the lamination process.

The varying degree of dissolution and re-precipitation of calcium carbonate occurring within the polymer matrix with respect to the initial calcium carbonate added and the $\mathrm{pH}$ of the system during the blending process and the lamination process may affect the thermal decomposition behaviour of the resultant blended inks and the final composites. In cases where the dissolution was complete, as in the gels loaded with $10 \% \mathrm{CaCO}_{3}$, very low amounts of mineral leaching was seen to occur during the lamination process and thus the final mineral content in the composite as obtained via TGA analysis should be comparable to the expected values calculated based on the initial calcium carbonate loading percentage. However, TGA data showed that the mineral content (based on the residual volume after thermal decomposition beyond $600^{\circ} \mathrm{C}$ ) was higher than the expected values. This suggests that the complete dissolution and then reprecipitation process that occurs during the ink preparation step; the subsequent lamination, gelation and drying processes may also have resulted in some of the chitosan being bound to or occluded in the calcium carbonate and thus being thermally shielded during decomposition. Hence, the measured residual volume will contain some undecomposed organic matrix along with the mineral components. This was also observed for composites fabricated via the two-step process as explained in Chapter 3. The TGA data also indicate that this may be the case for those composites fabricated using blended inks with $20 \% \mathrm{CaCO}_{3}$ loading despite the complete dissolution of the added $\mathrm{CaCO}_{3}$ not occurring during the blending process. 


\begin{tabular}{|c|c|c|}
\hline Type of composite scaffold & $\begin{array}{c}\text { Expected mineral } \\
\text { content in final } \\
\text { composite (based on the } \\
\text { initial gel composition) } \\
(\%)\end{array}$ & $\begin{array}{c}\text { Actual mineral } \\
\text { content in final } \\
\text { composite (based on } \\
\text { TGA) } \\
(\%)\end{array}$ \\
\hline $\begin{array}{c}\mathrm{Ch}-\mathrm{CaCO}_{3} \text { gel } \\
\left(10 \% \text { w/w } \mathrm{CaCO}_{3}+5 \% \text { AA }\right)\end{array}$ & $8.8 \pm 0.2$ & $12.3 \pm 1.6$ \\
\hline $\begin{array}{c}\mathrm{Ch}-\mathrm{CaCO}_{3} \text { gel } \\
\left(20 \% \text { w/w } \mathrm{CaCO}_{3}+5 \% \text { AA }\right)\end{array}$ & $16.0 \pm 0.1$ & $20.2 \pm 0.7$ \\
\hline $\begin{array}{c}\mathrm{Ch}-\mathrm{CaCO}_{3} \text { gel } \\
\left(30 \% \text { w/w } \mathrm{CaCO}_{3}+5 \% \text { AA }\right)\end{array}$ & $24.8 \pm 0.2$ & $17.4 \pm 0.8$ \\
\hline $\begin{array}{c}\mathrm{Ch}-\mathrm{CaCO}_{3} \text { gel } \\
\left(40 \% \text { w/w } \mathrm{CaCO}_{3}+10 \% \text { AA }\right)\end{array}$ & $28.9 \pm 0.8$ & $27.0 \pm 1.9$ \\
\hline $\begin{array}{c}\mathrm{Ch}-\mathrm{CaCO}_{3} \text { gel } \\
\left(50 \% \text { w/w } \mathrm{CaCO}_{3}+10 \% \text { AA }\right)\end{array}$ & $34.8 \pm 0.9$ & $30.3 \pm 1.8$ \\
\hline
\end{tabular}

Table 4.1: A comparison between the actual and expected minerals content within the final $3 \mathrm{D}$ printed composite $\left(15 \mathrm{~mm}\right.$ diameter $\times 4 \mathrm{~mm}$ high) fabricated using $\mathrm{Ch}-\mathrm{CaCO}_{3}$ blended inks with different calcium carbonate loading percentage.

Addition of fillers has been shown to improve the thermal stability of composites [23]. The phenomenon is observed in the case of blended ink-based composites developed via the single-step method as well. The thermal stability can be explained in terms of (a) the shift in the temperature at which the decomposition of a material begins; (b) the temperature at which various steps in the degradation process occur; and (c) the final residual volume after complete decomposition. Accordingly, by comparing the TGA plots of the blended inks and the respective final 3D printed composites (after gelation and air-drying) (Figure 4.9) the following were deduced:

(1) the fabrication process may have improved the thermal stability of the final composite, in terms of the temperature at which the $2^{\text {nd }}$ degradation step begins. In the case of the blended inks, there is seemingly continued weight loss beyond the initial loss of moisture content ( $1^{\text {st }}$ step in decomposition) unlike the constant weight loss observed before the $2^{\text {nd }}$ degradation step in the case of the final composites; 
(2) in the case of the final composites, the $4^{\text {th }}$ degradation step (beyond $600{ }^{\circ} \mathrm{C}$ ) marking the decomposition of calcium carbonate to $\mathrm{CaO}$, is not as prominent with the increased addition of calcium carbonate as in the case of the $4^{\text {th }}$ degradation step for corresponding blended inks. This suggests a loss of calcium carbonate during the 3D printing; and

(3) the continued addition of calcium carbonate to the blended ink (at constant $\mathrm{pH}$ ) did not affect the thermal decomposition behaviour of the resultant composites (i.e. no shift was observed in the temperature at which each of the degradation steps occurred). However, a variation in the percentage residual volume (beyond $600{ }^{\circ} \mathrm{C}$ ) is observed with the increase in initial calcium carbonate loading in the blended ink at constant pH. (Figure 4.9).

It should be noted that similar to the final composites, the residual volume (beyond $600{ }^{\circ} \mathrm{C}$ ) in the blended inks (data not shown in Table 4.1) is also affected by the initial $\mathrm{CaCO}_{3}$ loading percentage in the ink and the presence of undecomposed organic matter after complete thermal decomposition due to enhanced particle-polymer interactions as described earlier.
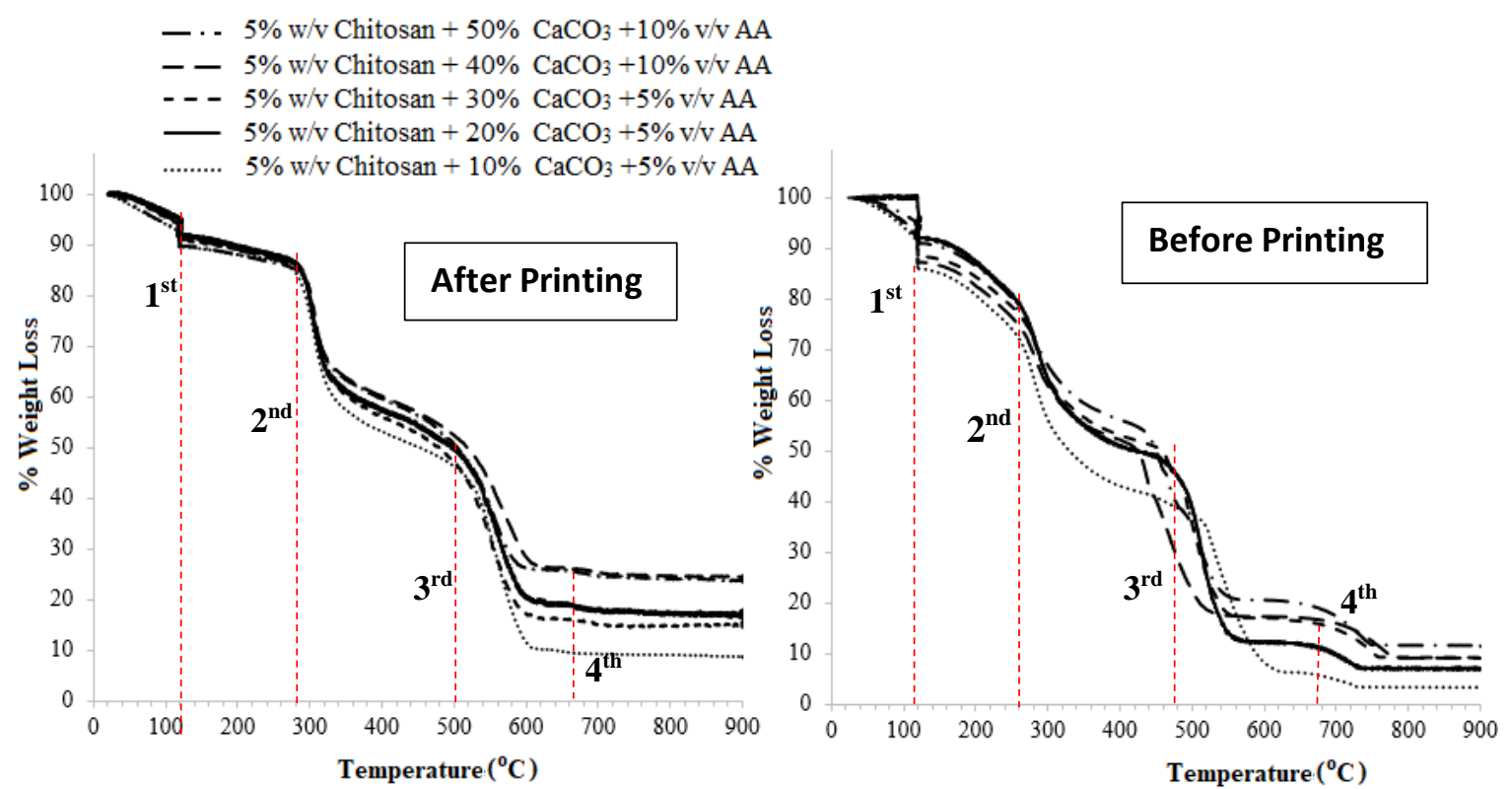

Figure 4.9: Thermal decomposition behaviour of blended inks with varying concentration of calcium carbonate added (on the right-hand side) and the corresponding $3 D$ printed composites (left-hand side). Red dotted lines represent the onset temperatures marking the decomposition of various components of the respective materials. 
Based on the TGA data and the observed loss in minerals during the lamination process (depicted by actual mineral content in Table 4.1 and the residual volume as in Figure 4.9, left-hand side image), it can be postulated that the comparatively lower residual volume (than expected) after thermal decomposition of the final composites fabricated using blended inks with high calcium carbonate loading (i.e., $30 \%$ or $50 \% \mathrm{CaCO}_{3}$ loading at the respective $\mathrm{pH}$ conditions) is a combined effect of the higher leaching observed during the lamination process and the possible increase in the residual volume due to undecomposed organic matrix after complete thermal decomposition of the final 3D composite. The actual mineral content being lower than the expected value suggests (Table 4.1) that the loss of minerals via leaching during lamination dominates over the possible increase in TGA residual volume due to undecomposed organic matter.

TGA data (Table 4.1) indicate that despite the observed precipitation during the lamination process, the best blended ink that resulted in composites with good thermal stability is the $\mathrm{Ch}-\mathrm{CaCO}_{3}$ blended ink with $40 \% \mathrm{CaCO}_{3}$ loading prepared in the presence of $10 \% \mathrm{v} / \mathrm{v}$ acetic acid. This can be correlated to the SEM image shown in Figure 4.4 (i), suggesting that a higher solubility and resultant enhanced mineral-matrix interaction may have led to the lower leaching of minerals during the lamination process. Furthermore, it is concluded that high mineral loading in blended inks (for a given $\mathrm{pH}$, for example, inks containing $30 \%$ $\mathrm{CaCO}_{3}$ or $50 \% \mathrm{CaCO}_{3}$ ) can result in proportionally higher leaching tendency, thus indicating the lack of efficiency of the fabrication in generating composites with preferred mineral content and thermal stability.

To further elucidate the disadvantages of the leaching phenomenon, another example is described. TGA analysis of a $19.2 \mathrm{mg}, 15 \mathrm{~mm}$ diameter $\times 8 \mathrm{~mm}$ high $3 \mathrm{D}$ printed composite fabricated using blended inks with $50 \%$ w/w $\mathrm{CaCO}_{3}$ loading (data not shown) revealed that of the total composite contained approximately $14.5 \mathrm{mg}$ of organic matter. Ideally, based on the initial gel composition, $50 \% \mathrm{w} / \mathrm{w}$ of the organic matrix should have been calcium carbonate i.e. $50 \%$ of $14.5 \mathrm{mg}$, equals $7.2 \mathrm{mg}$. However, TGA data showed that the total inorganic content (based on the residual weight remaining after thermal decomposition beyond $\left.600{ }^{\circ} \mathrm{C}\right)$ in the final composite was only about $4.6 \mathrm{mg}\left(24 \% \mathrm{w} / \mathrm{w} \mathrm{CaCO}_{3}\right)$. Comparing this data to the total mineral content within dimensionally smaller composite structures i.e., a $15 \mathrm{~mm}$ diameter $\times 4 \mathrm{~mm}$ high scaffold (as shown in Table 4.1), that are based on the same CAD model and fabricated using blended inks loaded with $50 \% \mathrm{w} / \mathrm{w}$ $\mathrm{CaCO}_{3}$, reveals that the total mineral content $(\%)$ within taller samples are much lower than 
that of the latter $\left(\sim 35 \% \mathrm{w} / \mathrm{w} \mathrm{CaCO}_{3}\right)$. Hence, it can be deduced that the percentage mineral leaching occurring during the lamination process increases with the volume (dimensions) of the printed structure, thus, continually reducing the final mineral content within the printed composite. This makes the single-step fabrication process less efficient in terms of developing composites with a prescribed mineral content.

It is noted that the two-step fabrication process has the following disadvantages: (1) the nonuniform distribution of crystallites throughout the 3D structure; (2) a lack of comprehensive control over the size and morphology of the calcium carbonate crystallites within the final composites; and (3) inability to restrict the nucleation of calcium carbonate to occur only within the scaffold and not on other surfaces. These issues, however, are not as unacceptable in comparison to the disadvantages of the single-step fabrication process with respect to (1) loss of mineral content during fabrication; (2) limitations in tuning the morphology of the resultant crystallites to form pancake-like structures; and (3) lack of extensive interaction between the organic and the inorganic components, all of which are key functionalities sought after in this research. Hence, based on the results from the investigations conducted, the application of this seemingly simpler fabrication technique in developing 3D chitosan$\mathrm{CaCO}_{3}$ composites with tuneable properties using hydrogels preloaded with $\mathrm{CaCO}_{3}$ was deemed unsuccessful in comparison to the two-step fabrication process developed in this research and presented in Chapter 3. 


\section{4: Conclusion}

The applicability of a simpler fabrication technique to develop 3D printed chitosan- $\mathrm{CaCO}_{3}$ composites using chitosan hydrogels preloaded with calcium carbonate crystallites over the two-step fabrication process described in Chapter 3, involving fabrication of 3D chitosan hydrogel-based scaffolds and mineralization using the McGrath method, was investigated.

Chitosan hydrogels with varying concentration of $\mathrm{CaCO}_{3}$ crystallites were prepared via mechanical blending and their rheological properties were studied. The viscosity of chitosan hydrogels was found to increase with the amount of calcium carbonate added, which in turn varied their flow properties from that of the ideal hydrogel suitable for printing in the custom-designed 3D printer. At higher strain rates i.e. at a range close to the strain rate experienced at the tip of a $0.42 \mathrm{~mm}$ extrusion nozzle during the printing process, the viscosity of the resultant blended hydrogels was comparable to that of the chitosan hydrogel ( $5 \% \mathrm{w} / \mathrm{v}$ chitosan dissolved in $2 \% \mathrm{v} / \mathrm{v}$ acetic acid) found to be a suitable printing ink in our 3D printer when using a $0.42 \mathrm{~mm}$ extrusion nozzle. However, at low strain rates, e.g. $0.1 \mathrm{~s}^{-}$ ${ }^{1}$ the former was $\sim 13$ to $\sim 100$ times higher than the latter depending on the $\mathrm{CaCO}_{3}$ loading, hence requiring a much higher pressure than the normal applied pressure, to initiate flow of the blended hydrogel through a $0.42 \mathrm{~mm}$ extrusion nozzle. Accordingly, the viscosity and the flow properties of the blended hydrogels were modified to match that of $5 \% \mathrm{w} / \mathrm{v}$ chitosan hydrogels (in 2\% v/v acetic acid) by increasing the concentration of acetic acid (increased from $5 \% \mathrm{w} / \mathrm{v}$ up to $10 \% \mathrm{w} / \mathrm{v}$ ) added during ink preparation.

Using the modified ink, 3D chitosan- $\mathrm{CaCO}_{3}$ scaffolds were successfully fabricated with 1.5 $\mathrm{mm} \times 1.5 \mathrm{~mm}$ macropores. The printed scaffolds were structurally stable at room temperature and pressure conditions and appeared similar to those fabricated via McGrath mineralization of chitosan hydrogel-based scaffolds (in the presence or absence of PAA) to physical observation.

In the absence of crystal growth modifiers such as PAA, the observed crystal growth was similar to that of the composites fabricated by the mineralization of chitosan hydrogel-based scaffolds via the McGrath method in the absence of PAA. As the single-step fabrication process involves the mechanical blending of the polymer and mineral crystallites, various steps such as the gel preparation and 3D printing process, were analysed to deduce the effect of the presence of the chitosan polymer on the morphology of the resultant calcium carbonate crystallites in the final material. It was found that the presence of chitosan 
polymer chains restricts the interaction of the acid/alkali with $\mathrm{CaCO}_{3}$ crystallites and thus the resultant crystallites assume a morphology different from those formed in the absence of chitosan. Also, this reduced and restricted interaction leads to a difference in the number and distribution of charged species for the organic matrix and the minerals for each step of the fabrication process, this results in the formation of not only calcium carbonate but also other calcium contacting minerals. However, despite the effect of the presence of the chitosan being evident in terms of the morphology of the resultant crystallites within the final composites in comparison to those obtained in the absence of the same, the mineral component was predominantly calcite.

The possibility of modifying the morphology of the resultant crystallites to form the desired pancake-like structure by addition of PAA was investigated, based on its successful application in the two-step fabrication process. The printing ink, in this case, was a blend of PAA, chitosan and $\mathrm{CaCO}_{3}$. The morphology of the resultant crystallites in the final 3D printed $\mathrm{Ch}-\mathrm{CaCO}_{3}$-PAA composite was modified to aggregates of $\mathrm{CaCO}_{3}$ needles with preferential outward growth, a structure different from that of the desired structure. Also, compared to chitosan hydrogel-based scaffolds mineralized via the McGrath method (in the presence of PAA), the addition of PAA did not improve the interaction (electrostatic/noncovalent interaction) between the polymer and the crystallites. This is to say that unlike the case of the two-step process, the SEM images (Figure 4.6) of $\mathrm{Ch}-\mathrm{CaCO}_{3}-\mathrm{PAA}$ the composites did not show the polymer network entangling with the crystallites at a nano or microscale. These composites lacked the strong polymer- $\mathrm{CaCO}_{3}$ interactions observed in the case of crystallites formed when using the two-step fabrication process. Thus, it is concluded that the single-step fabrication process offers limited control over the morphology of the resultant crystallites in the composite and the nature of their interaction with the organic component.

Another drawback of the protocol used in the single-step fabrication process is the leaching of inorganic content from the printed structure during the lamination process (addition of ethanolic $\mathrm{NaOH}$ ). Higher leaching was observed when using blended inks with high calcium carbonate loading (30-50\% at constant $\mathrm{pH})$. This is attributed to the variation in the dissolution-precipitation kinetics of calcium carbonate in the polymer matrix under a given $\mathrm{pH}$ condition. When the added calcium carbonate exceeds its solubility limit under a given $\mathrm{pH}$ condition the excess reprecipitates in the form of nano or micron-sized crystallites that 
are weakly bound to the organic matrix. Such weak interactions lead to leaching out of these crystallites during the lamination process when interacting with a base.

Furthermore, when using the single-step fabrication process, the variation in the dissolutionreprecipitation kinetics occurring as a function of the $\mathrm{pH}$ and the concentration of the initially added $\mathrm{CaCO}_{3}$ to the hydrogel during different steps of the fabrication process also affects the thermal decomposition behaviour of the final composite. The fabrication process is found to increase the thermal stability (in terms of the temperature at which the $2^{\text {nd }}$ degradation occurs) of the final composites generated irrespective of the initial concentration of calcium carbonate loading in the blended ink, compared to that of the corresponding blended inks. However, continued addition of $\mathrm{CaCO}_{3}$ in the blended ink did not change the thermal decomposition behaviour of the composites except by increasing the final percentage residual volume. Similar to the case of the composites developed via the two-step method discussed in Chapter 3, the residual volume is however not a measure of the actual mineral content of the final composite. Here, the variations in the dissolutionreprecipitation kinetics of calcium carbonate within the polymer matrix result in some chitosan being occluded with the $\mathrm{CaCO}_{3}$ crystallites and remain undecomposed, thus increasing the final residual volume after complete thermal decomposition.

TGA data for composites fabricated via the single-step method indicate that the actual mineral content (i.e., the residual volume) after thermal decomposition varies depending on the degree of leaching observed during the lamination process; and the plausible increase in the final residual volume due to undecomposed chitosan in the final composite. When the mineral loading in the blended ink is low such as $10 \%, 20 \%$ or $40 \% \mathrm{CaCO}_{3}$ loading (at respective $\mathrm{pH}$ conditions), the observed leaching is lower and thus the increase in final residual volume compared with that expected may be attributed to the presence of undecomposed organic matter along with the mineral content. When using blended inks with $30 \%$ or $50 \% \mathrm{CaCO}_{3}$ loading, high leaching occurs during the lamination process which dominates over the possible increase in the residual volume due to undecomposed occluded chitosan. Thus, it may be concluded that when the single-step fabrication method is used, in order to obtain composites with optimal mineral content and thermal stability, a balance between the concentration of calcium carbonate preloaded in the printing ink and the $\mathrm{pH}$ of the system has to be maintained such that the loss of minerals during the lamination process may be minimized. 
Hence, although the single-step fabrication process is less laborious and time-consuming, considering all of the above limitations, it is concluded that chitosan-calcium carbonate composites fabricated via the two-step process involving the 3D printing of chitosan hydrogel-based scaffolds using pure chitosan hydrogels as the printing ink and then mineralizing these via the McGrath method (in the presence of PAA), is a better approach towards developing chitosan- $\mathrm{CaCO}_{3}$ composites with tuneable properties. Thus, only this fabrication technique is used to develop composites for all further experiments. 


\subsection{Bibliography}

1. Huang, Z.-H., Y.-S. Dong, C.-L. Chu, and P.-H. Lin, Electrochemistry assisted reacting deposition of hydroxyapatite in porous chitosan scaffolds. Materials Letters, 2008. 62(19): p. 3376-3378.

2. Zhang, L.-J., X.-S. Feng, H.-G. Liu, D.-J. Qian, L. Zhang, X.-L. Yu, and F.-Z. Cui, Hydroxyapatite/collagen composite materials formation in simulated body fluid environment. Materials Letters, 2004. 58(5): p. 719-722.

3. Thein-Han, W. and R. Misra, Biomimetic chitosan-nanohydroxyapatite composite scaffolds for bone tissue engineering. Acta Biomaterialia, 2009. 5(4): p. 1182-1197.

4. Zhang, Y. and M. Zhang, Cell growth and function on calcium phosphate reinforced chitosan scaffolds. Journal of Materials Science: Materials in Medicine, 2004. 15(3): p. 255-260.

5. Serra, T., J.A. Planell, and M. Navarro, High-resolution PLA-based composite scaffolds via 3-D printing technology. Acta Biomaterialia, 2013. 9(3): p. 5521-5530.

6. Li, B., Q. Hu, X. Qian, Z. Fang, and J. Shen, Bioabsorbable chitosan/hydroxyapatite composite rod prepared by in situ precipitation for internal fixation of bone fracture. Acta Polymerica Sinica, 2002(6): p. 828-833.

7. Venkatesan, J. and S.-K. Kim, Chitosan composites for bone tissue engineering - an overview. Marine Drugs, 2010. 8(8): p. 2252-2266.

8. $\mathrm{Hu}, \mathrm{Q}$, B. Li, M. Wang, and J. Shen, Preparation and characterization of biodegradable chitosan/hydroxyapatite nanocomposite rods via in situ hybridization: a potential material as internal fixation of bone fracture. Biomaterials, 2004. 25(5): p. 779-785.

9. Cho, J., M. Joshi, and C. Sun, Effect of inclusion size on mechanical properties of polymeric composites with micro and nano particles. Composites Science and Technology, 2006. 66(13): p. 1941-1952.

10. Chen, B. and K. Sun, Poly (E-caprolactone)/hydroxyapatite composites: effects of particle size, molecular weight distribution and irradiation on interfacial interaction and properties. Polymer Testing, 2005. 24(1): p. 64-70.

11. Roeder, R.K., M.M. Sproul, and C.H. Turner, Hydroxyapatite whiskers provide improved mechanical properties in reinforced polymer composites. Journal of Biomedical Materials Research Part A, 2003. 67(3): p. 801-812. 
12. Allo, B.A., D.O. Costa, S.J. Dixon, K. Mequanint, and A.S. Rizkalla, Bioactive and biodegradable nanocomposites and hybrid biomaterials for bone regeneration. Journal of Functional Biomaterials, 2012. 3(2): p. 432-463.

13. Lee, K.-H. and S.-H. Rhee, The mechanical properties and bioactivity of poly (methyl methacrylate)/SiO $2-\mathrm{CaO}$ nanocomposite. Biomaterials, 2009. 30(20): p. 3444-3449.

14. Karimi, A.R. and A. Khodadadi, Mechanically Robust 3D Nanostructure ChitosanBased Hydrogels with Autonomic Self-Healing Properties. ACS Applied Materials \& Interfaces, 2016. 8(40): p. 27254-27263.

15. Declet, A., E. Reyes, and O. Suárez, calcium carbonate precipitation: a review of the carbonate crystallization process and applications in bioinspired composites. Reviews on Advanced Materials Science, 2016. 44(1).

16. Musumeci, A.W., R.L. Frost, and E.R. Waclawik, A spectroscopic study of the mineral paceite (calcium acetate). Spectrochimica Acta Part A: Molecular and Biomolecular Spectroscopy, 2007. 67(3-4): p. 649-661.

17. Lazzeri, A., S.M. Zebarjad, M. Pracella, K. Cavalier, and R. Rosa, Filler toughening of plastics. Part 1-the effect of surface interactions on physico-mechanical properties and rheological behaviour of ultrafine $\mathrm{CaCO}_{3} / \mathrm{HDPE}$ nanocomposites. Polymer, 2005. 46(3): p. 827-844.

18. Zhang, S. and K. Gonsalves, Synthesis of calcium carbonate-chitosan composites via biomimetic processing. Journal of Applied Polymer Science, 1995. 56(6): p. 687-695.

19. Reis, J.M.L.d., Effect of textile waste on the mechanical properties of polymer concrete. Materials Research, 2009. 12(1): p. 63-67.

20. Lapcik, L., P. Jindrova, B. Lapcikova, R. Tamblyn, R. Greenwood, and N. Rowson, Effect of the talc filler content on the mechanical properties of polypropylene composites. Journal of Applied Polymer Science, 2008. 110(5): p. 2742-2747.

21. Abert, J., A. Amella, S. Weigelt, and H. Fischer, Degradation and swelling issues of poly-(d, l-lactide)/ $\beta$-tricalcium phosphate/calcium carbonate composites for bone replacement. Journal of The Mechanical Behavior of Biomedical Materials, 2016. 54: p. 82-92.

22. Chevigny, C., N. Jouault, F. Dalmas, F. Boué, and J. Jestin, Tuning the mechanical properties in model nanocomposites: Influence of the polymer-filler interfacial interactions. Journal of Polymer Science Part B: Polymer Physics, 2011. 49(11): p. 781791. 
23. Swain, S.K., S. Dash, S.K. Kisku, and R.K. Singh, Thermal and oxygen barrier properties of chitosan bionanocomposites by reinforcement of calcium carbonate nanopowder. Journal of Materials Science \& Technology, 2014. 30(8): p. 791-795. 


\section{- Mechanical Properties of Chitosan Hydrogel-based Scaffolds}

\subsection{Introduction}

3D chitosan-calcium carbonate (chitosan- $\mathrm{CaCO}_{3}$ ) composites developed in this research are envisioned as future bone graft materials, hence it is necessary to investigate the mechanical performance of these materials within a physiologically relevant mechanical regime (stress/strain and loading rate). It is important to understand the significance of the structure (architecture and porosity), as well as the contribution of the composition of the composite (inorganic and organic components) to the final mechanical properties of the material. In this Chapter, the behaviour of the $3 \mathrm{D}$ chitosan- $\mathrm{CaCO}_{3}$ composite materials under static and dynamic compressive stress loading conditions, while maintaining the structural parameters of the original chitosan scaffold (porosity and architecture) constant is described. This enables the contribution of the inorganic/organic phases of the composite to the overall mechanical property material to be ascertained.

It is well-established that the mechanical strength of biopolymer-based tissue engineering materials such as chitosan, collagen or gelatin is inherently very low [1-3]. It is for this reason that many of these naturally-derived hydrogel biomaterials are not considered suitable for bone tissue engineering applications. Methods adopted to increase the strength of such polymer-based systems include increasing the concentration of the biopolymer or introducing various modifications such as increasing the type and density of crosslinks in the polymer chains. Such enhancements, however, affect the biological performance of the resultant materials in terms of the possibility of increased toxicity, limited diffusibility and hindrance to cell migration in vivo [4-6]. Addition of mineral particles to the biopolymers as fillers has been identified as an alternative method to circumvent these disadvantages while improving the mechanical strength of biopolymers via enhanced interactions and better stress distribution between the fillers and the organic component [4].

Chitin/chitosan hydrogel-based composites incorporating a wide range of filler materials such as calcium phosphates (especially hydroxyapatite), bioglass, graphene, carbon nanotubes, and silica/zirconia have been studied for bone tissue engineering applications [7- 
12]. Reports suggest that these composites show excellent biocompatibility and osteoconductivity and enhanced mechanical strength in comparison to pure chitosan. Most of these composites are developed by dispersing (mechanical mixing) the filler particles directly into the hydrogel (as discussed in Chapter 4) and then freeze-drying the resultant composite to form 3D structures. For example, Chang et al. reported on nano-sized hydroxyapatite-chitin hydrogel-based composites with a porous structure that exhibited a compressive strength of $274 \mathrm{kPa}$, almost 10 fold higher than that of pure chitin-based materials [13].

Chesnutt et al. reported a compressive strength of $117.6 \pm 52.8 \mathrm{MPa}$ for chitosan hydrogelnanohydroxyapatite-based composites that were developed via co-precipitation of hydroxyapatite crystallites and chitosan hydrogel in the form of solid beads, an order of magnitude higher than pure chitosan hydrogel-based structures and almost three orders of magnitude higher than those produced via blending of fillers and polymers mechanically as in the previous example [14].

The McGrath mineralization method, like the above-described method, is a non-traditional method developed in order to fabricate inorganic-organic composites based on chitosan and calcium carbonate. It is therefore anticipated that 3D printed composites developed via the McGrath method will also result in enhanced mechanical properties compared with the nonmineralized scaffolds. Hence, it is important to characterize the mechanical behaviour of these composites and identify the factors that aid in controlling their mechanical strength.

Studies have shown that the mechanical properties of bone vary with the anatomical location, the degree of mineralization, porosity, apparent density, moisture content and age of the specimen [15]. The exceptional mechanical strength of bone (refer to Chapter 1, Table 1.1), despite the high mineral content and porosity, is attributed to the hierarchal organization and the resulting enhanced interactions between the mineral component and the organic matrix $[16,17]$. The enhanced interaction is also facilitated by the fact that the minerals (hydroxyapatite) are initially formed within the collagen matrix (refer to Chapter 1, Section 1.2.2 (a, b)) [18]. Similarly, the high mechanical strength of nacre, which is comparable to that of bone, despite a much higher mineral content ( $95 \%)$, is also attributed to, an albeit simpler, hierarchal organization of the organic and the inorganic components in the so-called brick-mortar architecture of aragonitic platelets within a chitin-based organic matrix (refer to Chapter 1, Section 1.2.3 (a, b)) [19, 20]. These examples from nature direct more investigations to ascertain whether the mechanical strength of 
biopolymer matrices can be enhanced by incorporating filler particles that are hierarchically organized along with the organic matrix, and show enhanced interaction with the matrix, thus replicating such extraordinary properties seen in the native tissue in laboratory conditions.

Fabrication of 3D composites via 3D printing using chitosan hydrogel as the printing ink followed by mineralization using the McGrath method in the presence of polyacrylic acid (PAA) as the crystal growth modifier was described in Chapter 3. Hierarchically structured 3D composites replicating nacre on a macroscale were developed using this mineralization method wherein numerous nanosized $\mathrm{CaCO}_{3}$ crystallites were observed to aggregate in association with the chitosan hydrogel matrix to form micron-sized calcium carbonate crystallites. In some cases, the crystallites were found to show oriented aggregation similar to the hydroxyapatite platelets orienting within collagen fibrils during initial stages of bone formation [21]. This is to say, that materials portraying several of the structural features similar to that of bone and nacre, which have been shown to be critical in defining their (bone and nacre) mechanical behaviour, have been fabricated in this research. In this Chapter, the enhancements and or changes in the mechanical properties of the chitosan hydrogel-based 3D composite structures as a result of this hierarchal growth are investigated.

The mechanical behaviour of bone is well characterized under both static as well as dynamic stress-loading conditions $[16,22]$. The dynamic properties are more relevant in terms of the comparison to the in vivo stress loading that the skeletal system is subjected to on a daily basis during real life activities such as during walking, running, exercising and under extreme impact conditions that could potentially result in fracture of the bone [23].

The mechanical properties of polymers and almost all biological tissues are dependent on their viscoelastic tendencies. These properties are advantageous in regions subjected to constant physical stress such as the skeletal system. Developing an understanding of the nature of energy absorption and dissipation during normal, as well as high impact stress is beneficial in identifying the mechanism of failure propagation and hence aiding in developing appropriate graft materials. For example, the viscoelastic properties of trabecular bone, with a modulus of elasticity of $380 \pm 224 \mathrm{MPa}$ (knee bone samples compressed to $0.6 \%$ strain in compression), has an effective energy absorptive property within its elastic range of deformation, which may be helpful in protecting bone and articular cartilage during normal stress loading conditions [24]. 
Viscoelasticity is a combination of viscosity, the tendency to flow and elasticity, the tendency to revert to the material's original state. Unlike metals which have an instantaneous elastic response to stress loading and complete recovery upon unloading, composites based on organic polymers tend to exhibit a delayed/gradual deformation and recovery pattern. The response is time-dependent and is also affected by the rate of stress or strain loading [25]. The relation between stress and strain for elastic (Eq 3.1) and viscoelastic materials shows that the fluid-like tendency of the latter introduces a timedepended strain variable (Eq 3.2).

$$
\begin{gathered}
\sigma=E \varepsilon \\
\sigma=\sigma\left(\varepsilon, \varepsilon^{*}\right)
\end{gathered}
$$

According to Equation 3.1 for an elastic material, the applied stress $(\sigma)$ is directly proportional to the applied strain $(\varepsilon)$ within the elastic limit of the material and the proportionality constant $E$ is a measure of its elastic modulus. However, in the case of a viscoelastic material the stress is dependent not only on the strain but also on the time dependent strain function $\varepsilon^{*}=d \varepsilon / d t$. Hence, in the case of polymer-based composites, the response to an applied stress will depend on the rate of the applied stress (how slow or fast). Therefore, the stress-strain relationship will not be unique, and it will vary as a function of the time or the rate of applied stress and the corresponding strains. When a viscoelastic material is strained, part of the strain energy (energy supplied during deformation) is stored as potential energy which is used for recovery and the remainder is dissipated as heat.

The viscoelastic properties of a material can be studied by applying an oscillatory force to the material and measuring the resulting oscillatory deformation [26]. In the case of viscoelastic materials, the applied stress and the resultant strain will be out of phase. The modulus is then expressed as a complex modulus $\left(E^{*}\right)$ of the storage $\left(E^{\prime}\right)$ and loss $\left(E^{\prime \prime}\right)$ moduli. The storage modulus is a measure of the energy stored in the material while stressed within the elastic range and the loss modulus is a measure of the energy dissipated when stressed beyond the yield point or the elastic limit. The relationships are expressed as:

$$
\begin{aligned}
& E^{*}=E^{\prime}+i E^{\prime \prime} \\
& E^{\prime}=\frac{\sigma_{0}}{\varepsilon_{0}} \cos \delta \\
& E^{\prime \prime}=\frac{\sigma_{0}}{\varepsilon_{0}} \sin \delta
\end{aligned}
$$




$$
\tan \delta=\frac{E^{\prime \prime}}{E^{\prime}}
$$

where $\sigma_{0}$ and $\varepsilon_{0}$ are the maximum stress and strain and $\delta$ is the phase angle difference between the applied stress and the resultant strain. $\operatorname{Tan} \delta(\mathrm{Eq} \mathrm{3.6)}$ is a measure of how efficiently the material loses energy due to molecular arrangements and internal friction when under stress. Any of these three parameters can be used to study the viscoelastic behaviour of a material.

For ideal elastic materials $\tan \delta=0$, whereas viscous materials exhibit high values of $\tan \delta$. Tan $\delta$ for cortical bone is reported to be in the range 0.01-0.04 depending upon the testing mode ( 3 point bending, torsional or shear), moisture content, the origin of the specimen, orientation and the strain rate [27]. Studies suggest that the dynamic properties of bone result from a combined interplay of the organic components, minerals and the water content; although the major contribution to the viscoelastic tendencies is attributed to the organic matrix [28].

This Chapter investigates various factors affecting the mechanical properties of chitosan$\mathrm{CaCO}_{3}$ composite materials fabricated by mineralization of $3 \mathrm{D}$ printed scaffolds via the McGrath method, in their dried state. Herein,

(a) mechanical properties such as compressive modulus, ultimate strength and the yield strength are calculated for pure chitosan hydrogel-based 3D scaffolds under uniaxial compression in static mode. The effect of varying the total scaffold porosity on these properties is also analysed;

(b) the effect of increasing $\mathrm{CaCO}_{3}$ content and varying the morphology of the $\mathrm{CaCO}_{3}$ crystallites within the final chitosan- $\mathrm{CaCO}_{3}$ composite, on their mechanical properties is analysed via uniaxial compressive loading in static mode and using a dynamic mechanical analyser in dynamic stress loading mode under increasing loading frequencies; and

(c) the nanoscale hardness at different regions within the 3D printed chitosan scaffolds and the composites is identified and compared to natural bone.

The observations from the structural and compositional analysis of chitosan- $\mathrm{CaCO}_{3}$ scaffolds described in Chapter 3 are used here to discuss the mechanical properties of the respective materials. The percentage $\mathrm{CaCO}_{3}$ content of the composites that were tested for mechanical properties was quantified via thermogravimetric analysis (TGA). As described in Chapter 3 the value thus obtained is an approximation of the total mineral content within 
the composite scaffold and is used merely as an upper limit of the same. The mechanical properties under compression were studied using two different instruments: uniaxial unconfined compression testing and a dynamic mechanical analyser (DMA). 


\subsection{Materials and methods}

3D chitosan hydrogel-based scaffolds were fabricated using the same materials and methods as described in Chapter 2. The 3D printed scaffolds were then mineralized via the McGrath method (in the presence of $2.5 \%$ PAA w.r.t dry weight of chitosan used) as explained in Chapter 3. All composites and chitosan hydrogel-based scaffolds (non-mineralized) were air-dried prior to mechanical testing and all experiments were performed in an air atmosphere at room temperature and humidity conditions unless otherwise mentioned.

The as fabricated 3D chitosan hydrogel-based scaffolds were cylindrical structures $15 \mathrm{~mm}$ in diameter $\times 16 \mathrm{~mm}$ in height in their wet state, however, mineralization and dimensional shrinkage upon air-drying, meant that the final dimensions of the dried samples varied from sample to sample. Hence, all the dried samples were hand-filed in an effort to maintain comparability in the dimensions across samples and also to ensure that the area in contact with the compression plates during testing was as parallel to the platen surface as practically possible. After air-drying and filing the dimensions of the samples varied within the range of $6.5 \pm 0.3 \mathrm{~mm}$ in diameter $\times 2.7 \pm 0.3 \mathrm{~mm}$ in height. The dimensions (diameter and height) of the tested samples were measured by Vernier callipers (four measurements are recoded and averaged) and the area of contact with the load cell for each sample tested under compression was calculated by analysing the optical images of samples taken before testing using Image J. Using the software, the surface area of porous samples (that is in contact with the load cell) was calculated by subtracting the total surface area of pores from the total surface area of the of the scaffolds ( $\pi r^{2}$ where $r$ is the radius of the scaffold).

It should be noted the dimensions of the samples used for mechanical testing are not in accordance with ASTM standards for biomedical scaffolds. This is due to the limitations of the fabrication process (semi-automated 3D printer) which would require significant time investment in order to 3D print chitosan hydrogel-based scaffolds of larger dimensions such that the final air-dried scaffolds (after shrinkage) would be (a) large enough to be handled during hand-filing and (b) that the final filed structure complies with the standard testing protocol. However, since the sample dimensions $(6.5 \pm 0.3 \mathrm{~mm}$ in diameter $\times 2.7 \pm 0.3 \mathrm{~mm}$ in height) are maintained to be as constant as possible across all samples studied, it is assumed that, any variations in the mechanical properties introduced as a result of this deviation from the standard protocol, would be the same across all samples tested under comparable testing parameters and instruments used and thus should be minimised within the limitations of the sample preparation. 


\subsubsection{Uniaxial unconfined compression testing}

Static loading: Tests were performed using an MTS Criterion ${ }^{\circledR}$ model 42 benchtop instrument. Initially, two different load cells were used to analyse the suitability of individual load cells to study the properties of the test samples under consideration. A set of five different chitosan hydrogel-based scaffolds were tested using both load cells. Scaffolds were compressed between 0-4 N (corresponding to 20\% strain) when using a 5 $\mathrm{N}$ load cell and to $75 \%$ strain when using a $5 \mathrm{kN}$ load cell, at a crosshead speed of $0.1 \mathrm{~mm} / \mathrm{s}$. A load limit of $4.9 \mathrm{~N}$ and $490 \mathrm{~N}$ respectively was introduced into the test protocol when using the respective load cells as safety measures to ensure that the load cell is not damaged. A preload force of $0.01 \mathrm{~N}$ was applied on the samples when using the $5 \mathrm{~N}$ load cell and $2 \mathrm{~N}$ was applied when using the $5 \mathrm{kN}$ load cell at a cross head speed of $0.1 \mathrm{~mm} / \mathrm{s}$ depending on the lower limit of sensitivity of individual load cells as prescribed by the manufacturer. Preloading is a process whereby the test sample is compressed to a known limit before beginning the actual compression testing. Here, this process was applied to ensure that a proper contact is established between the sample surface and the compression plates as the samples were hand-filed and may have high points on the surface that can cause irregularities in the data collected. Based on the stress-strain data obtained, the load cell that enabled determination of both elastic and plastic regions within the stress-strain plot of all materials tested, thus allowing for the full mechanical characterization the test samples to be quantified in terms of determining the compressive modulus, yield strength and compressive strength, was selected for use in all further testing.

As per the instrument's software design, during data collection, the software assigns a value equal to zero to the extension recorded by the load cell once the set preload force is attained. However, since an initially applied preload will cause some strain in the scaffolds, in order to also consider this strain in the calculations, a preload correction was performed such that the engineering strain calculated is a non-zero value at the beginning of data collection after the preload is reached. The preload correction was performed as per the video tutorial in reference [29].

Based on the results (discussed further in Section 5.3.1.1) a $5 \mathrm{kN}$ load cell was chosen as the appropriate load cell to study the mechanical properties under compression for chitosan hydrogel-based scaffolds and the corresponding composites. Five replicates of each type of sample were tested according to the test parameters described earlier for the $5 \mathrm{kN}$ load cell. 


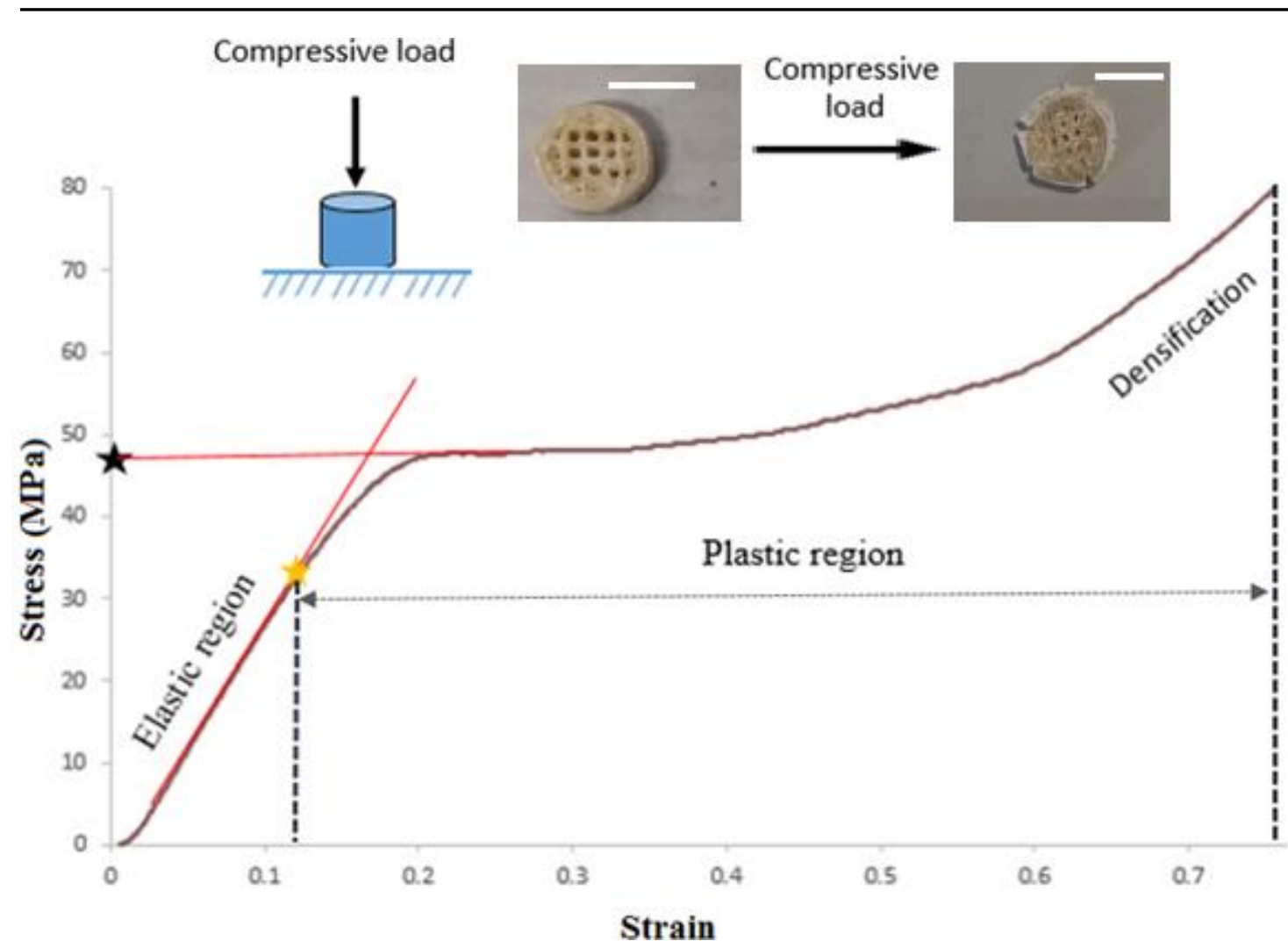

Figure 5.1: A representative stress-strain plot for chitosan-CaCO $\mathrm{C}_{3}$ composites under static compressive loading using the $5 \mathrm{kN}$ load cell after applying the preload correction in order to consider the strain induced due to the applied $2 \mathrm{~N}$ preload. The yellow icon represents the yield strength and the black icon shows the ultimate strength calculated based on the stress-strain plot. The initial part of the plot until the yield point is the elastic region and the region in between the dashed line shows the plastic region. Also shown are optical images of a composite sample before and after compression to $75 \%$ strain as a representative example of the crushing phenomenon. Scale bar is $0.5 \mathrm{~cm}$.

The stress-strain plot for every structure studied in this research showed three distinct regions as depicted in the representative stress-strain plot shown in Figure 5.1, similar to those reported for synthetic biopolymer-based composites and bone under compression [22, $30,31]$. In the case of the materials investigated here, in the first region, the elastic region, the applied stress is proportional to the resultant strain and the elastic modulus can be calculated from the slope. The point of deviation from proportionality corresponds to the yield point (yellow icon in Figure 5.1) and the yield strength (stress) is estimated from this value. During compression testing, due to filling of fractures as the stress loading progresses beyond the elastic limit of the structures, it becomes difficult to pinpoint a value of stress at which complete fracture occurs; usually depicted by a sudden drop in the load (as would be observed in tensile testing or in compression testing of brittle materials). Thus, the 
compressive strength of the samples could not be accurately calculated. Hence, the yield strength was calculated as the stress at which a sample deviates from its linear behaviour (the Hookean/ elastic region) and begin to yield. This was estimated by line fitting to the initial linear region of individual stress-strain plots of each sample (represented by the fitting line shown in the elastic region of the stress-strain plot in Figure 5.1), the point at which the plot deviates from this linear fit is denoted as the yield point, the stress corresponding to this point is the yield strength. Unlike, the standard procedure used in classical mechanics, where the yield stress is calculated by fitting a line to the linear elastic region at a fixed offset strain of $1 \%$ or $5 \%$, the data reported here are not based on a fixed offset strain. This is because the elastic range varied from sample to sample and in some cases, the point of intersection between the fitting line at $1 \%$ or $5 \%$ offset strain and the stress-strain curve lay in the plastic region.

Beyond the yield point, a second linear region is defined that has a reduced slope compared to the first region. This region depicts the initiation of plastic deformation of the sample being compressed and also indicates the initial collapse of the scaffold under the applied stress. This region is characterized by large changes in the resultant strain with small increments in the applied load. Further compression causes densification of the scaffold by fracture filling, this region shows a further increase in the slope of the stress-strain curve, with the sample being compressed to a flat disk (Figure 5.1). Other than these observed regions and parameters, literature also cites yet another parameter called the ultimate strength that can be identified from the stress stain plot as the highest stress that can be sustained by a sample after yielding but before crushing and densification (black icon in Figure 5.1). Hence, for comparison with other materials, the ultimate strength has been calculated for all mechanically tested samples.

\subsubsection{Dynamic mechanical analysis}

The viscoelastic properties of the composites were analysed using a dynamic mechanical analyser (DMA) (simultaneous differential thermal analysis (SDTA) $861^{\mathrm{e}}$, Mettler Toledo, $\mathrm{GmbH}$, Switzerland) operated by STA-R ${ }^{\mathrm{e}}$ version 9.10 software in compression mode. To analyse the effect of increasing loading frequency on the viscoelastic behaviour of our materials, compressions were performed under a dynamic stress applied at varying loading frequency of $1-15 \mathrm{~Hz}$ with a $0.5 \mathrm{~Hz}$ increment in a $37^{\circ} \mathrm{C}$ air atmosphere. The maximum loading force was set to $12 \mathrm{~N}$ and the displacement amplitude to $5 \mu \mathrm{m}$ (assuming that the strain thus induced by the $40 \mathrm{~N}$ load cell within the DMA machine falls within the elastic 
limit of the tested samples, based on the stress-strain response obtained from the uniaxial compression tests performed using a $5 \mathrm{kN}$ load cell, the dimensions and porosity of the samples used in both tests are the same). Five replicates of each type of sample were tested.

\subsection{3: Hardness testing: nanoindentation}

Nanoindentation is a relatively simple non-destructive technique that has recently gained popularity as an effective hardness testing technique for composites in preference to microhardness testing. In particular, it is used to determine mechanical properties such as elastic modulus, yield strength approximation, and hardness over very small length scales $[32,33]$. In the case of chitosan hydrogel-based samples, nanoindentation is used to determine the hardness at the nanoscale at different regions within the $3 \mathrm{D}$ structure such as its struts and nodes (where the struts overlap) and is compared to that of natural bone.

Hardness testing typically involves driving an indenter tip of known area through a test sample and calculating the hardness by dividing the maximum load applied by the size of the residual plastic impression left by the indenter on the sample, measured using optical image analysis. In the case of nanoindentation, the residual area is often in the range of microns and is difficult to measure via traditional methods. Hence, in this technique the depth of penetration of the indenter beyond the sample surface is measured simultaneously as the load is applied and using an indenter of known area, the size of the residual impression is calculated based on the known indentation depth.

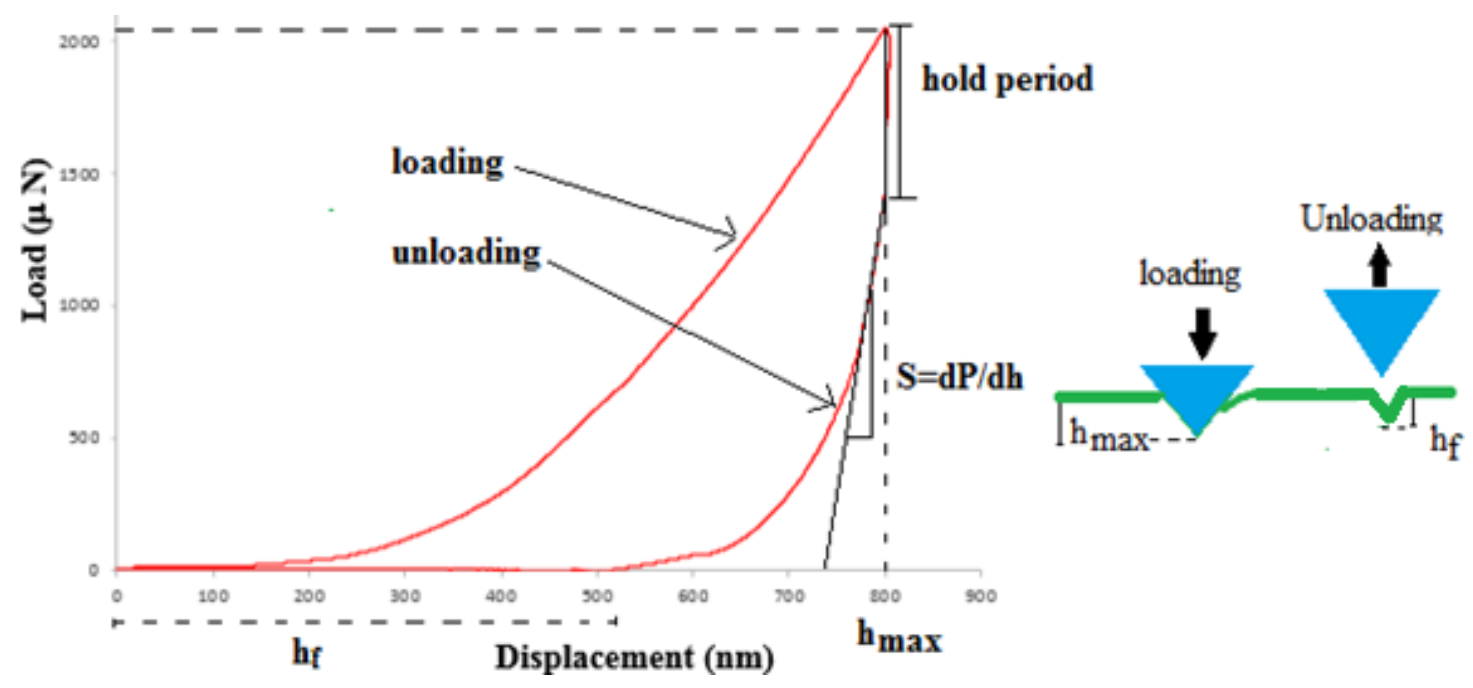

Figure 5.2: A typical load-displacement curve for a chitosan-CaCO 3 composite scaffold for indentations performed in constant displacement mode. $P_{\text {max }}$ is the maximum load reached at $h_{\text {max }}$ the maximum indentation depth, $S$ is the contact stiffness and $h_{f}$ is the final/residual depth after unloading. 
Nanoindentation is the only test where information can be obtained about both the plastic as well as the elastic response of a material at the nanoscale. Figure 5.2 shows a typical load-displacement curve for a representative chitosan hydrogel-based composites when indentation is performed in displacement control mode (i.e. the max indentation depth is set to a value and the applied load is varied). The elastic modulus is usually calculated based on the slope of the unloading curve, which is representative of the elastic response of the material, given by $E_{r}$ the residual modulus at the indentation contact as given below

$$
E_{r}=\frac{\sqrt{\pi}}{2} \frac{d p}{d h} \frac{1}{\sqrt{A}}
$$

where $A$ is the projected contact area at maximum load, and $\frac{d p}{d h}$ represents the contact stiffness. This can be related to the test sample's elastic modulus (E) using the Oliver and Pharr relation

$$
\frac{1}{E_{r}}=\frac{1-v^{2}}{E}+\frac{1-v_{i}^{2}}{E_{i}}
$$

where $v_{i}$ and $E_{i}$ are Poisson's ratio and the elastic modulus of the indenter tip respectively and $v$ is Poisson's ratio of the test sample. When the indenter stiffness is much higher than that of the test sample, the hardness $H$ can be calculated as

$$
H=\frac{P_{\max }}{A}
$$

where $P_{\max }$ is the maximum load reached at the maximum indentation depth and $A$ is the projected contact area at that depth.

However, it must be noted that this modulus may not be comparable to the elastic modulus calculated from other testing methods as the nanoindentation load-displacement curve does not account for pileup. The indentation elastic modulus may be affected greatly by material behaviour (e.g. piling-up) that is not accounted for in the analysis of load-displacement data $[32,34]$. The data obtained are thus only comparable to nanoindentation data obtained under similar experimental conditions and using the same instrument.

To measure the hardness of chitosan hydrogel-based samples (non-mineralized and the composite variants), nanoindentation tests were performed using a Hysitron TI-950 TriboIndenter (Hysitron Inc) fitted with a Berkovich diamond tip (50-100 nm). All samples were filed using sandpapers of different grit sizes and finally polished using a 4000-grit 
silicon carbide polishing paper. Prior to testing, the probe was calibrated using a standard fused quartz sample provided with the instrument by the manufacturer. The test samples were then mounted on the sample stage using superglue while ensuring that the top surface of the sample (where indentation occurs) does not interact with the glue and hence the properties (hardness, modulus) are not affected. Also, it is important to note that all indentations were performed in a direction perpendicular to the layer-laydown axis of the 3D scaffold.

Testing was performed in displacement control mode by maintaining the indentation depth at $800 \mathrm{~nm}$ (maximum load recorded was about $8 \mathrm{mN}$ ) for $5 \mathrm{~s}$ and then releasing. The reduced modulus $E_{r}$ and the hardness $H$ were calculated using the inbuilt software using the OliverPharr analytical method. The resultant properties are analysed based on an average of more than 15 indentations are performed at different regions of a single test sample. Some indentations resulted in hardness values approximately three times higher than the rest of the measurements obtained on that sample. It was concluded that these indentations must have occurred on a crystal on the polymer surface, hence resulting in a high harness value. Since these values were not representative of the actual polymer-mineral composite, these were not considered for calculating the mean hardness value for that respective sample.

\subsection{4: Statistical analysis}

Statistical analysis was performed using PRISM 6 graph pad software via one-way ANOVA variance testing to analyse the variation in the yield strength, ultimate strength and compression modulus among samples with varying mineral content. A Tukey post hoc analysis $(p<0.05)$ was performed to identify the point of variance. The unpaired t-test $(p<0.05)$ was used to identify the significance of the variation in the mechanical properties with the porosity as only two different porosities were considered. 


\subsection{Results and discussion}

The appropriate testing parameters to be used for the uniaxial compression testing of chitosan hydrogel-based scaffolds and the composite variants were identified first. The effect of increasing the total porosity on the mechanical properties of chitosan hydrogelbased scaffolds were then studied using 3D samples with two different macropore dimensions that are obtained when as-fabricated chitosan hydrogel-based scaffolds with 1.5 $\mathrm{mm} \times 1.5 \mathrm{~mm}$ or $2.0 \mathrm{~mm} \times 2.0 \mathrm{~mm}$ macropores (in wet condition) are subject to air-drying prior to uniaxial compression testing using the testing parameters identified. The actual dimensions and porosity of the resultant air-dried scaffolds were measured prior to testing.

The stress-strain response of air-dried chitosan- $\mathrm{CaCO}_{3}$ composites, developed by mineralization of as-fabricated chitosan hydrogel-based scaffold with $1.5 \mathrm{~mm} \times 1.5 \mathrm{~mm}$ macropores via the McGrath method, as a function of (1) increasing percentage $\mathrm{CaCO}_{3}$ content and (2) the morphology of the resultant calcium carbonate crystallites were studied. The porosity analysis discussed in Chapter 3 revealed that there is very little difference in the porosities of air-dried chitosan hydrogel-based scaffolds and composites developed via different mineralization methods. Thus, since these parameters are maintained constant within the limitations of the sample preparation for all samples (mineralized and nonmineralized), any variations observed in the data collected are attributed to the intrinsic properties of the composite such as the percentage $\mathrm{CaCO}_{3}$ content, the size and morphology of the crystallites, and the intrinsic properties of the organic matrix.

It must be noted that the stress-strain relationship described herein is based on the engineering stress and strain calculated from the force-displacement data collected.

\subsubsection{Static loading: identifying testing parameters}

In static loading, test samples are compressed at a constant crosshead speed corresponding to a displacement rate of $0.1 \mathrm{~mm} / \mathrm{s}$ and the load applied is measured using different load cells. The strain is calculated based on the displacement of the crosshead as the sample is being compressed. As the physical size of the samples that were used for this test was very small, attaching an extensometer to collect more precise displacement data was not possible. Thus, the material response was normalized against sample geometry in order to make a direct comparison. Therefore, the dimensions of each sample were measured using Vernier callipers prior to the mechanical testing. These dimensions were also ascertained by analysis of the optical images of the samples taken prior to testing using Image J. 
Factors affecting the elastic modulus, yield strength and the compressive strength of biocomposites like bone vary with structural properties such as pore architecture, pore distribution, cross-sectional area and shape of the tested samples; testing parameters such as direction of the applied load, mode of testing (compression, tension or shear), strain rate, and whether multidirectional or unidirectional stress is applied; and material properties, such as composition, mineral/filler content and the apparent density of the test specimen [35]. For example, the compressive strength of wet cortical bone is about $193 \mathrm{MPa}$ (along) and $133 \mathrm{MPa}$ (normal) along different loading axes and is mostly considered to be a transversely isotropic material [36]. In the case of trabecular bone, the elastic modulus reaches a maximum of $500 \mathrm{MPa}$ and the compressive strength is dependent on the apparent density of the specimen as it varies across different regions of the skeletal system [37].

The compressive strength and modulus of bone have also been shown to vary with the strain rate/crosshead speed revealing that cortical bone, which is almost non-porous, changes from ductile to brittle behaviour with increasing strain rate. This effect being prominent in defining the compressive strength as opposed to the modulus [35]. Uniaxial compression tests performed on chitosan hydrogel-based scaffolds and composites were undertaken until failure using a higher value of compressive crosshead speed: $0.1 \mathrm{~mm} / \mathrm{s}\left(0.05 \mathrm{~s}^{-1}\right.$ strain rate), compared to the usual $0.01 \mathrm{~mm} / \mathrm{s}$ as the former value is closer to those used to simulate trauma and impact failure in bone $\left(0.1 \mathrm{~s}^{-1}\right.$ strain rate), such that a higher limit to fracture resistance under compressive stress can be determined for chitosan hydrogel-based structures [38].

Accordingly, to obtain information about the most suitable load cell that can be used to study the mechanical properties of air-dried chitosan hydrogel-based scaffolds and the corresponding composite variants, air-dried chitosan hydrogel-based (3D printed with 1.5 $\mathrm{mm} \times 1.5 \mathrm{~mm}$ macropores in wet state) scaffolds were compressed uniaxially to $75 \%$ strain using two different load cells at a crosshead speed of $0.1 \mathrm{~mm} / \mathrm{s}$ and the mechanical properties of the scaffolds were analysed in each case.

When using a $5 \mathrm{~N}$ load cell, it was however found that within the load limit $(4.9 \mathrm{~N})$ the test sample could only be compressed to about $20 \%$ strain (Figure 5.3) and compressions beyond this would result in damaging the load cell. The resultant stress-stain response showed a linear curve that corresponds to the elastic deformations of the tested sample. Thus, allowing only the compressive modulus of chitosan hydrogel-based scaffolds to be calculated. This suggests that the stiffness of our samples is much higher than the prescribed stiffness limit 
for test samples when using a $5 \mathrm{~N}$ load cell. The average modulus as calculated from the slope of the linear region of the stress-strain plot is $0.6 \pm 0.1 \mathrm{MPa}$. Figure 5.3 shows representative load-displacement and stress-strain plots for chitosan hydrogel-based scaffolds compressed using a $5 \mathrm{kN}$ or a $5 \mathrm{~N}$ load cell.

When using a $5 \mathrm{kN}$ load cell, the stress-strain response of the scaffolds over a higher range of stress could be obtained. Accordingly, the samples were compressed until $75 \%$ strain (prior to attaining the load limit) and were found to undergo complete failure. The average compressive modulus determined from the slope of the linear elastic region is $232 \pm 45$ MPa. This is approximately 350 times higher than the modulus calculated in the case of chitosan hydrogel-based scaffolds when using a $5 \mathrm{~N}$ load cell. This value appeared more sensible compared to those obtained when using a $5 \mathrm{~N}$ load cell based on literature, which indicates that freeze-dried chitosan hydrogel-based scaffolds have a compressive moduli 0.6 $\pm 0.1 \mathrm{MPa}[39]$. It has already been shown in Chapter 2 that freeze-drying the samples results in spongy and low strength scaffolds. This suggests that when using a $5 \mathrm{~N}$ load cell there is limited scope for obtaining the complete elastic response of the chitosan hydrogel-based scaffolds under the testing conditions (crosshead speed) used. Hence, based on these results, all further compressive testing was performed using a $5 \mathrm{kN}$ load cell.

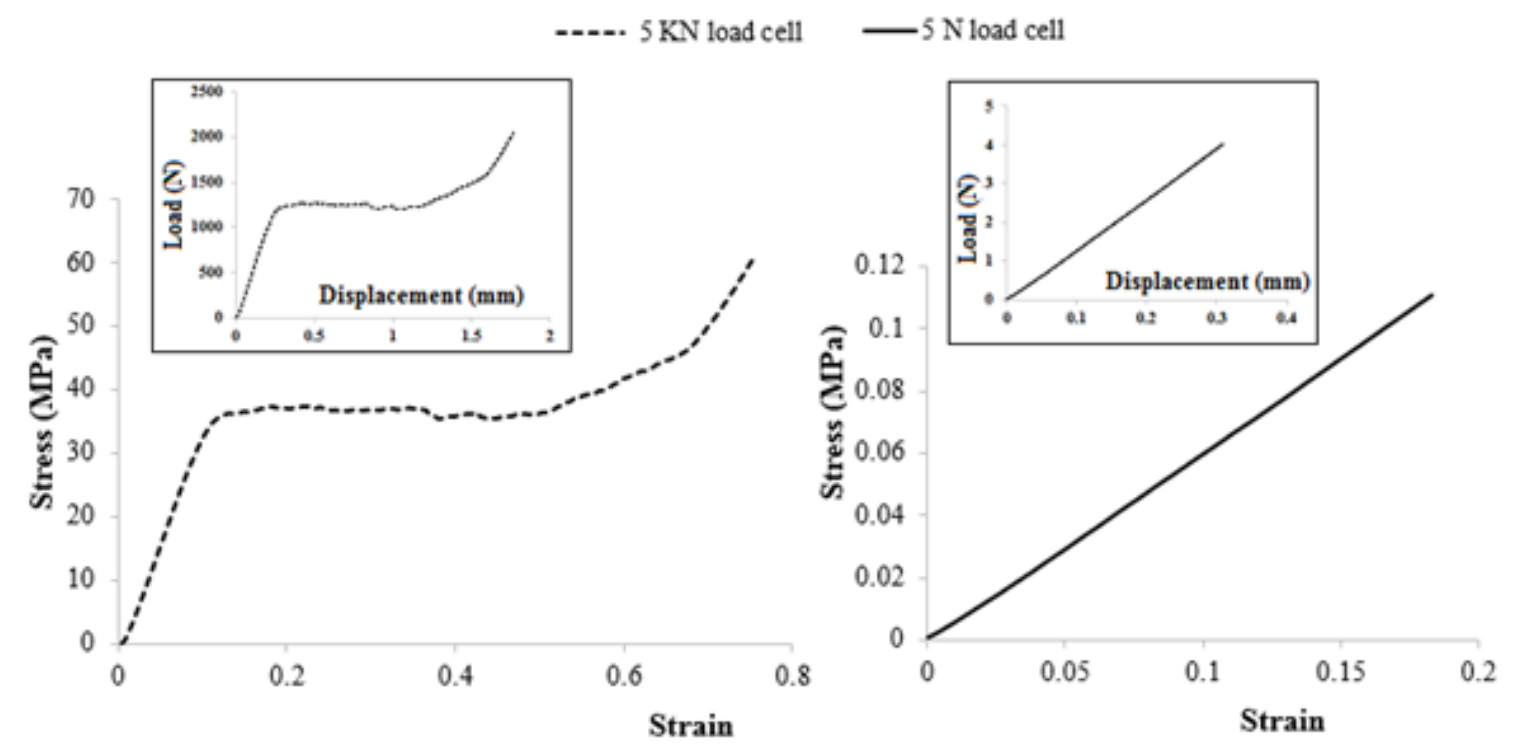

Figure 5.3: Representative stress-strain plots for chitosan hydrogel-based scaffolds under compression. Insets are the corresponding load displacement plots. Different scaffolds were used for the two tests. 
The variation in the mechanical properties under uniaxial compressive loading in static mode, with respect to:

(a) the porosity of the final air-dried scaffolds; and

(b) the presence of calcium carbonate crystallites within the hydrogel matrix introduced via the McGrath mineralization method

were further analysed.

\subsection{1a Effect of porosity}

In this experiment, chitosan hydrogel-based scaffolds with two different macropore porosities, obtained by air-drying as-fabricated chitosan hydrogel-based scaffolds with two different macropores dimensions namely $1.5 \mathrm{~mm} \times 1.5 \mathrm{~mm}$ or $2.0 \mathrm{~mm} \times 2.0 \mathrm{~mm}$, were fabricated. These were then hand-filed, and the mechanical properties were studied via uniaxial compression testing in static mode to $75 \%$ strain using a $5 \mathrm{kN}$ load cell at a crosshead speed of $0.1 \mathrm{~mm} / \mathrm{s}$. Five replicates of each type of sample were tested. In the case of chitosan hydrogel-based scaffolds with $1.5 \mathrm{~mm} \times 1.5 \mathrm{~mm}$ macropores (in its wet state), air-drying resulted in shrinkage of the macropores and the final pores size was $0.62 \pm 0.01$ $\mathrm{mm}$. Similarly, the final pore size after air-drying of scaffolds with $2.0 \mathrm{~mm} \times 2.0 \mathrm{~mm}$ macropores was $0.99 \pm 0.13 \mathrm{~mm}$.

In general, literature suggests that the mechanical strength of any structure decreases upon increasing porosity [40-42]. Although there is large variation in the pore dimensions of the two types of samples chosen were in the dried state), based on the porosity analysis discussed in Chapter 2, their estimated porosity did not vary largely; wherein, the porosity of chitosan hydrogel-based scaffolds with $0.62 \pm 0.01 \mathrm{~mm}$ macropores is $51.6 \pm 1.3 \%$ and that of the scaffolds with $0.99 \pm 0.13 \mathrm{~mm}$ macropores is $58.8 \pm 1.2 \%$. This is also reflected in the stress-strain data for the tested samples (in dried state) with the different two porosities (Figure 5.4 (Table 5.1 shows a representative set of data obtained for one sample of each type)) indicating that the variation in the average compressive moduli, yield strength and ultimate strength are not statistically different significantly (unpaired t test; two-tailed $p<0.05)$. Hence, it can be deduced that in order to observe a significant detrimental effect in mechanical properties of chitosan hydrogel-based scaffolds, the porosity may have to be significantly higher than those able to be examined in this research due to sample fabrication limitations. 
Similarly, it is also possible that the mechanical strength may be improved as the porosity is further lowered. The porosity of air-dried chitosan hydrogel-based scaffolds can be further reduced by $3 \mathrm{D}$ printing scaffolds with macropores smaller than $1.5 \mathrm{~mm} \times 1.5 \mathrm{~mm}$ in dimension in the wet state. However, this hypothesis could not be tested, since, when using an extrusion nozzle with $0.42 \mathrm{~mm}$ internal diameter and $5 \% \mathrm{w} / \mathrm{v}$ chitosan hydrogel (in $2 \%$ $\mathrm{v} / \mathrm{v}$ acetic acid) as the printing ink in the 3D printer used in this research, the minimum achievable pore dimension is $1.5 \mathrm{~mm} \times 1.5 \mathrm{~mm}$ (in wet state), below this the pores collapse during the printing process.

\begin{tabular}{|c|c|c|c|}
\hline $\begin{array}{c}\text { Macropore size } \\
(\mathrm{mm})\end{array}$ & $\begin{array}{c}\text { Compressive modulus } \\
\text { (MPa) }\end{array}$ & $\begin{array}{c}\text { Yield strength } \\
\text { (MPa) }\end{array}$ & $\begin{array}{c}\text { Ultimate strength } \\
\text { (MPa) }\end{array}$ \\
\hline $0.62 \pm 0.01$ & 377.25 & 27.59 & 36.10 \\
\hline $0.99 \pm 0.13$ & 342.13 & 31.01 & 40.71 \\
\hline
\end{tabular}

Table 5.1: A representative suite of measurements obtained for one sample of each type.

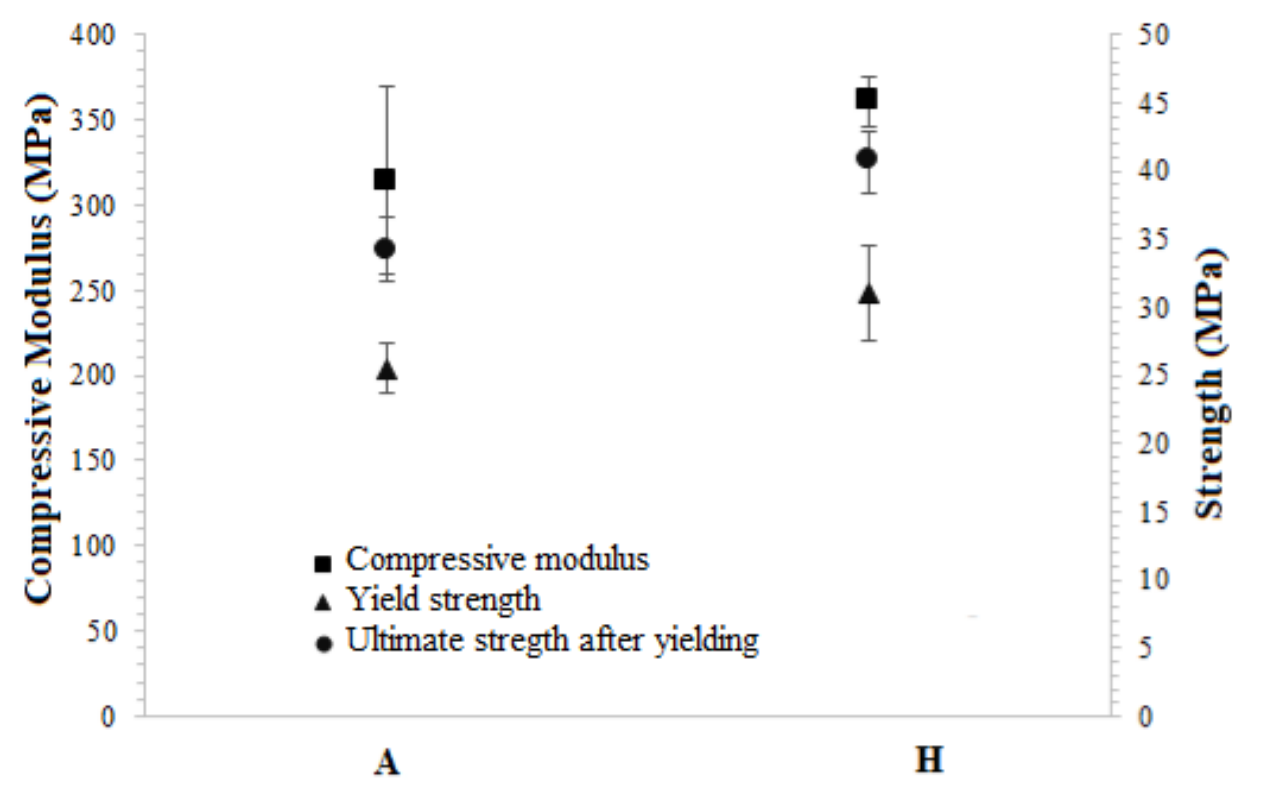
A - Chitosan hydrogel-based scaffolds with $0.62 \pm 0.01 \mathrm{~mm}$ macropores
H - Chitosan hydrogel-based scaffolds with $0.99 \pm 0.13 \mathrm{~mm}$ macropores

Figure 5.4: Variation in the compressive modulus and, the yield and ultimate strength of air-dried chitosan hydrogel-based scaffolds with different macropore dimensions. The error bars represent the standard deviation of the values obtained for five replicates of each type from the mean.

Furthermore, literature suggests that the pore size and porosity range chosen in this study is applicable for osteoblast cell proliferation and bone formation [42, 43]. Accordingly, since 
no significant variation is observed in the mechanical properties of the chitosan hydrogelbased scaffolds with the variation in porosity, only scaffolds with the lower porosity (in dried state) were chosen for further experiments.

\subsection{1b Effect of introducing minerals within chitosan hydrogel-based scaffolds}

In this study, chitosan hydrogel-based scaffolds with $1.5 \mathrm{~mm} \times 1.5 \mathrm{~mm}$ macropores (in its wet state), were mineralized via the McGrath method (alternate soaking (24 h) + Kitano (1 week)) in the presence of $2.5 \%$ w/w PAA (w.r.t dry weight of chitosan used). Composites with different percentage mineral content were fabricated by varying the concentration of the mineral precursor ion solutions used during the alternate soaking step in the McGrath method. For this, the concentration of the mineral precursor ion solutions was varied from 0.1-0.5 M. The mineralized scaffolds were then air-dried and hand-filed before mechanical testing to ensure that the area of contact with the load cell was as parallel as practically possible. Five sets of samples of each type were tested under compression. The calculated modulus, yield strength and ultimate strength are expressed as an average of the five replicates.

It is important to note that filing results in the loss of a certain amount of $\mathrm{CaCO}_{3}$ crystallites from within the 3D composites. This is because a large amount of mineralization occurs on the exterior surface of the scaffold that is directly exposed to mineralization media and this is lost during sample filing. Hence, the upper limit of the final percentage mineral content of the mineralized chitosan hydrogel-based scaffolds that are subjected to mechanical testing will be lower than the estimates obtained via TGA analysis as described in Chapter 3 (Table 3.4). Hence, to ensure accuracy in the final mineral content of the mechanically tested scaffolds, the percentage $\mathrm{CaCO}_{3}$ content of all the samples tested were analysed via TGA after mechanical testing. Table 5.2 gives the final $\mathrm{CaCO}_{3}$ content (in percentage) of mechanically tested chitosan- $\mathrm{CaCO}_{3}$ composites obtained by mineralization of chitosan hydrogel-based scaffolds via McGrath mineralization method using varying concentration of precursor ions solutions (in the presence of $2.5 \%$ w/w PAA w.r.t to the dry weight of chitosan used). 


\begin{tabular}{|c|c|}
\hline Mineralization & $\begin{array}{c}\text { \% Residual volume } \\
\text { (After mechanical testing) }\end{array}$ \\
\hline $\begin{array}{c}\text { McGrath mineralization: alternate soaking in 0.1 M } \\
\text { precursor ion solution (24 h) + Kitano (1 week) }\end{array}$ & $7.4 \pm 1.3$ \\
\hline $\begin{array}{c}\text { McGrath mineralization: alternate soaking in } 0.25 \mathrm{M} \\
\text { precursor ion solution (24 h) + Kitano (1 week) }\end{array}$ & $13.5 \pm 0.6$ \\
\hline $\begin{array}{c}\text { McGrath mineralization: alternate soaking in 0.5 M } \\
\text { precursor ion solution (24 h) + Kitano (1 week) }\end{array}$ & $30.3 \pm 2.3$ \\
\hline
\end{tabular}

Table 5.2: The percentage residual volume obtained via TGA analysis of the composites after compression testing. The analysis performed to determine the \% calcium content is the same as described in Chapter 3. As described in Chapter 3, it should be noted that the total calcium content expressed here is only an approximation to the actual amount and is hence considered as the upper limit to the total percentage mineral content.

The mineralization achieved within chitosan hydrogel-based scaffolds is very low in comparison to that of biomaterials such as nacre $(95 \%)$ or bone $(60 \%)[44,45]$. In order to compare the mechanical properties of the composites fabricated in this research to that of bone or nacre, the percentage mineral content within the samples tested (i.e. air-dried composites after filing) has to be increased. Based on the observation from Chapter 3 , this can be done by increasing the period of exposure and concentration of the precursor ions involved in the McGrath mineralization process. However, it was observed that when increasing the concentration of precursor ion solution beyond $0.5 \mathrm{M}$, the chitosan hydrogelbased scaffolds tend to swell and eventually dissolve, especially during soaking in the sodium hydrogen carbonate solution, within an hour of exposure. Hence, the mineralization protocol used in this research will require further modification in order to develop composites with higher calcium carbonate content and thus be comparable to that of nacre.

Figure 5.5 depicts the variation in the compressive modulus, yield strength and the ultimate strength of chitosan hydrogel-based scaffolds and chitosan- $\mathrm{CaCO}_{3}$ composites with varying percentage calcium carbonate content as fabricated via the McGrath method by altering the concentration of the mineral precursor ion solutions used during the alternating soaking step.

The data suggest that the mechanical strength and modulus of the scaffolds increases initially with the presence of very small quantities of $\mathrm{CaCO}_{3}$ crystallites $(7.4 \pm 1.3 \%)$ but 
then decreases with further increase in the percentage mineral content. The yield strength showed a similar trend although the initial increase in the strength is very small. Statistically significant variation (one-way ANOVA, $\mathrm{n}=5, p<0.05$ ) was however observed only in the case of the yield strength values obtained for (1) pure chitosan based samples and composites fabricated using 0.5 M precursor ion solution (refer to legends in Figure 5.5, A versus $\mathrm{D}$; adjusted $\mathrm{p}$ value $=0.01)$, (2) composites with the highest and lowest mineral content $(B$ versus $D$; adjusted $p$ value $=0.04)$ and in the ultimate strength values obtained for pure hydrogel-based scaffolds and composites fabricated using $0.5 \mathrm{M}$ precursor ion solution (A versus D; adjusted p value $=0.04)$.

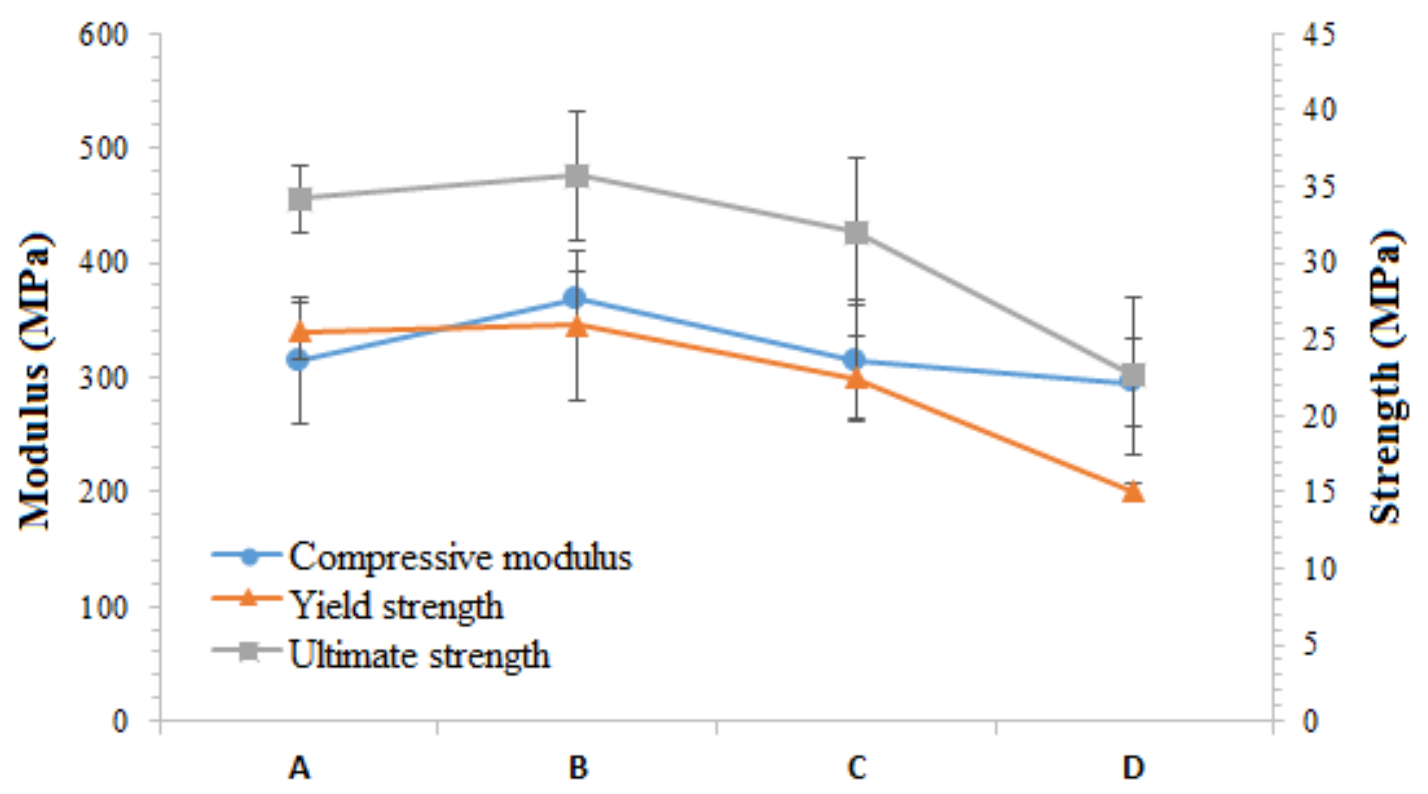
A- Chitosan hydrogel-based scaffold
B- McGrath mineralized chitosan hydrogel-based scaffold
B- (0.1 M alternate soaking $(24 \mathrm{~h})+\mathrm{Kitano}(1$ week $))$
C - McGrath mineralized chitosan hydrogel-based scaffold
$(0.25 \mathrm{M}$ alternate soaking $(24 \mathrm{~h})+$ Kitano (1 week)
D - McGrath mineralized chitosan hydrogel-based scaffold ( $0.5 \mathrm{M}$ alternate soaking $(24 \mathrm{~h})+$ Kitano (1 week)

Figure 5.5: Variation in the compressive modulus, yield strength and ultimate strength of chitosan-CaCO $\mathrm{C}_{3}$ composites with different percentage calcium carbonate content fabricated via the McGrath method in comparison to non-mineralized chitosan hydrogel-based scaffolds. All samples tested are in the dried state and the error bars represent the standard deviation from the mean of the values calculated for five replicates of each type of composite tested. Lines are to guide the eye only. 
The highest compressive modulus, yield strength and ultimate strength of $0.37 \mathrm{GPa}, 25.5$ $\mathrm{MPa}$ and $31 \mathrm{MPa}$ respectively corresponds to composites fabricated via the McGrath mineralization method using $0.1 \mathrm{M}$ mineral precursor ion solutions (24 h alternate soaking +7 days Kitano treatment) in the presence of $2.5 \%$ PAA w/w w.r.t dry (weight of chitosan used). Literature suggests that this is comparable to that of medium density trabecular bone [46, 47]. TGA analysis of these scaffolds after mechanical testing revealed that their percentage mineral content was as low as $7.4 \pm 1.3 \%$ calcium carbonate. Although the percentage loading may seem very small, certain studies have reported very low percentage filler loading (0.1-0.3 wt \%) to have enhanced the tensile modulus by up to $200 \%$ [11].

The observed trend shown in Figure 5.5 is very similar to the studies reported in literature suggesting an increase in the impact strength, tensile modulus, bending strength and hardness up to a limit followed by a decrease due to clustering of the filler particles with increases in the percentage loading [48-52]. Hence, the observation of a decreasing trend in the mechanical properties of the composite scaffolds with increasing $\mathrm{CaCO}_{3}$ content could be a sign of aggregation or clustering. The observations described in Chapter 3 show that chitosan hydrogel-based scaffolds mineralized via the McGrath method in the presence of PAA resulted in mineral growth occurring in association with the hydrogel matrix in the form of oriented aggregation of nano-sized calcium carbonate crystallites (Section 3.3.2), thereby potentially supporting this hypothesis.

Levengood and Zhang have reported a compilation of mechanical properties of porous chitosan hydrogel-based composites fabricated via different methods [53]. Upon comparison with these, it can be deduced that the mechanical properties of chitosan hydrogel-based scaffolds and composites developed in this research are higher (an order of magnitude higher in the case of non-mineralized structures and twice in the case of composites) than those fabricated with comparable scaffold porosity, concentration of chitosan used and material testing conditions. The air-drying process combined with the scaffold architecture could be contributing factors for this enhancement in the case of nonmineralized chitosan hydrogel-based scaffolds, whereas in the case of the composites developed via the McGrath method in the presence of PAA, the reason for the higher mechanical strength could be the enhanced interaction between the organic matrix and the mineral component resulting from the aggregation of nano-sized $\mathrm{CaCO}_{3}$ crystallites in association with the polymer matrix (as explained in Chapter 3). The higher surface-tovolume ratio of the nanoparticles interacting with the organic matrix may have resulted in better stress distribution under compression $[54,55]$. 
However, the observed decreasing trend in the mechanical properties of the composites fabricated via the McGrath method with increasing percentage $\mathrm{CaCO}_{3}$ content despite the enhanced nanoparticles-matrix interaction shows that the size and morphology of the resultant polymer-nanoparticle aggregates could also be contributing factors to the overall mechanical strength of the composites. Although in this research aggregation/clustering of nano-sized $\mathrm{CaCO}_{3}$ crystallites is observed in all the composites when using the McGrath mineralization method in the presence of $2.5 \%$ w/w PAA ( $24 \mathrm{~h}$ alternate soaking each + Kitano soaking (1 week)), the size of the resultant micron-sized crystallites (in aggregated form) increased with the concentration of the mineralization solution (Chapter 3, Section 3.3.4). The particle size varied from $11.5 \pm 2.6 \mu \mathrm{m}$ to $30.0 \pm 4.7 \mu \mathrm{m}$ as the concentration of the precursor ion solutions was increased from $0.1 \mathrm{M}-0.5 \mathrm{M}$ respectively. Data from the mechanical tests showed that the compressive moduli and yield strength, in the case of the former, is $0.37 \pm 0.02 \mathrm{GPa}, 25 \pm 5 \mathrm{MPa}$ and that of the latter is $0.29 \mathrm{GPa} \pm 0.01,15 \pm 2 \mathrm{MPa}$ respectively. When correlating these, it can be suggested that along with the increasing mineral content, the increasing size of the resultant mineral phase may have contributed to the observed decrease in the mechanical properties of composites fabricated using high concentration precursor ion solutions via the McGrath method. Hence, it is suggested that chitosan- $\mathrm{CaCO}_{3}$ composites with a lower percentage of smaller nanoparticle-polymer aggregates could have a higher yield and ultimate strength.

The large variations (represented by standard deviation error) in the modulus and yield strength values across samples fabricated using the same mineralization solutions and (thus the statistical analysis showing that the observed variations are non-significant) could be attributed to the non-uniform/random distribution of $\mathrm{CaCO}_{3}$ crystallite within the scaffolds due to the nature of the mineralization process. Although the McGrath mineralization method (in presence of $2.5 \%$ PAA w.r.t dry weight of chitosan used) initiates a matrix dependent crystal nucleation, the extent of mineralization in the interiors of the scaffold is a diffusion dependent process and does not offer precise control over the nucleation site within the hydrogel scaffold for $\mathrm{CaCO}_{3}$ nuclei and hence results in a random distribution of crystallites (this observation is based on analysis of SEM images of the mineralization occurring in between the printed layers and the regions of the 3D scaffold that are in direct contact with the mineralization media, data not shown here). Other contributing factors could be the moisture content (based on TGA analysis-data not shown), variation in the degree of deacetylation across the chitosan polymer chain and minor structural differences arising as a result of the semi-automated $3 \mathrm{D}$ printing process and the hand filing process 
during sample preparation. Nonetheless, based on the data obtained it can be concluded that mineralization of chitosan hydrogel-based scaffolds affects its mechanical strength and that very high mineralization has detrimental effects on the same.

\subsection{1c Effect of the morphology of $\mathrm{CaCO}_{3}$ crystallites within chitosan- $\mathrm{CaCO}_{3}$ composites}

The previous study suggests that apart from the mineral content, the morphology and size of the resultant crystallites may affect the mechanical strength of composites. Hence, a study was performed to analyse the validity of the hypothesis that composites with a lower percentage of smaller nanoparticle-polymer aggregates in the form of pancake-like structures could have better mechanical properties, in the case of the chitosan- $\mathrm{CaCO}_{3}$ composites fabricated via the McGrath method.

In this study, chitosan hydrogel-based scaffolds with $1.5 \mathrm{~mm} \times 1.5 \mathrm{~mm}$ macropores (in its wet state), were mineralized via the McGrath method following a modified protocol such that the morphology of the resultant $\mathrm{CaCO}_{3}$ crystallites within the final composites best replicates the pancake-like structure of nacre in its early stages of development. Accordingly, based on the observations from Chapter 3, chitosan hydrogel-based scaffolds were mineralized by alternatively soaking in $0.25 \mathrm{M}$ mineral precursor ion solutions $(1 \mathrm{~h}$ each) + Kitano soaking (3 days)) or via alternately soaking in $0.1 \mathrm{M}$ mineral precursor ion solution $(2 \mathrm{~h}$ each $)+$ Kitano soaking (4 days) ) both in the presence of $2.5 \%$ w/w PAA (w.r.t dry weight of chitosan used). The mineralized scaffolds were then air-dried and further hand-filed before mechanical testing to ensure that the area of contact with the load cell is as parallel as practically possible. Three sets of samples of each type were tested under compression in the static mode. The calculated modulus, yield strength and ultimate strength are expressed as an average of the three samples.

The data obtained in each case is compared with composites fabricated via the McGrath method using precursor ions solutions of the same concentrations $(0.1 \mathrm{M}$ or.25 $\mathrm{M})$ but different soaking periods such that the resultant mineral phase has predominantly larger micron-sized crystallites. Table 5.3 describes the mineralization method used; the size and morphology of the resultant calcium carbonate crystallites within the composite; and resultant mechanical properties of the composites thus fabricated. 


\begin{tabular}{|c|c|c|c|c|}
\hline Mineralization method & $\begin{array}{l}\text { Compressive } \\
\text { modulus } \\
(\mathrm{MPa})\end{array}$ & $\begin{array}{c}\text { Yield } \\
\text { strength } \\
\text { (MPa) }\end{array}$ & $\begin{array}{l}\text { Ultimate } \\
\text { strength } \\
\text { (MPa) }\end{array}$ & $\begin{array}{l}\text { Morphology } \\
\text { and size of } \\
\text { particles }\end{array}$ \\
\hline $\begin{array}{l}\text { McGrath mineralization: } \\
\text { alternate soaking in } 0.1 \\
\text { M precursor ion solution } \\
(24 \mathrm{~h})+\text { Kitano (1 week) }\end{array}$ & $368 \pm 24$ & $26 \pm 5$ & $36 \pm 4$ & $\begin{array}{c}\text { Laterally } \\
\text { growing } \\
\text { aggregates of } \\
\text { nanoparticles } \\
(27 \pm 9 \mu \mathrm{m})\end{array}$ \\
\hline $\begin{array}{l}\text { McGrath mineralization: } \\
\text { alternate soaking in } 0.1 \\
\text { M precursor ion solution } \\
(2 \mathrm{~h})+\text { Kitano (4 days) }\end{array}$ & $201 \pm 27$ & $17 \pm 5$ & $21 \pm 4$ & $\begin{array}{l}\text { Pancake-like } \\
(19 \pm 4 \mu \mathrm{m})\end{array}$ \\
\hline $\begin{array}{l}\text { McGrath mineralization: } \\
\text { alternate soaking in } 0.25 \\
\text { M precursor ion solution } \\
(24 \mathrm{~h})+\text { Kitano ( } 1 \text { week) }\end{array}$ & $301 \pm 49$ & $23 \pm 3$ & $32 \pm 5$ & $\begin{array}{l}\text { Irregular and } \\
\text { some pancake- } \\
\text { like aggregates } \\
\text { of diameter } \\
27 \pm 6 \mu \mathrm{m}\end{array}$ \\
\hline $\begin{array}{l}\text { McGrath mineralization: } \\
\text { alternate soaking in } 0.25 \\
\text { M precursor ion solution } \\
(1 \mathrm{~h})+\text { Kitano ( } 3 \text { days })\end{array}$ & $318 \pm 29$ & $18 \pm 4$ & $30 \pm 6$ & $\begin{array}{c}\text { Pancake-like } \\
(26 \pm 6 \mu \mathrm{m})\end{array}$ \\
\hline
\end{tabular}

Table 5.3: Variation in the compressive modulus, yield and the ultimate strengths of chitosan- $\mathrm{CaCO}_{3}$ composites fabricated via McGrath mineralization of chitosan hydrogelbased scaffolds by alternately soaking in $0.1 \mathrm{M}$ or $0.25 \mathrm{M}$ mineral precursor ions solution and soaking in Kitano solution, for varying time periods. Also given is the resultant morphology and the size of the calcium carbonate crystallites formed in between the layers of the scaffold (Refer to Chapter 3, Section 3.3.5 for corresponding SEM images).

The data indicates (Table 5.3) that, when using 0.1 M mineral precursor ion solutions, the compressive modulus, the yield strength and the ultimate strength of composites fabricated with predominantly smaller pancake-like crystallites ( $2 \mathrm{~h}$ alternate soaking +4 days Kitano) was significantly (statistically significant; $\mathrm{n}=3$ unpaired t-test, 2 tailed $p<0.05$ ) lower than 
the those obtained for composites with predominantly larger laterally growing aggregates of nanoparticles ( $24 \mathrm{~h}$ alternate soaking +7 days Kitano). TGA analysis revealed that the total mineral content of the former $(2.4 \pm 1.1 \%)$ is about a fourth of the latter $(7.4 \pm 1.3 \%)$. The data also suggests that the strength and modulus of the former are even lower than nonmineralized chitosan hydrogel-based scaffolds (Figure 5.5). Hence, it cannot be suggested for this data that, the hypothesis that composites with a lower percentage of smaller nanoparticle-polymer aggregates in the form of pancake-like structures may have better mechanical properties is true in the case of composites fabricated with pancake-like aggregates via the McGrath mineralization method using $0.1 \mathrm{M}$ precursor ion solutions.

In the case of composites fabricated using $0.25 \mathrm{M}$ mineral precursor ion solution, the compressive modulus, the yield strength and the ultimate strength of composites with predominantly pancake-like crystallites ( $1 \mathrm{~h}$ alternate soaking +3 days Kitano) were comparable to those with predominately larger non-pancake-like nanoparticle aggregates ( $24 \mathrm{~h}$ alternate soaking +7 days Kitano). TGA analysis of these composites after mechanical testing showed that the final percentage $\mathrm{CaCO}_{3}$ content of the mechanically tested samples in both the cases were comparable $(\sim 14 \%)$. In this case, the hypothesis that composites with a lower percentage of smaller nanoparticle-polymer aggregates in the form of pancake-like structures may have better mechanical properties, could be true.

The mechanical properties of chitosan- $\mathrm{CaCO}_{3}$ composites with crystallite morphologies in the form of pancake-like or non-pancake-like aggregates of nanosized $\mathrm{CaCO}_{3}$ crystallites were within the range of those of cancellous bone at different regions of the skeletal system [56-58]. Studies have also shown that lamellar/platelet-like inclusion improve the mechanical properties of polymeric systems owing to their higher aspect ratio and that the same is true for particles with a lower aspect ratio that are well bound to the polymer matrix [59]. In the case of composites fabricated in this study, the filler particles are flat pancakelike structures (high aspect ratio) that are formed of nanoparticles of calcium carbonate intricately associated with the polymer matrix. However, it is inconclusive at this stage whether the lateral growth of calcium carbonate crystallites in the form of pancake-like structure is advantageous in terms of enhancing the mechanical strength of the chitosan$\mathrm{CaCO}_{3}$ composites and more investigations would be required.

Nonetheless, based on the observation from the compression testing of chitosan- $\mathrm{CaCO}_{3}$ composites, it is clear that the McGrath mineralization of the hydrogel scaffolds (in wet state) in the presence of PAA, resulting in the aggregation of nano-sized $\mathrm{CaCO}_{3}$ in 
association with the chitosan hydrogel matrix, significantly enhances the mechanical strength of air-dried chitosan- $\mathrm{CaCO}_{3}$ composites in comparison to chitosan-mineral composites reported in literature with comparable properties (scaffold porosity, concentration of chitosan used and testing condition used) [53]. TGA analysis shows that this is true when the percentage $\mathrm{CaCO}_{3}$ is as low as $7 \%$. Also, it can be suggested that a significant decrease in the mechanical properties may be observed when the total mineral content in the composites is more than $40 \%$, in this case, the size of the resultant crystallites may also be considered as a contributing element.

As the pancake-like morphology did not seemingly enhance the mechanical properties of the composites in the static mode, these were not further studied under dynamic loading conditions.

\subsubsection{Dynamic loading}

Dynamic testing under in vivo-like stress loading conditions is the next step of mechanical characterization for composites that displayed appreciable mechanical strength when compressed under static loading conditions. Accordingly, in this study, chitosan hydrogelbased scaffolds with $1.5 \mathrm{~mm} \times 1.5 \mathrm{~mm}$ macropores (in its wet state), were mineralized via the McGrath method (alternate soaking (24 h) + Kitano (1 week)) in the presence of $2.5 \%$ w/w PAA (w.r.t dry weight of chitosan used). Composites with different percentage mineral content were fabricated by varying the concentration of the mineral precursor ion solutions used during the McGrath method. The concentration of the mineral precursor ion solutions was varied from $0.1 \mathrm{M}-0.5 \mathrm{M}$. The mineralized scaffolds were then air-dried and hand-filed before mechanical testing to ensure that the area of contact with the load cell is as parallel as practically possible. Five replicates of each type of sample were tested under compression under a dynamic oscillatory loading, with the loading frequency varying from $1 \mathrm{~Hz}$ to 15 $\mathrm{Hz}$ using an SDTA $861^{\mathrm{e}}$ Mettler Toledo instrument equipped with a $40 \mathrm{~N}$ load cell. The data obtained was processed using the inbuilt software and displayed in the form of storage, loss and complex modulus.

When considering biopolymer-based composites as prospective bone graft materials it is important to consider the viscoelastic properties of the polymer components along with the contribution of the fillers $\left(\mathrm{CaCO}_{3}\right)$. This property cannot be studied in detail using static stress loading tests, dynamic oscillatory stress loading however can give better information on the contribution of the organic component of the composite. Also, in order to study the 
long-term performance of composite materials in an in vivo-like environment, it is important to study their behaviour under dynamic and cyclic loading conditions [60].

Dynamic mechanical analysis was performed within a loading force ranging from $0-12 \mathrm{~N}$ (assuming that the strain induced is within the elastic limit when compressed by a $40 \mathrm{~N}$ load cell as in the DMTA machine, based on the data obtained from the static loading tests performed on a different instrument described in pervious sections using a $5 \mathrm{kN}$ load cell). The compressive force was applied under a varying loading frequency of between 1 - 15 Hz. This loading frequency is well within physiologically relevant loading frequencies experienced by the human skeletal system while performing daily activities such as walking, running and exercising $(1-50 \mathrm{~Hz})[61,62]$.

Figure 5.6 shows the variation in the storage, loss and complex moduli (average of those obtained for five replicates of each type of sample tested) of chitosan- $\mathrm{CaCO}_{3}$ composites with increasing $\mathrm{CaCO}_{3}$ content. The general trend observed in the storage modulus and the complex modulus irrespective of the mineral content, can be divided into three sections (marked by dashed lines in Figure 5.6) a) lower frequencies $(1-9 \mathrm{~Hz})$ : where the storage modulus is essentially constant; b) 9 - $13 \mathrm{~Hz}$ : increase in the modulus; and c) beyond $13 \mathrm{~Hz}$ : where the modulus is constant, the value being much higher than in region "a" and the corresponding loss modulus at this frequency. The loss modulus was essentially constant across all frequencies irrespective of the sample composition.

A marked variation is not observed in the trend followed by the storage and complex moduli in regions "a" and " $b$ " for the chitosan hydrogel-based with variation in the percentage $\mathrm{CaCO}_{3}$ content. However, a distinct decreasing trend is observed in region "c", wherein, the storage modulus decreased with increasing percentage $\mathrm{CaCO}_{3}$ content in the scaffolds. The composites fabricated via McGrath mineralization of chitosan hydrogel-based scaffolds in the presence of $0.1 \mathrm{M}$ precursor ion solutions (24 h alternate soaking +1 week soaking in Kitano solution), showed the highest storage and complex modulus within this frequency range. Compared to non-mineralized scaffolds, the storage modulus at frequencies beyond $13 \mathrm{~Hz}$ was higher for all composite samples. 

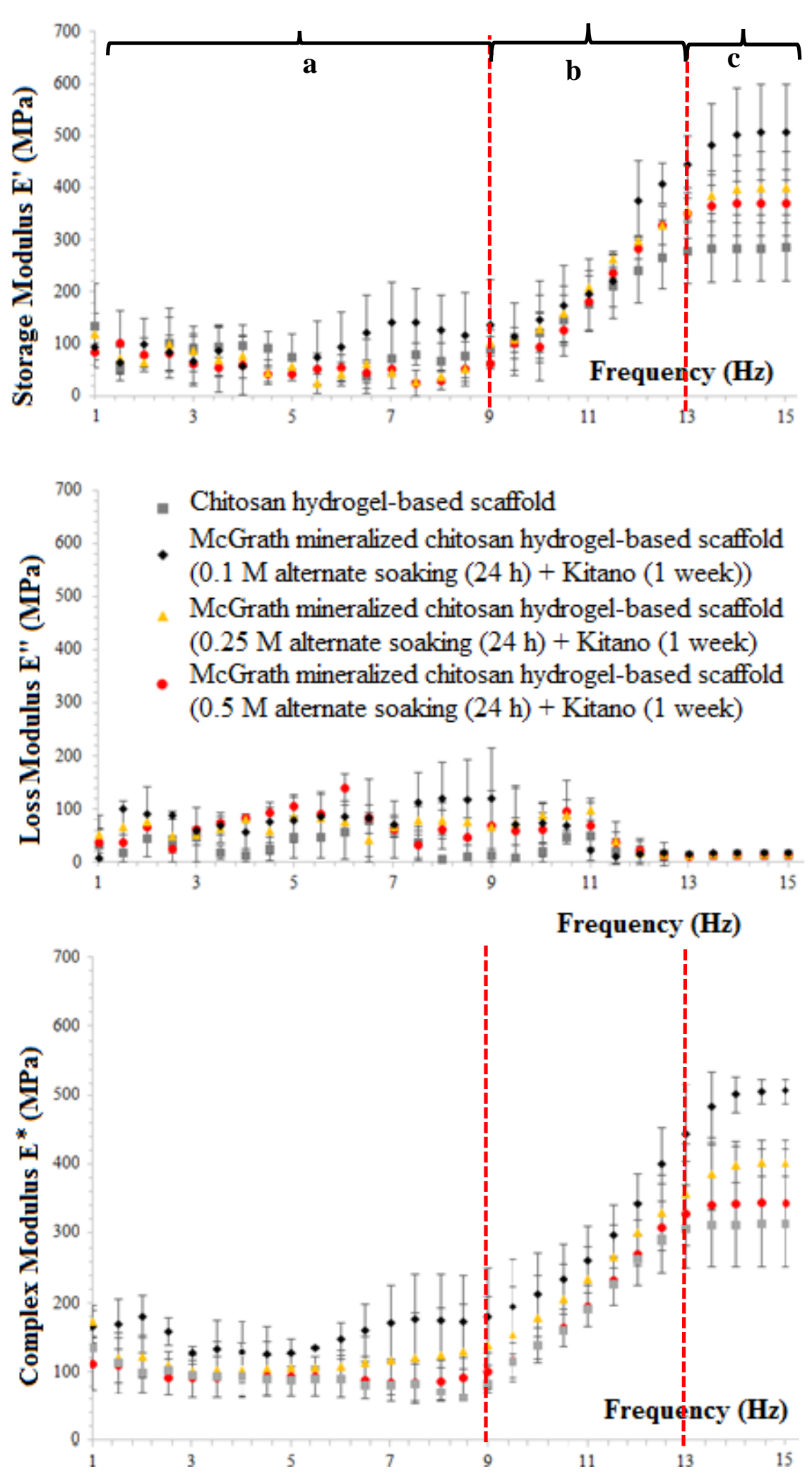

Figure 5.6: Variation in the dynamic storage, loss and complex moduli of chitosan hydrogel-based scaffolds with increasing $\mathrm{CaCO}_{3}$ content introduced via the McGrath mineralization method. Five replicates of each type of sample were tested and the error bars represent the standard deviation from the mean of the values obtained in each case. The dotted lines distinguish the regions ' $a$ ', ' $b$ ' and 'c' referred to in Section 5.3.2. 
There are few studies reporting the viscoelastic behaviour of biopolymer-mineral composites under varying dynamic loading frequency, in non-hydrated conditions. However, a number of studies report a similar behaviour in synthetic bone tissue engineering scaffolds and natural bone in hydrate state. For example, Wands et al. reported a similar trend with the increasing mass fraction of hydroxyapatite in calcium alginate hydrogels (in hydrated conditions), wherein the storage modulus was found to increase and further attain a constant value with increasing dynamic loading frequency. This was correlated to glass transition-like behaviour wherein a material transitions from a soft to a glassy state with increasing temperature [63]. Similarly, Sadeghi et al. and Fulcher et al. studying the viscoelastic properties of bovine articular cartilage attached to subchondral bone (stored in ringer's solution) reported a similar behaviour under loading frequencies below and far above physiologically relevant values [62,64]. They suggest that the increase in the storage modulus relative to minor variations in the loss modulus with respect to the loading frequency suggest that the excess energy is stored within the composite as the loading frequency increases.

In the above examples, the presence of fluid (water) within the test sample will have a large contribution to the resultant trend observed as water tends to behave as an incompressible solid at higher loading frequencies, thus resulting in proportionally higher storage modulus values at these loading frequencies. Although the results from these studies are not comparable with the data obtained here owing to the variation in the testing conditions, the similarity in the trend with those reported for hydrated cartilage samples (composed of only biopolymers, no mineral) suggests that the viscoelastic properties of chitosan hydrogel (in dry condition) is a prominent contributing factor to the dynamic response of our composites, as in this case no fluids are involved that may contribute to the viscoelastic response behaviour.

In order to ascertain the stability of chitosan hydrogel-based scaffolds under high frequency stress loading, in terms of failure induced within the scaffold structure (microcracks that may not be identified by physical observation), all of the above samples that were tested via DMA under varying frequencies were then subject to uniaxial compression testing in static mode until failure. Uniaxial testing was performed in static mode using the same testing parameters ( $5 \mathrm{kN}$ load cell, $0.1 \mathrm{~mm} / \mathrm{s}$ crosshead speed, and $2 \mathrm{~N}$ preload) and instrument as described in section 5.3.1. The compressive modulus and yield strength calculated from the stress-strain curve thus obtained are compared with those reported in section 3.5.1b i.e., unstressed chitosan hydrogel-based scaffolds or composites. Given the standardization of the 
3D printing, fabrication parameters and mineralization process, any variations in the values obtained will suggest failure in the form of microcracks as a result of high frequency loading. Figure 5.7 shows the variation in the compressive modulus and the yield strength of samples subjected to frequency scan and un-stressed samples.

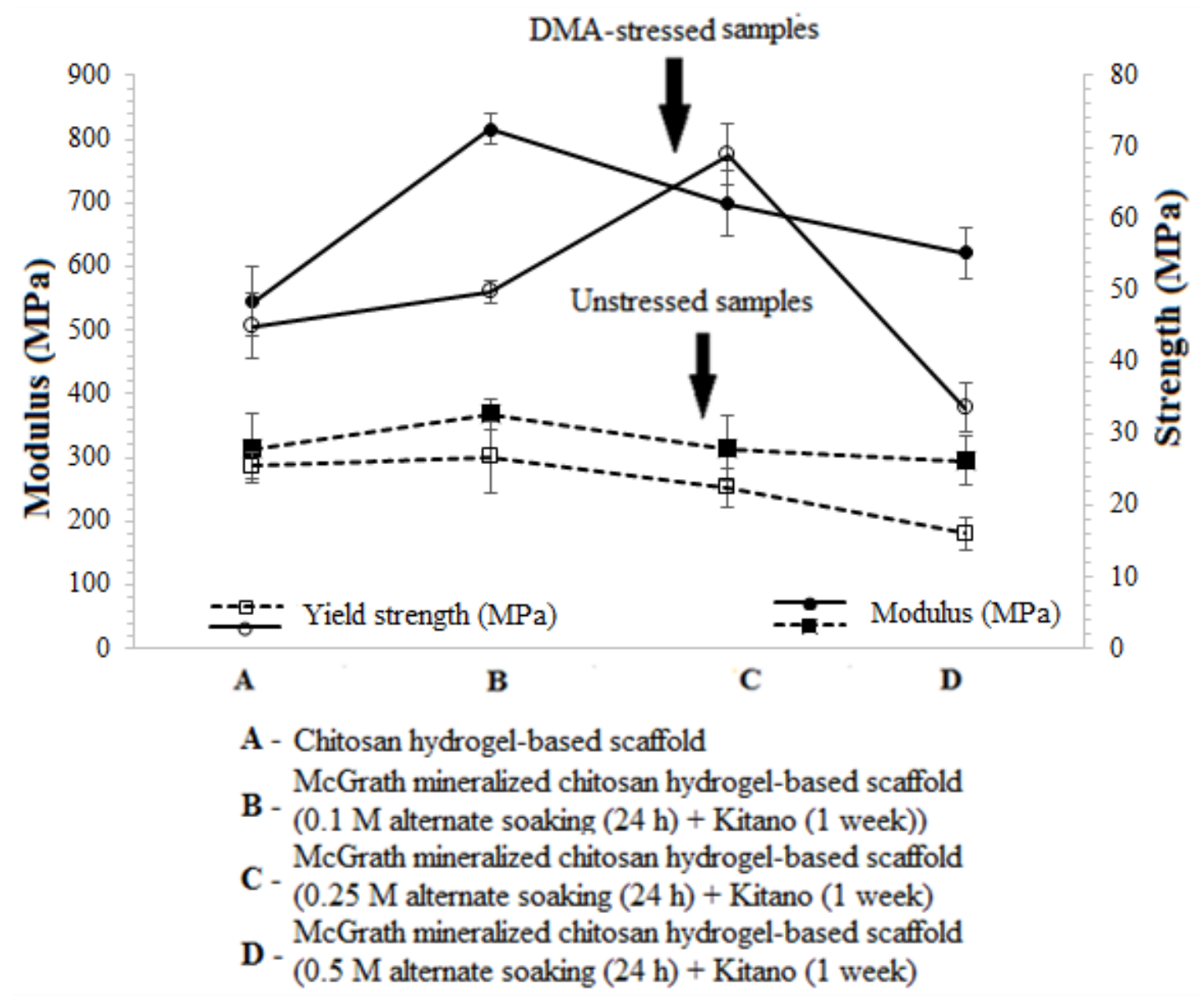

Figure 5.7 Variation in the compressive modulus and the yield strength of samples subjected to DMA-frequency scans in comparison to those of previously un-stressed samples. The data in dashed lines represent the behaviour of un-stressed samples and solid lines correspond to samples previously subjected to dynamic frequency scans using a DMA instrument. The lines themselves are to aid in comparison only.

Although the samples subjected to high loading frequency depicted a similar trend to that of the un-stressed samples i.e. a decrease mechanical strength with the increasing percentage $\mathrm{CaCO}_{3}$ content, the calculated compressive modulus and yield strength in the case of the former were almost double that of the corresponding un-stressed samples. Hence, it is deduced that dynamic stress loading at increasing loading frequencies can result in irreversible deformation within the $3 \mathrm{D}$ chitosan hydrogel-based structures and composites irrespective of the mineral content. However, it is important to note that the deformations 
induced do not lead to complete fracture of these 3D structures under the given stress loading conditions.

The observation from all the above mechanical tests suggest that air-dried 3D printed chitosan hydrogel-based scaffolds (non-mineralized as well as the composites) have a much higher resistance to ultimate fracture (based on the yield and the ultimate strength values) of the samples compared to other reported studies of similar biopolymer-based composites. The values obtained in our case are within the same order as that of trabecular bone despite the porous architecture. Also, the stability of the scaffolds under dynamic stress loading within physiologically relevant loading frequencies (similar to the cyclic stress loading during brisk walking or exercising) suggest that such structures show considerable potential towards application as bone graft materials for fracture healing. Nonetheless, from an application point of view as in vivo bone grafts, it is important to characterise the composites generated in this research in a physiologically relevant environment, i.e. in a hydrated state. This is beyond the scope of this thesis and will be studied in future.

\subsubsection{Indentation hardness}

Hardness testing (nanoindentation) was performed to identify the nanoscale hardness at different regions for those 3D samples (composites as well as non-mineralized scaffolds) that showed the best mechanical characteristics on a macroscale as observed during the compression testing performed under uniaxial static and dynamic stress loading.

For this study chitosan hydrogel-based scaffolds with $1.5 \mathrm{~mm} \times 1.5 \mathrm{~mm}$ macropores (in its wet state), were mineralized via the McGrath method (alternate soaking (24 h each) + Kitano (1 week)) in the presence of $2.5 \%$ w/w PAA (w.r.t dry weight of chitosan used) and composites with different percentage mineral content were fabricated by varying the concentration of the mineral precursor ion solutions used during the McGrath method. The concentration of the mineral precursor ion solutions was varied from $0.1 \mathrm{M}-0.5 \mathrm{M}$. The mineralized scaffolds were then air-dried, hand-filed and polished using silicon carbide polisher before indentation testing to ensure that the surface to be indented is smooth. One sample of each of the variants fabricated was used in this study and multiple indentations were performed at different regions of the sample to analyse the hardness at each of those regions. The total hardness is expressed as an average of the values obtained for more than 15 different indentations. The hardness values depicted here were obtained through a computerised data acquisition software inbuilt in the instrument. 
Table 5.4 summarises the variation in the nanoindentation hardness values for different regions within the samples, namely the struts and the nodes (regions were struts overlap) within the lattices and the regions forming the hollow cylinder. Statistical analysis (one-way ANOVA) showed that within individual sample types, there is no significant variation in hardness values obtained at various regions of the sample in all cases except in the case of composites fabricated via mineralization of chitosan hydrogel-based scaffolds using the McGrath method in the presence of $0.25 \mathrm{M}$ precursor ion solution ( $\mathrm{n}=5$, adjusted $p<0.0001)$. The deviation from the trend may be due to the limited mineral growth on the indented location owing to the randomness in the mineral nucleation within the $3 \mathrm{D}$ hydrogel-based scaffolds.

\begin{tabular}{|c|c|c|c|}
\hline \multirow{2}{*}{ Sample } & \multicolumn{2}{c|}{ Hardness (GPa) } \\
\cline { 2 - 4 } & Hollow & Struts & Node \\
cylinder & & \\
(no mineral) & $0.20 \pm 0.04$ & $0.15 \pm 0.05$ & $0.17 \pm 0.02$ \\
\hline $\begin{array}{c}\text { Chitosan hydrogel-based scaffold } \\
\text { McGrath mineralization: alternate } \\
\text { soaking in 0.1 M precursor ion solution } \\
(24 \text { h) + Kitano (1 week) }\end{array}$ & $0.30 \pm 0.09$ & $0.23 \pm 0.11$ & $0.23 \pm 0.10$ \\
\hline $\begin{array}{c}\text { McGrath mineralization: alternate } \\
\text { soaking in 0.25 M precursor ion } \\
\text { solution (24 h) + Kitano (1 week) }\end{array}$ & $0.18 \pm 0.02$ & $0.36 \pm 0.05$ & $0.38 \pm 0.05$ \\
\hline $\begin{array}{c}\text { McGrath mineralization: alternate } \\
\text { soaking in 0.5 M precursor ion solution } \\
(24 \text { h) + Kitano (1 week) }\end{array}$ & $0.26 \pm 0.06$ & $0.31 \pm 0.11$ & $0.30 \pm 0.11$ \\
\hline
\end{tabular}

Table 5.4: Hardness values for different regions within a sample. The data given here are the average of the values obtained from more than five different indentations performed at each of the respective regions of a single sample of each type.

The combined average hardness values of non-mineralized scaffolds and that of composites with varying percentage mineral content are shown in Figure 5.8.

The observed general trend is that hardness values $(H)$ and the indentation modulus $\left(E_{r}\right)$ of chitosan hydrogel-based structures increased following mineralization. Statistical analysis ( $\mathrm{n}=5$, two-way ANOVA) showed that the initial increase in the hardness of the composite upon mineralization using a low concentration of precursor ion was not significant (A versus 
$\mathrm{B}$; refer to legends in Fig 5.8). However, there is a significant difference (increasing trend) between the combined average hardness value of non-mineralized samples and composites developed using: (1) $0.25 \mathrm{M}$ precursor ion solutions (increasing trend; A versus C; adjusted $\mathrm{p}$ value $=0.0016$ ); and (2) $0.5 \mathrm{M}$ precursor ion solutions (increasing trend; A versus D; adjusted $\mathrm{p}$ value $=0.02$ ). Also, no statistically significant variation was observed in the combined hardness values between composites with increasing mineral content (B-C-D), which may be due to the anomalous values obtained for composites obtained via McGrath mineralization using $0.25 \mathrm{M}$ precursor ion solutions as described earlier.

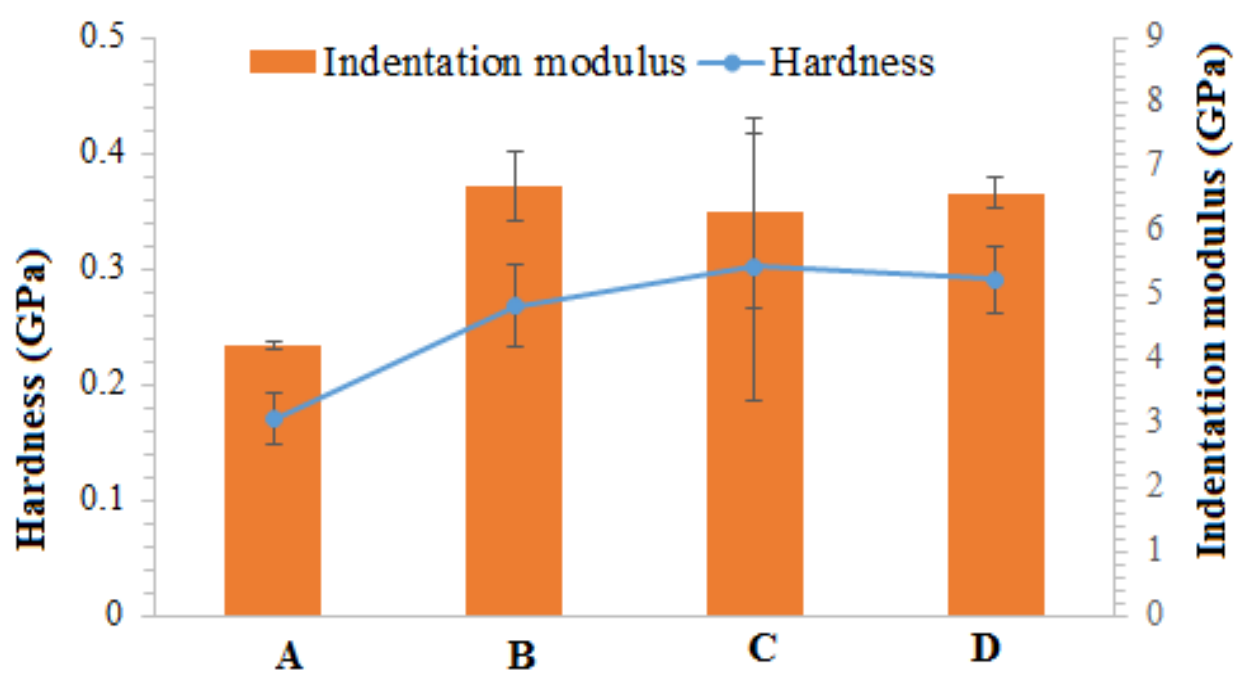
A- Chitosan hydogel-based scaffold
B- McGrath mineralized chitosan hydrogel-based scaffold (0.1 M alternate soaking $(24 \mathrm{~h})+\mathrm{Kitano}$ (1 week))
C. McGrath mineralized chitosan hydrogel-based scaffold $(0.25 \mathrm{M}$ alternate soaking $(24 \mathrm{~h})+\mathrm{Kitano}$ (1 week))
D - McGrath mineralized chitosan hydrogel-based scaffold (0.5 M alternate soaking $(24 \mathrm{~h})+\mathrm{Kitano}(1$ week $))$

Figure 5.8 Variation in the average combined hardness (average of the hardness obtained for different regions of the scaffold) and indentation elastic modulus (reduced modulus) of a non-mineralized scaffold and composites with varying percentage mineral content fabricated by the mineralization of chitosan hydrogel-based scaffolds via the McGrath method. The error bars represent the standard deviation of the values obtained from the mean, for 15 different indentations performed at various regions of the scaffolds (the nodes, struts and hollow cylindrical region).

Similarly, composites depicted a higher combined average indentation modulus compared to pure chitosan hydrogel-based samples. A significant increase was observed between nonmineralized samples and composites developed using: (1) $0.5 \mathrm{M}$ precursor ion solution (A 
versus $\mathrm{D}$; adjusted $\mathrm{p}$ value $=0.03$ ), and a decreasing trend was observed between the samples with lowest and highest minerals content (B versus $D$; adjusted $\mathrm{p}$ value $=0.03$ ). The data obtained are of the same order as that of trabecular bone of the vertebral column and mandibular bone cementum wherein the indentations were performed under comparable experimental parameters (loading force, hold time and indentation depth) [65-67]. 


\subsection{Conclusion}

The mechanical properties of 3D printed chitosan hydrogel-based scaffolds and composites fabricated by mineralization of the chitosan hydrogel-based scaffolds via the McGrath method, under uniaxial compressive loading in static and dynamic mode; and nanoindentation were studied. The effect of various parameters such the porosity of the 3D scaffold, percentage calcium carbonate content, the morphology, and size of the crystallites within the hydrogel matrix were analysed for air-dried scaffolds.

Under static loading conditions, the following were observed:

(a) air-dried 3D porous chitosan hydrogel-based scaffolds and composites depicted higher mechanical strength and compressive modulus in comparison to other porous chitosanbased scaffolds with similar structural properties reported in literature. When the porosity of the dried chitosan hydrogel-based scaffolds is varied from $51.6 \pm 1.3 \%$ to $58.8 \pm 1.2 \%$ by increasing the printed macropore dimensions from $0.62 \pm 0.01 \mathrm{~mm}$ to $0.99 \pm 0.13 \mathrm{~mm}$, no significant variation was observed in the mechanical properties of the resultant scaffolds. However, considering that the porosity in both cases is within the range suitable for osteoblast cell proliferation and bone formation, scaffolds with lower porosity were chosen for further studies.

(b) calcium carbonate mineralization of chitosan hydrogel-based scaffolds via the McGrath method considerably improved the yield strength, ultimate strength and the compressive modulus with percentage loading as low as $7 \mathrm{wt} \%$. The improved strength may be attributed to the McGrath mineralization method (in presence of PAA) resulting in calcium carbonate crystallites growing in association with the chitosan hydrogel matrix in the form of oriented aggregation nano-sized particles. The enhanced matrix-mineral surface interaction under compression may have contributed to this enhancement. However, further addition of calcium carbonate lowered the strength and the modulus.

(c) the increasing size of the calcium carbonate crystallites within the composites may also be a contributing factor to the deterioration in the compressive modulus, yield strength and the ultimate strength of composites with the increasing mineral content. Although the McGrath mineralization (in presence of PAA) results in the formation of crystallites in association with the hydrogel matrix and via nanoparticle aggregation in all cases (when using different concentrations of precursor ions), as the concentration of the mineralization solutions is increased the resultant calcium carbonate aggregates formed are micron-sized 
structures. Along with the high mineral content, the presence of such large crystallites $(\sim 30$ $\mu \mathrm{m})$ may have resulted in the observed lower mechanical strength in the case of composites developed using $0.5 \mathrm{M}$ mineral precursor solutions in comparison to the composites fabricated using $0.1 \mathrm{M}$ precursor solutions, in which case, the mineral content was lower, and the resultant crystallites were approximately three times smaller in size.

(d) Composites with crystallites in the form of pancake-like structures (intricately associated with the polymer matrix), resulting from the aggregation of nanoparticles in association with the hydrogel matrix, have shown good mechanical strength within a range comparable to that of trabecular bone. However, this morphology of the $\mathrm{CaCO}_{3}$ crystallites did not seem to have any effect in improving the total mechanical strength of the composites in comparison to those fabricated via the McGrath method using precursor ion solutions of the same concentrations but different exposure periods, thus resulting in crystallites in the form of predominantly larger nanoparticle aggregates while still in association with the polymer matrix.

Dynamic mechanical analysis of composites under increasing loading frequency showed three distinct regions in the variation of storage and complex moduli with the loading frequency. The storage or complex moduli did not show large variations at lower frequencies $(1-9 \mathrm{~Hz})$. At increased frequencies, beyond $9 \mathrm{~Hz}$, these moduli however displayed an increasing trend, finally attaining a constant value beyond $13 \mathrm{~Hz}$. In the first two regions, the moduli values did not vary with respect to the percentage mineral content. However, as the frequency increased beyond $13 \mathrm{~Hz}$, the storage and complex moduli were higher in the case of the composites in comparison to that of the chitosan hydrogel-based scaffolds. Although the experiment was performed within the elastic limit of these samples, high-frequency loading caused some irreversible deformation in all the 3D chitosan hydrogel-based structures irrespective of the total mineral content, as identified by uniaxial compression testing performed on samples after DMA testing. Nonetheless, the testing regime did not result in complete failure of the samples and thus these depicted an initial elastic response as well under further uniaxial compression testing.

The indentation modulus and hardness as determined via nanoindentation tests in displacement control mode also showed a similar trend wherein the hardness values of the composites were higher than the pure chitosan hydrogel-based structures. No significant increase in the hardness was observed with the increasing mineral content within the final composite, however, a decreasing trend was observed in the indentation modulus. The 
indentation modulus and nanoscale hardness obtained for chitosan calcium carbonate composites fabricated by mineralization of chitosan hydrogel-based scaffolds via the McGrath method is of the same order of magnitude as that of trabecular bone.

Based on all the above observations, it is concluded that 3D printed chitosan hydrogel-based scaffolds with ordered cubic lattice-based porous architecture show good mechanical resistance to ultimate fracture under physiologically relevant stress loading conditions. Thus, making such structured chitosan- $\mathrm{CaCO}_{3}$ composites good candidates as bone graft material. Also, the results suggest that the McGrath mineralization method can be used to further accentuate the mechanical properties of 3D printed chitosan hydrogel-based scaffolds via mineral growth in association with the hydrogel matrix.

The behaviour of the scaffold in vitro in terms of biocompatibility, osteoblast adhesion and proliferation are discussed in Chapter 6. 


\subsection{Bibliography}

1. Jana, S., S.J. Florczyk, M. Leung, and M. Zhang, High-strength pristine porous chitosan scaffolds for tissue engineering. Journal of Materials Chemistry, 2012. 22(13): p. 6291-6299.

2. Khang, G., Handbook of intelligent scaffolds for tissue engineering and regenerative medicine. 2017: CRC Press.

3. Hoque, M.E., T. Nuge, T.K. Yeow, N. Nordin, and R. Prasad, Gelatin based scaffolds for tissue engineering-A review. Polymers Research Journal, 2015. 9(1): p. 15.

4. Declet, A., E. Reyes, and O. Suárez, Calcium carbonate precipitation: a review of the carbonate crystallization process and applications in bioinspired compositeS. Reviews on Advanced Materials Science, 2016. 44(1).

5. He, F., W. Ren, X. Tian, W. Liu, S. Wu, and X. Chen, Comparative study on in vivo response of porous calcium carbonate composite ceramic and biphasic calcium phosphate ceramic. Materials Science and Engineering: C, 2016. 64: p. 117-123.

6. Altay, E., T. Shahwan, and M. Tanoğlu, Morphosynthesis of $\mathrm{CaCO}_{3}$ at different reaction temperatures and the effects of PDDA, CTAB, and EDTA on the particle morphology and polymorph stability. Powder Technology, 2007. 178(3): p. 194-202.

7. Wang, S.-F., L. Shen, W.-D. Zhang, and Y.-J. Tong, Preparation and mechanical properties of chitosan/carbon nanotubes composites. Biomacromolecules, 2005. 6(6): p. 3067-3072.

8. Thein-Han, W. and R. Misra, Biomimetic chitosan-nanohydroxyapatite composite scaffolds for bone tissue engineering. Acta biomaterialia, 2009. 5(4): p. 1182-1197.

9. Venkatesan, J. and S.-K. Kim, Chitosan composites for bone tissue engineering - an overview. Marine Drugs, 2010. 8(8): p. 2252-2266.

10. Sowmya, S., P.S. Kumar, K. Chennazhi, S. Nair, H. Tamura, and J. Rangasamy, Biocompatible $\beta$-chitin hydrogel/nanobioactive glass ceramic nanocomposite scaffolds for periodontal bone regeneration. Artif. Organs, 2011. 25(1): p. 1-11.

11. Fan, H., L. Wang, K. Zhao, N. Li, Z. Shi, Z. Ge, and Z. Jin, Fabrication, mechanical properties, and biocompatibility of graphene-reinforced chitosan composites. Biomacromolecules, 2010. 11(9): p. 2345-2351.

12. Deepthi, S., J. Venkatesan, S.-K. Kim, J.D. Bumgardner, and R. Jayakumar, An overview of chitin or chitosan/nano ceramic composite scaffolds for bone tissue 
engineering. International Journal of Biological Macromolecules, 2016. 93: p. 13381353.

13. Chang, C., N. Peng, M. He, Y. Teramoto, Y. Nishio, and L. Zhang, Fabrication and properties of chitin/hydroxyapatite hybrid hydrogels as scaffold nano-materials. Carbohydrate Polymers, 2013. 91(1): p. 7-13.

14. Chesnutt, B.M., A.M. Viano, Y. Yuan, Y. Yang, T. Guda, M.R. Appleford, J.L. Ong, W.O. Haggard, and J.D. Bumgardner, Design and characterization of a novel chitosan/nanocrystalline calcium phosphate composite scaffold for bone regeneration. Journal of Biomedical Materials Research Part A, 2009. 88(2): p. 491-502.

15. Murphy, W., J. Black, and G.W. Hastings, Handbook of biomaterial properties. 2016: Springer.

16. Currey, J.D., Bones: structure and mechanics. 2002: Princeton University Press.

17. Beniash, E., Biominerals—hierarchical nanocomposites: the example of bone. Wiley Interdisciplinary Reviews: Nanomedicine and Nanobiotechnology, 2011. 3(1): p. 4769.

18. Nudelman, F., K. Pieterse, A. George, P.H. Bomans, H. Friedrich, L.J. Brylka, P.A. Hilbers, G. de With, and N.A. Sommerdijk, The role of collagen in bone apatite formation in the presence of hydroxyapatite nucleation inhibitors. Nature Materials, 2010. 9(12): p. 1004-1009.

19. Mann, S., Biomineralization: principles and concepts in bioinorganic materials chemistry. Vol. 5. 2001: Oxford University Press on Demand.

20. Ma, Y., S. Berland, J.-P. Andrieu, Q. Feng, and L. Bédouet, What is the difference in organic matrix of aragonite vs. vaterite polymorph in natural shell and pearl? Study of the pearl-forming freshwater bivalve mollusc Hyriopsis cumingii. Materials Science and Engineering: C, 2013. 33(3): p. 1521-1529.

21. Weiner, S. and H.D. Wagner, The material bone: structure-mechanical function relations. Annual Review of Materials Science, 1998. 28(1): p. 271-298.

22. Bankoff, A.D.P., Biomechanical characteristics of the bone. 2012: INTECH Open Access Publisher.

23. Yamashita, J., X. Li, B.R. Furman, H.R. Rawls, X. Wang, and C. Agrawal, Collagen and bone viscoelasticity: a dynamic mechanical analysis. Journal of Biomedical Materials Research, 2002. 63(1): p. 31-36. 
24. Linde, F., I. Hvid, and B. Pongsoipetch, Energy absorptive properties of human trabecular bone specimens during axial compression. Journal of Orthopaedic Research, 1989. 7(3): p. 432-439.

25. Fung, Y.-c., Biomechanics: mechanical properties of living tissues. 2013: Springer Science \& Business Media.

26. Dong, X.N., Y.N. Yeni, C.M. Les, and D.P. Fyhrie, Effects of end boundary conditions and specimen geometry on the viscoelastic properties of cancellous bone measured by dynamic mechanical analysis. Journal of Biomedical Materials Research Part A, 2004. 68(3): p. 573-583.

27. Yamashita, J., B.R. Furman, H.R. Rawls, X. Wang, and C. Agrawal, The use of dynamic mechanical analysis to assess the viscoelastic properties of human cortical bone. Journal of Biomedical Materials Research, 2001. 58(1): p. 47-53.

28. Bowman, S., L. Gibson, W. Hayes, and T. McMahon, Results from demineralized bone creep tests suggest that collagen is responsible for the creep behavior of bone. Journal of Biomechanical Engineering, 1999. 121(2): p. 253-258.

29. Barkey, M.C.b.M. AEM 251-Preload Correction of Stress-Strain Curves. 2016; Available from: https://www.youtube.com/watch?v=qrJByE1P5cE.

30. Gebauer, D., H. Cölfen, A. Verch, and M. Antonietti, The multiple roles of additives in CaCO3 crystallization: A quantitative case study. Advanced Materials, 2009. 21(4): p. 435-439.

31. Vaz, M.F., H. Canhão, and J.E. Fonseca, Bone: a composite natural material, in Advances in Composite Materials-Analysis of Natural and Man-Made Materials. 2011, InTech.

32. Fischer-Cripps, A.C., Factors Affecting Nanoindentation Test Data. 2000: Springer.

33. Qian, L., M. Li, Z. Zhou, H. Yang, and X. Shi, Comparison of nano-indentation hardness to microhardness. Surface and Coatings Technology, 2005. 195(2): p. 264271.

34. Sattari, M., M. Naimi-Jamal, and A. Khavandi, Interphase evaluation and nanomechanical responses of UHMWPE/SCF/nano-SiO 2 hybrid composites. Polymer Testing, 2014. 38: p. 26-34.

35. Keaveny, T.M. and W.C. Hayes, Mechanical properties of cortical and trabecular bone. Bone, 1993. 7: p. 285-344.

36. Reilly, D.T. and A.H. Burstein, The elastic and ultimate properties of compact bone tissue. Journal of Bomechanics, 1975. 8(6): p. 393IN9397-396IN11405. 
37. Gibson, L.J. and M.F. Ashby, Cellular solids: structure and properties. 1999: Cambridge University Press.

38. An, Y.H. and R.A. Draughn, Mechanical testing of bone and the bone-implant interface. 1999: CRC Press.

39. Zhang, Y. and M. Zhang, Synthesis and characterization of macroporous chitosan/calcium phosphate composite scaffolds for tissue engineering. Journal of Biomedical Materials Research Part A, 2001. 55(3): p. 304-312.

40. Kalita, S.J., S. Bose, H.L. Hosick, and A. Bandyopadhyay, Development of controlled porosity polymer-ceramic composite scaffolds via fused deposition modeling. Materials Science and Engineering: C, 2003. 23(5): p. 611-620.

41. Zhang, Y. and M. Zhang, Three-dimensional macroporous calcium phosphate bioceramics with nested chitosan sponges for load-bearing bone implants. Journal of Biomedical Materials Research Part A, 2002. 61(1): p. 1-8.

42. Karageorgiou, V. and D. Kaplan, Porosity of $3 D$ biomaterial scaffolds and osteogenesis. Biomaterials, 2005. 26(27): p. 5474-5491.

43. Hing, K.A., Bioceramic bone graft substitutes: influence of porosity and chemistry. International Journal of Applied Ceramic Technology, 2005. 2(3): p. 184-199.

44. Athanasiou, K., C.-F. Zhu, D. Lanctot, C. Agrawal, and X. Wang, Fundamentals of biomechanics in tissue engineering of bone. Tissue Engineering, 2000. 6(4): p. 361381.

45. Sun, J. and B. Bhushan, Hierarchical structure and mechanical properties of nacre: a review. RSC Advances, 2012. 2(20): p. 7617-7632.

46. Jimenez-Palomar, I., A. Shipov, R. Shahar, and A.H. Barber, Mechanical behavior of osteoporotic bone at sub-lamellar length scales. Frontiers in Materials, 2015. 2: p. 9.

47. Abé, H., K. Hayashi, and M. Sato, Data book on mechanical properties of living cells, tissues, and organs. 1996: Springer.

48. Lam, C.-K., H.-y. Cheung, K.-t. Lau, L.-m. Zhou, M.-w. Ho, and D. Hui, Cluster size effect in hardness of nanoclay/epoxy composites. Composites Part B: Engineering, 2005. 36(3): p. 263-269.

49. Yasmin, A. and I.M. Daniel, Mechanical and thermal properties of graphite platelet/epoxy composites. Polymer, 2004. 45(24): p. 8211-8219.

50. Sayer, M., Elastic properties and buckling load evaluation of ceramic particles filled glass/epoxy composites. Composites Part B: Engineering, 2014. 59: p. 12-20. 
51. Asi, O., Mechanical Properties of Glass-Fiber Reinforced Epoxy Composites Filled with Al $2 O 3$ Particles. Journal of Reinforced Plastics And Composites, 2009. 28(23): p. 2861-2867.

52. Yang, H.-S., H.-J. Kim, J. Son, H.-J. Park, B.-J. Lee, and T.-S. Hwang, Rice-huskflour filled polypropylene composites; mechanical and morphological study. Composite Structures, 2004. 63(3): p. 305-312.

53. Levengood, S.K.L. and M. Zhang, Chitosan-based scaffolds for bone tissue engineering. Journal of Materials Chemistry B, 2014. 2(21): p. 3161-3184.

54. Swain, S.K., S. Dash, S.K. Kisku, and R.K. Singh, Thermal and oxygen barrier properties of chitosan bionanocomposites by reinforcement of calcium carbonate nanopowder. Journal of Materials Science \& Technology, 2014. 30(8): p. 791-795.

55. Nguyen, T.T.B., S. Hein, C.H. Ng, and W.F. Stevens, Molecular stability of chitosan in acid solutions stored at various conditions. Journal of Applied Polymer Science, 2008. 107(4): p. 2588-2593.

56. Hvid, I., P. Christensen, J. Søndergaard, P.B. Christensen, and C.G. Larsen, Compressive Strength of Tibial Cancellous Bone: Instron ${ }^{\circledR}$ and Osteopenetrometer Measurements in an Autopsy Material. Acta Orthopaedica Scandinavica, 1983. 54(6): p. 819-825.

57. Kopperdahl, D.L. and T.M. Keaveny, Yield strain behavior of trabecular bone. Journal of Biomechanics, 1998. 31(7): p. 601-608.

58. Goulet, R.W., S.A. Goldstein, M.J. Ciarelli, J.L. Kuhn, M. Brown, and L. Feldkamp, The relationship between the structural and orthogonal compressive properties of trabecular bone. Journal of Biomechanics, 1994. 27(4): p. 375379-377389.

59. Fu, S.-Y., X.-Q. Feng, B. Lauke, and Y.-W. Mai, Effects of particle size, particle/matrix interface adhesion and particle loading on mechanical properties of particulatepolymer composites. Composites Part B: Engineering, 2008. 39(6): p. 933-961.

60. Munro, N.H. and K.M. McGrath, How important is polyelectrolyte complex formation in biomimetic mineralisation? Manipulation via alcohol addition. Dalton Transactions, 2013. 42(23): p. 8259-8269.

61. Lynch, M.E. and C. Fischbach, Biomechanical forces in the skeleton and their relevance to bone metastasis: Biology and engineering considerations. Advanced Drug Delivery Reviews, 2014. 79: p. 119-134.

62. Sadeghi, H., D.M. Espino, and D.E. Shepherd, Variation in viscoelastic properties of bovine articular cartilage below, up to and above healthy gait-relevant loading 
frequencies. Proceedings of the Institution of Mechanical Engineers, Part H: Journal of Engineering in Medicine, 2015. 229(2): p. 115-123.

63. Lee, S., M. Porter, S. Wasko, G. Lau, P.-Y. Chen, E.E. Novitskaya, A.P. Tomsia, A. Almutairi, M.A. Meyers, and J. McKittrick, Potential bone replacement materials prepared by two methods. MRS Online Proceedings Library Archive, 2012. 1418.

64. Fulcher, G.R., D.W. Hukins, and D.E. Shepherd, Viscoelastic properties of bovine articular cartilage attached to subchondral bone at high frequencies. BMC musculoskeletal disorders, 2009. 10(1): p. 61.

65. Lewis, G. and J.S. Nyman, The use of nanoindentation for characterizing the properties of mineralized hard tissues: State-of-the art review. Journal of Biomedical Materials Research Part B: Applied Biomaterials, 2008. 87(1): p. 286-301.

66. Rho, J.Y., M.E. Roy, T.Y. Tsui, and G.M. Pharr, Elastic properties of microstructural components of human bone tissue as measured by nanoindentation. Journal of Biomedical Materials Research Part A, 1999. 45(1): p. 48-54.

67. Ho, S.P., H. Goodis, M. Balooch, G. Nonomura, S.J. Marshall, and G. Marshall, The effect of sample preparation technique on determination of structure and nanomechanical properties of human cementum hard tissue. Biomaterials, 2004. 25(19): p. 4847-4857. 


\section{Biological Performance}

\subsection{Introduction}

Bone tissue is known for its ability to repair and regenerate, however when the defect size is too large, often the intervention of a scaffold is required, which can help bridge the defect, provide an environment that facilities activation of osteoblast cells and mechanical support during the regeneration process. The cells that constitute bone include osteoblasts, osteoclasts, and osteocytes [1]. Osteoblasts are responsible for producing and mineralizing new bone matrix, osteocytes maintain the matrix, and osteoclasts are responsible for resorption of the bone matrix. Thus, an ideal bone tissue engineering scaffold can be developed by combining osteoconductive scaffolds, with osteoinductive growth factors and osteoblasts to repair and regenerate bone [2]. Such scaffolds are also expected to facilitate vascularization upon in vivo implantation at the defect site.

The 3D scaffold is an important component in bone tissue engineering that serves as a template for osteoblasts to adhere and initiate new bone formation while providing structural support during the regeneration process. Thus, apart from mechanical compatibility, an ideal bone tissue engineering scaffold should (1) have appropriate porosity with macro and micro pores facilitating solvent diffusion and ion exchange; (2) interconnected porosity for tissue in-growth and vascularization; (3) controlled degradation kinetics with respect to bone regrowth without the formation of any toxic residues or inflammatory response; (4) have functionalities that facilitate adhesion and proliferation of osteoblasts for formation and mineralization of the bone matrix; and (5) provide a sterile environment for in vitro cell seeding (in the case of pre-seeded scaffolds that are then implanted at the defect site in vivo) [3-5]. Scaffolds can also be chemically modified to act as carriers of cytokines such as bone morphogenetic proteins (BMPs), insulin-like growth factors (IGFs) and transforming growth factors (TGFs) that facilitate osteoinduction [6].

It is well established that cells show enhanced performance in a 3D microenvironment that resembles the in vivo conditions, compared to 2D templates [7]. As such, most of the recent studies in the field of bone tissue engineering have been directed towards developing 3D 
scaffolds that are biologically active. Other than chitosan, a natural biocompatible polymer used in this research, 3D porous biocompatible scaffolds fabricated using various materials have been reported in literature for bone tissue engineering applications ranging from (a) metal-based porous scaffolds of titanium, selective layer sintered matrices of titanium alloys and steel [8,9]; (b) ceramics and polymer-ceramic composites of calcium phosphate (hydroxyapatite, tricalcium phosphate and biphasic calcium phosphate), bioglass and polymers such as (PLGA) [10-12]; (c) biopolymer composites of alginate, collagen, gelatin $[13,14]$; to (d) synthetic polymer-based 3D porous scaffolds developed using polycaprolactone, polylactic acid (PLA), polyglycolic acid (PGA), copolymers of PLA and PGA (PLGA) $[15,16]$. Among these, the composites of natural polymers (such as collagen, gelatin, chitosan, silk fibroin etc) and ceramics such as calcium phosphates have gained considerable popularity owing to the fact that biopolymers (carbohydrate or protein-based) have functional groups similar to the extracellular matrix, are chemically versatile, and have known biocompatibility without invoking any immunological reactions in vivo; and that calcium phosphates are bioresorbable, osteoconductive, and biocompatible [17, 18]. Furthermore, these properties are found to be more effective when at least one of these phases (organic or inorganic) are in the form of nanoparticles, or nanofibers thus, effectively generating a biocompatible nanocomposite. In nanocomposites, the interaction between the two phases is strong thus imparting enhanced mechanical stability, while maintaining the favourable osteoconductivity and biocompatibility of its constituents, in turn influencing protein adsorption, cells adhesion, proliferation and differentiation for bone regeneration [19].

Chitosan is a very versatile polymer. Literature shows multiple examples of 3D chitosan hydrogels and composites (mechanical blends or biomimetic composites) exhibiting excellent adhesion, proliferation and differentiation of osteoblast cells, facilitating neovascularization, cell infiltration and induced bone formation in vitro and in vivo [20$23]$. Thus, the probability of the 3D printed porous chitosan hydrogel-based scaffold and composites developed in this research being bioactive is extremely high. Furthermore, the presence of nanoparticles of calcium carbonate intimately interacting with the polymer matrix enhances this prospect.

It has been shown in Chapter 5 that the 3D printed chitosan hydrogel-based composites developed in this research exhibit mechanical properties (compressive modulus, and resistance to complete fracture under compression) in the order of magnitude that is 
comparable to that of trabecular bone and that it offers considerable resistance to yielding and complete fracture when subjected to physiologically relevant stress and strain. Now, it is important to evaluate the biological performance of these materials in in vitro conditions in order to get a better understanding of the applicability of this material in applications as load-bearing bone grafts.

In this Chapter, the in vitro interaction of the 3D printed chitosan hydrogel-based scaffolds and composites fabricated by mineralization of chitosan hydrogel-based scaffolds via the McGrath method with primary osteoblast cells is described. In particular, osteoblast cell adhesion on the 3D scaffold surface, viability and proliferation in the presence of $3 \mathrm{D}$ composites as a function of increasing mineral content in the composites are investigated via Alamar Blue cell proliferation assay and confocal microscopy (specific stains used to identity cell adhesion). 


\subsection{Materials and methods}

The materials and methods used to fabricate 3D chitosan hydrogel-based scaffold are the same as described in Chapter 3. For biological tests, scaffolds $30 \mathrm{~mm}$ diameter $\times 3 \mathrm{~mm}$ high with $1.5 \mathrm{~mm} \times 1.5 \mathrm{~mm}$ macropores (in wet state) based on the CAD model described in Chapter 2, were fabricated using $5 \% \mathrm{w} / \mathrm{v}$ chitosan hydrogel (in the presence of $2 \% \mathrm{v} / \mathrm{v}$ acetic acid) as the printing ink in our custom-designed 3D printer at a printing speed of $12 \mathrm{~mm} / \mathrm{s}$. The printing ink was prepared by dissolving $5 \% \mathrm{w} / \mathrm{v}$ chitosan in $2 \% \mathrm{v} / \mathrm{v}$ acetic acid solution under continuous stirring $(350 \mathrm{rpm})$ for $12 \mathrm{~h}$ and then homogenising the solution at 4000 rpm. Note that the samples used for these tests are substantially larger than those used throughout the rest of this thesis.

The $\mathrm{pH}$ neutralized 3D chitosan hydrogel-based scaffolds were then mineralized via the McGrath method (24 h alternate soaking +1 week Kitano soaking) in the presence of $2.5 \%$ w/w polyacrylic acid (PAA) (w.r.t dry weight of chitosan used) and then air-dried. Composites with two different total mineral contents were fabricated via this method in this study, namely, low mineral chitosan- $\mathrm{CaCO}_{3}$ composites (McGrath mineralization in the presence of $100 \mathrm{mM}$ precursor ion solution) $\left(\sim 14.9 \pm 1.6 \% \mathrm{CaCO}_{3}\right)$ and high mineral chitosan- $\mathrm{CaCO}_{3}$ (McGrath mineralization in the presence of $500 \mathrm{mM}$ precursor ion solution) $\left(\sim 36.8 \pm 2.1 \% \mathrm{CaCO}_{3}\right)$. Non-mineralized chitosan hydrogel-based scaffolds were used as the control.

Only two different variants of the mineralized samples were chosen for this study considering that the mechanical properties of composites did not vary extensively with the increase in mineral content (refer to Chapter 5). Data obtained from most of the mechanical tests showed that a statistically relevant variation in mechanical properties was observed between (1) non-mineralized chitosan hydrogel-based scaffolds and composites fabricated using $0.5 \mathrm{M}$ precursor ion solutions; and (2) composites with low mineral content fabricated using $0.1 \mathrm{M}$ precursor ion solution and those with high mineral content fabricated using 0.5 $\mathrm{M}$ precursor ion solutions. Thus, composites with intermediate mineral content i.e. those fabricated using McGrath mineralization method in the presence of $0.25 \mathrm{M}$ precursor ion solutions ( $24 \mathrm{~h}$ alternate soaking +1 week Kitano) were not tested for biological properties.

It is important to note that, for all biological tests as-fabricated chitosan hydrogel-based scaffolds and composites are used i.e. no sample preparation is involved. Thus, the total mineral content of the composites used in this case is not directly comparable to those used 
for mechanical testing experiments as those samples required filing, leading to the loss of some of the inorganic content. Nonetheless, based on the data obtained from both the tests, an optimal range of the total mineral content of the composites suitable for enhanced biological performance together with maintaining appropriate mechanical properties will be able to be obtained.

\subsubsection{Materials for biological testing}

The Alpha-MEM (Minimum Essential Medium Eagle) culture media and foetal bovine serum (FBS) were purchased from Gibco ${ }^{\circledR}$, Life Technologies; phosphate buffered saline (PBS), 4,6-diamidino-2-phenylindole (fluorescent dye) (DAPI), Alexa Fluor 594 Phalloidin (red stain), and Triton X-100 were purchased from Sigma-Aldric; Alamar Blue stain was purchased from Molecular Probes, Life Technologies; and MC3T3 primary osteoblast cells were procured from American Tissue Type Collection and were used as obtained from the manufacturers.

\subsubsection{In vitro cell viability test}

To study the biological performance of the 3D printed chitosan hydrogel-based scaffolds and composites in a bone-like microenvironment as a function of their total mineral content and period of incubation, the samples (air-dried) were incubated in culture media with MC3T3 primary osteoblast cells for a period of 14 days and assessed for cell proliferation. The cell viability/ proliferation rate was quantized via Alamar Blue assay performed at different time points (day 1, 3, 7 and 14) during the incubation period. Four samples of each type were tested against two samples of each type as negative controls (scaffolds in media with no cells present). The experiment was carried out three times to account for biological variability.

Air-dried 3D printed chitosan hydrogel-based scaffolds/composites were initially UV sterilized on all surfaces for $30 \mathrm{~min}$ each and then transferred to a 24 -well culture plate. The scaffolds were then soaked in PBS (1 mL) for $30 \mathrm{~min}$ at room temperature and incubated in $1 \mathrm{~mL}$ of alpha-MEM media with $5 \% \mathrm{v} / \mathrm{v}$ FBS for $30 \mathrm{~min}$ in the cell culture incubator (37 ${ }^{\circ} \mathrm{C}$ incubator with $5 \% \mathrm{CO}_{2}$ ). It was observed that all samples swell when incubated in the culture media. The degree of swelling observed in individual scaffolds varied with mineral content. Thus, in order to maintain uniform dimensions for each of the samples tested with the cells, standardized samples were obtained from the swollen scaffolds by using a 
sterilized hole-punch with a $10 \mathrm{~mm}$ diameter hole. The scaffolds were then incubated in fresh culture media containing 5\% v/v FBS and 50,000 cells/scaffold.

The cell proliferation/viability is quantitatively analysed via the Alamar Blue assay and spectrophotometry. The Alamar Blue reagent is an oxidized form of a redox indicator (resazurin) which is normally blue in colour and non-fluorescent. When incubated with live cells, the reagent changes colour from blue to red and becomes fluorescent in response to the cellular metabolic activities. The intensity of fluorescence thus produced is proportional to the number of living cells present in the system. For the Alamar blue assay, $1 \mathrm{~mL}$ of $10 \%$ v/v Alamar Blue solution was prepared in culture media (5\% v/v FBS + alpha-MEM) and added to appropriate wells in a 24-well culture plate (new plate). To quantitatively analyse the cell proliferation rate after $24 \mathrm{~h}$ incubation with the 3D scaffolds (day 1 ), the scaffolds then were transferred to appropriate wells of the new plate with Alamar Blue solution with sterile forceps and incubated for $4 \mathrm{~h}$. In this study, cells cultured in the absence of scaffolds were used as a positive control.

After $4 \mathrm{~h}$ incubation in the Alamar Blue media, $200 \mu \mathrm{L}$ of the media from each well was transferred to appropriate wells in a 96-well culture plate and the fluorescence intensity was measured at $540 \mathrm{~nm}$ excitation wavelength and $590 \mathrm{~nm}$ emission wavelength using a Synergy 2 multi-mode microplate reader (BioTek Instruments Inc., USA). The cell viability is expressed as a percentage of the ratio of fluorescence intensity obtained for cells incubated with the test samples to that of the negative control (test samples incubated without cells).

The remaining Alamar Blue solution was removed, the test samples were washed twice with $1 \mathrm{~mL}$ of fresh media (5\% v/v FBS + alpha-MEM) and then incubated in $1 \mathrm{~mL}$ of fresh media until the next time point (day 3). The same step was followed for cell viability analysis on day 3 , day 7 and day 14 .

\subsection{3: Histology and imaging}

The data obtained from the Alamar Blue assay is co-related to images taken at specific time points using confocal microscopy. For imaging, two samples of each type were incubated with primary osteoblast cells in culture media (alpha-MEM $+5 \% \mathrm{v} / \mathrm{v}$ FBS) for 1, 3, 7 and 14 days. Different samples of each type were used for imaging and the proliferation analysis described above. It is assumed that the two protocols followed do not significantly affect the results. 
For confocal imaging, the 3D chitosan hydrogel-based scaffolds and composites incubated with cells for various time periods were washed twice with $1 \mathrm{~mL}$ PBS and then fixed in 1 $\mathrm{mL}$ of $10 \% \mathrm{v} / \mathrm{v}$ formalin (in PBS) for $20 \mathrm{~min}$ at room temperature in a 24 -well plate. The washing process ensured that the cells that are not attached to the scaffolds are removed and that the remaining ones are in close association with the scaffold. The fixed samples were then washed twice with $1 \mathrm{~mL}$ each of PBS and treated with $1 \mathrm{~mL}$ of $0.5 \% \mathrm{v} / \mathrm{v}$ triton $\mathrm{X} 100$ (in PBS) overnight (at $4{ }^{\circ} \mathrm{C}$ ). This step permeabilises the cell membrane of the osteoblast cells attached to the scaffold. The samples were then stained with Alexa Fluor 594 Phalloidin (red stain) in order to stain the F-actin filaments present in the cells. A 1:40 dilution of the stain was prepared in PBS and $500 \mu \mathrm{L}$ of the solution was added to the wells containing the samples (overnight soaking at $4{ }^{\circ} \mathrm{C}$ ). In order to stain the cell nucleus, these samples were washed three times with $1 \mathrm{~mL}$ of PBS to remove the excess red stain and treated with DAPI (blue stain). Samples were treated with $300 \mathrm{nM}$ DAPI (in PBS) for $3 \mathrm{~h}$ (at $4{ }^{\circ} \mathrm{C}$ ). After staining, the samples were washed twice with $1 \mathrm{~mL}$ of PBS and stored in 1 $\mathrm{mL}$ of fresh PBS at $4{ }^{\circ} \mathrm{C}$ until further use. Samples were imaged using a Carl Zeiss LSM 710 confocal microscope at $10 \times$ and $20 \times$ magnification.

\subsection{4: Statistical analysis}

All experiments were performed three times, and the values reported are the means \pm the standard error. Two-way ANOVA was used to assess the statistical significance of the results for all the measurements and a Tukey post hoc analysis was performed to identify the point of variation $(p<0.05)$. The analysis was performed using PRISM 6 graph pad software. 


\subsection{Results and discussions}

The recommended sample dimensions for biological testing when using 3D samples and a 24-well culture plate, is roughly, $15.6 \mathrm{~mm}$ diameter discs, with a height of 1-2 mm. These dimensions ensure that the test samples fit in the wells of a 24-well culture plate and that the samples are fully submerged in the culture media. Accordingly, based on the previous observation that chitosan hydrogel-based scaffolds and the composites undergo considerable shrinkage during the air-drying process (refer to Chapter 2), 3D printed chitosan hydrogel-based scaffolds $30 \mathrm{~mm}$ in diameter $\times 3 \mathrm{~mm}$ high, were fabricated with $1.5 \mathrm{~mm} \times 1.5 \mathrm{~mm}$ macropores (wet state) such that after air-drying the final dimensions of the test samples conformed to the prerequisite values. The dimensions of the dried scaffolds (pure hydrogel-based and composites) could not be accurately measured due to the curling effect observed as shown in Figure 6.1, however, prior to biological testing, it was ensured that all test samples fitted well into the 24 -well culture plates. It was observed that air dying of mineralized samples resulted in slightly larger samples compared to non-mineralized scaffolds. This may be due to the presence of calcium carbonate crystallites acting as filler particles that restrict the extensive coiling of the polymer chains during the air-drying process.

\subsubsection{Scaffold swelling}

During the cell viability/proliferation testing process, it was observed that initial incubation of the test samples in PBS and culture media ( $\mathrm{pH} 7.2$ in each case) results in swelling of the samples. The degree of swelling (based on variation in the dimensions) varied with the mineral content of the samples. The degree of swelling observed was in the order high mineral > low mineral > no mineral (pure chitosan hydrogel-based samples) as shown in Figure 6.1. The observed swelling could be attributed to the micro and nanopores that may be regained during the soaking process as described in Chapter 2.

The variation in the swelling across the samples may be attributed to the variation in the degree of shrinkage occurring during the air-drying process in samples with varying calcium carbonate content and the subsequent proportional variation in the diffusion dependent rehydration process. These particles act as fillers that restrict the coiling and aggregation of polymer chains during air-drying, thus reducing the overall shrinkage in the structure, in comparison to the un-restricted polymer coiling and aggregation occurring in samples without any mineral phase. The increased porosity and the resultant higher surface area 
facilitates enhanced solvent diffusion of the culture media throughout the test samples. This may have contributed to the higher swelling observed in the mineralized samples, more prominently so, in those with very high percentage calcium carbonate content. In the case of the composites with high mineral content, the dimensions in the swollen state are comparable to that of as-fabricated wet chitosan hydrogel-based scaffolds (Figure 6.1). Thus, in this case, it can be assumed that the swelling may have resulted in regaining most of the "lost" porosity as a result of the drying process. The extensive swelling observed may aid in enhanced cell proliferation and growth.

\section{Air dried Samples}
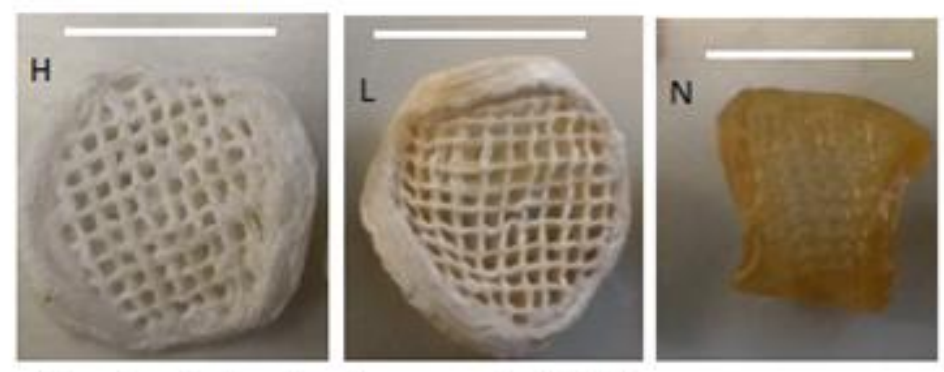

\section{As-fabricated 3D chitosan hydrogel-based scaffold}

After incubation in culture media $(0.5 \mathrm{~h})$
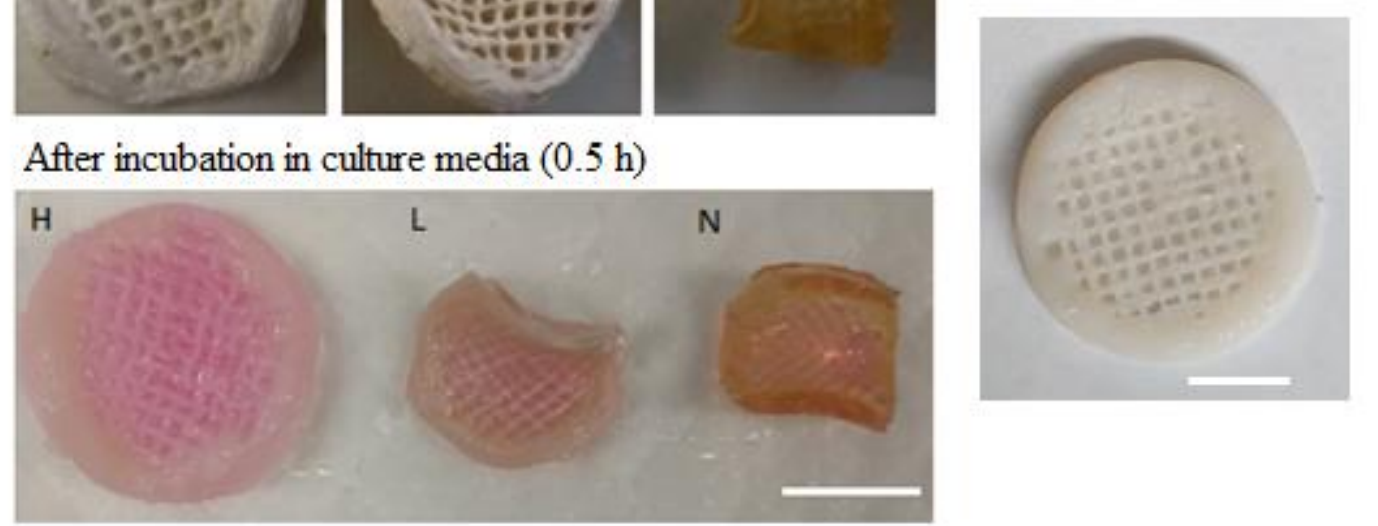

Figure 6.1: Representative optical images of the swelling observed in air-dried 3D chitosan hydrogel-based scaffolds and composites after incubation in the culture media (pH 7.2) containing 5\% v/v FBS. Samples labelled ' $H$ ' represents composites with high mineral developed by mineralization of chitosan hydrogel-based scaffolds using $0.5 \mathrm{M}$ precursor ion solutions via the via McGrath; samples labelled ' $L$ ' represents composites by mineralization of chitosan hydrogel-based scaffolds using 0.1 M precursor ion solutions via the McGrath; and samples labelled ' $N$ ' represents chitosan hydrogel-based samples (no mineral). Also shown for comparison is the optical image of the as-fabricated chitosan hydrogel-based scaffold in wet condition. The scale bar is $1 \mathrm{~cm}$.

In order to maintain uniform dimensions for all the samples tested with the cells, a sterilized $10 \mathrm{~mm}$ diameter hole-punch was used to obtain uniformly-sized samples from the swollen samples, which were then incubated with osteoblast cells. It was observed that after the 
initial swelling (during the first incubation in culture media without cells), no further swelling occurred in any of the test samples and the samples remained intact throughout the testing process (over 14 days).

It should be noted that the variation in the degree of swelling occurring in air-dried chitosan hydrogel-based scaffolds and composites with respect to the mineral content, causes the dimensions of the printed macropores and thus the porosity of the final tested samples (after punching) to vary slightly among each other. Thus, these parameters together with the total mineral content of the tested samples, as explained earlier, are not comparable to those of the samples used for the mechanical testing.

Furthermore, it is important to note that the variation in the porosity across the test samples may also be a contributing factor to any differences observed in the biological performance of these 3D structures with the osteoblast cells. These parameters are however not considered as prominent variables in this analysis considering that the pore size, porosity and pore interconnectivity is still within a range applicable for osteoblast cell proliferation (refer to Section 5.3.1 (a) of Chapter 5).

\subsubsection{Cell viability, proliferation and adhesion}

Data from the Alamar Blue assay revealed that chitosan hydrogel-based scaffolds and composites are biologically active and that they support viability and proliferation of osteoblast cells. Figure 6.2 shows the variation in the cell viability (\%) at different time points as a function of the total mineral content in the scaffolds.

Data indicates that the cell proliferation rate increased with time in the presence of composites with low mineral content, i.e. those fabricated by mineralization (in the presence of $2.5 \%$ w/w PAA) of chitosan hydrogel-based scaffolds via the McGrath method in the presence of $0.1 \mathrm{M}$ precursor ions solution ( $24 \mathrm{~h}$ alternate soaking +1 week Kitano soaking). A statistically significant increase in the number of osteoblast cells was observed only after 14 days of incubation with the test samples. The proliferation rate was highest when incubated with low mineral content samples in comparison to the non-mineralization samples (no mineral versus low mineral; adjusted $\mathrm{p}$ value $=0.0020$ ) and composites with high mineral content (low mineral versus high mineral adjusted $\mathrm{p}$ value $=0.0126$ ). No significant difference was observed in the cell proliferation rate when osteoblasts were incubated with non-mineralized chitosan hydrogel-based scaffolds and composites with high mineral content. 


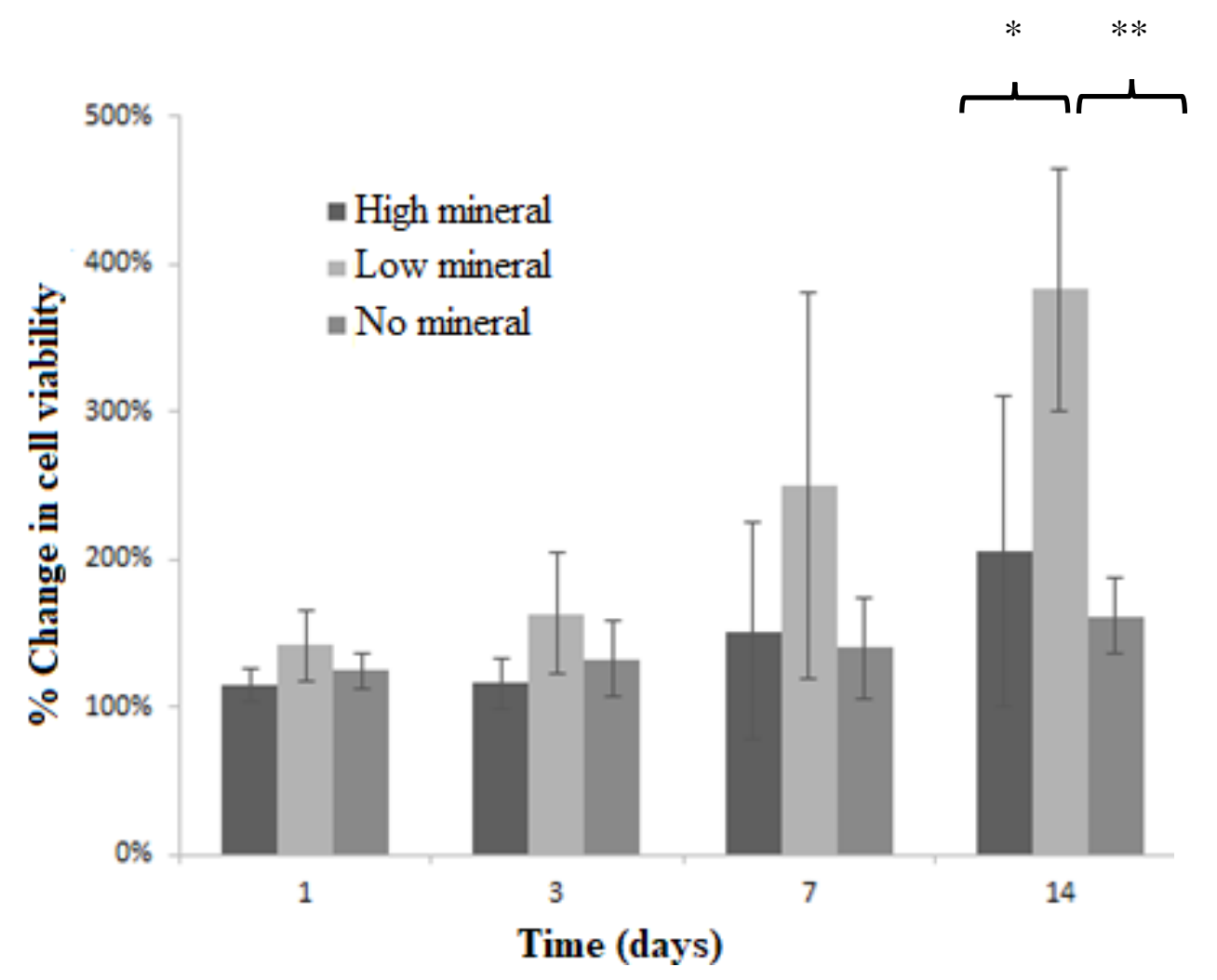

Figure 6.2: Changes in the osteoblast cell viability/proliferation (percentage over a 14-day culture period in response to the mineral content of $3 D$ printed chitosan hydrogel-based scaffolds. Composites with high mineral content were developed via McGrath mineralization using $0.5 \mathrm{M}$ precursor ion solutions and composites with low mineral content were developed via the McGrath mineralization using 0.1 M precursor ion. Four replicates of each variant were tested under similar conditions. The error bars represent the standard deviation of the values obtained from the mean of those obtained for four replicates of each type (repeated three times). Adjusted p value $\leq 0.05(*)$ and $\leq 0.01$ (**).

Furthermore, confocal microscope images revealed that the osteoblasts adhered to the surface of the samples as indicated by the presence of interconnected actin filaments on the scaffold surface (stained red or green in Figure 6.3). Figure 6.3 shows the confocal images of 3D chitosan hydrogel-based scaffolds with varying mineral content after 14 days of incubation with osteoblasts. Images are acquired at different settings in order to obtain a better visualization of the cells adhered to the scaffolds. Despite the Alamar Blue tests indicting that a high proliferation rate was observed when osteoblasts were incubated for 14 days with composites with low mineral content, the confocal microscope images obtained at this time point did not show any difference between the cells on high or low mineral 
scaffolds (identified by the number of nuclei, stained blue, on the scaffold surface in Figure $6.3(\mathrm{~d}, \mathrm{e}))$.

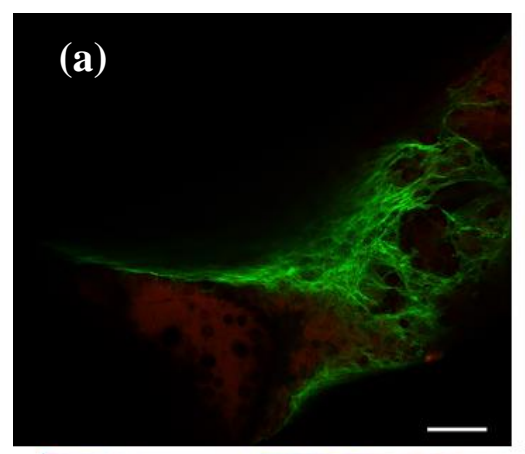

(d)

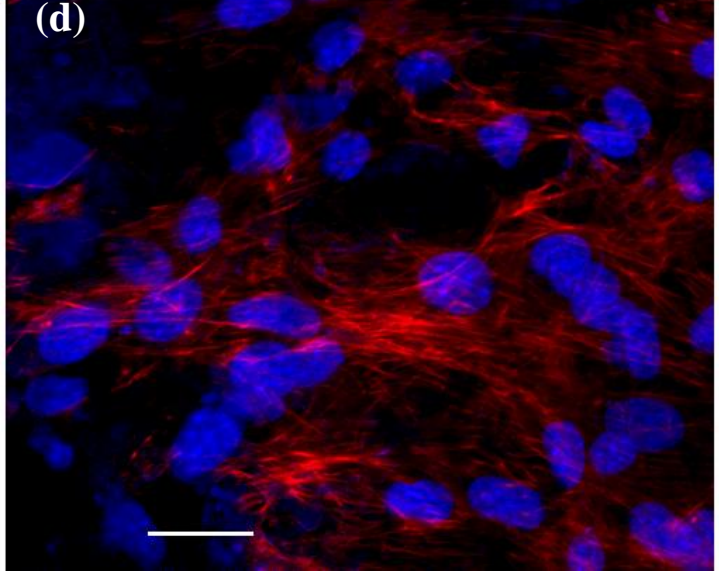

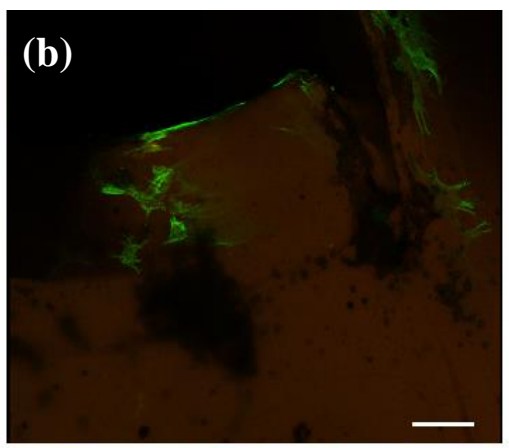

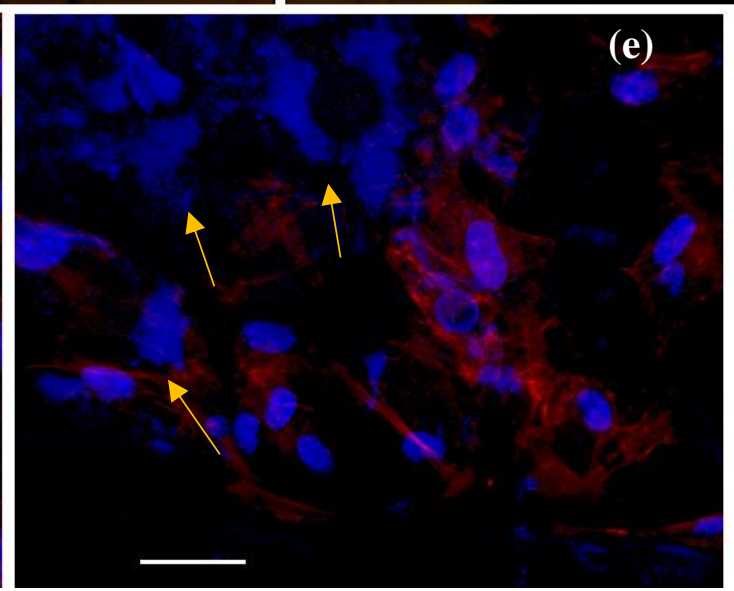

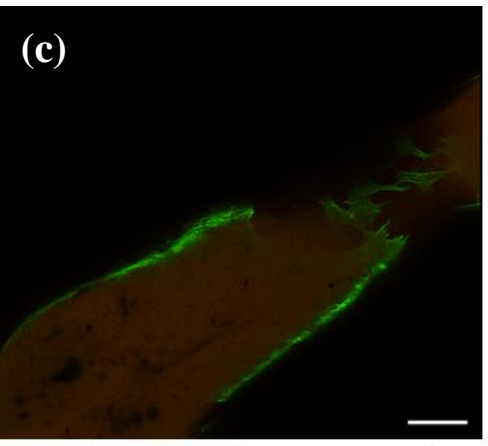

e)

Figure 6.3: Confocal microscopy images of 3D samples obtained at two different image acquisition settings. On the top are images of (a) chitosan hydrogel-based scaffolds with high mineral content; (b) composites with low mineral content; and (c) chitosan hydrogel-

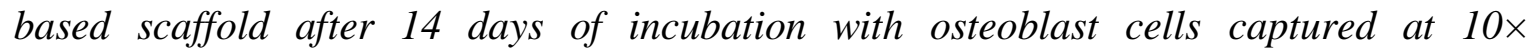
magnification. In these images, the green regions are the actin filaments and the red regions are the scaffold. Here, the DAPI staining is not visible as it was masked by the high intensity background. On the bottom are high magnification images $(20 \times)$ of $(d)$ chitosan hydrogelbased scaffolds with high mineral content; and (e) chitosan hydrogel-based scaffolds with low mineral. In these images, the blue regions correspond to DAPI stained cell nuclei and the red regions are actin filaments. The blue patches (shown by yellow arrows) observed in (b) correspond to the DAPI stained scaffold material. At this microscope setting, no cells were captured on the non-mineralized scaffolds and so the images acquired were blank (not shown). In (b), (c), (d), and (e) very few cells adhered to the surface and so the images shown are the best possible representations of the osteoblast adhered scaffold surface as observed in each case. Scale bar for the images on the top is $100 \mu \mathrm{m}$ and for those on the bottom is $50 \mu \mathrm{m}$. 
The observed discrepancy may be attributed to the fact that the sample preparation steps followed in order to quantitative and qualitatively analyse the biological performance of the scaffolds were different in each case (for example, different staining protocols used will have different effects on the chitosan polymer chains). Another contributing factor could be the variation in the nanotopography of the sample surface which is directly interacting with the cells (topography changes when dried samples swell and with varying mineral content). It is noted that the fabrication protocol used in this research is not optimized in terms of generating 3D scaffolds that have a uniform nanotopography. It is well known that such topographic variations affect the extent of interaction of the cells with a scaffold material $[24,25]$. Thus, in the case of chitosan hydrogel-based scaffolds with varying mineral content, despite the environmental conditions being viable for osteoblast adhesion and proliferation, such topographic variations may have in some cases adversely affected the extent of cell-scaffold interaction and thus were washed off during sample preparation.

Similarly, although the staining protocols followed in this research are universally accepted for characterising cellular features, these processes, may not have a similar effect on all scaffold materials. It is possible that these processes may affect the chemistry of the scaffold material and in turn affect the cell-scaffold interaction. Hence, it is suggested that biological tests need to be standardised not only with respect to the cells types but also with respect to the scaffold material involved such that the protocol followed does not affect (neither enhance nor adversely affect) the cell-matrix interaction.

Nonetheless, it is evident from the confocal images and the proliferation tests that osteoblasts show enhanced viability and proliferation in the presence of $3 \mathrm{D}$ printed chitosan hydrogel-based scaffolds and that the chitosan hydrogel-based scaffolds facilitate appropriate osteoblast adhesion. 


\subsection{Conclusion}

The in vitro biological performance of 3D printed chitosan hydrogel-based scaffolds incubated with osteoblasts cells, in terms of the cell viability, proliferation rate and adhesion on the scaffolds, as a function of the total mineral content was investigated.

It was found that the composites and chitosan hydrogel-based scaffolds swell initially when incubated with the cell culture media. The degree of swelling increased with the total mineral content of the air-dried chitosan hydrogel-based scaffolds. The observed swelling may be attributed to the increased scaffold porosity and surface area available for interaction with the solvent as a result of restricted polymer coiling and aggregation occurring during the air-drying process when calcium carbonate particles are present in the form of fillers interlacing the biopolymer matrix. In the case of composites with high content, the swollen structures were dimensionally comparable to that of the as-fabricated chitosan hydrogelbased scaffolds (no mineral, in the wet state), suggesting that most of the "lost" porosity as a result of the drying process may have been regained during swelling. It was also observed that swelling did not result in the complete dissolution of the scaffold and that the structures remained intact throughout the experiment. However, for future application of these scaffolds as in vivo bone grafts, it is important to gain a better understanding of the swelling and degradation kinetics of these scaffolds as (1) the implanted scaffold must be geometrically compatible to the implant site; and (2) swelling affects the mechanical properties of the scaffold.

Cell viability tests revealed that chitosan hydrogel-based scaffolds and composites are biocompatible, non-toxic and induce growth and proliferation of osteoblasts within 14 days of in vitro incubation within the cell culture media. Results indicate that osteoblast cells incubated with composites with low mineral content, fabricated by mineralization of chitosan hydrogel-based scaffolds via the McGrath method, showed enhanced proliferation. Although cells were viable in the presence of composites with high mineral content or nonmineralized chitosan hydrogel-based scaffolds, no significant increase in the cell proliferation rate was observed after 14 days of incubation. Confocal microscope images were not entirely in accordance with these findings. This may be attributed to some of the cells being washed-off from the scaffold surface during sample preparation due to weaker cell-scaffold interactions. This may be due to the staining protocol not being standardized for the chitosan hydrogel-based scaffolds and the possible variability in the nanotopography of the scaffold surface interacting with the cells. Nonetheless, it was observed that chitosan 
hydrogel-based scaffolds facilitate osteoblast adhesion and proliferation in the presence and absence of calcium carbonate.

Data indicates that chitosan hydrogel-based scaffolds and composites are viable materials to be used for bone tissue engineering applications. This along with the results obtained from mechanical tests indicate that chitosan hydrogel-based scaffolds with (a) a low mineral content in the form of laterally growing aggregates of nanoparticles $\left(7-14 \% \mathrm{CaCO}_{3}\right)$ intricately associated with the organic matrix obtained as a result of the matrix-induced mineralization method followed in this research, the McGrath method; and (b) a minimal porosity of $44 \%$ (refer to Chapter 3 ) is the most appropriate material developed in this research to be considered for bone graft applications. 


\subsection{Bibliography}

1. Florencio-Silva, R., G.R.d.S. Sasso, E. Sasso-Cerri, M.J. Simões, and P.S. Cerri, Biology of bone tissue: structure, function, and factors that influence bone cells. BioMed Research International, 2015. 2015.

2. Mercado-Pagán, Á.E., A.M. Stahl, Y. Shanjani, and Y. Yang, Vascularization in bone tissue engineering constructs. Annals of Biomedical Engineering, 2015. 43(3): p. 718729.

3. Roseti, L., V. Parisi, M. Petretta, C. Cavallo, G. Desando, I. Bartolotti, and B. Grigolo, Scaffolds for Bone Tissue Engineering: State of the art and new perspectives. Materials Science and Engineering: C, 2017. 78: p. 1246-1262.

4. Bose, S., M. Roy, and A. Bandyopadhyay, Recent advances in bone tissue engineering scaffolds. Trends in Biotechnology, 2012. 30(10): p. 546-554.

5. Stevens, M.M., Biomaterials for bone tissue engineering. Materials Today, 2008. 11(5): p. 18-25.

6. Karageorgiou, V. and D. Kaplan, Porosity of 3D biomaterial scaffolds and osteogenesis. Biomaterials, 2005. 26(27): p. 5474-5491.

7. Cukierman, E., R. Pankov, D.R. Stevens, and K.M. Yamada, Taking cell-matrix adhesions to the third dimension. Science, 2001. 294(5547): p. 1708-1712.

8. Tamaddon, M., S. Samizadeh, L. Wang, G. Blunn, and C. Liu, Intrinsic Osteoinductivity of Porous Titanium Scaffold for Bone Tissue Engineering. International Journal of Biomaterials, 2017. 2017.

9. Chen, Y., J.E. Frith, A. Dehghan-Manshadi, H. Attar, D. Kent, N.D.M. Soro, M.J. Bermingham, and M.S. Dargusch, Mechanical properties and biocompatibility of porous titanium scaffolds for bone tissue engineering. Journal of the Mechanical Behavior of Biomedical Materials, 2017. 75: p. 169-174.

10. Rezwan, K., Q. Chen, J. Blaker, and A.R. Boccaccini, Biodegradable and bioactive porous polymer/inorganic composite scaffolds for bone tissue engineering. Biomaterials, 2006. 27(18): p. 3413-3431.

11. Khan, Y., F.E.-A. Saadiq, and C.T. Laurencin. In vitro and in vivo evaluation of a novel polymer-ceramic composite scaffold for bone tissue engineering. in Engineering in Medicine and Biology Society, 2006. EMBS'06. 28th Annual International Conference of the IEEE. 2006. IEEE. 
12. Miguel, B.S., R. Kriauciunas, S. Tosatti, M. Ehrbar, C. Ghayor, M. Textor, and F.E. Weber, Enhanced osteoblastic activity and bone regeneration using surface-modified porous bioactive glass scaffolds. Journal of Biomedical Materials Research Part A, 2010. 94(4): p. 1023-1033.

13. Venkatesan, J., I. Bhatnagar, P. Manivasagan, K.-H. Kang, and S.-K. Kim, Alginate composites for bone tissue engineering: a review. International Journal of Biological Macromolecules, 2015. 72: p. 269-281.

14. McDaniel, J.S., M. Pilia, V. Raut, J. Ledford, S.M. Shiels, J.C. Wenke, B. Barnes, and C.R. Rathbone, Alternatives to autograft evaluated in a rabbit segmental bone defect. International Orthopaedics, 2016. 40(1): p. 197-203.

15. Ma, P.X. and J.-W. Choi, Biodegradable polymer scaffolds with well-defined interconnected spherical pore network. Tissue Engineering, 2001. 7(1): p. 23-33.

16. Williams, J.M., A. Adewunmi, R.M. Schek, C.L. Flanagan, P.H. Krebsbach, S.E. Feinberg, S.J. Hollister, and S. Das, Bone tissue engineering using polycaprolactone scaffolds fabricated via selective laser sintering. Biomaterials, 2005. 26(23): p. 48174827.

17. Li, J., B. Baker, X. Mou, N. Ren, J. Qiu, R.I. Boughton, and H. Liu, Biopolymer/calcium phosphate scaffolds for bone tissue engineering. Advanced Healthcare Materials, 2014. 3(4): p. 469-484.

18. Samavedi, S., A.R. Whittington, and A.S. Goldstein, Calcium phosphate ceramics in bone tissue engineering: a review of properties and their influence on cell behavior. Acta Biomaterialia, 2013. 9(9): p. 8037-8045.

19. Pina, S., J.M. Oliveira, and R.L. Reis, Natural-based nanocomposites for bone tissue engineering and regenerative medicine: A review. Advanced Materials, 2015. 27(7): p. 1143-1169.

20. Muzzarelli, R., M. Mattioli-Belmonte, C. Tietz, R. Biagini, G. Ferioli, M. Brunelli, M. Fini, R. Giardino, P. Ilari, and G. Biagini, Stimulatory effect on bone formation exerted by a modified chitosan. Biomaterials, 1994. 15(13): p. 1075-1081.

21. Seol, Y.-J., J.-Y. Lee, Y.-J. Park, Y.-M. Lee, I.-C. Rhyu, S.-J. Lee, S.-B. Han, and C.P. Chung, Chitosan sponges as tissue engineering scaffolds for bone formation. Biotechnology Letters, 2004. 26(13): p. 1037-1041.

22. Prabaharan, M. and P. Sivashankari, Prospects of bioactive chitosan-based scaffolds in tissue engineering and regenerative medicine, in Chitin and Chitosan for Regenerative Medicine. 2016, Springer. p. 41-59. 
23. Levengood, S.K.L. and M. Zhang, Chitosan-based scaffolds for bone tissue engineering. Journal of Materials Chemistry B, 2014. 2(21): p. 3161-3184.

24. Goreham, R.V., A. Mierczynska, L.E. Smith, R. Sedev, and K. Vasilev, Small surface nanotopography encourages fibroblast and osteoblast cell adhesion. RSC Advances, 2013. 3(26): p. 10309-10317.

25. Nguyen, A.T., S.R. Sathe, and E.K. Yim, From nano to micro: topographical scale and its impact on cell adhesion, morphology and contact guidance. Journal of Physics: Condensed Matter, 2016. 28(18): p. 183001. 


\section{Conclusion \& Future Research}

This thesis describes the strategies implemented to develop 3D composites mimicking several of the structural and compositional features of bone and nacre using 3D printing technology and a specialized mineralization technique, the McGrath mineralization method. The applicability of the $3 \mathrm{D}$ porous composites thus generated as a prospective bone graft material was assessed based on their performance when subjected to various forms of compressive mechanical stress, and in an in vitro biological environment.

The following conclusions were deduced from this research:

(a) Chitosan hydrogel can be modified in order to optimize its rheological properties (viscosity and flow behaviour) such that it is suitable for application as the printing ink in custom-designed nozzle extrusion-based 3D printers by varying the $\mathrm{pH}$ of the solution. By manipulating the physical crosslinking of the hydrogel, stable 3D structures with a minimum of $1.5 \mathrm{~mm} \times 1.5 \mathrm{~mm}$ macropores can be fabricated via 3D printing using $5 \% \mathrm{w} / \mathrm{v}$ chitosan hydrogel prepared in the presence of $2 \% \mathrm{v} / \mathrm{v}$ acetic acid as the printing ink in our custom-designed 3D printer equipped with a $0.42 \mathrm{~mm}$ extrusion nozzle. The two central features of the hydrogel flow behaviour are (1) the pressure required to initiate the flow; i.e. the magnitude of the viscosity at low shear rate. As this increases, the pressure required to initiate flow subsequently increases; and (2) the viscosity at the extrusion nozzle. If the viscosity is too high at the shear rate experienced at the extrusion nozzle, extrusion will not occur and if it is too low, the print form will not be maintained.

(b) Cryo-scanning electron microscopy analysis showed that the as-fabricated wet 3D printed scaffolds are hierarchically porous with pores ranging from nano (inherent pores in the hydrogel) to macroscale (printed pores). SEM analysis showed that freezedrying and critical point-drying of the scaffolds can both be used to retain this hierarchical porosity. However, such scaffolds undergo irreversible deformations under very low compressive strain loads and hence are deemed unsuitable for application as load-bearing bone grafts. 
(c) Air-drying of the wet 3D printed chitosan hydrogel-based scaffolds results in dimensional shrinkage, with concomitant loss of porosity during the drying process due to the surface tension effects of the evaporating solvent. Despite this, this method was deemed to be the most suitable drying technique of the three techniques studied, making them better candidates for use in load-bearing bone graft applications as the resultant materials have a higher mechanical strength and stability. Furthermore, it was found that some of this "lost" porosity can be regained when the air-dried scaffolds are rehydrated in physiologically relevant ( $\mathrm{pH}$ 7.2) solvents, thus indicating the presence of an interconnected open pore network in the hydrated chitosan hydrogel.

(d) The McGrath mineralization method can be used to mineralize 3D printed chitosan hydrogel-based scaffolds to develop stable 3D composites while retaining the original 3D printed structure (in the wet state). However, in order to effectively replicate the nacre-like structure that was obtained previously in 2D chitosan hydrogel-based films, in the 3D systems, the protocol followed for McGrath mineralization required modification. In the modified method, the mineralization is performed when the scaffolds are in their wet state such that the extensive porous structure of the chitosan hydrogel facilitates diffusion of the mineralization solution throughout the 3D structure, thus increasing the extent of $\mathrm{CaCO}_{3}$ mineralization occurring within the scaffold.

(e) Introduction of polyacrylic acid (PAA) as the crystal growth modifier, incorporated in each step of the modified mineralization method, results in a changed crystal morphology. The formation of a polyelectrolyte complex between PAA and the chitosan matrix facilitates the hierarchical growth of calcium carbonate crystallites in association with the polymer matrix throughout the 3D structure. The resultant crystals predominantly exist as aggregates of nanosized calcium carbonate crystallites growing laterally in association with the chitosan hydrogel matrix. PAA-induced McGrath mineralization facilitates enhanced particle-matrix interactions that result in the formation of crystallites that are entangled with the polymer matrix via electrostatic interaction. This, in turn, results in stronger association of the minerals to the organic matrix.

(f) The morphology and size of the resultant calcium carbonate crystallites formed within the composites can be varied by changing (1) the concentration of the PAA; (2) the concentration of the mineral precursor ion solutions used; and (3) the period of exposure to the mineralization media. By modulating these parameters, the mineralization strategy results in the development of pancake-like crystallites, imitating early stage 
mineralization in nacre, throughout the hydrogel-based 3D scaffold. Accordingly, it was found that chitosan hydrogel-based scaffolds mineralized via the McGrath method by alternately soaking in $0.25 \mathrm{M}$ precursor ion solution for $1 \mathrm{~h}$ each and then soaking in Kitano solution (saturated calcium carbonate solution) for 3 days results in the formation of appreciable amounts of pancake-like $\mathrm{CaCO}_{3}$ crystallites throughout the exposed regions of the scaffold as well as in between the printed layers. Hence, effectively replicating the "brick-mortar" architecture of nacre on a macroscale in 3D printed chitosan hydrogel-based scaffold.

(g) The extent of mineralization that can be achieved within 3D chitosan hydrogel-based scaffolds when mineralized via the McGrath method is increased by (1) introducing macropores into the printed structure; (2) increasing the period of exposure to the mineralization media; and (3) increasing the concentration of the precursor ion solutions used. However, increasing the extent of mineralization that can be achieved with the $3 \mathrm{D}$ scaffolds results in modification of the morphology of the resultant crystallites, from laterally growing aggregates of nanoparticles in the form of pancakelike structures to large micron-sized globular structures growing on top of pancake-like structures. Hence, there is a trade-off between the extent of mineralization that can be achieved with 3D chitosan hydrogel-based scaffolds and optimization of the morphology of the resultant crystallites in the final composite.

It is important to note that when using diffusion-driven solution-based mineralization processes that proceed via heterogenous nucleation of minerals on a polymer matrix from a solution, such as the McGrath method, that after the initial nucleation and growth of the minerals, which occurs in intimate association with the organic matrix, any further mineral formation/deposition occurs mostly without the active involvement of the polymer matrix. This is to say that, when trying to increase the extent of mineralization occurring throughout the scaffold in a static scaffold environment, as was the case here, by, for example, increasing the concentration of the mineralization media, the extent of interaction between the minerals formed and the polymer matrix is compromised as the mineral formation/deposition increases. This results in some crystals not being in strong association with the polymer scaffold and they are therefore prone to being lost during any subsequent processing steps. This currently remains an issue for this fabrication method. 
Studies investigating the applicability of a simpler and less time-consuming fabrication technique whereby blended chitosan- $\mathrm{CaCO}_{3}$ hydrogels are used as printing inks (i.e. a single-step method) to develop 3D composites with properties similar to those developed via McGrath mineralization of 3D printed chitosan hydrogel-based scaffolds (two-step method) showed that the former has many drawbacks. Most importantly, the loss of inorganic content from the printed structure during the printing process; the limited control over tuning the morphology of the resultant $\mathrm{CaCO}_{3}$ crystallites to form, for example, the desired pancake-like structure; and finally, the lack of good interaction between the organic and the inorganic components within the final composite. As these can detrimentally affect the mechanical properties of the final composite structure, this technique was deemed ineffective for fabricating chitosan calcium-carbonate composites with the desired properties. However, it was found that this one-step method enhanced the thermal stability of the final composites as was the case for the composites developed using the two-step method.

On this basis, various tests to evaluate the behaviour of the chitosan hydrogel-based scaffolds and composites, fabricated only by the two-step method, under compressive stress were performed. Data obtained from uniaxial compression in static mode showed that:

(1) yield ultimate strength and the compressive modulus of air-dried chitosan hydrogelbased scaffolds did not vary significantly when the porosity of the scaffolds was varied from $51.6 \pm 1.3 \%$ to $58.8 \pm 1.2 \%$, achieved by increasing the printed macropore dimensions from $0.62 \pm 0.01 \mathrm{~mm}$ to $0.99 \pm 0.13 \mathrm{~mm}$;

(2) the mechanical properties of chitosan hydrogel-based scaffolds can be increased via mineralization. However, a high mineral content $(>40 \%)$ was shown to affect the strength detrimentally;

(3) the size of the crystallites contributes to the mechanical properties of the resultant composite wherein, composites with large micron-sized particles have lower mechanical strength; and

(4) although the morphology of the mineral crystallites within a composite affects its mechanical properties, it was inconclusive at this stage whether $\mathrm{CaCO}_{3}$ crystallites in the form of pancake-like structures is advantageous or not in improving the mechanical properties of chitosan hydrogel-based composites.

Dynamic compression testing allows further evaluation of the performance of the composites under physiologically relevant conditions. Dynamic mechanical analysis 
revealed that the storage and complex moduli of chitosan hydrogel-based scaffolds and composites:

(1) do not vary when the stress loading frequency is low $(1-9 \mathrm{~Hz})$;

(2) increases for mid-range frequencies, $9-13 \mathrm{~Hz}$, irrespective of the mineral content in the sample; and

(3) is constant beyond $13 \mathrm{~Hz}$, wherein composites have higher modulus compared to nonmineralized scaffolds.

The loss modulus, on the other hand, is effectively constant irrespective of the loading frequency or the mineral content. Analysis of DMA-tested samples showed that dynamic stress loading at high frequencies can cause irreversible deformation of the 3D printed chitosan hydrogel-based scaffolds irrespective of the mineral content. Nonetheless, dynamic loading, within 1-15 Hz loading frequency, does not result in complete fracture of the 3D structures, showing that these structures are capable of effectively dissipating stress when subjected to physiologically relevant compressive stress.

While mechanical properties are critically important in the context of developing bone grafts, equally important is the interaction of the materials with living cells. Thus, a series of relevant biological testing was undertaken. In vitro osteoblast cell culture in the presence of chitosan hydrogel-based scaffolds and composites revealed that these structures are biologically active, non-toxic and facilitate cell adhesion, growth and proliferation. Cell proliferation analysis showed that the number of cells significantly increased within 14 days of incubation with composites with very low mineral content, i.e., those fabricated by mineralization of chitosan hydrogel-based scaffolds via the McGrath method in the presence of $0.1 \mathrm{M}$ precursor ion solutions ( $24 \mathrm{~h}$ alternate soaking +1 week Kitano) in comparison to incubation with non-mineralized scaffold or composites with very high mineral content. Confocal microscope images revealed that chitosan hydrogel-based composites support osteoblast cell adhesion.

Overall, it is concluded that hierarchically porous chitosan-calcium carbonate composite structures fabricated by mineralization of 3D printed chitosan hydrogel-based scaffolds via the McGrath method in the presence of polyacrylic acid, can be considered as materials that achieve many of the structural, mechanical and biological requirements of an ideal bone graft. Hence, this work paves the way for further research whereby various components need to be optimized to enhance their viability. 
Herein it has been shown that mechanically strong and biologically active biopolymermineral composites can be fabricated by the combination of hydrogel-based 3D printing technology and relatively simple mineralization processes and drying techniques. This is a significant advancement over other reported materials. Using 3D printing to generate material based on physically crosslinked chitosan polymer chains has been reported previously [1, 2]. In these studies, however, lyophilization was employed in order to preserve the architecture and the porous (inherent porosity and the printed pores) structure of the scaffolds thus generated. Here it has been shown that an air-drying process can be used instead of lyophilization in order to dry chitosan hydrogel-based scaffolds, maintaining biologically relevant porosity that can be regained upon rehydration in physiological solutions, while also ensuring that complete dissolution of the scaffold does not occur. It is noted that, while the mechanical strength of the scaffolds is altered in different hydrated states, the fully air-dried samples have mechanical response profiles that compare in form and magnitude with bone and that these are superior to other materials reported in the literature. Hence, these materials can be used as a basis for preparing partially hydrated materials with favourable mechanical profiles.

Although this research is not the first in the area of 3D printing using chitosan hydrogels or developing composites through biomimetic mineralization techniques [3-8], it is to the best of our knowledge the first to combine the two with an objective of developing artificial bone grafts. The work has shown that the specialized mineralization techniques developed herein can be effectively modulated to develop bioactive composites wherein the organic component (chitosan) is intricately associated with the mineral component (calcium carbonate), thus imparting a mechanical strength much higher than those reported previously and within the range of trabecular bone (in dry state) [6, 9-11]. Hence, the research summarized in this thesis provides a good foundation for further research in the direction of developing 3D porous bone grafts based on biopolymer hydrogels and ceramics.

\section{Future research:}

Now that it has been established that $3 \mathrm{D}$ chitosan- $\mathrm{CaCO}_{3}$ composites developed via the mineralization of chitosan hydrogel-based scaffolds via the McGrath method in the presence of polyacrylic acid and with architectural features based on the CAD model chosen in this research, exhibit considerable mechanical strength and biological relevance to be considered as a prospective bone graft material, the following future research is suggested: 
- Strategies to automate the printing process in order to facilitate the fabrication of larger structures based on the same CAD model using chitosan hydrogel as the printing ink in a less time-consuming manner needs to be devised. Hence, facilitating the fabrication of scaffolds that comply with the dimensional specifications prescribed in ASTM compression testing protocol for biomaterials. Most of the currently reported methods applicable for this purpose involve chemical crosslinking processes that will hamper the inherent porosity and chemistry of the resultant chitosan hydrogel-based scaffold and hence affect the mineralization kinetics when using the McGrath mineralization method. Thus, the automation process must ensure that the 3D printed scaffolds thus developed are sufficiently porous with open interconnected pores to ensure extensive mineralization of the $3 \mathrm{D}$ scaffolds and that the chemistry of the biopolymer hydrogel is maintained such that the mineralization kinetics are not affected.

- The mechanical properties of the chitosan hydrogel-based scaffolds and composites in a physiologically relevant rehydrated state need to be investigated. The mechanical properties of the chitosan-calcium carbonate composites in their dried state were investigated and found to be favourable, however, it was observed (in Chapter 6) that these composites swell in the presence of culture media (suitable for osteoblast cell proliferation), although this was not the case in PBS, thus changing their viscoelastic properties. On this basis, it is therefore important to characterize these structures in the hydrated state to gain a better understanding of the mechanical performance of the composites in more biologically relevant environments and states.

- Finally, extensive in vitro analysis of the osteoblast differentiation, bone matrix formation and biodegradation kinetics of the scaffold is recommended in order to further evaluate the biological properties of low mineral chitosan hydrogel-based composites. 


\subsection{Bibliography:}

1. Ang, T., F. Sultana, D. Hutmacher, Y.S. Wong, J. Fuh, X. Mo, H.T. Loh, E. Burdet, and S.-H. Teoh, Fabrication of 3D chitosan-hydroxyapatite scaffolds using a robotic dispensing system. Materials Science and Engineering: C, 2002. 20(1): p. 35-42.

2. Murphy, W., J. Black, and G.W. Hastings, Handbook of biomaterial properties. 2016: Springer.

3. Thein-Han, W. and R. Misra, Biomimetic chitosan-nanohydroxyapatite composite scaffolds for bone tissue engineering. Acta Biomaterialia, 2009. 5(4): p. 1182-1197.

4. Zhang, Y. and M. Zhang, Cell growth and function on calcium phosphate reinforced chitosan scaffolds. Journal of Materials Science: Materials in Medicine, 2004. 15(3): p. 255-260.

5. $\mathrm{Hu}$, Q., B. Li, M. Wang, and J. Shen, Preparation and characterization of biodegradable chitosan/hydroxyapatite nanocomposite rods via in situ hybridization: a potential material as internal fixation of bone fracture. Biomaterials, 2004. 25(5): p. 779-785.

6. Levengood, S.K.L. and M. Zhang, Chitosan-based scaffolds for bone tissue engineering. Journal of Materials Chemistry B, 2014. 2(21): p. 3161-3184.

7. Manjubala, I., S. Scheler, J. Bössert, and K.D. Jandt, Mineralisation of chitosan scaffolds with nano-apatite formation by double diffusion technique. Acta Biomaterialia, 2006. 2(1): p. 75-84.

8. Chesnutt, B.M., A.M. Viano, Y. Yuan, Y. Yang, T. Guda, M.R. Appleford, J.L. Ong, W.O. Haggard, and J.D. Bumgardner, Design and characterization of a novel chitosan/nanocrystalline calcium phosphate composite scaffold for bone regeneration. Journal of biomedical materials research Part A, 2009. 88(2): p. 491-502.

9. Hvid, I., P. Christensen, J. Søndergaard, P.B. Christensen, and C.G. Larsen, Compressive Strength of Tibial Cancellous Bone: Instron ${ }^{\circledR}$ and Osteopenetrometer Measurements in an Autopsy Material. Acta Orthopaedica Scandinavica, 1983. 54(6): p. 819-825.

10. Kopperdahl, D.L. and T.M. Keaveny, Yield strain behavior of trabecular bone. Journal of Biomechanics, 1998. 31(7): p. 601-608.

11. Goulet, R.W., S.A. Goldstein, M.J. Ciarelli, J.L. Kuhn, M. Brown, and L. Feldkamp, The relationship between the structural and orthogonal compressive properties of trabecular bone. Journal of Biomechanics, 1994. 27(4): p. 375379-377389. 


\section{Appendix 1}

\section{Uniaxial compression testing of 3D printed ABS-based structures}

Data obtained from uniaxial unconfined compression tests performed on 3D printed acrylonitrile butadiene styrene (ABS) thermoplastic plastic-based structures with different internal architectures (images shown) are shown here.

The dimensions of all the structures tested are $20 \mathrm{~mm}$ (diameter) $\times 40 \mathrm{~mm}$ (height), the thickness of individual struts forming the 3D structure is $0.9 \mathrm{~mm}$. All CAD models are designed by using a basic structure in which the first layer contains struts placed $1.5 \mathrm{~mm}$ apart from each other. In the case of a cubic lattice-based structure this results in the formation of macropores $1.5 \mathrm{~mm} \times 1.5 \mathrm{~mm}$ in dimension when the overlying printed layers are rotated by $0-90-0^{\circ}$. To obtain hexagonal lattices, individual printed layers are rotated by $0-60-120^{\circ}$.

For the hollow cylinder + lattice-based structures, the first layer is a combination of a hollow cylinder enclosing struts placed $1.5 \mathrm{~mm}$ apart. The same rotation patterns are followed for the overlying printed layers as described above to obtain cubic or hexagonal lattices respectively. One sample of each type was tested.

Compression testing was performed in accordance with ASTM D695-10 testing standards for rigid plastics using Instron 1126 compression testing machine at a crosshead speed of $1 \mathrm{~mm} / \mathrm{s}$. The structures were compressed until failure and the compressive strength was calculated. The compressive strength, which is a property that is dependent on both the architecture and the material properties of a given structure, was estimated based on the stress (nominal) carried by the specimen when buckling or rupture of the sample begins. The yield stress was calculated based on the point of deviation from the linear-elastic region in the stress-strain graph as explained in Chapter 5. 

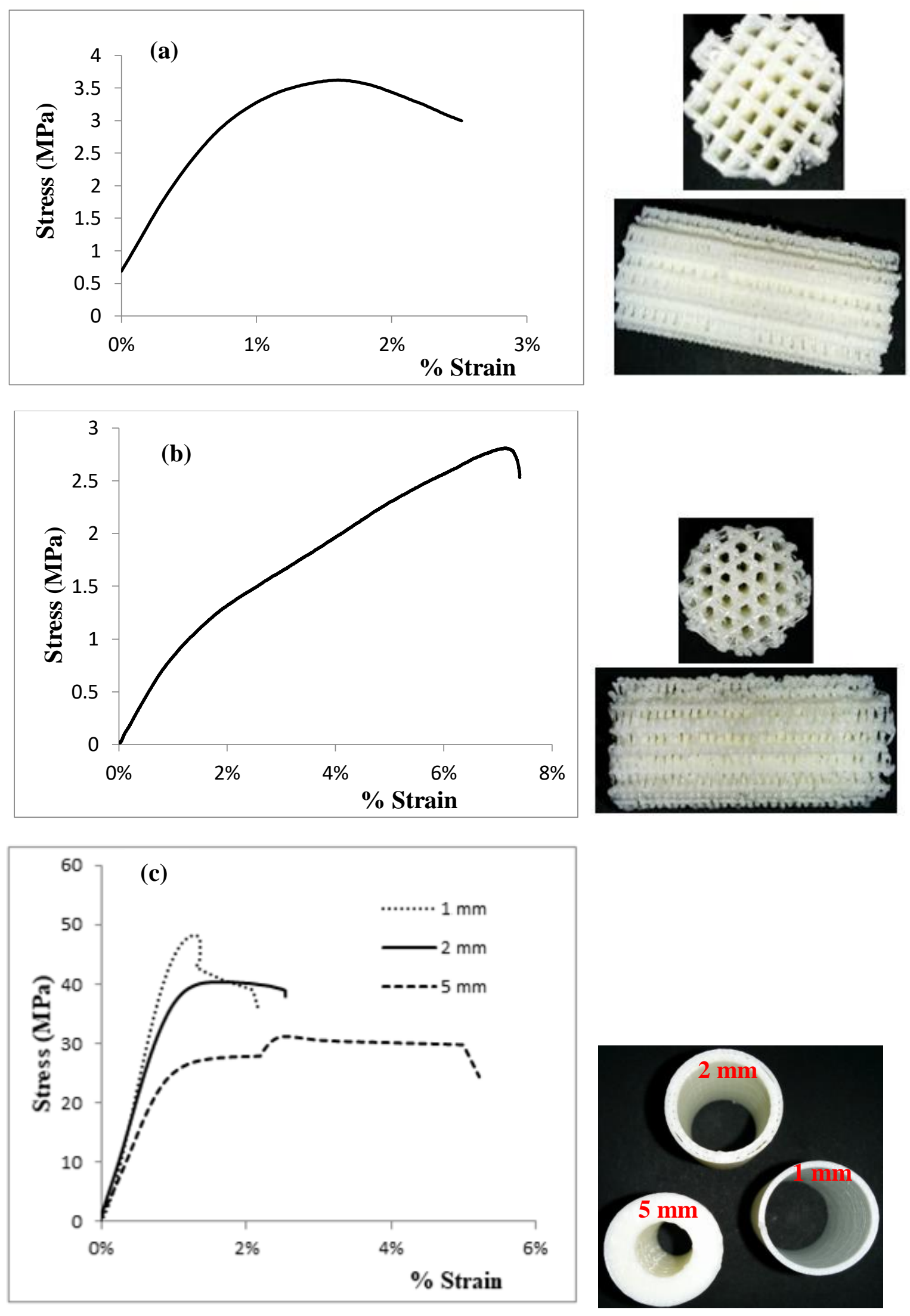

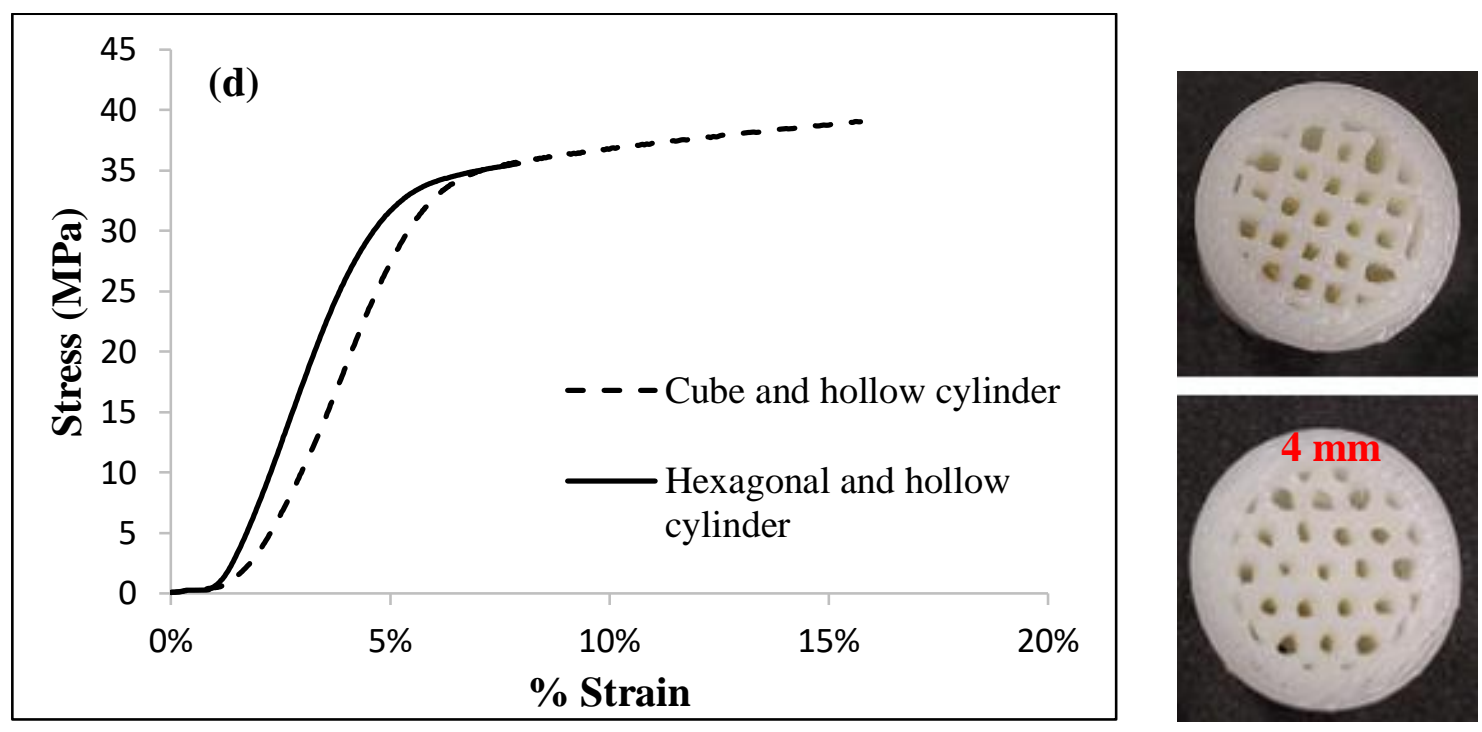

Figure A1.1: Stress-strain plots for 3D printed ABS-based structures based on (a) cubic lattices; (b) hexagonal lattice; (c) hollow cylinders with varying wall thickness; and (d) combination of hollow cylinder (4 $\mathrm{mm}$ wall thickness) and cubic or hexagonal lattices.

\begin{tabular}{|c|c|c|}
\hline Geometry of the test specimens & Yield stress (MPa) & $\begin{array}{c}\text { Compressive } \\
\text { strength (MPa) }\end{array}$ \\
\hline Cylinder based on cubic lattice & $2.44 \pm 0.01$ & $3.00 \pm 0.01$ \\
\hline Cylinder based on hexagonal lattice & $0.76 \pm 0.01$ & $2.73 \pm 0.01$ \\
\hline Hollow cylinder with 1 mm wall thickness & $35.22 \pm 0.02$ & $46.92 \pm 0.02$ \\
\hline Hollow cylinder with 2 mm wall thickness & $30.70 \pm 0.01$ & $40.51 \pm 0.01$ \\
\hline Hollow cylinder with 5 mm wall thickness & $22.31 \pm 0.01$ & $31.20 \pm 0.02$ \\
\hline Cubic lattice surrounded by hollow cylinder & $34.51 \pm 0.01$ & $38.91 \pm 0.02$ \\
\hline Hexagonal lattice surrounded by hollow & $31.42 \pm 0.01$ & $34.28 \pm 0.01$ \\
\hline cylinder & & \\
\hline
\end{tabular}

Table A1.1: The variation in the yield stress and compressive strength of $3 D$ printed $A B S$ based structures with different architectures. Only one sample of each type was used. 
